This dissertation has been

microfilmed exactly as received $\quad 69-15,978$

YU, Jiunn-Shang, 1936-

MEASUREMENT OF CONSTITUTIVE PARAMETERS

USING THE MIE SOLUTION OF A SCATTERING

SPHERE.

The Ohio State University, Ph.D., 1969

Engineering, electrical

University Microfilms, Inc., Ann Arbor, Michigan 


\section{MEASUREMENT OF CONSTITUTIVE PARAMETERS USING THE MIE SOLUTION OF A SCATTERING SPHERE}

\section{DISSERTATION}

Presented in Partial Fulfillment of the Requirements

for the Degree Doctor of Philosophy in the

Graduate School of The Ohio State University

\section{By}

Jiunn-Shang Yu, B.S.E.E., M.Sc.

$$
\text { ****** * }
$$

The Ohio State University

1968

Approved by

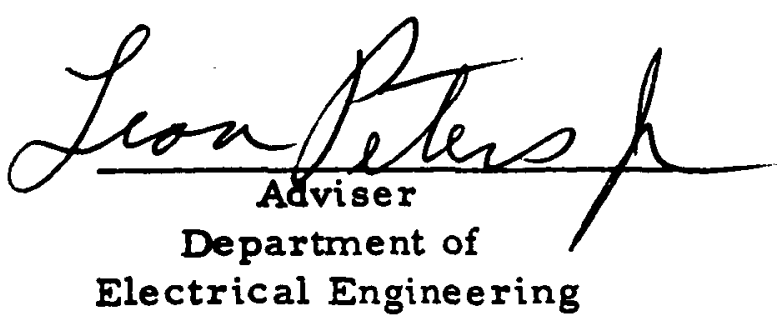




\section{ACKNOWLEDGMENTS}

The work reported in this study was supported in part by Contract F33615-67-C-1507 between Air Force Avionics Laboratory, Air Force Systems Command, Wright-Patters on Air Force Base, Ohio and The Ohio State University Research Foundation.

The author wishes to thank Professor Leon Peters, Jr. for guidance and encouragement, Professor William H. Peake for helpful discussions, and Professor Roger C. Rudduck for critical suggestions in completion of this study.

The author is indebted to Dr. David L. Moffatt who initially proposed the use of quasistatic solution from which this study is derived, and Professor Edward M. Kennaugh who suggested the technique of target rotation in experimentations.

Construction of the refractive index chart was originally helped by Mr. Chris Spentzos, later programmed and plotted by Mr. Dale Castello, and finally edited and drafted by Mr. Everett Huey. Experimental measurements were made by Messers. Summers A. Redick and Norman L. Vesley. Editorial assistance is from Mr. Raymond C. Dosky and manuscript preparation is by Mrs. Eleanor E. Traxler. Their patient efforts are greatly appreciated. 


\section{VITA}

7 September 1936

$1955-1958 \ldots .$.

$1959 \ldots . .$.

$1960-1961 \ldots$

$1961-1962 \ldots .$.

$1965-1968$
Born - Taichung, Taiwan, China

Diploma, Taipei Institute of Technology

Chinese Air Force Electronics and Communication School

B.S.E.E., The University of Florida, Gainesville, Florida

M.Sc., The University of Florida, Gainesville, Florida

The Ohio State University, Columbus, Ohio

\section{PUBLICATIONS}

"On Electron Streams of Ionized Gases," (M.Sc. Thesis, University of Florida, June 1962)

"Comprehensive Analysis for E-Plane of Horn Antennas by Edge Diffraction Theory." IEEE Trans. on Antennas and Propagation, Vol. AP-14, No. 2, March 1966

"On Higher-Order Diffracti on Concepts Applied to a Conducting Strip." IEEE Trans. on Antennas and Propagation, Vol. AP-15, No. 5, September 1967

"On Description and Normalization of Energies or Powers of Electric Circuits." International Conference on Microwave Circuit Theory and Iriormation Theory, Tokyo, Japan, September 1964

"Analysis of Radiation Characteristics by Diffraction Theory in the Principal Plane of Typical Antennas." IEEE International Antenna and Propagation Symposium, Washington, D.C., September 1965 


\section{VITA (Cont.)}

"A New Form of Diffraction Coefficients and Its Application to a Thick Conducting Wall." URSI Spring Meeting, Washington, D.C., April 1966

"On Higher-Order Diffraction Concepts Applied to a Conducting Strip." IEEE International Antenna and Propagation Symposium, Palo Alto, California, December 1966

"Determination of the Constitutive Parameters by Scattered Fields of Spherical Bodies." IEEE International Antenna and Propagation Symposium, Palo Alto, California, December 1966

\section{FIELDS OF STUDY}

Major Fields: Electrical Engineering

Studies in Electromagnetic Theory. Professors Robert G. Kouyoumjian and Leon Peters, Jr.

Studies in Antenna Theory. Professor Roger C. Rudduck

Studies in Network Synthesis. Professors Wayne H. Chen and William C. Davis

Studies in Classical Mechanics. Professor Wave H. Shaffer

Studies in Applied Mathemetics. Professors Henry D. Colson and Stefan Drobot 


\section{ILLUSTRATIONS}

Figure

Page

1 Coordinates systems of a sphere of diameter d illuminated by a honogeneous plane wave

2 The functions $\mathrm{p}_{1}$ to $\mathrm{p}_{4}$ of bistatic angle $\theta$

3 The functions $q_{1}$ to $q_{4}$ of bistatic angle $\theta$

4 Some principal contours of $X_{1}\left(v_{1} \rho_{O}\right)$ in the $v_{\text {rpo-plane. }}$

5 Six repetitive regions and four saddle point regions of $\mathrm{X}_{1}\left(v_{\mathrm{r}} \rho_{\mathrm{O}}\right)$ in the $v_{\mathrm{r}} \rho_{\mathrm{O}}$-plane

6 The $X_{1}$-plane representation of those regions in Fig. 5

7 A $1 \%$-circle centered at $S_{1}$ in the first saddle point region of $X_{1}$-plane.

8 The 18\%-circle centered at $C_{1}$ of $v_{x} P_{0}-p l a n e$ corresponding to the $\mathrm{X}_{1}$-plane of Fig. 7

9 Outline of the figures which give in Appendix II the detailed contour plots of the refractivi index chart

10 A portion of the chart used for numerical illustrations

11 An uncertainty circle of $a \gamma$ and its four representative data points separated by $0.05 \mathrm{~dB}$ and $0.5^{\circ}$

12 Complex-plane representation of the data in Table II 


\section{ILLUSTRATIONS (Cont.)}

Figure

Page

13 Distribution of $\eta_{x}$ from consistent solutions produced by use of the data in row 3 of Table I

14 Distribution of $v_{I} P_{0}$ from consistent solutions produced by use of the data in row 3 of Table I

15 Distribution of $\eta_{r}$ from consistent solutions produced by use of the data in row 1 of Table I

16 Distribution of $\nu_{r} P_{0}$ corresponding to Fig. 15

17 Distribution of $\eta_{r}$ from consistent solutions by us the the data in row 2 of Table I

Distribution of $\nu_{r} P_{0}$ corresponding to Fig 17

19 Distributions of dipole coefficients $\left(a_{1}, b_{1}\right)$ obtained from the consistent process

20 Distributions of quadrupole coefficients $\left(a_{2}, b_{2}\right)$ obtained from the consistent process

21 Geometry for the E-plane measurements at 6 and $7 \mathrm{GHz}$

22 Geometry for the H-plane measurements at 6 and $7 \mathrm{GHz}$

23 Aperture dimensions of the two receiving hog horns

A side view of the dented styrofoam plate used to support the target sphere (the plate can be rotated horizontally $360^{\circ}$ ).

25 The $\mathrm{H}$-plane measurements at $\theta=120^{\circ}$ made for specimens $A$ and $L$ at $7.03 \mathrm{GHz}$ 


\section{ILLUSTRATIONS (Cont.)}

Figure

Page

26 Maximum possible amplitude and phase errors in the E-plane of a conducting sphere of $\rho_{0}=\pi d / \lambda_{0}=0.9$, when $\rho_{0}$ is allowed to change $\pm 0.5 \%$ and $\theta$ is allowed to deviate $\pm 0.5^{\circ}$

27 Maximum possible amplitude and phase errors in the $\mathrm{H}$-plane of a conducting sphere of $\rho_{0}=\pi d / \lambda_{0}=0.9$, when $\rho_{0}$ is allowed to change $\pm 0.5 \%$ and $\theta$ is allowed to deviate $\pm 0.5^{\circ}$

28 Distribution of $\eta_{r}$ from the consistency process applied to specimen $A$ measured at $6.17 \mathrm{GHz}$

29 Distribution of $\eta_{r}$ from the consistency process applied to specimen I measured at $6.17 \mathrm{GHz}$

30 Distribution of $\eta_{r}$ from the consistency process applied to specimen $\mathrm{K}$ measured at $6.17 \mathrm{GHz}$

Distribution of $\eta_{r}$ from the consistency process applied to the specimen $\mathrm{L}$ measured at $6.17 \mathrm{GHz}$

32 Distributions of $X_{1}$ from the consistency process applied to specimens $K$ and $L$ measured at $6.17 \mathrm{GHz}$

33 Distribution of $\nu_{r} P_{0}$ from the consistency process applied to specimen $\mathbb{K}$ measured at $6.17 \mathrm{GHz}$

34 Distribution of $v_{r} f_{0}$ from the consistency process applied to specimen $\mathrm{L}$ measured at $6.17 \mathrm{GHz}$.

35 Normalized bistatic amplitude patterns of specimen $A$ at $6.17 \mathrm{GHz}$ (computed by use of the data in Table VI).

36 Normalized bistatic phase patterns of specimen $A$ at $6.17 \mathrm{GHz}$ (computed by use of the data in Table VI) 


\section{ILLUSTRATIONS (Cont.)}

Figure

Page

37 Normalized bistatic amplitude patterns of specimen I at $6.17 \mathrm{GHz}$ (computed by use of the data in Table VI)

38 Normalized bistatic phase patterns of specimen I at $6.17 \mathrm{GHz}$ (computed by use of the data in Table VI)

39 Normalized bistatic amplitude patterns of specimen $\mathrm{K}$ at $6.17 \mathrm{GHz}$ (computed by use of the data in Table VI)

40 Normalized bistatic phase patterns of specimen $\mathrm{K}$ at $6.17 \mathrm{GHz}$ (computed by use of the data in Table VI)

41 Normalized bistatic amplitude patterns of specimen $\mathrm{L}$ at $6.17 \mathrm{GHz}$ (computed by use of the data in Table VI)

42 Normalized bistatic phase patterns of specimen $\mathrm{L}$ at $6.17 \mathrm{GHz}$ (computed by use of the data in Table VI)

43 Normalized bistatic amplitude pattern of specimen I at $7.03 \mathrm{GHz}$ (computed by use of the nonconsistent results in Table VI)

44 Normalized bistatic phase pattern of specimen I at $7.03 \mathrm{GHz}$ (computed by use of the nonconsistent results in Table VI)

45 Three bistatic scattering patterns, produced by the three sets of $\left(\mu_{r}, \epsilon_{r}\right)$ in Table VII, differ among themselves by less than $0.1 \mathrm{~dB}$ and $1^{\bullet}$

46 Amplitude patterns of the three specimens, characterized by the same parameters $\left(\mu_{r}, \epsilon_{r}\right)$ used for Fig. 45, when their size is. reduced to $p_{0}=0.5$ 


\section{ILLUSTRATIONS (Cont.)}

Figure

Page

47 Two representative values on the 5\%-circle of the parameter $\mu_{r}$

48 Phase error patterns due to 5\%-changes in $\mu_{I}$ of specimen 1 in Table VIII

49 Amplitude error patterns due to 5\%-changes in $\mu_{r}$ of specimen 1 in Table VIII

50 Phase error patterns due to 5\%-changes in er of specimen 1 in Table VIII

51 Amplitude error patterns due to 5\%-changes in er of specimen 1 in Table VIII

52 Phase error patterns due to 5\%-changes in $\mu_{r}$ of specimen 2 in Table VIII

53 Amplitude error patterns due to 5\%-changes in $\mu_{r}$ of specimen 2 in Table VIII

54 Phase error patterns due to $5 \%$-changes in er of specimen 2 in Table VIII

55 Amplitude error patterns due to 5\%-changes in $x$ of specimen 2 in Table VIII

56 Phase error patterns due to 5\%-changes in $\mu_{r}$ of specimen 3 in Table VIII

57 Amplitude error patterns due to 5\%-changes in $\mu_{r}$ of specimen 3 in Table VIII

58 Phase error patterns due to 5\%-changes in ir of specimen 3 in Table VIII

59 Amplitude error patterns due to 5\%-changes in ex of specimen 3 in Table VIII

The first three pairs of $2^{n}$-pole coefficients $\left(a_{n}^{c}, b_{n}^{c}\right)$ of a conducting sphere 


\section{ILLUSTRATIONS (Cont.)}

Figure

Page

61 The principal-plane amplitude patterns for

three different sizes of a conducting sphere

62 The principal E-plane phase patterns for three different sizes of a conducting sphere

63 The principal H-plane phase patterns

corresponding to those in Fig. 62

64 Forward and backscattering cross sections per square wavelength of a conducting sphere

65 The functions $A_{1}\left(p_{0}\right)$ and $B_{1}\left(p_{0}\right)$ used to define the dipole coefficients $\left(a_{1}, b_{1}\right)$

66 The principal-plane amplitude patterns of a sphere with $\rho_{0}=0.1 \pi$ when its dipole coefficients are simultaneously in resonance

67 The principal-plane amplitude patterns of a sphere with $p_{0}=0.1 \pi$ when only its magnetic dipole is in resonance

68 True amplitude patterns compared with those obtained by using an approximate $X_{1}$

69 True phase patterns compared with those obtained by using approximate $X_{1}$

70 Contours of $X_{1}\left(v_{x} P_{O}\right)$ in the region $0 \leq v_{r} p_{0}$ $\leq 3.8$ and $0 \geq v_{r}{ }^{\prime \prime} \rho_{O} \geq-2.0$

71 Contours of $X_{1}\left(v_{r} P_{O}\right)$ in the region $3.8 \leq v_{r} P_{O}$ $\leq 7.5$ and $0 \geq v_{r} " p_{0}>-2.0$

72 Contours of $\mathrm{X}_{1}\left(v_{\mathrm{r}} \rho_{\mathrm{O}}\right)$ in the region $7.5 \leq v_{\mathrm{r}} \mathrm{P}_{\mathrm{O}}$ $\leq 11.4$ and $0 \geq v_{r}{ }^{\prime \prime} p_{0} \geq-2.0$

73 Contours of $X_{1}\left(v_{r} \rho_{O}\right)$ in the region $11.4 \leq v_{Y}^{\prime} \rho_{O}$ $<15.0$ and $0 \geq v_{r}{ }^{\prime \prime} \rho_{0} \geq-2.0$ 


\section{ILLUSTRATIONS (Cont.)}

Figure

74 Contours of $X_{1}\left(v_{r} \rho_{0}\right)$ in the region $0 \leq v_{r} \rho_{0}$

$\leq 3.8$ and $-2.0 \geq v_{x} \rho_{0} \geq-4.0$

75 Contours of $X_{i}\left(v_{r} \rho_{O}\right)$ in the region $3.8 \leq v_{Y}{ }^{\prime} \rho_{O}$

$\leq 7.5$ and $-2.0 \geq v_{x}{ }^{\prime \prime} \rho_{0} \geq-4.0$

76 Contours of $X_{1}\left(\nu_{r} P_{O}\right)$ in the region $7.5 \leq \nu_{r}^{\prime} \rho_{O}$

$\leq 11.4$ and $-2.0 \geq v_{r}{ }^{\prime \prime} p_{0} \geq-4.0$

77 Contours of $X_{1}\left(v_{r} P_{O}\right)$ in regions $11.4 \leq v_{r} P_{O}$

$\leq 15.0$ and $-2.0 \geq v_{r}{ }^{\prime \prime} \rho_{0} \geq-4.0$ Contours of $\mathrm{X}_{\mathrm{f}}\left(v_{\mathrm{r}} \rho_{\mathrm{o}_{1}}\right)$ in the region $0 \leq v_{\mathrm{r}}{ }^{\prime} \rho_{0}$
$\leq 3.8$ and $-4.0 \geq v_{\mathrm{r}} \rho_{0} \geq-6.0$

79 Contours of $X_{1}\left(v_{r} \rho_{O}\right)$ in the region $3.8 \leq v_{r}^{\prime} \rho_{0}$

$\leq 7.5$ and $-4.0 \geq v_{r}{ }^{\prime \prime} p_{0} \geq-6.0$

80 Contours of $X_{1}\left(v_{Y} P_{O}\right)$ in the region $7.5 \therefore v_{r}^{\prime} P_{0}$ $\leq 11.4$ and $-4.0 \geq v_{x} " \rho_{0} \geq-6.0$

81 Contours of $\mathrm{X}_{1}\left(v_{\mathrm{r}} \rho_{\mathrm{O}}\right)$ in the region $11.4 \leq \nu_{r} \rho_{\mathrm{O}}$ $\leq 15.0$ and $-4.0 \geq v_{r} " \rho_{0} \geq-6.0$

82 Contours of $X_{1}\left(v_{r} P_{O}\right)$ in the region $0 \leq v_{r}{ }^{\prime} \rho_{0}$ $\leq 3.8$ and $-6.0 \geq v_{\mathrm{r}}$ " $\rho_{\mathrm{O}} \geq-8.0$

83 Contours of $X_{1}\left(v_{r} \rho_{O}\right)$ in the region $3.8 \leq v_{x} \rho_{O}$

$\leq 7.5$ and $-6.0 \geq v_{r}$ " $p_{0} \geq-8.0$

84 Contours of $X_{1}\left(v_{r} \rho_{O}\right)$ in the region $7.5 \leq v_{r}^{\prime} \rho_{O}$ $\leq 11.4$ and $-6.0 \geq v_{x}{ }^{\prime \prime} \rho_{0} \geq-8.0$

85 Contours of $\mathrm{X}_{1}\left(\nu_{\mathrm{r}} \rho_{0}\right)$ in the region $11.4 \leq \nu_{\mathrm{r}}^{\prime} \rho_{\mathrm{O}}$ $\leq 15.0$ and $-6.0 \geq v_{x}$ " $\rho_{0} \geq-8.0$ 


\section{LIST OF TABIES}

Table

Page

I THE VALUES OF $y_{\theta}\left(\theta_{i}\right)$ AND $\chi_{\phi}\left(\theta_{i}\right)$ USED TO ILLUSTRATE THE CONSISTENCY PROCESS

II SOLUTIONS ESTIMATED BY USE OF THE DATA IN TABLE I

III EXAMPLES OF DATA REDUCED FROM THE H-PLANE MEASUREMENTS AT $7.03 \mathrm{GHz}$

IV SUMMARIZED STATEMENTS ON MEASUREMENTS AND COMPUTATIONS

$V$ ESTIMATED VALUES OF $\left(\eta_{r}, v_{r}\right)$ OBTAINED BY USE OF THE COMBINATIONS IN TABLE IV IN THE CONSISTENCY PROCESS

VI THE ESTIMATED VALUES OF $\left(\mu_{r}, \epsilon_{r}\right)$ OBTAINED FROM THE ESTIMATED

$\left(\eta_{\mathbf{r}}, v_{\mathbf{r}}\right)$ IN TABLE $V$

VII THREE SETS OF $\left(\mu_{r}, \epsilon_{r}\right)$ USED TO ILLUSTRATE "NONUNIQUENESS" AS SHOWN IN Fig. 45

VIII THREE SETS OF $\mu_{r}$ AND \&r WITH 5\% CHANGES INTRODUCED TO STUDY THE CONSEQUENT CHANGES IN SCATTERED FIELDS

IX THE $2^{n}$-POLE COEFFICIENTS OF A SMALL CONDUCTING SPHERE

$X$ THE $2^{n}$-POLE COEFFICIENTS OF THREE SPHERES OF THE SAME SIZE BUT OF DIFFERENT MATERIALS 
CONTENTS

Page

ACKNOWLEDGEMENTS. ................

vitA. .....................

ILLUSTRATIONS. .................. iv

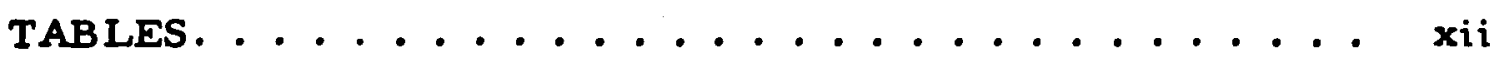

Chapter

I INTRODUCTION. ................. 1

II NORMALIZED BISTATIC FIELDS DEFINED

BY THE MIE SOLUTION. ........... 6

A. General Forms of the Scattered

Fields $\left(E_{\theta}, E_{\phi}\right)$

B. Normalized Bistatic Fields of a Small Specimen Sphere 13

C. Steps in Determination of $\left(\mu_{r}, e_{r}\right)$ from

Measured Scattered Fields 16

III THE REFRACTIVE INDEX CHART. ...... 20

A. A Transcendental Equation with Complex Roots

B. The Chart and the Appropriate Root 27

C. Inaccuracies and Limitations 31

IV THE CONSISTENCY PROCESS. ......... 33

A. The Uncertainty Circle in the $\gamma$-Plane 33

B. Numerical Examples for the Consistency

Process

C. The Consistency Process Summarized 50 
CONTENTS (Cont.)

Chapter

Page

V EXPERIMENTATIONS AND FINAL RESULTS. . . 52

A. Transmitter and Receiver Systems 52

B. Scattering Range and Target Support 54

C. Measurement Procedure 55

D. Measurement Uncertainties 60

E. Homogeneity anc Isotropicity 62

F. Data Reduction 63

G. Evaluation of Parameters 65

H. Assessment of the Parameters 74

VI NONUNIQUENESS, PERTURBATIONS AND RECOMMENDATIONS. ...........

A. Two Specimen Sizes for a Unique

Set of $\left(\mu_{r}, \epsilon_{r}\right)$

B. Perturbations of Scattered Fields by the Parameters 94

C. Recommendations 104

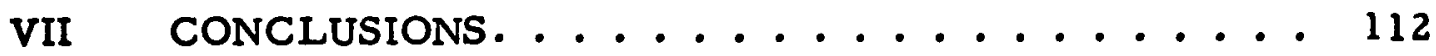

Appendix

I PROPERTIES OF A SMALL SPHERE. . . . . 115

A. Scattering Patterns of a Conducting Sphere 118

B. Resonance Phenomena of $2^{\mathrm{n}}$-pole Coefficients 122

C. Dipoles, Quadrupole, and Octupole Spheres 127

II USEFUL APPLICATIONS AND DETAILED

CONTOURS OF THE REFRACTIVE

INDEX CHART. .............. 130

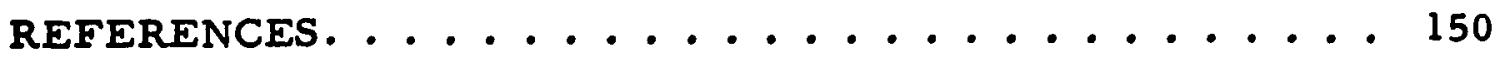




\section{CHAPTER I \\ INTRODUCTION}

Of concern here is a method of determining the constitutive parameters $(\boldsymbol{\mu}, \epsilon)$ which represent the macroscopic properties[ $[1,2,3]$ of a medium. To restrict ourselves to the simplest problem, only passive and stationary media will be considered. Furthermore, we also confine our study to those media which may be assumed homogeneous, isotropic and linear. In free space the value of $\mu$ is adopted by international agreement and the value of $c$ is then determined by the velocity of light. Using subscript 0 to denote the free space parameters, we list

$$
\mu_{0}=4 \pi \times 10^{-7} \text { henry/meter, the free space permeability, }
$$

and

$$
\epsilon_{0} \approx 10^{-9} / 36 \pi \mathrm{farad} / \text { meter, the free space permittivity }
$$

Note that only approximate value is given in Eq. (lb) because the speed of light is usually approximated as $3 \times 10^{8} \mathrm{~meter} / \mathrm{second}$ from the value $299,792.5 \pm 0.4 \mathrm{~km} / \mathrm{sec}$ adopted by URSI in 1957. [4] An alternative set of free space characteristic parameters may be defined 28 
(2a) $\eta_{0}=\sqrt{\mu_{0} / \epsilon_{0}} \approx 120 \pi \mathrm{ohms}$, the free space intrinsic impedance,

and

$$
\begin{array}{r}
k_{0}=\omega \sqrt{\mu_{0} \epsilon_{0}}=2 \pi / \lambda_{0} \text { meter }^{-1} \text {, the free space propagation } \\
\text { constant. }
\end{array}
$$

where $\lambda_{0}$ is free space wavelength and $\omega$ is angular frequency.

It is convenient to characterize a medium by referring its parameters to those of free space. By making use of Eq. (1), a medium of $\mu$ and may be characterized by these relative parameters:

$$
\mu_{r}=\mu / \mu_{0}=\mu_{r}^{\prime}+j \mu_{r}{ }^{\prime \prime} \text {, the relative permeability, }
$$

and

$$
e_{r}=e_{/} \epsilon_{0}=e_{r}^{\prime}+j e_{r}^{\prime \prime} \text {, the relative permittivity. }
$$

Alternatively, by making use of Eq. (2), a medium of $\eta$ and $k$ may be characterized by

$$
\eta_{r}=\eta / \eta_{0}=\eta_{r}^{\prime}+j \eta_{r}^{\prime \prime}=\sqrt{\mu_{r} / C_{r}}, \begin{array}{r}
\text { thelative intrinsic } \\
\text { impedance, }
\end{array}
$$

and

$$
v_{r}=k / k_{0}=v_{r}^{\prime}+j v_{r}^{\prime \prime}=\sqrt{\mu_{r^{e}}} \text {, the refractive index. }
$$

These relative parameters in Eq. (3) and (4) are dimensionless quantities and, as indicated, are in general complex. All the real 
parts of these parameters are positive numbers for passive media. By use of time-convention $\exp (+j \omega t)$, the imaginary parts $\mu r^{\prime \prime}, r^{\prime \prime}$ and $v_{x}^{\prime \prime}$ are required to be negative while $\eta_{r}^{\prime \prime}$ can be either positive or negative for passive media.

A survey on measurement methods of determining $\left(\mu_{r}, \epsilon_{r}\right)$ in different frequency regions has been made by Bussey[5] who listed 65 references up to mid-1966. The present method uses a spherical specimen which is illuminated by a plane wave in free space. Measurements are made to scattered fields in two orthogonal planes of the specimen sphere. The corresponding scattered fields of a conducting sphere are also measured to provide reference amplitudes and phase angles. These measured data are then used in conjunction with the Mie solution[1] to determine the desired parameters $\left(\mu_{Y}, q_{r}\right)$. Since scattered fields in free space are used, our method is considered a new method in microwave frequencies which differs from the previously used perturbation methods $[5,6,7]$ and the methods $[8]$ of transmission and reflection.

For the convenience of explaining the basic procedures involved, we list some pertinent symbols in the following:

$d$ is the diameter of a sphere

$\lambda_{0}$ is the free space wavelength

$P_{0}=\pi d / \lambda_{0}$ is the sphere's circumference in number of $\lambda_{0}$ $(r, \theta, \phi)$ are the spherical coordinates 
$\left(a_{n} ; b_{n}\right)$ are the magnetic and electric $2^{n}$-pole coefficients of a specimen sphere

$\left(a_{n}^{c}, b_{n}^{c}\right)$ are the magnetic and electric $2^{n}$-pole coefficients of a conducting sphere

$\left(E_{\theta} E_{\phi}\right)$ are the $\theta$ - and $\phi$-components of the electric field vector scattered by a specimen sphere

$\left(E_{\theta}^{C}, E_{\phi}^{c}\right)$ are the $\theta$ - and $\phi$-components of the electric field vector scattered by a conducting sphere

$\left(\gamma_{\theta}, \gamma_{\phi}\right)=\left(E_{\theta} / E_{\theta}^{c}, E_{\phi} / E \oint\right)$ are the normalized bistatic fields. The Mie solution[1] expresses the scattered field components $\left(E_{\theta}, E_{\phi}\right)$ or $\left(E_{\theta}^{C}, E_{\phi}^{c}\right)$ as an infinite series summations of the $2^{n}$-pole coefficients $\left(a_{n}, b_{n}\right)$ or $\left(a_{n}^{c}, b_{h}\right)$. When the sphere diameter $d$ is made smaller than $0.5 \lambda_{0}$, the dominant terms in most scattering spheres are the dipole coefficients $\left(a_{1}, b_{1}\right)$ or $\left(a_{1}^{c}, b f\right)$. Experimentally both $\left(E_{\theta}, E_{\phi}\right)$ and $\left(E_{\theta}^{c}, E_{\phi}^{c}\right)$ are measured in two orthogonal planes to obtain the normalized bistatic fields $\left(\gamma_{\theta^{\prime}} \gamma_{\phi}\right)$. These normalized quantities are then used in a system of simultaneous linear equations to solve for $\left(a_{n}, b_{n}\right)$.

It is sufficient to use only the solutions of the dominant coefficients $\left(a_{1}, b_{1}\right)$ to find a set of desired parameters $\left(\mu_{r}, c_{r}\right)$ of a specimen. How ever, it is also possible in theory to find another set of $\left(\mu_{r}, \epsilon_{r}\right)$ from the solutions of $\left(a_{2}, b_{2}\right)$ or from those of higher-order $\left(a_{n}, b_{n}\right)$. Theory requires that these two sets of parameters $\left(\mu_{r}, e_{r}\right)$ should be the same, 
because they are material properties only and this leads to the consistency requirement in the present method. In practice, the consistency requirement can be employed to reduce systematic errors caused by theoretical assumptions and experimental inaccuracies of our scattering method.

The refractive index $v_{r}$ is to be determined from a transcendental function $X_{1}$. The contours of $X_{1}$ plotted in the $\nu_{r} \rho_{0}$ plane is called a refractive index chart and is used to find $v_{x} \rho_{0}$ when $X_{1}$ is known. Some properties and applications of the refractive index chart are to be presented following a summary of the Mie solution. Theoretical examples will then be given to illustrate the inverse process of obtaining the parameters $\left(\eta_{r}, v_{r}\right)$ from a set of specified values $\left(Y_{\theta} Y_{\phi}\right)$. Experimentations and final numerical results for four specimens at 6 and $7 \mathrm{GHz}$ will be described before we finally discuss some problems of nonuniqueness and inaccuracy. 


\section{CHAPTER II \\ NORMALIZED BISTATIC FIELDS DEFINED \\ BY THE MIE SOLUTION}

Normalized bistatic fields are to be defined as ratios of the scattered field components of a specimen sphere to those of a perfectly conducting sphere. Except that we use time-convention exp (t jwt) instead of exp (- iwt), the Mie solution as given in Stratton's Electromagnetic Theory[1] is used here to describe the scattered electric field components $\left(E_{\theta}, E_{\phi}\right)$. A sphere of diameter d surrounded by free space is shown in Fig. 1. The sphere is illuminated by a homogeneous plane wave propagating in the direction of $(+z)$-axis. The two principal planes are the E-plane (xz-plane) in which the incident E-field lies, and the H-plane (yz-plane) in which the incident H-field lies.

\section{A. General Forms of the Scattered Fields $\left(E_{\theta} E_{\phi}\right)$}

A complete solution of the field vectors scattered from a specimen sphere is given as weighted sums of vector functions, as can be found in Eq. (4) in p. 564 of Reference 1. We take from the solution only the electric field components $E_{\theta}$ at $\phi=0$ and $E_{\phi}$ at $\phi=90^{\circ}$. They are 
7

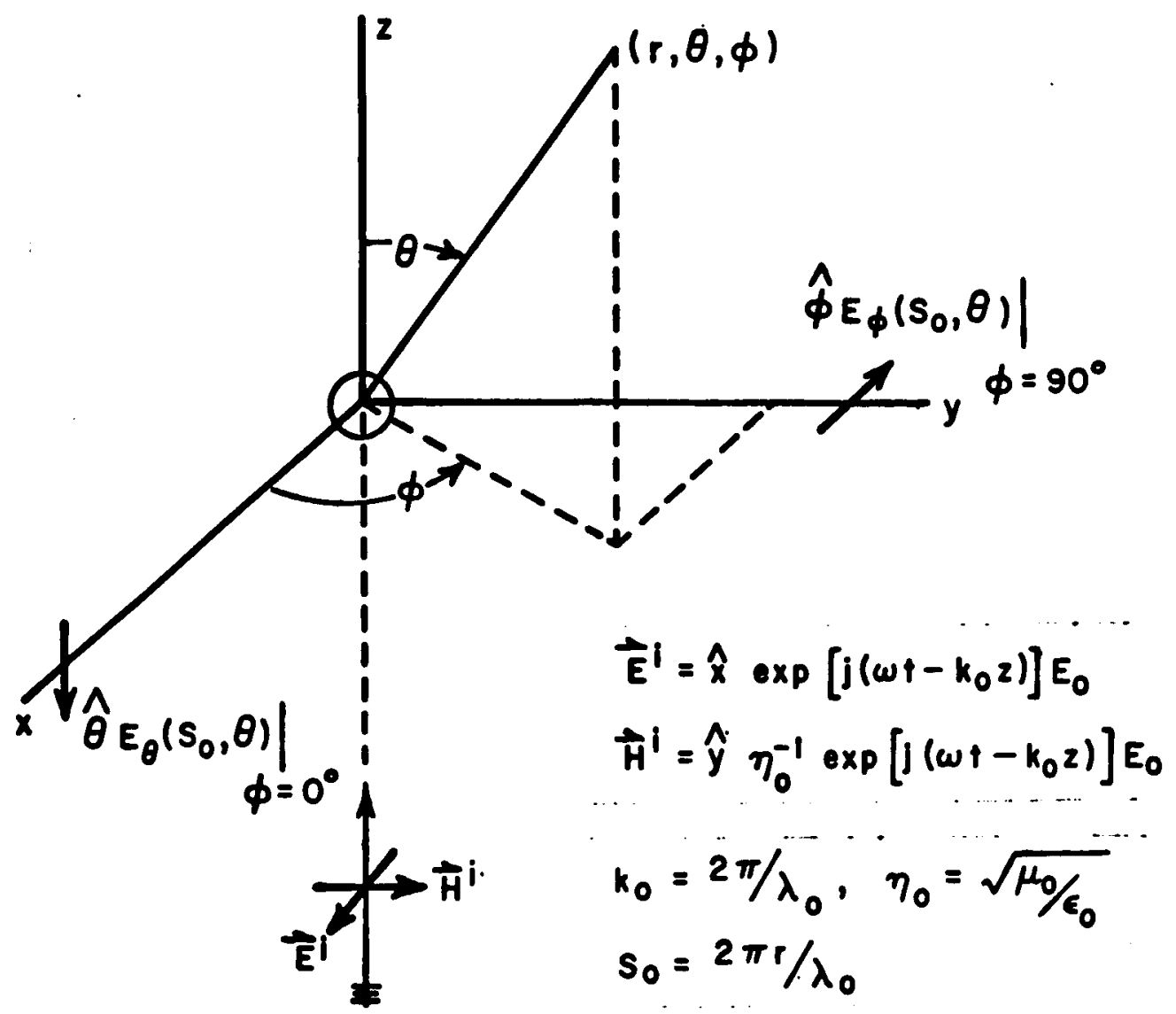

- Fig. 1 --Coordinates systems of a sphere of diameter d illuminated by a homogeneous plane wave.

(ba)

$$
\begin{aligned}
\left.E_{\theta}\left(s_{0}, \theta, p_{0}, \eta_{r}, v_{r}\right)\right|_{\phi=0}= & E_{0} \frac{\exp (j \omega t)}{s_{0}} \sum_{n=1}^{\infty} j^{-n} \\
& {\left[\zeta_{n}\left(s_{0}\right) p_{n}(\theta) a_{n}+j \zeta_{n}^{\prime}\left(s_{0}\right) q_{n}(\theta) b_{n}\right] . }
\end{aligned}
$$

(sb)

$$
\begin{aligned}
\left.E_{\phi}\left(s_{0}, \theta, p_{0}, \eta_{r}, v_{r}\right)\right|_{\phi=90^{\circ}}= & -E_{0} \frac{\exp (j \omega t)}{s_{0}} \sum_{n=1}^{\infty} j^{-n} \\
& {\left[\zeta_{n}\left(s_{0}\right) q_{n}(\theta) a_{n}+j \zeta_{n}^{\prime}\left(s_{0}\right) p_{n}(\theta) b_{n}\right], }
\end{aligned}
$$

where 
(5c)

$$
P_{0}=\pi d / \lambda_{0}
$$

and

$$
s_{0}=2 \pi r / \lambda_{0}
$$

The incident field $E_{0}$ and the spherical coordinates $(r, \theta, \phi)$ are shown in Fig. 1. The parameters $\left(\eta_{r}, v_{r}\right)$ are arguments of the $2^{n}$-pole coefficients $\left(a_{n}, b_{n}\right)$. We omit the superscript $r$ in Eqs. (10) and (11) in p. 565 of Reference 1 and rearrange these coefficients in the form

$$
a_{n}\left(p_{0}, \eta_{r}, v_{r}\right)=a_{n}^{c}\left(p_{0}\right) \frac{\eta_{r}^{-1} X_{n}\left(v_{r} \beta_{0}\right)-A_{n}\left(p_{0}\right)}{\eta_{r}^{-1} X_{n}\left(v_{r} p_{0}\right)-B_{n}\left(p_{0}\right)},
$$

$$
b_{n}\left(\rho_{0}, \eta_{r}, v_{r}\right)=a_{n}^{c}\left(\rho_{0}\right) \frac{\eta_{r} x_{n}\left(v_{r} \rho_{0}\right)-A_{n}\left(\rho_{0}\right)}{\eta_{r} X_{n}\left(v_{r} \rho_{0}\right)-B_{n}\left(\rho_{0}\right)},
$$

where the various functions used are defined in terms of Riccati-Bessel functions $\psi_{n}$ and $\zeta_{n}[9,10]$ as

$$
\begin{aligned}
& A_{n}\left(\rho_{0}\right)=\psi_{n}^{\prime}\left(\rho_{0}\right) / \psi_{n}\left(\rho_{0}\right)=\left(n / \rho_{0}-A_{n-1}\right)^{-1}-n / \rho_{0}, \\
& B_{n}\left(\rho_{0}\right)=\zeta_{n}^{\prime}\left(\rho_{0}\right) / \zeta_{n}\left(\rho_{0}\right)=\left(n / \rho_{0}-B_{n-1}\right)^{-1}-n / \rho_{0}, \\
& X_{n}\left(v_{r} \rho_{0}\right)=\psi_{n}^{\prime}\left(\nu_{r} \rho_{0}\right) / \psi_{n}\left(v_{r} \rho_{0}\right)=\left(n / \nu_{r} \rho_{0}-X_{n-1}\right)^{-1}-n / \nu_{r} \rho_{0},
\end{aligned}
$$


(8a)

$$
a_{n}^{c}\left(\rho_{0}\right)=-\Psi_{n}\left(\rho_{0}\right) / \zeta_{n}\left(\rho_{0}\right)
$$

$$
b_{n}^{c}\left(\rho_{0}\right)=-\psi_{n}^{\prime}\left(\rho_{0}\right) / \zeta_{n}^{\prime}\left(\rho_{0}\right)=a_{n}^{c}\left(\rho_{0}\right) A_{n}\left(\rho_{0}\right) / B_{n}\left(\rho_{0}\right) .
$$

The functions $\psi_{\mathrm{n}}$ and $\zeta_{\mathrm{n}}$ are in turn defined by use of the spherical Bessel functions $j_{n}$ and $y_{n}[4]$ as

$$
\begin{aligned}
& \Psi_{n}(\rho)=\rho j_{n}(\rho), \\
& \zeta_{n}(\rho)=\rho\left(j_{n}(\rho)-j y_{n}(\rho)\right), \\
& \Psi_{n}^{\prime}(\rho)=\frac{d}{d \rho} \psi_{n}(\rho)=\left(\frac{n+1}{\rho}\right) \psi_{n}(\rho)-\Psi_{n+1}(\rho), \\
& \zeta_{n}^{\prime}(\rho)=\frac{d}{d \rho} \zeta_{n}(\rho)=\left(\frac{n+1}{\rho}\right) \zeta_{n}(\rho)-\zeta_{n+1}(\rho),
\end{aligned}
$$

where the argument $p$ is a real or complex number.

Some observations on Eqs. (6) to (8) are in order. If a sphere has the same properties as its surrounding free space, then both $\eta_{r}$ and $\nu_{\mathbf{r}}$ become unity. Consequently, $X_{n}$ in Eq. (7c) becomes identical to $A_{n}$ in Eq. (7a) for all $n$, and both $a_{n}$ and $b_{n}$ in Eq. (6) become identically zero for all $\mathrm{n}$. As a result, there is no scattering from the sphere as it should be the case. If a sphere is perfectly conducting, then $\eta_{r}$ is exactly zero. Consequently, the coefficients in Eq. (6a) and Eq. (6b) reduce to those in Eqs. (8a) and (8b), respectively. For this reason, the coefficients defined in Eq. (8) are called the $2^{n}$-pole coefficients of a perfectly conducting sphere. 
The functions $p_{n}$ and $q_{n}$ used in Eq. (5) are related to the associated Legendre functions [1] $P_{n}{ }^{1}(\cos \theta)$ as

$$
P_{n}(\theta)=\frac{(2 n+1) P_{n}^{1}(\cos \theta)}{n(n+1) \sin \theta}=\left\{\begin{array}{lc}
3 / 2 & \text { for } n=1, \\
(5 / 2) \cos \theta & \text { for } n=2, \\
7\left(5 \cos ^{2} \theta-1\right) / 8 & \text { for } n=3 \\
9 \cos \theta\left(7 \cos ^{2} \theta-3\right) / 8 & \text { for } n=4, \\
\vdots & \vdots
\end{array},\right.
$$

$$
q_{n}(\theta)=\frac{2 n+1}{n(n+1)} \frac{d}{d \theta} P_{n}^{1}(\cos \theta)= \begin{cases}(3 / 2) \cos \theta & \text { for } n=1, \\ (5 / 2)\left(2 \cos ^{2} \theta-1\right) & \text { for } n=2, \\ 7 \cos \theta\left(15 \cos ^{2} \theta-1\right) / 8 & \text { for } n=3, \\ 9\left(28 \cos ^{4} \theta-27 \cos ^{2} \theta+3\right) / 8 & \text { for } n=4, \\ \vdots & \vdots\end{cases}
$$

and

$$
P_{n}(0)=q_{n}(0)=\frac{2 n+1}{2}, \quad n=1,2,3, \ldots,
$$

$$
P_{n}(\pi)=-q_{n}(\pi)=(-1)^{n+1}\left(\frac{2 n+1}{2}\right), n=1,2,3, \ldots .
$$

These functions for $n$ from 1 to 4 are shown in Figs. 2 and 3 to indicate their changes along the bistatic angle $\theta$. At $\theta=\pi$, the components in Eqs. (5a) and (5b) coincide and are called the backscattered field:

$$
\begin{aligned}
\left.E_{\theta}\left(s_{0}, p_{0}, \eta_{r}, v_{r}\right)\right|_{\substack{\phi=0 \\
\theta=\pi}} & \left.E_{\phi}\left(s_{0}, p_{0}, \eta_{r}, v_{r}\right)\right|_{\substack{\phi=\pi / 2 \\
\theta=\pi}} \\
= & -\frac{E_{\theta}}{s_{0}} \sum_{n=1}^{\infty} j^{n}\left(\frac{2 n+1}{2}\right) \\
& {\left[\zeta_{n}\left(s_{0}\right) a_{n}-j \zeta_{n}{ }^{\prime}\left(s_{0}\right) b_{n}\right] . }
\end{aligned}
$$




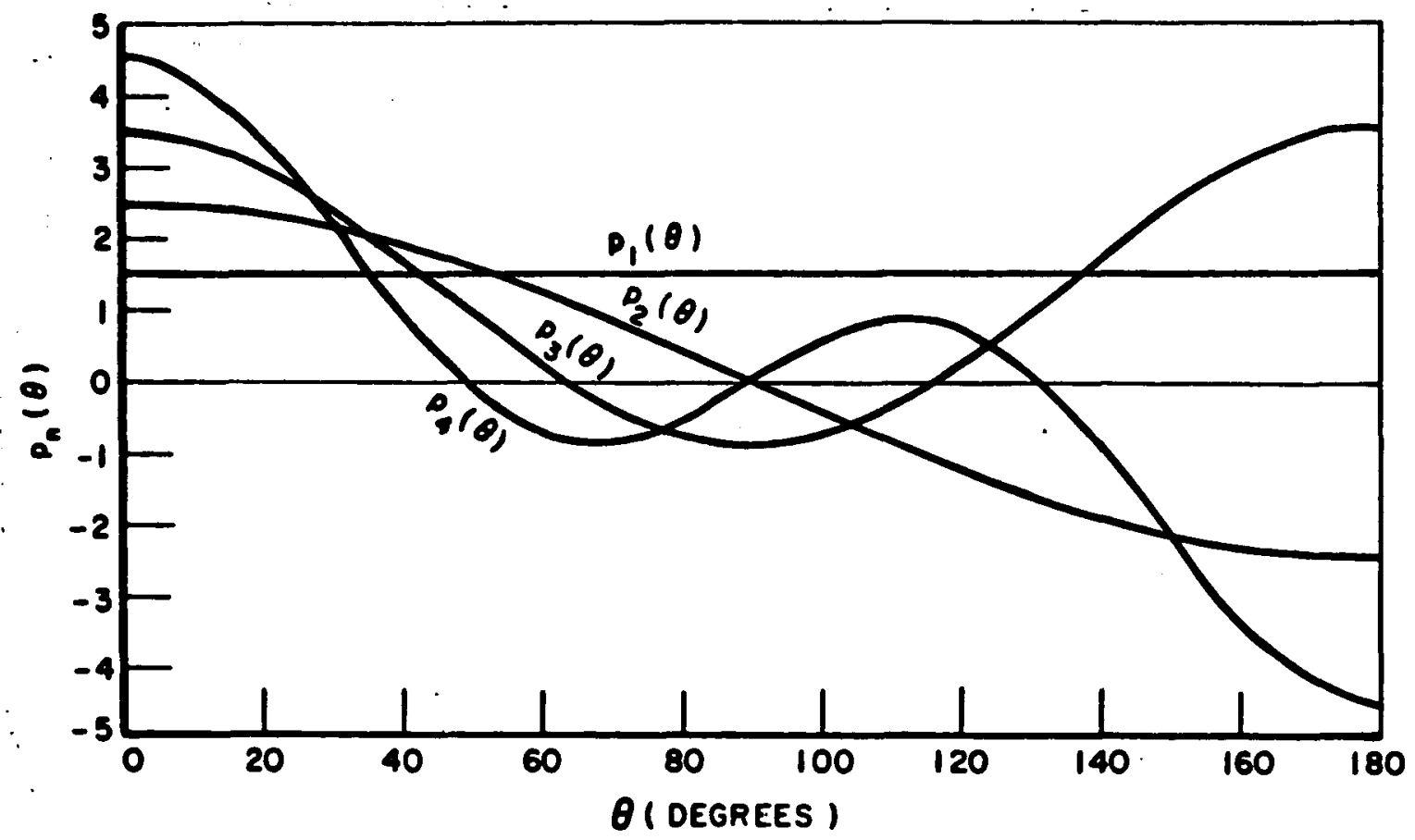

Fig. 2--The functions $p_{1}$ to $p_{4}$ of bistatic angle $\theta$.

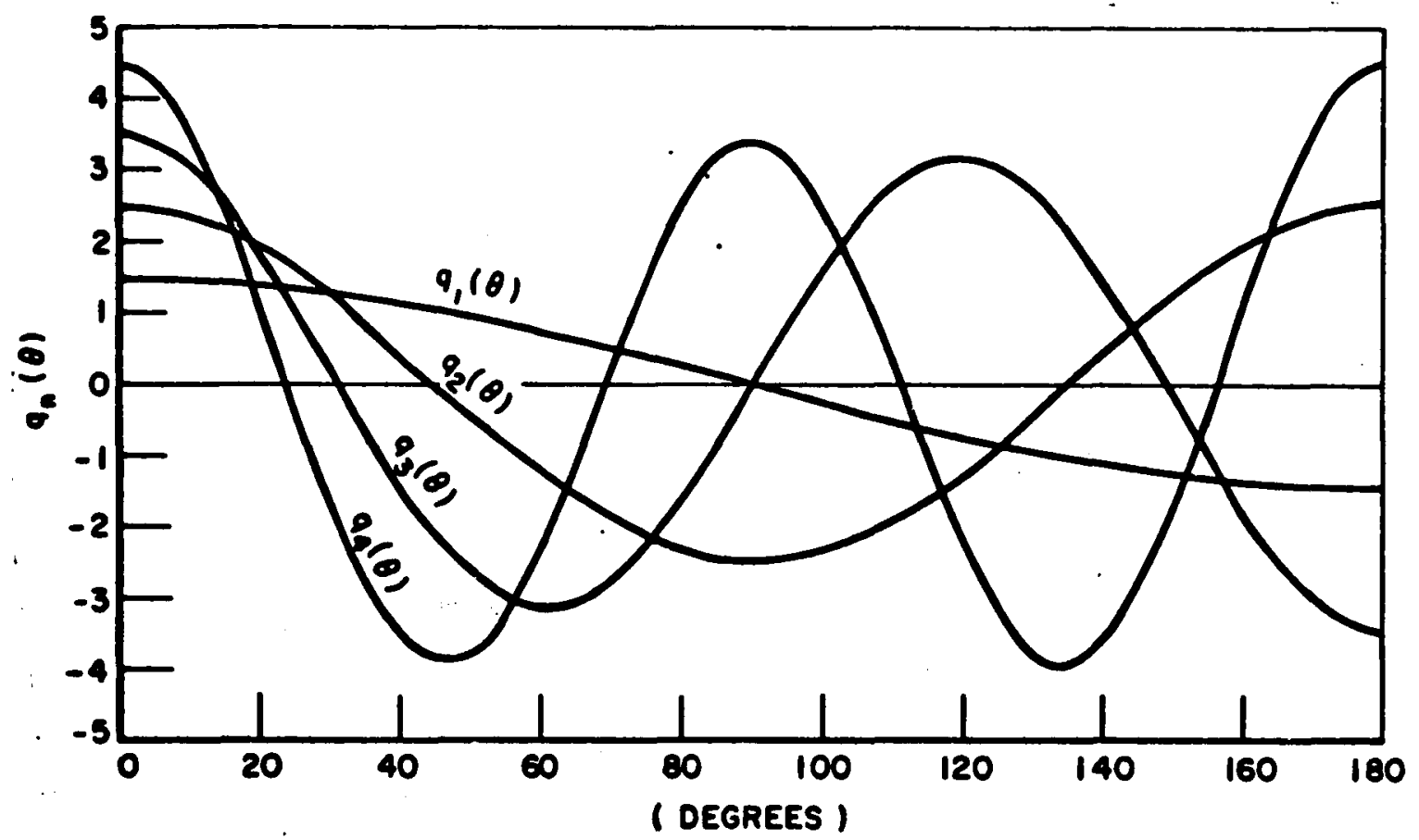

Fig. 3-- The functions of $q_{1}$ to $q_{4}$ of bistatic angle $\theta$. 
When the distance $r$ in Eq. (5d) is sufficiently far from the scattering sphere, one can obtain, by the definitions Eqs. (9b) and (9d), the asymptotic forms:

$$
\zeta_{n}\left(s_{0} \rightarrow \infty\right)=j \zeta_{n}{ }^{\prime}\left(s_{0} \rightarrow \infty\right)=j^{n+1} \exp \left(-j s_{0}\right)
$$

The corresponding far-zone expressions for Eqs. (5) and (11) are then simplified as follows:

$$
\begin{aligned}
& E_{\theta}\left(s_{o} \rightarrow \infty \theta, p_{0}, \eta_{r}, v_{r}\right)=E_{\theta} \frac{\exp \left[j\left(\omega t-s_{o}\right)\right]}{\left(-j s_{0}\right)} \sum_{n=1}^{\infty}\left[p_{n} a_{n}+q_{n} b_{n}\right], \\
& E_{\phi}\left(s_{o} \rightarrow \infty, \theta, f_{0}, \eta_{r}, v_{r}\right)=-E_{\theta} \frac{\exp \left[j\left(\omega t-s_{0}\right)\right]}{\left(-j s_{0}\right)} \sum_{n=1}^{\infty}\left[q_{n} a_{n}+p_{n} b_{n}\right],
\end{aligned}
$$

and

$$
E_{\theta}\left(s_{0} \rightarrow \infty, \rho_{0}, \eta_{r}, v_{r}\right)=-E_{\theta} \frac{\exp \left[j\left(\omega t-s_{0}\right)\right]}{\left(-j s_{0}\right)} \sum_{n=1}^{\infty}(-1)^{n}\left(\frac{2 n+1}{2}\right) .
$$

It is important to note that a perfectly conducting sphere of the same diameter has the same scattered filed components as given in Eqs. (5), (11) and (12), provided all of their $\left(a_{n}, b_{n}\right)$ are replaced by the corresponding coefficients given in Eq. (8). We express these components of a conducting sphere by simply adding a superscript $c$ and eliminating the dependence of the parameters $\eta_{r}$ and $v_{r}, e_{. g}$, the expressions corresponding to Eqs. (12a) and (12b) are 
(13a)

$$
E_{\theta}^{c}\left(s_{o} \rightarrow \infty, \theta, \rho_{o}\right)=E_{o} \frac{\exp \left[j\left(\omega t-s_{o}\right)\right]}{\left(-j s_{0}\right)} \sum_{n=1}^{\infty}\left[p_{n} a_{n}^{c}+q_{n} b_{n}^{c}\right],
$$

and

$$
E_{\phi}^{c}\left(s_{0} \rightarrow \infty, \theta, p_{0}\right)=-E_{0} \frac{\exp \left[j\left(\omega t-s_{0}\right)\right]}{\left(-j s_{0}\right)} \sum_{n=1}^{\infty}\left[q_{n} a_{n}^{c}+p_{n} b_{n}^{c}\right]
$$

B. Normalized Bistatic Fields of a

A sphere is considered small when its diameter is less than $0.5 \lambda$. In the absence of higher-order resonance, all small spheres share a common property, i.e., the dipole coefficients $\left(a_{1}, b_{1}\right)$ defined in Eq. (6) with $n=1$ are the dominant terms in their scattered field components. Some pertinent properties and definitions of three types (dipole, quadrupole and octupole) of small spheres are given in Appendix I.

An octupole sphere is defined to have negligible $\left(a_{n}, b_{n}\right)$ or $\left(a_{n}^{c}, b_{n}^{c}\right)$ with $n$ larger than 3 in its scattered field components. By this definition, the far-zone expressions in Eqs. (12) and (13) with n up to 3 are used to define normalized bistatic fields for an octupole specimen sphere as

(14a)

$$
\gamma_{\theta}=\frac{E_{\theta}\left(s_{0} \rightarrow \infty, \theta, p_{0}, \eta_{x}, v_{r}\right)}{E_{\theta}\left(s_{0} \rightarrow \infty, \theta, p_{0}\right)}=\frac{\sum_{n=1}^{3}\left[p_{n} a_{n}+q_{n} b_{n}\right]}{\sum_{n=1}^{3}\left[p_{n} a_{n}^{c}+q_{n} b_{n}^{c}\right]} \text { in E-plane, }
$$


(14b)

$$
\gamma_{\phi}=\frac{E_{\phi}\left(s_{0} \rightarrow \infty, \theta, p_{0}, \eta_{r}, v_{r}\right)}{E_{\phi}^{c}\left(s_{0} \rightarrow \infty, \theta, p_{0}\right)}=\frac{\sum_{n=1}^{3}\left[\begin{array}{ll}
q_{n} a_{n} & p_{n} b_{n}
\end{array}\right]}{\sum_{n=1}^{3}\left[\begin{array}{ll}
q_{n} a_{n}^{c} & p_{n} b_{n}^{c}
\end{array}\right]} \text { in H-plane, }
$$

and

$$
p_{0}=\pi d / \lambda_{0}<1.5 .
$$

Naturally, the above ratios are valid only when the specimen and the conducting spheres have the same diameter as well as the same illuminating field $E_{o}$ used in Eqs. (12) and (13). When the diameter $d$ of a sphere and the free space wavelength are gauged, the $p_{0}$ in Eq. (14c) is a fixed constant. Also, when a specimen sphere is chosen, the parameters $\left(\eta_{r}, v_{r}\right)$ are complex constants at one particular frequency although they are yet to be determined. After these specifications, the only independent variable remaining in Eqs. (14a) and (14b) is the bistatic angle $\theta$ of the spherical coordinate system shown in Fig. 1.

A quadrupole sphere is defined as one which has only two pairs of significant coefficients, i.e., the summations in Eq. (12) need to include only those terms up to $n=2$ for a specimen sphere, and the same holds true in Eq. (13) for a conducting sphere. The definitions for $\gamma_{\theta}$ and $\gamma_{\phi}$ of a quadrupole specimen sphere are identical to those in Eq. (14), except the summation index now up to $n=2$ instead of 3 . 
Suppose we specifically choose two bistatic angles $\theta_{1}$ and $\theta_{2}$, the principal-plane normalized bistatic fields of a quadrupole specimen sphere may be written at these two angles as

$$
y_{\theta}\left(\theta_{i}\right)=\frac{\sum_{n=1}^{2}\left[p_{n}\left(\theta_{i}\right) a_{n}+q_{n}\left(\theta_{i}\right) b_{n}\right]}{\sum_{n=1}^{2}\left[p_{n}\left(\theta_{i}\right) a_{n}^{c}+q_{n}\left(\theta_{i}\right) b_{n}^{c}\right]} \text { for } i=1 \text { and } 2 \text {, }
$$

$$
\gamma_{\phi}\left(\theta_{i}\right)=\frac{\sum_{n=1}^{2}\left[q_{n}\left(\theta_{i}\right) a_{n}+p_{n}\left(\theta_{i}\right) b_{n}\right]}{\sum_{n=1}^{2}\left[q_{n}\left(\theta_{i}\right) a_{n}^{c}+p_{n}\left(\theta_{i}\right) b_{n}^{c}\right]} \text { for } i=1 \text { and } 2 \text {, }
$$

and

$$
P_{0}=\pi d / \lambda_{0}<1.0
$$

Note that the diameter $d$ of a quadrupole sphere is required to satisfy Eq. (15c). When $p_{0}$ becomes a constant from a set of specified $d$ and $\lambda_{0}$, the coefficients $\left(a_{n}^{c}, b_{n}^{c}\right)$ defined in Eq. (8) can be computed for use in Eqs. (15a) and (15b). Also, when $\theta_{1}$ and $\theta_{2}$ are specified, the values of $p_{n}$ and $q_{n}$ can be readily computed by use of Eq. (10). Finally, the values of $\gamma_{\theta}$ and $\gamma_{\phi}$ at $\theta_{1}$ and $\theta_{2}$ can be obtained by actually performing 
measurements on the scattered field components of both specimen and conducting spheres. It is now evident that we have in Eqs. (15a) and (15b) only $\left(a_{1}, b_{1}\right)$ and $\left(a_{2}, b_{2}\right)$ remaining as undetermined coefficients. Our problem here is to determine these coefficients, and subsequently solve for the desired parameters $\left(\mu_{r}, \epsilon_{r}\right)$.

The far-zone normalized bistatic fields defined above may also be applied to a dipole sphere $\left(p_{0}<0.2\right)$ which has only one pair of significant coefficients $\left(a_{1}, b_{1}\right)$. One exception for a dipole specimen is that its near-zone field componetns also can be measured to determine $\left(\mu_{r}, e_{r}\right)$. Results of actual measurements on dipole specimens have been reported by Young [11] and Spentzos . [12]

The remaining part of the present investigation will be concentrated on the study of quadrupole specimens. C. Steps In Determination of $\left(\mu_{\left.x, C_{r}\right) \text { From }}\right.$

The procedure of obtaining the parameters $\left(\mu_{r}, \epsilon_{r}\right)$ from measured scattered field components is outlined here to give the reader a perspective of inverse scattering problems. Details involved in the following steps are to be treated in later chapters:

1. Use of a quadrupole specimen for measurements requires that, for a chosen frequency range, the diameter of the specimen sphere satisfies the inequality

$$
P_{0}=\pi d / \lambda_{0}<1.0
$$


For instance, we choose 6 and $7 \mathrm{GHz}$ for our measurements and the diameter of both conducting and specimen spheres is made equal to $1.27 \mathrm{~cm}$ which satisfies the above inequality.

2. The specimens to be measured are required to be homogeneous and isotropic.

3. To obtain the normalized E-plane bistatic fields $\gamma_{\theta}\left(\theta_{i}\right)$ and the normalized $H$-plane bistatic fields $\gamma_{\phi}\left(\theta_{\mathfrak{i}}\right)$, measurements in both planes are required to give the ratios: $\gamma_{\theta}\left(\theta_{\mathfrak{i}}\right)=\frac{\text { The specimen sphere's } E_{\theta} \text { measured at } \theta_{\mathfrak{i}} \text { in the } E \text {-plane }}{\text { The conducting spher's } E_{\theta}^{c} \text { measured at } \theta_{\mathfrak{i}} \text { in the } E \text {-plane }}$, $\gamma_{\phi}\left(\theta_{i}\right)=\frac{\text { The specimen sphere's } E_{\phi} \text { measured at } \theta_{i} \text { in the } H \text {-plane }}{\text { The conducting sphere's } E_{\phi}^{C} \text { measured at } \theta_{i} \text { in the } H \text {-plane }}$, where $i=1,2$. Only two angles $\theta_{1}$ and $\theta_{2}$ in both planes are needed for measurements on quadrupole spheres.

4. Substitute the values of $y^{\prime} s$ obtained in step 3 into Eq. (15) to form a system of four simultaneous linear equations

(17a)

$$
\begin{aligned}
& p_{1}\left(\theta_{1}\right) a_{1}+q_{1}\left(\theta_{1}\right) b_{1}+p_{2}\left(\theta_{1}\right) a_{2}+q_{2}\left(\theta_{1}\right) b_{2} \\
& =\gamma_{\theta}\left(\theta_{1}\right) \sum_{n=1}^{2}\left[p_{n}\left(\theta_{1}\right) a_{n}^{c}+q_{n}\left(\theta_{1}\right) b_{n}^{c}\right], \\
& q_{1}\left(\theta_{2}\right) a_{1}+p_{2}\left(\theta_{1}\right) b_{1}+q_{2}\left(\theta_{1}\right) a_{2}+p_{2}\left(\theta_{1}\right) b_{2} \\
& =\gamma_{\phi}\left(\theta_{1}\right) \sum_{n=1}^{2}\left[q_{n}\left(\theta_{1}\right) a_{n}^{c}+p_{n}\left(\theta_{1}\right) b_{n}^{c}\right] .
\end{aligned}
$$


(17c)

$$
\begin{aligned}
p_{1}\left(\theta_{2}\right) a_{1} & +q_{1}\left(\theta_{2}\right) b_{1}+p_{2}\left(\theta_{2}\right) a_{2}+q_{2}\left(\theta_{2}\right) b_{2} \\
& =\gamma_{\theta}\left(\theta_{2}\right) \sum_{n=1}^{2}\left[p_{n}\left(\theta_{2}\right) a_{n}^{c}+q_{n}\left(\theta_{2}\right) b_{n}^{c}\right],
\end{aligned}
$$

and

$$
\begin{aligned}
q_{1}\left(\theta_{2} \lambda a_{1}\right. & +p_{1}\left(\theta_{2}\right) b_{1}+q_{2}\left(\theta_{2}\right) a_{2}+p_{2}\left(\theta_{2}\right) b_{2} \\
& =Y_{\phi}\left(\theta_{2}\right) \sum_{n=1}^{2}\left[q_{n}\left(\theta_{2}\right) a_{n}^{c}+p_{n}\left(\theta_{2}\right) b_{n}^{c}\right]
\end{aligned}
$$

Since $p_{n}$ and $q_{n}$ are known when $\theta_{1}$ and $\theta_{2}$ are specified and $a_{n}^{c}$ and $b_{n}^{c}$ are also known when $\rho_{0}$ is determined, the system of equations can be used to solve for the undetermined coefficients $\left(a_{1}, b_{1}\right)$ and $\left(a_{2}, b_{2}\right)$ of a specimen sphere.

5. The solutions $\left(a_{1}, b_{1}\right)$ and $\left(a_{2}, b_{2}\right)$ obtained above are substituted into $\mathrm{Eq} \cdot(6)$ to solve for $\eta_{\mathrm{r}}$ and $\mathrm{X}_{\mathrm{n}}$ as

$$
\eta_{r}=+\sqrt{\frac{\left(a_{n}-a_{n}^{c}\right)\left(b_{n}-b_{n}^{c}\right)}{\left(a_{n}-b_{n}^{c}\right)\left(b_{n}-a_{n}^{c}\right)}} \quad, \quad n=1,2 \ldots
$$

and

$$
x_{n}=+B_{n} \sqrt{\frac{\left(b_{n}^{c}-a_{n}\right)\left(b_{n}^{c}-b_{n}\right)}{\left(a_{n}-a_{n}^{c}\right)\left(b_{n}-a_{n}^{c}\right)}}, n=1,2 \ldots
$$

where $B_{n}\left(p_{0}\right)$ is defined in Eq. $(7 b)$. We denote $\eta_{r}\left(a_{1}, b_{1}\right)$ and $\eta_{r}\left(a_{2}, b_{2}\right)$ for $n=1$ and 2, respectively, in Eq. (16a). If $\left(a_{n}, b_{n}\right)$ were theoretical values of a specimen sphere, it is 
required that $\eta_{r}\left(a_{1}, b_{1}\right)$ is identical to $\eta_{r}\left(a_{2}, b_{2}\right)$. However, because of truncation errors in Eq. (15), the consistency of $\eta_{x}$ would never be exactly satisfied even if exact values of $\gamma$ 's were used in Eq. (17).

6. The complex number obtained for $X_{1}$ in Eq. (18b) is used to determine the refractive index of a specimen. If the value of $\mathrm{X}_{1}$ were known exactly, it is sufficient to use the refractive index chart described in the next chapter to solve for the appropriate value of $v_{\mathrm{r}} \mathrm{Po}_{\mathrm{O}}$. The solution for the refractive index $v_{r}$ is then obtained by dividing the solution with the known value $\rho_{0}$. Similar to step 5 , the value of $X_{2}$ in Eq. (18b) is also used in the consistency process described in Chapter IV.

7. The valies of $\left(\eta_{Y}, v_{r}\right)$ resulted from application of the consis tency process are then used to give $\left(\mu_{Y}, \epsilon_{Y}\right)=\left(v_{Y} \eta_{Y}, v_{Y} \eta_{I}^{-1}\right)$ We note here that, if one could tolerate the truncation errors in Eq. (15) and measurement inaccuracies to be discussed in Cahpter V, steps 5 to 7 could be performed without use of the consistency process. In other words, one set of four $\gamma^{\prime} s$ can be used in these steps to result a set of solution $\left(\eta_{r}, v_{r} p_{O}\right)$. 


\section{A. A Transcendental Equation With Complex Roots}

The refractive index chart to be described here is constructed primarily for finding the refractive index of a specimen sphere. As defined in Eq. (7c) with $n=1, X_{1}\left(\nu_{r} P_{0}\right)$ is a complex transcendental function with a complex argument $v_{\mathrm{r}} \rho_{\mathrm{O}}$. After $\mathrm{X}_{1}$ is determined from measurements, the main task is to find the appropriate complex number $v_{x} P_{0}$ so that the refractive index of a specimen can be determined. If substitutions of Eqs. (9a) and (9c) are made to Eq. (7c) with $n=1$, and the Riccati-Bessel functions[10] are then replaced by trigonometric functions, one can obtain $X_{1}$ in terms of tangent function as

$$
\begin{aligned}
X_{1}\left(v_{r} \rho_{0}\right) & =\frac{\left(v_{r} \rho_{0}\right) \tan \left(v_{r} \rho_{0}\right)}{\tan \left(v_{r} \rho_{0}\right)-v_{r} \rho_{O}}-\frac{1}{v_{r} \rho_{O}} \\
& =\operatorname{Re}\left(X_{1}\right)+j \operatorname{Im}\left(X_{1}\right) \\
& =X_{1}^{\prime}+j X_{1}^{\prime \prime}
\end{aligned}
$$

where

$$
v_{r} \rho_{0}=\left(v_{r}^{\prime}+j v_{r}^{\prime \prime}\right) \rho_{0}
$$


The prime and double prime used in Eq. (19) are not to be confused with derivatives. For passive media, the real part of $v_{r} \rho_{O}$ is always positive while the imaginary part is always negative. Figures 4 and 5 indicate the portion of the $v_{r} \rho_{0}$-plane, $0 \leq v_{r}^{\prime} \rho_{0} \leq 15$ and $0 \geq v_{r} " \rho_{0} \geq-8$, for which the refractive index chart has been constructed for the investigation. As $v_{X} \rho_{0}$ changes, the real pert of $X_{1}$ may assume any value while the imaginary part of $X_{1}$ must remain positive. Figure 6 shows the region of the $\mathrm{X}_{1}$-plane, $-\infty<\mathrm{X}^{\prime}<+\infty$ and $0 \leq \mathrm{X}^{\prime \prime}<\infty$, which one may find in the refractive index chart. Some principal $\mathrm{X}_{1}{ }^{\prime}$-contours and $\mathbf{X}_{1}{ }^{\prime \prime}$-contours are shown in Fig. 4 with solid and dashed curves, respectively. In the region $2<v_{\mathbf{r}}{ }^{\prime} \rho_{0}<15$ and $0>v_{\mathbf{r}}{ }^{\prime} \rho_{0}>-3$, the $\mathrm{X}_{1}{ }^{\prime}-$ contours of +0.1 and -0.1 are seen to repeat themselves almost periodically, and similarly so are the $X_{1}^{\prime \prime}$-contours of $0.5,1.0$, etc. The nature of repetitive occurrence by a region of $\mathrm{X}_{1}$ is more clearly revealed by Fig. 5. For example, the regions designated by $1-0$ to $1-4$ in Fig. 5 all correspond to a single region of $X_{1}, 0.05 \leq X_{1}{ }^{\prime}<\infty$ and $1.0 \leq \mathrm{X}_{1} "<\infty$, which is shown in region 1 of Fig. 6 . In other words, for one value of $X_{1}$ in region 1 of Fig. 6 one should be able to find five values of $v_{r} p_{0}$ in the regions 1-0 to 1-4 of Fig. 5. By use of Fig. 4, one can approximately locate the first five roots of $x_{1}=0.1+j 1.0$ as $v_{x} P_{0}=2.3-j 2.9,5.1-j 1.6,8.4-j 1.5,11.6-j 1.5$, and $14.8-j 1.5$. These roots are found by locating those points at which the $\mathrm{X}_{1}{ }^{\prime}$-contour of 0.1 intersect with the $X_{1}$ "- contour of 1.0 . Selection of an appropriate 


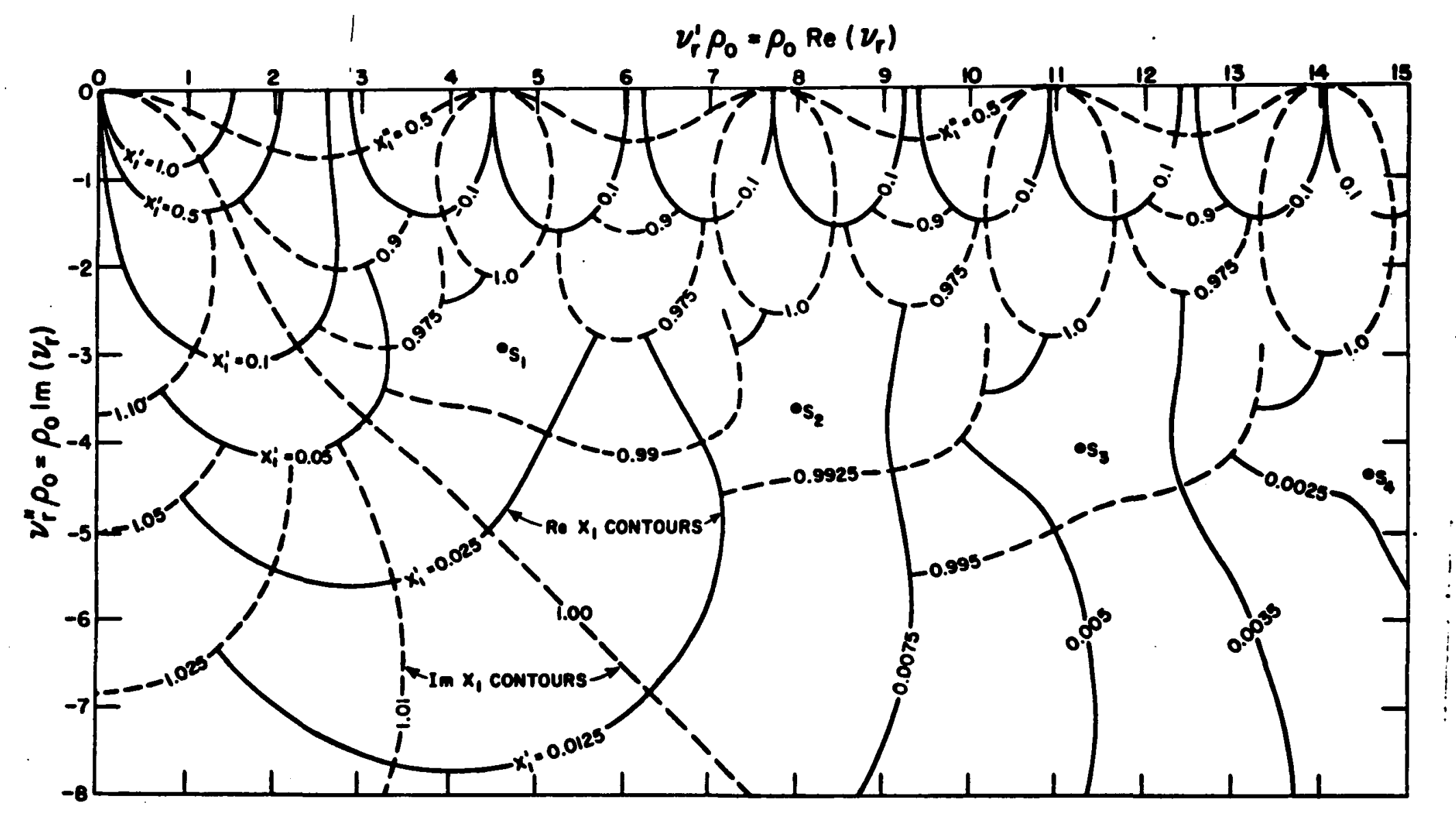

Fig. 4--Some principal contours of $X_{1}\left(v_{r} P_{0}\right)$ in the $v_{r} P_{0}-p l a n e$. 


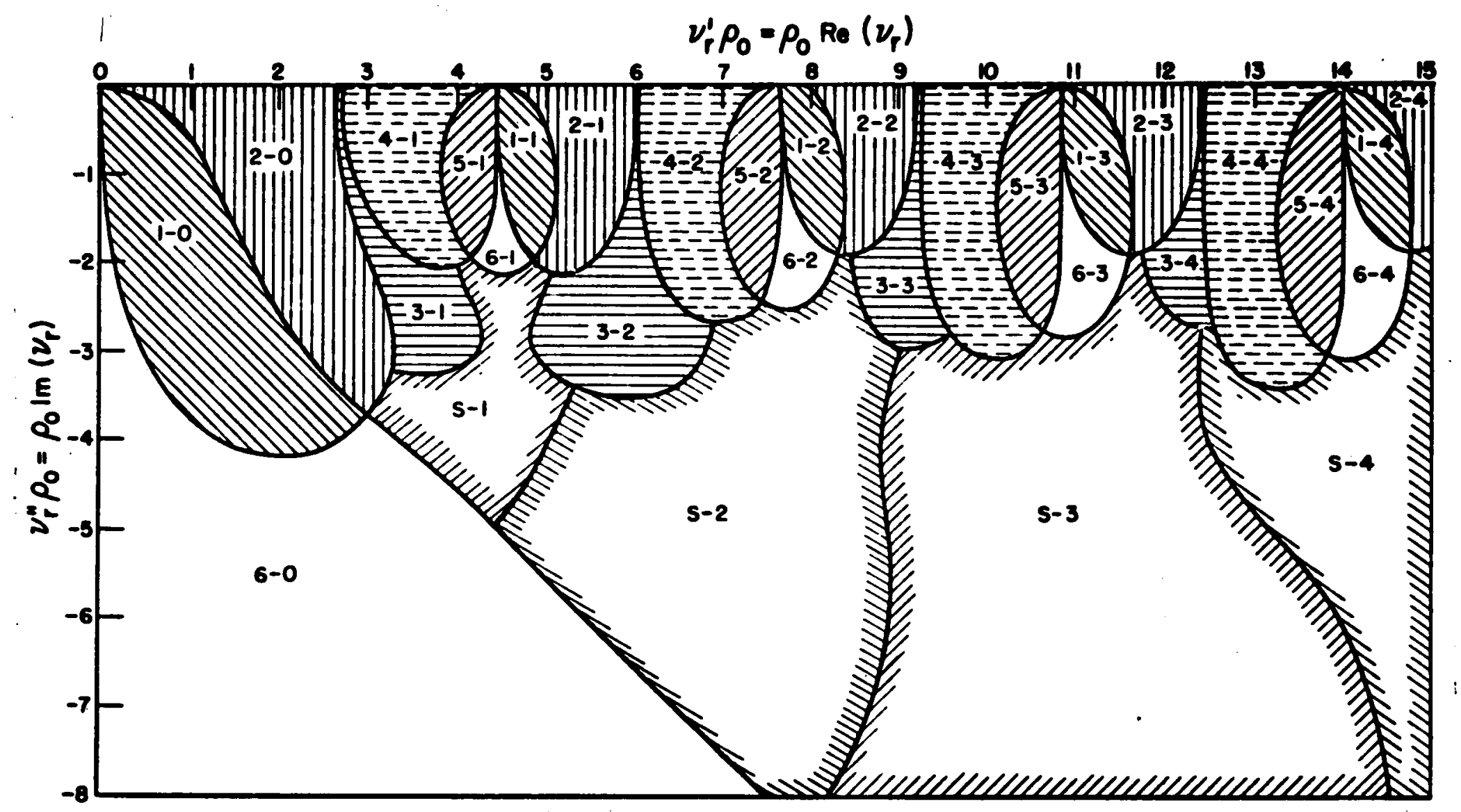

Fig. 5--Six repetitive regions and four saddle point regions of $X_{1}\left(v_{r} P_{0}\right)$ in the $v_{r} P_{0}-p l a n e$. 


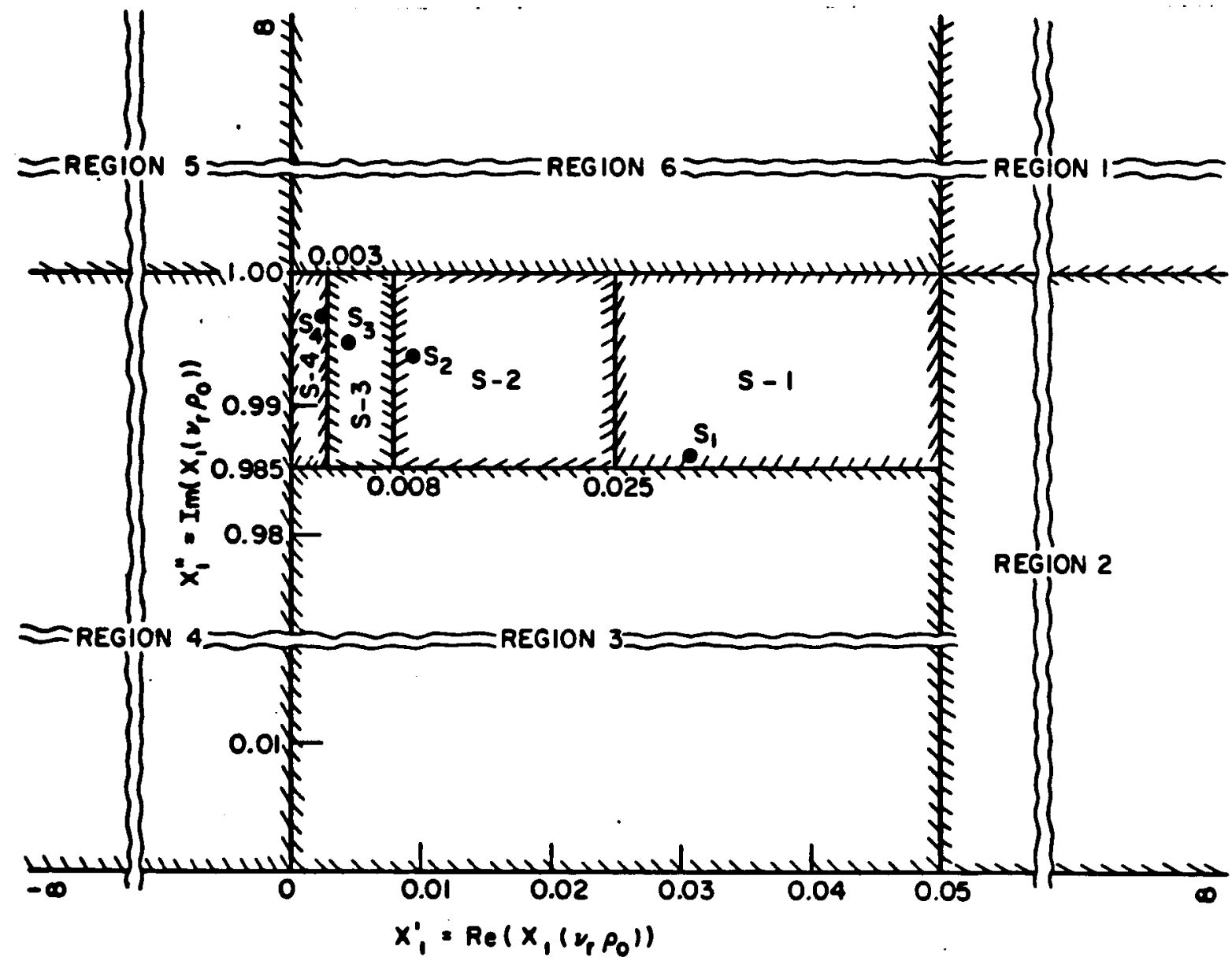

Fig. 6. The $\mathrm{X}_{1}$-plane representation of those regions in Fig. 5 .

root and details of the chart for more accurate roots are to be discussed in the latter parts of this chapter.

In Fig. 4 one can see that the rates of change become smaller and smaller when $v_{r} " p_{0}$ is less than -3 . The points $S_{1}$ to $S_{4}$ in the figure are four saddle points at which $X_{1}$ has zero rate of change. In their neighborhoods, one single value of $\mathrm{X}_{1}$ always has double roots in 
$v_{r} P_{0}$. This fact becomes clear when one examines the manner in which four values of $X_{1}$ in Fig. 7 are transformed into Fig. 8 by use of the refractive index chart to yield eight values of $v_{r} p_{0}$. For instance, $X_{1}=0.024+j 0.98$ of Fig. 7 has two roots about $v_{x} P_{0}=4.0-j 2.5$ and $5.6-$ j 3.0 in Fig. 8. Consequently, it would be ambiguous as to which of the two roots is appropriate, whenever the value of $X_{1}$ is in the immediate neighborhood of a saddle point.

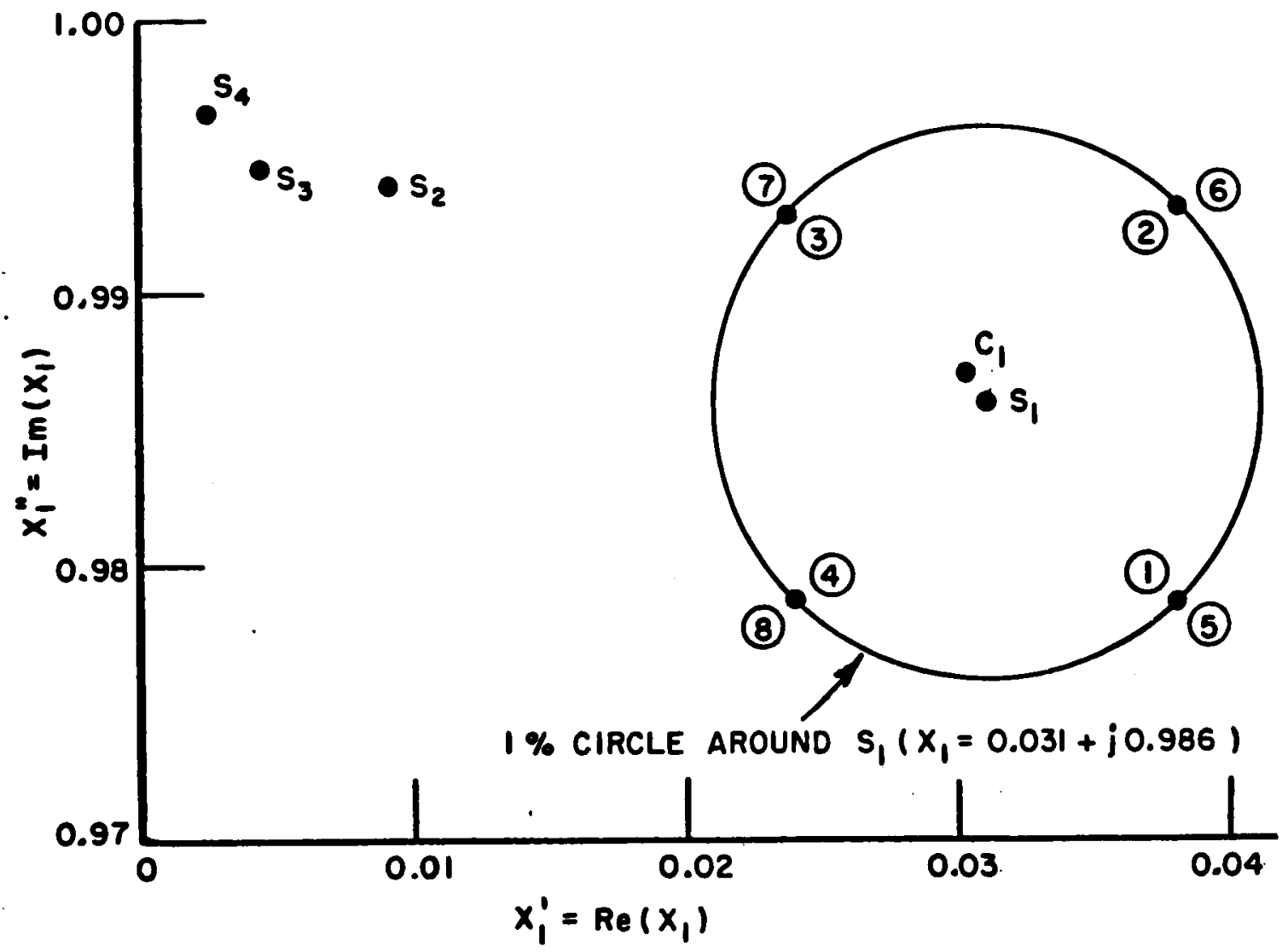

Fig. 7--A $1 \%$-circle centered at $S_{1}$ in the first saddle point region of $\mathrm{X}_{1}$-plane. 


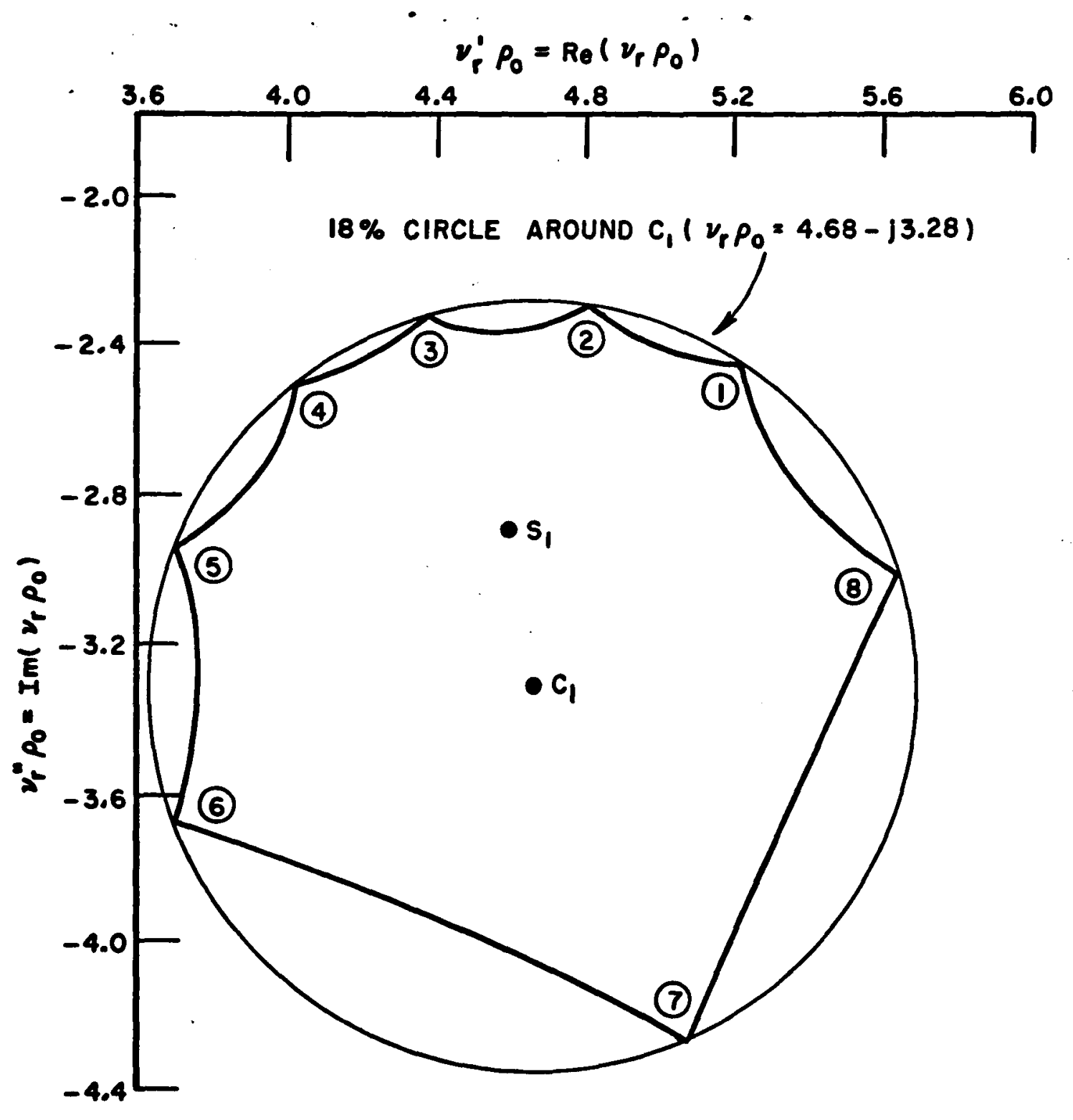

Fig. 8--The 18\%-circle centered at $C_{1}$ of $v_{r} p_{0}$-plane corresponding to the $X_{1}$-plane of Fig. 7 . 
When the imaginary part of $v_{\mathbf{r}} p_{\mathrm{O}}$ becomes smaller than the coordinates of those saddle points shown in Fig. 4, one can see from the figure or by expanding $E q .(19 a)$ for large $v_{r}^{\prime \prime} p_{o}$ that $X_{1}\left(v_{r} p_{o}\right)$ becomes a monotonic function with very small rates of change. In this case there is always a unique root for $X_{1}\left(\nu_{r} P_{0}\right)$. However, a sphere becomes less and less penetrable when $\nu_{r} " p_{0}$ is smaller.

\section{B. The Chart and the Appropriate Root}

Originally the refractive index chart was constructed[13] with an area of $30^{\prime \prime} \times 20^{\prime \prime}$ covering the complex plane $0 \leq \nu_{r}^{\prime} \rho_{0} \leq 6.0$ and $0 \geq v^{\prime \prime} p_{0} \geq-4.0$. Its scaled-down version can be found in Reference 13. The present version is extended to cover the portion of the $v_{r} \rho_{0}$-plane shown in Fig. 4 or 5. The detailed contour plots are given in Appendix II. Figure 9 indicates the extent covered by individual figures. We note here that most small spheres of typical media are covered by the first two figures. The rest of the figures cover mdeia of large conductivity or large refractive index.

We have mentioned earlier that five roots can be found in Fig. 4 for $\mathrm{X}_{1}=0.1+\mathrm{j} 1.0$. In fact there are infintely many roots for this value of $X_{1}$. The appropriate root of a value of $X_{1}$ depends upon the value of the relative intrinsic impedance of a particular specimen. Recall that our media is passive and, as a consequence, we can have only negative imaginary parts for both $\mu_{x}$ and $\epsilon_{r}$. Therefore, for a 


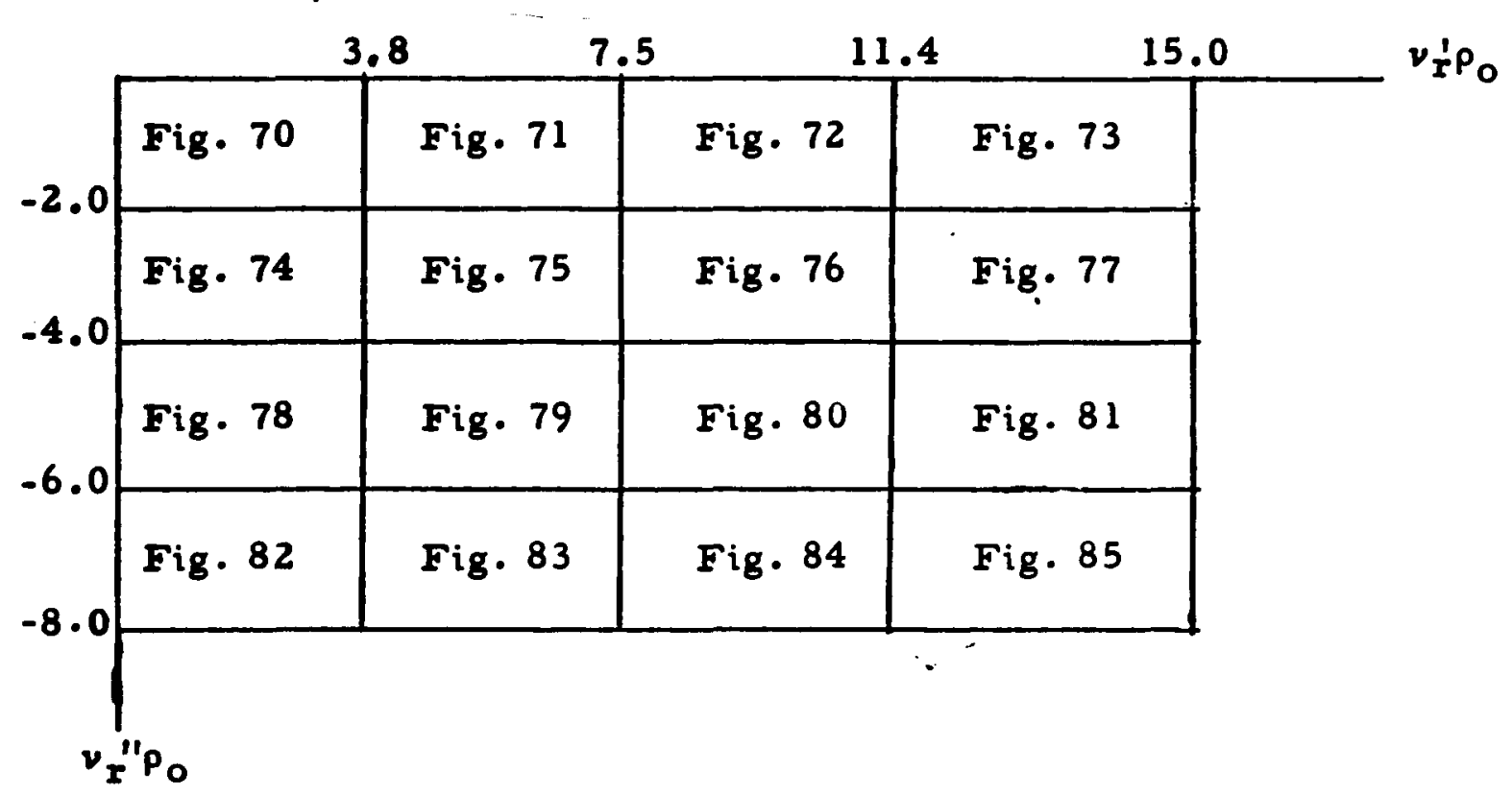

$$
\begin{aligned}
& X_{1}\left(v_{r} P_{0}\right)=\operatorname{Re}\left(X_{1}\right)+j \operatorname{Im}\left(X_{1}\right)=X_{1}^{\prime}+j X_{1}^{\prime} \\
& \nu_{r} \rho_{0}=\nu_{r}^{\prime} \rho_{0}+j \nu_{r}^{\prime \prime} \rho_{0} \text { where } \rho_{0}=\pi d / \lambda_{0} \text { and } v_{r}=\sqrt{\mu_{r} \epsilon_{r}} \\
& \text { The contours of } X_{1}\left(v_{Y} \rho_{O}\right) \text { are plotted in these figures of } \\
& \text { the complex plane } v_{r} p_{0}
\end{aligned}
$$

Fig. 9--Outline of the figures which give in Appendix II the detailed contour plots of the refractive index chart. 
specified value of $\eta_{r}$, the appropriate root $\nu_{r} p_{O}$ is the one that would give, by use of $E_{q}$. (4), a pair of $\left(\mu_{r}, \epsilon_{r}\right)$ that have a negative imaginary part.

For example, if we have a specimen sphere of diameter $d=1.27$ $\mathrm{cm}$ which is measured at a frequency $f=6.17 \mathrm{GHz}$, then $p_{0}=0.82112$ by Eq. (15c). Suppose measurements on the specimen had produced a pair of values $\left(\eta_{r}, X_{1}\right)=(0.4179-j 0.09713,-0.3300+j 0.8995)$, then the task is to find an appropriate root for the $X_{1}$ so that $\left(\mu_{r}, \in_{r}\right)$ of the specimen can be determined. We first take a look at Fig. 4 and note that the $X_{1}^{\prime}$-coutours with negative sign occur only in the region where $-\nu_{\mathbf{r}}{ }^{\prime} P_{0}$ is small. Figure 9 indicates that Figs. 70 to 73 in Appendix II cover the region $0 \geq v_{\mathbf{r}} " \rho_{0} \geq-2$. Figure 70 is reproduced in Fig. 10 for illustration. While the $\mathrm{X}_{1}$ "-contour of 0.9 can be directly located in the figure, the $X_{1}{ }^{\prime}$-contour of -0.33 needs be located by interpolation on the contours of -0.30 and -0.35 . The intersection of these two contours is at $v_{\mathrm{r}} \mathrm{P}_{\mathrm{O}}=3.7-\mathrm{j} 0.9$ as shown in Fig. 10. Dividing this value by $P_{0}=0.82112$ we obtain $\nu_{r}$ together with $\eta_{r}$ as $\left(\eta_{r}, v_{r}\right)=(0.4179-j$ $0.09713,4.506-j 1.096)$. The resulting $\left(\mu_{r}, e_{r}\right)=\left(\eta_{r} v_{r}, v_{r} / \eta_{r}\right)$ are complex numbers both with a negative imaginary part. Therefore, we consider $v_{\mathrm{I} P_{\mathrm{O}}}=3.7-\mathrm{j} 0.9$ as an appropriate root for the value of $\mathrm{X}_{1}$. Is this the appropriate root for the specimen under consideration? The answer is yes, because the rest of possible roots, e.g., $v_{x} P_{O}=6.89-$ j 0.88 in Fig. 71 , all produce a positive imaginary part for $e_{x}$. 


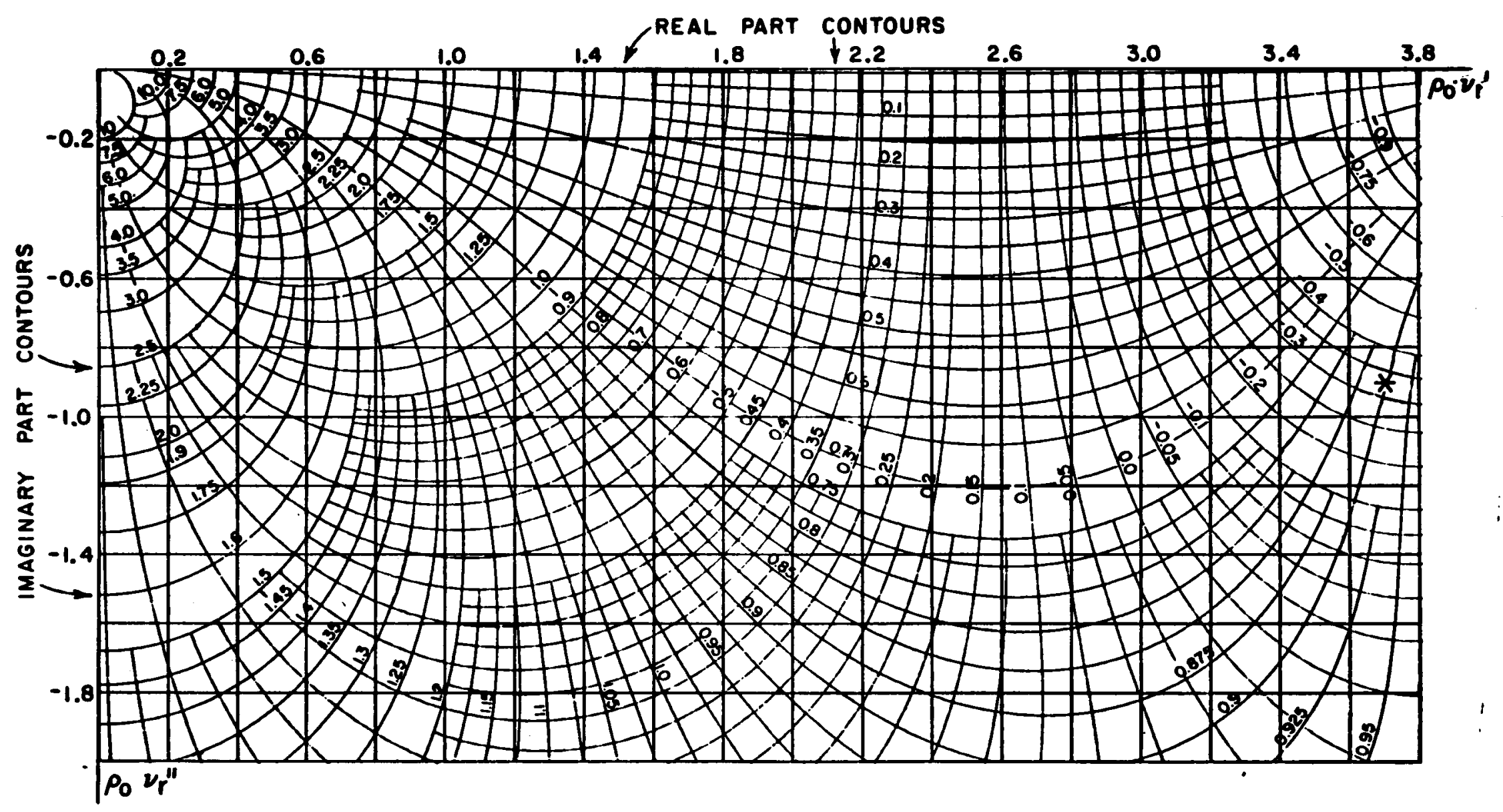

A root for $X_{1}=-0.33+j 0.9$ is at $\nu_{r} \rho_{0}=3.7-j 0.9$ as marked by *

Fig. 10--A portion of the chart used for numerical illustrations. 


\section{Inaccuracies And Limitations}

When a value of $\nu_{\mathbf{r}} \rho_{0}$ is given, it is in general possible to find $X_{1}$ from the refractive index chart with an accuracy to the third significant digit by proper interpolation. There are two extreme exceptions. On the one hand, an accuracy to the fifth or even the sixth significant digits can be obtained for $X_{1}$ when $v_{x} \rho_{0}$ is in the neighborhood of a saddle point. On the other hand, when $-v_{Y} " p_{O}$ is small it may be difficult in certain regions to obtain even an accuracy to the first digit. Consequently, as in the use of a Smith chart, there are regions which give less accurate results than the others in the refractive index chart.

When a value of $X_{1}$ is given, one may find $v_{r} P_{0}$ in general accurate to the second decimal place. If the original version, instead of the scaled-down version, is used, it is possible to obtain $v_{Y} p_{O}$ accurate to the third decimal place. It is obvious that the percentage inaccuracy for the answer $v_{r} p_{0}$ decreases with increasing $v_{r} p_{O}$. The reason is simply because errors take place at a fixed decimal place. When $\mathbf{X}_{1}$ is in the region where $\nu_{x}^{\prime \prime} p_{0}>-0.1$, the value found for the imaginary part of $v_{r} p_{O}$ is highly uncertain. This means that our refractive index is not capable of determining accurately the loss components of extremely low-loss media. Should one decide to use the present method to determine the values of $\left(\mu_{x}, c_{x}\right)$ for an extremely low-loss medium, the portion of the refractive index chart in $0 \geq v_{r}^{\prime \prime} P_{0} \geq-0.1$ needs to be expanded and it would be essential to measure the phase angle of the 
scattered fields with much greater accuracy. Since we emphasize the present method for lossy specimens, no expansion of the portion of the chart is made.

The real limitation of the present method can be easily visualized through use of the refractive index chart. It involves the question of how accurate one can determine $X_{1}$ from measured data. Should measurements give a value of $X_{l}$ uncertain to less than $1 \%$, one may ask: How uncertain is the value obtained for $v_{r} \rho_{0}$ ? The answer to this question is that the uncertainty of the solution increases when $v_{r}^{\prime \prime} P_{0}$ is smaller than -3 . Figures 7 and 8 provide an example showing an $18 \%$ inaccuracy or uncertainty for $\nu_{r} P_{0}$ when its imaginary part is in the neighborhood of the saddle point. Therefore, for the purpose of the present method, one may consider a sphere less and less penetrable when the usual skin depth is less than the radius or specifically when $v_{x}^{\prime \prime} p_{0}$ becomes smaller than -3 . This implies that energy does not propagate completely through the sphere. In fact, under this circumstance, the $\tan \left(v_{\mathrm{r}} \rho_{\mathrm{O}}\right)$ in $\mathrm{Eq} \cdot(16 \mathrm{a})$ can be simply replaced by $(-\mathrm{j})$ and the expression $\mathrm{X}_{1}$ is greatly simplified. We conclude this section by noting that, unless $X_{1}$ could be obtained accurately to the fourth significant digit, no accurate $\nu_{\mathbf{r}} \rho_{0} c a n$ be obtained in the region $-3 \geq v_{r}^{\prime \prime} \rho_{0}$ of our refractive index chart. Other useful applications of the chart can be found in Appendix II. 


\section{CHAPTER IV \\ THE CONSISTENCY PROCESS}

Numerical examples are to be given here to illustrate the consistency process. The process is to improve the accuracy with which the acutal values of $\left(\eta_{r}, v_{r}\right)$ can be obtained from a knowledge of approximate values of $\left(\gamma_{\theta}, Y_{\phi}\right)$ defined in Eq. (15) or Eq. (16). The values of $\gamma^{\prime} s$ defined in Eq. (16) are approximate because experimental errors are involved. On the other hand, truncation errors are involved in the $\gamma^{\prime} s$ defined by Eq. (15). These two kinds of errors are inseparable and take place simultaneously in the system of Eq. (17) from which the coefficients $\left(a_{1}, b_{1}\right)$ and $\left(a_{2}, b_{2}\right)$ are to be solved. We shall calculate the exact values of $\gamma^{\prime}$ s by a set of specified parameters $\left(\theta, p_{0}, \eta_{r}, v_{r}\right)$ and then these $\gamma^{\prime}$ 's are used in Eq. (17) for the inverse problem.

\section{A. The Uncertainty Circle In The $\gamma$-Plane}

Let us use a sphere with $d=1.27 \mathrm{~cm}$ as an example. For $f=7.03 \mathrm{GHz}$, the value of $p_{0}$ is 0.93557 . If the parameters $\left(\eta_{r}, v_{r}\right)=$ $(0.2783-j 0.1057,5.550-j 2.170)$ are used, the exact values of $\gamma^{\prime} s$ can be computed. Specifically at $\theta_{1}=90^{\circ}$ and $\theta_{2}=120^{\circ}$, the exact values of $Y^{\prime} \mathrm{s}$ are listed in row la of Table I. 
TABLE I

THE VALUES OF $\gamma_{\theta}\left(\theta_{i}\right)$ AND $\gamma_{\phi}\left(\theta_{i}\right)$ USED TO ILLUSTRATE THE CONSISTENCY PROCESS

\begin{tabular}{|c|c|c|c|c|c|c|c|c|c|}
\hline & \multicolumn{2}{|c|}{$y_{\theta}\left(90^{\circ}\right)$} & \multicolumn{2}{|c|}{$\mathrm{Y}_{\theta}\left(120^{\circ}\right)$} & \multicolumn{2}{|c|}{$\gamma_{\phi}\left(90^{\circ}\right)$} & \multicolumn{2}{|c|}{$Y_{\phi}\left(120^{0}\right)$} \\
\hline & & dB & Deg & dB & Deg & $\mathrm{dB}$ & Deg & $d B$ & Deg \\
\hline \multirow{2}{*}{1} & la & 0.601 & 31.20 & -3.340 & 16.00 & -3.820 & $-11 \cdot 30$ & $-4 \cdot 340$ & -1.20 \\
\hline & lb & 0.651 & 31.70 & -3.29 & 16.50 & -3.770 & -10.80 & -4.290 & -0.70 \\
\hline \multirow{2}{*}{2} & $2 a$ & 0.551 & 30.70 & -3.390 & 15.50 & -3.870 & -11.80 & -4.390 & $-1 \cdot 70$ \\
\hline & $2 b$ & 0.601 & 31.20 & -3.340 & 16.00 & -3.820 & -11.30 & -4.340 & -1.20 \\
\hline \multirow{2}{*}{3.} & $3 a$ & 0.576 & 30.95 & -3.365 & 15.75 & -3.845 & -11.55 & -4.365 & -1.45 \\
\hline & $3 b$ & 0.626 & 31.45 & -3.315 & 16.25 & -3.795 & -11.05 & -4.315 & -0.95 \\
\hline
\end{tabular}

$\theta_{1}=90^{\circ}, \theta_{2}=120^{\circ}, p_{0}=0.93557$

Data in row $1 \mathrm{a}$ and $2 \mathrm{~b}$ are the exact values of $\gamma^{\prime} \mathrm{s}$.

Date in row $\mathrm{lb}$ are those being shifted $+0.05 \mathrm{~dB}$ and $+0.5^{\circ}$ from the exact values.

Data in row $2 \mathrm{a}$ are those being shifted $-0.05 \mathrm{~dB}$ and $-0.5^{\circ}$ from the exact values.

Data in row 3 a are those being shifted $-0.025 \mathrm{~dB}$ and $-0.25^{\circ}$ from the exact values .

Data in row $3 b$ are those being shifted $+0.025 \mathrm{~dB}$ and $+0.25^{\circ}$ from the exact values . 
The truncation errors of the $\gamma^{\prime} \mathrm{s}$ in Eq. (15) are in general less than $0.5 \%$ of the magnitudes of $\left(a_{1}, b_{1}\right)$. In order to take these errors into consideration, we may assign to each $\gamma$ a circle of uncertainty shown in Fig. 11. That is, each complex $y$ in Eq. (17) can be anywhere in and on the circle which is centered at the exact value. The four data points 1 to 4 in Fig. 11 are assigned as representative values for each $\gamma$ which has an uncertainty circle of $0.05 \mathrm{~dB}$ or $0.6 \%$. For example, the data in row $3 a$ of each $\gamma$ in Table I correspond to point 1 in Fig. 11. Similarly, the values in row $3 b$ of each $\gamma$ correspond to point 4 in the uncertainty circle. Although values of $y$ corresponding to points 2 and 3 in Fig. 11 are not listed in Table I, row 3 represents these four values for each $\gamma$ starting from row $3 a$ and ending at row $3 b$. For illustrative purposes, two more uncertainty circles of the same size are to be considered. Let these two circles be centered at point 1 and point 4, respectively, in Fig. 11. If the reader proceeds to draw these two circles for each $y$ and designate four representative values obtained in the same way as that shown in Fig. 11, the data in rows 1 and 2 of Table I would be such that their uncertainty circles are no longer centered at their respective correct values. Namely certain systematic errors are introduced to illustrate how off-centered uncertainty circles would effect the final results . 


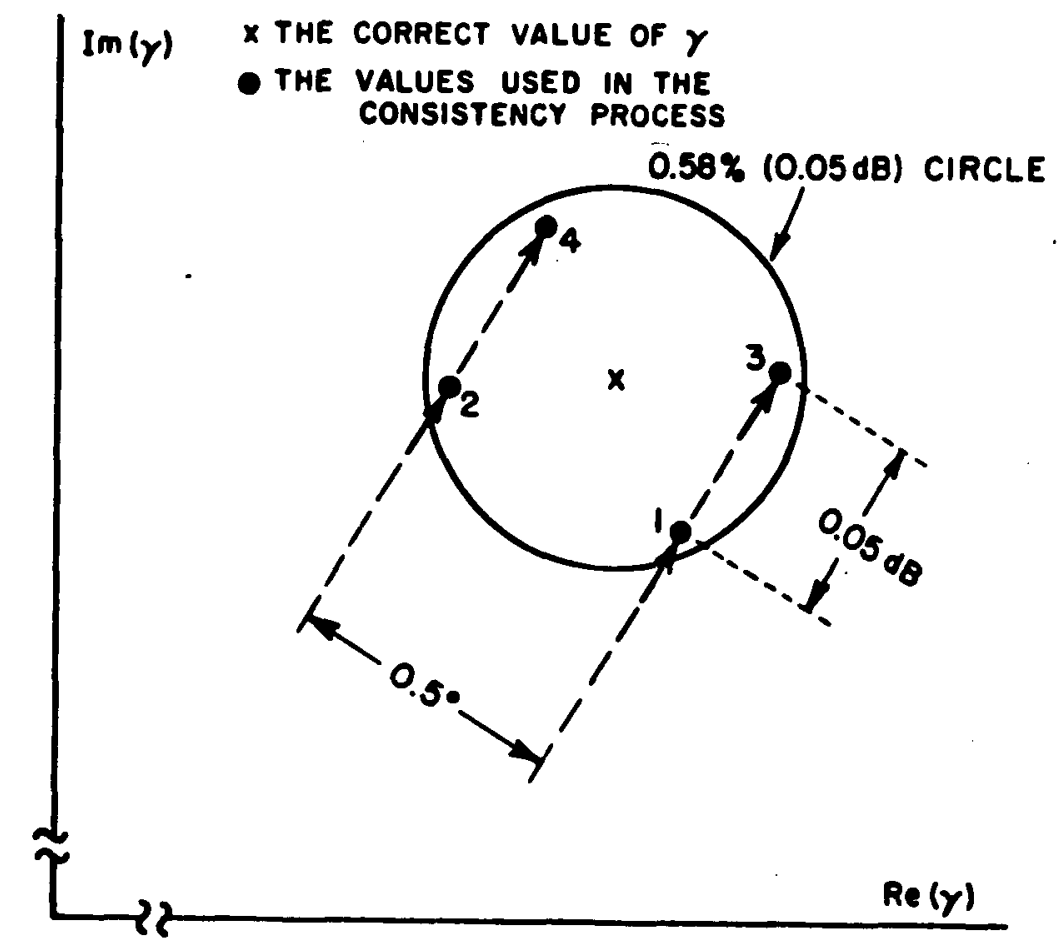

Fig. 11-.-An uncertainty circle of a $\gamma$ and its four representative data points separated by $0.05 \mathrm{~dB}$ and $0.5^{\circ}$.

B. Numerical Examples For The

Consistency Process

Before we explain the consistency process, let us first substitute the exact values of $\gamma^{\prime} \mathrm{s}$ in row $1 \mathrm{a}$ of Table I into Eq. (17) to solve for $\left(a_{1}, b_{1}\right)$ and $\left(a_{2}, b_{2}\right)$. These solutions are then used in Eq. (18) to obtain $\eta_{r}\left(a_{1}, b_{1}\right), \eta_{r}\left(a_{2}, b_{2}\right), X_{1}$ and $X_{2}$. Because the magnitudes of $\left(a_{2} b_{2}\right)$ are smaller than those of $\left(a_{1}, b_{1}\right)$, the solutions $\eta_{r}\left(a_{1}, b_{1}\right)$ and $x_{1}$ are more accurate than $\eta_{r}\left(a_{2}, b_{2}\right)$ and $X_{2}$, respectively. The value of $\eta_{r}\left(a_{1}, b_{1}\right)$ is given in row 1 of Table II. The result of $x_{1}$ is $0.056+j 0.993$ 
TABLE II SOLUTIONS ESTMATED BY USE OF THE DATA IN TABLE I

\begin{tabular}{|c|c|c|c|}
\hline & & $\eta_{r}$ & $\nu_{\mathbf{r}} P_{0}$ \\
\hline 1 & $\begin{array}{l}\text { Values from the solution using the data } \\
\text { in row la of Table I }\end{array}$ & $0.2766-j 0.1070$ & $5.02-\mathrm{j} 1.97$ \\
\hline 2 & $\begin{array}{l}\text { Estimates from consistent solutions } \\
\text { using the data in row } 1 \text { of Table I }\end{array}$ & $0.2772-j 0.1067$ & $5.31-j 2.06$ \\
\hline 3 & $\begin{array}{l}\text { Estimates from consistent solutions } \\
\text { using the data in row } 2 \text { of Table I }\end{array}$ & $0.2787-$ j 0.1044 & $5.03-\mathrm{j} 1.97$ \\
\hline 4 & $\begin{array}{l}\text { Averages of the data in row } 8 \text { and } \\
3 \text { of this Table }\end{array}$ & $0.2780-$ j 0.1056 & $5.17-j 2.015$ \\
\hline 5 & $\begin{array}{l}\text { Estimates from consistent solutions } \\
\text { using the data in row } 3 \text { of Table I }\end{array}$ & $0.2780-j 0.1057$ & $5.16-j 2.01$ \\
\hline 6 & $\begin{array}{l}\text { True values used to produce the } \gamma^{\prime} s \\
\text { in row la of Table I }\end{array}$ & $0.2783-\mathrm{j} 0.1057$ & $5.19-\mathrm{j} 2.04$ \\
\hline
\end{tabular}


whose roots can be found by use of the refractive index chart in Appendix II as $v_{x} P_{0}=3.5-j 3.4,5.02-j 1.97,8.45-j 1.84 \ldots$ --- etc. Only the first two are appropriate roots, because the others would give rise to $\varepsilon_{r}$ with a positive imaginary part. The fact that we can obtain two appropriate roots indicates nonuniqueness problems which will be discussed later. At the moment we know the true values of $\left(\eta_{Y}, v_{Y} p_{0}\right)$ as given in row 6 of Table II. Therefore, the appropriate root in question is $\nu_{\mathrm{r} P_{0}}=5.02-\mathrm{j} 1.97$ shown in row 1 of Table II. The values in rows 1 and 6 of the Table are graphically shown in Fig. 12 where all the point numbers correspond to the row numbers in Table II. While $\eta_{x}$ is seen to be accurate enough, the value of $v_{r} \rho_{0}$ falls outside of the $2 \%$-circle centered at the true value. The fact that they do not agree exactly with the true values is because there are truncation errors in Eq. (17).

Instead of simply using the exact values $\gamma^{\prime} s$ to solve for $\left(\eta_{r}, v_{r} P_{o}\right)$, we now try to let the $\gamma^{\prime} s$ in Eq. (17) be uncertain to within the $0.05 \mathrm{~dB}$ circle shown in Fig. 11. First, let us use the data in row 3 of Table I. Starting from row $3 a$, one at a time, the magnitude of each $y$ is increased by $0.05 \mathrm{~dB}$ and the phase angle of each $\mathrm{y}$ is increased by $0.5^{\circ}$.

Equations (17) and (18) are used each time in the above process. When all is done in succession, the final set of $\gamma^{\prime}$ s used is that of row $3 b$ in Table I, and a total of 256 sets of solutions can be obtained. Among these 256 sets of solutions we first exclude those chose $\eta_{r}\left(a_{2}, b_{2}\right)$ deviates 

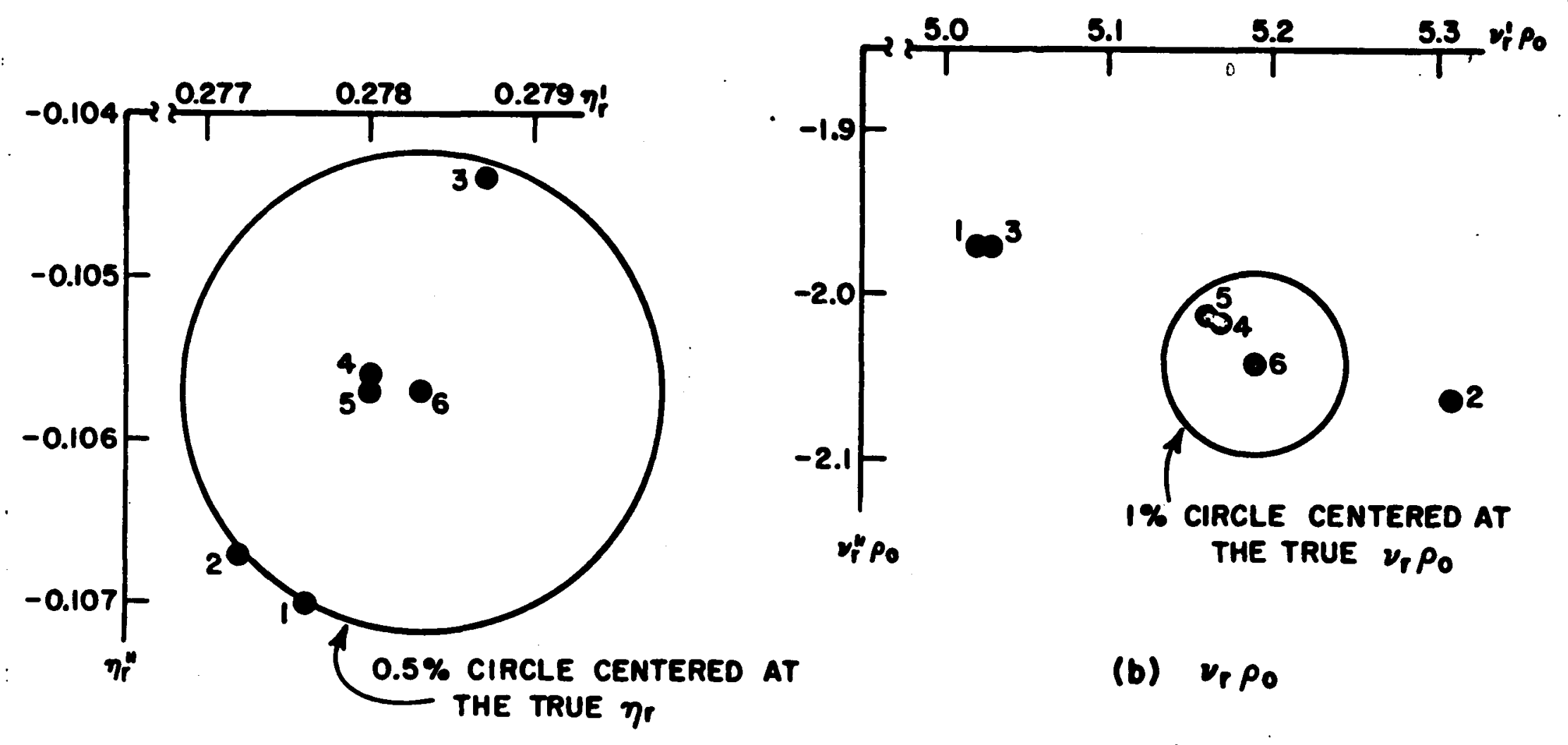

(a) $\eta_{r}$

Fig. 12--Complex-plane representation of the data in Table II. 
from $\eta_{r}\left(a_{1}, b_{1}\right)$ by more than $20 \%$ in magnitude. The remaining solutions $X_{1}$ and $X_{2}$ are then used to solve for an approximate root $\left(v_{r} \rho_{0}\right)$ by the formula

$$
\left(v_{r} \rho_{0}\right)_{a}=\frac{-\left(X_{1}-X_{2}\right)+\sqrt{\left(X_{1}-X_{2}\right)^{2}+4\left(1+X_{1} X_{2}\right)}}{1+X_{1} X_{2}}
$$

which is obtained by use of the recurrence formula (7c). Each value of $\left(\nu_{r} \rho_{0}\right)_{2}$ is compared with $\nu_{r} \rho_{0}$ obtained from the corresponding $X_{1}$ by use of the refractive index chart. Those solutions whose $\left(v_{r} P_{0}\right)_{a}$ deviates from $v_{x} \rho_{0}$ by more than $25 \%$ in magnitude are again excluded. The remaining solutions are then finally considered as consistent solutions which give the results shown in Figs. 13 and 14. The estimated values in these figures are the arithmetic means of the data points shown. They are listed in row 5 of Table II and are shown in data points 5 of Fig. 12. It is evident that these estimated values of $\left(\eta_{x}, \nu_{Y} P_{0}\right)$ obtained by the consistency process are in better agreement with the true values shown in data points 6 of Fig. 12 .

We next employ the consistency process to the data in row 1 of Table 1 . Starting from row $l a$, one at a time, the magnitude and phase angle of each $y$ are increased by $+0.05 \mathrm{~dB}$ and $+0.5^{\circ}$, respectively. Successively, 256 sets of solutions are obtained by use of Eq. (17) and these solutions are then used in Eq. (18). After application of the consistency criteria mentioned above, we obtain the final results in Figs. 15 and 16. Similarly, Figs. 17 and 18 show the results 


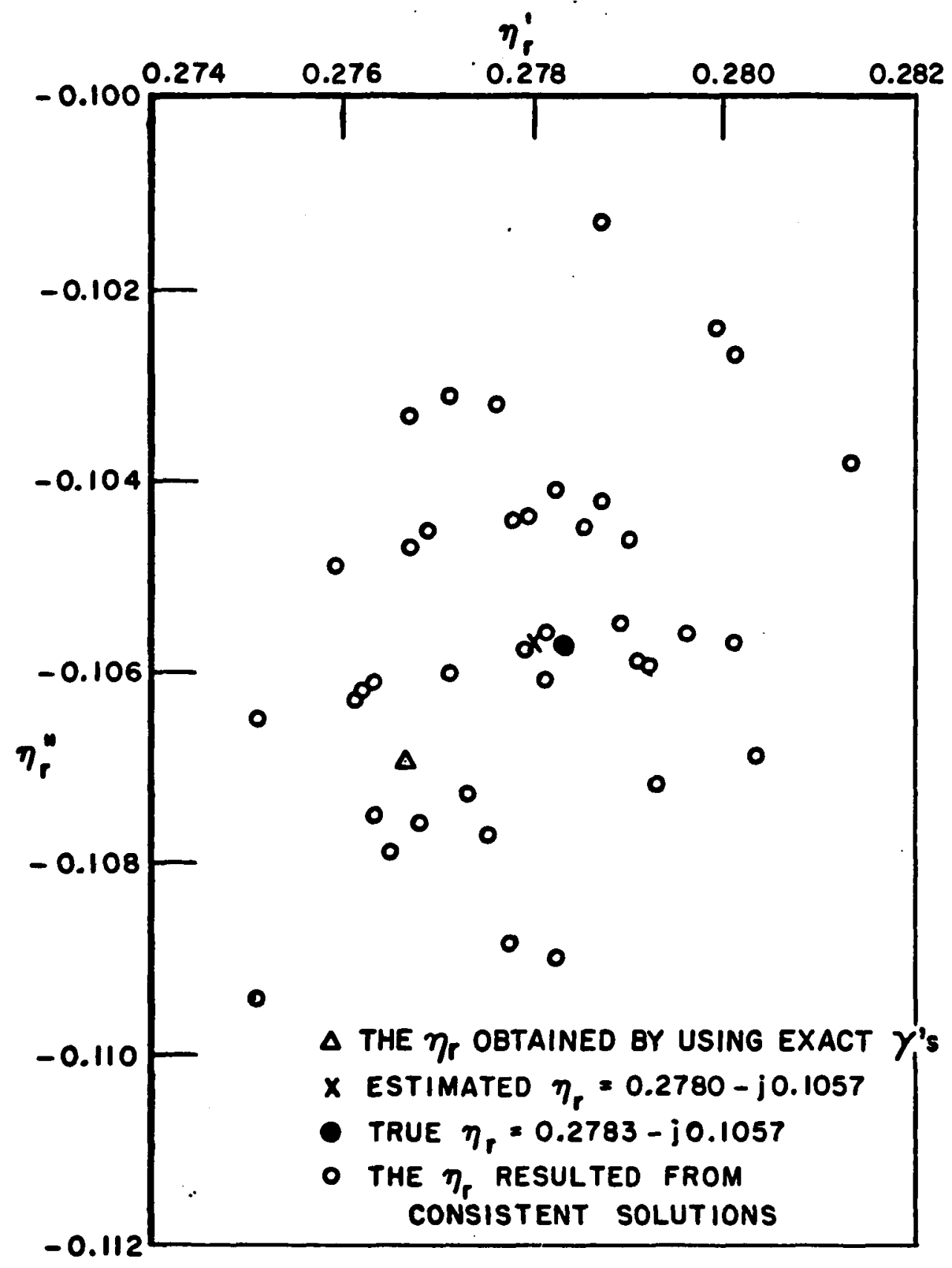

Fig. 13--Distribution of $\eta_{x}$ from consistent solutions produced by use of the data in row 3 of Table I. 


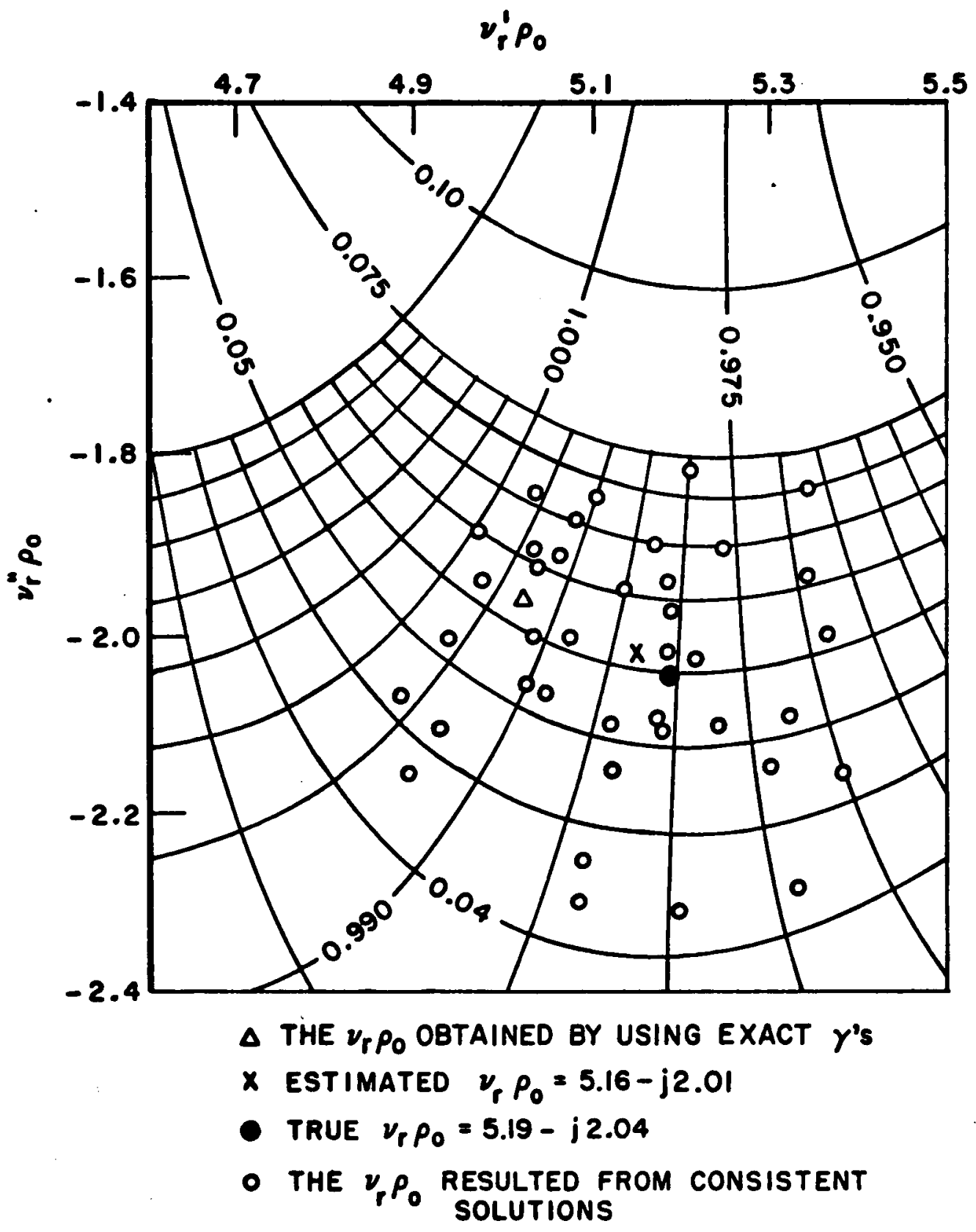

Fig. 14--Distribution of $v_{\mathbf{r}} \mathrm{p}_{\mathrm{O}}$ from consistent solutions produced by use of the data in row 3 of Table I. 


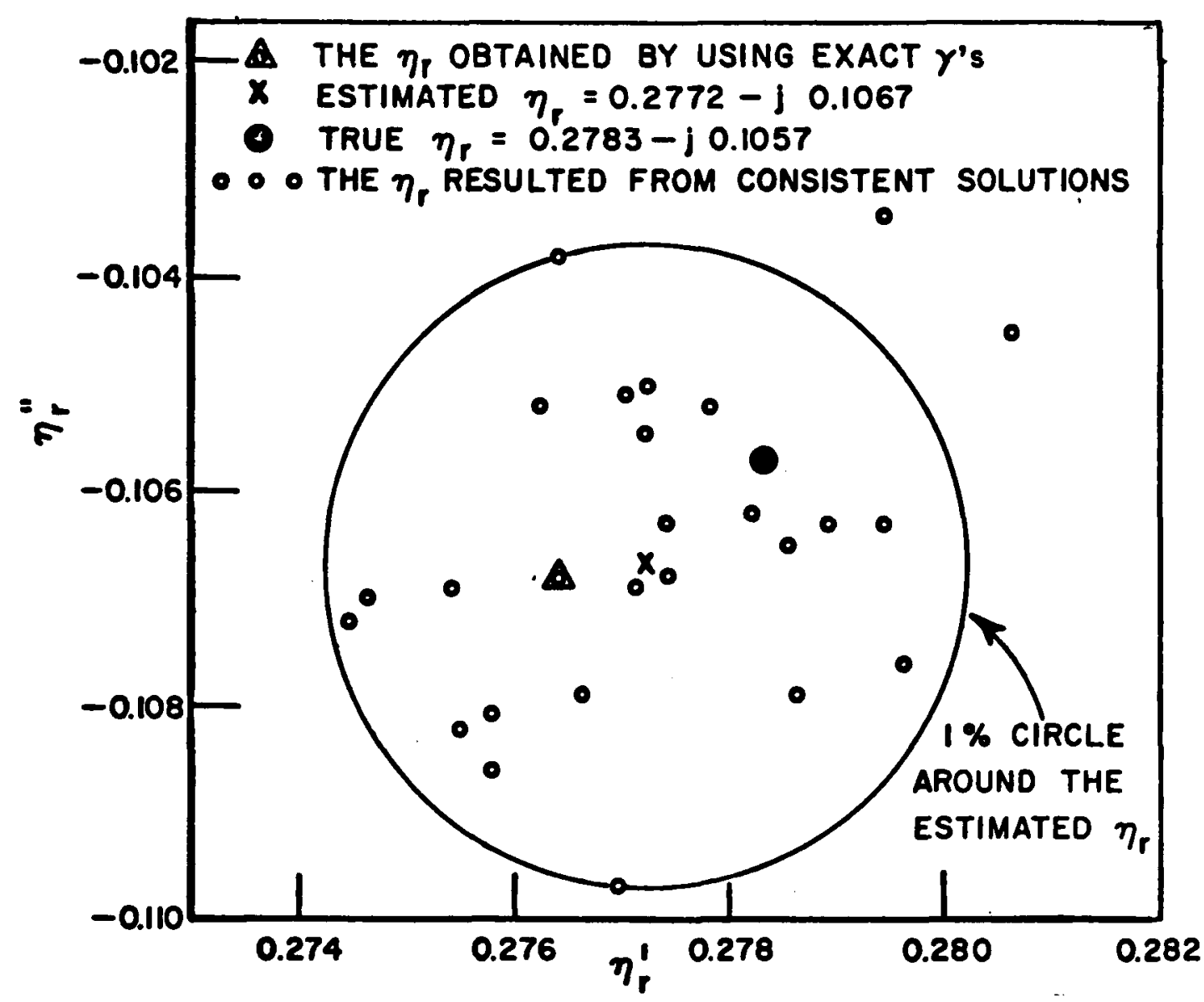

Fig - 15--Distribution of $\eta_{r}$ from consistent solutions produced by use of the data in row 1

of Table I.

produced by use of the data in row 2 of Table I. Comparisons of Figs. 15 and 16 with Figs. 17 and 18 indicate that their distribution of data points are concentrated on opposite sides of the true values. The reason for this is because the uncertainty circles associated with row 1 data in Table I are centered at points on one side of the correct $\gamma^{\prime} s$ while the uncertainty circles assoicated with row 2 data of Table I are centered on the opposite side. The estimated values in Figs. 15 to 18 


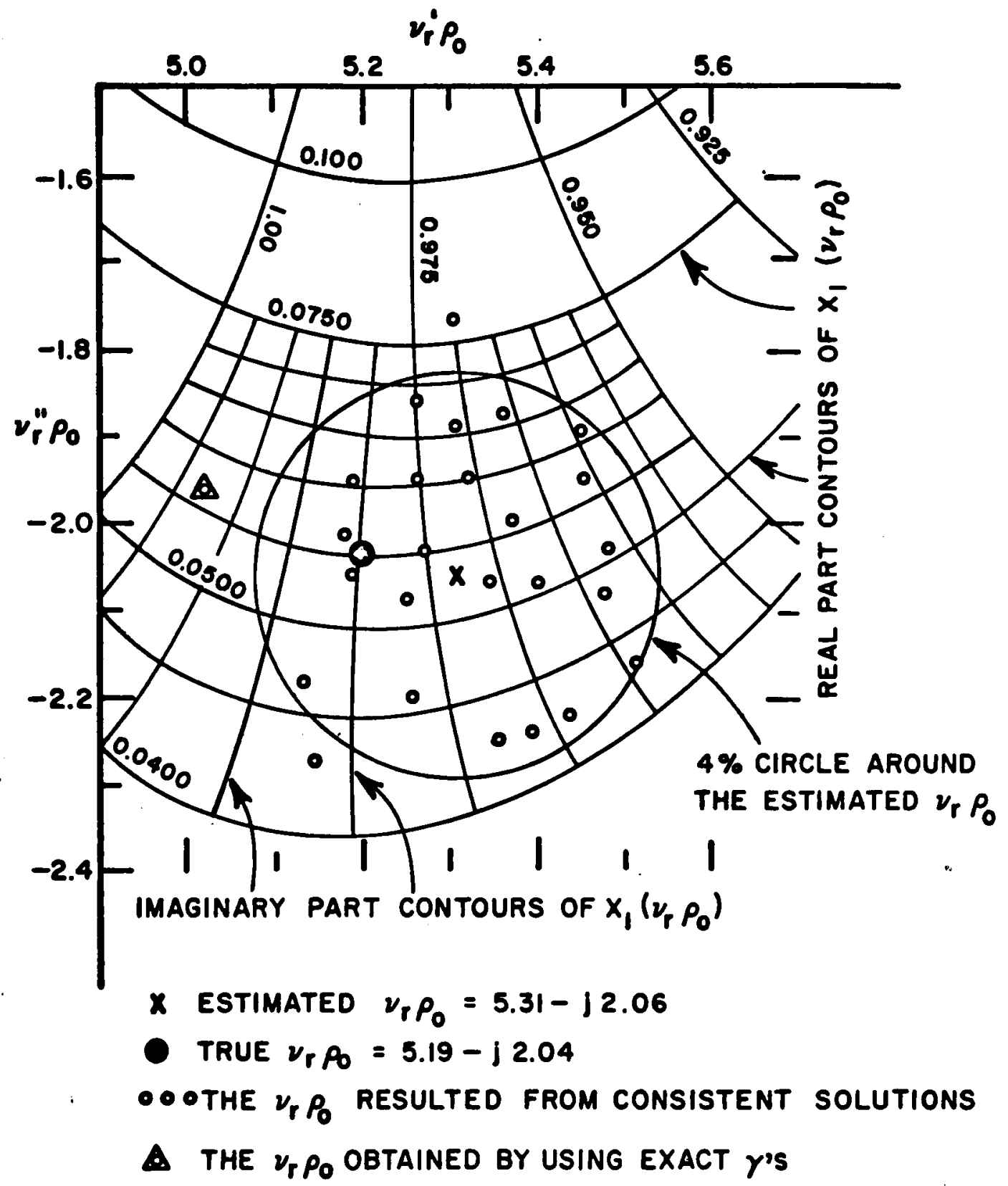

Fig. 16--Distribution of $\nu_{r} \rho_{0}$ corresponding to Fig - 15 . 


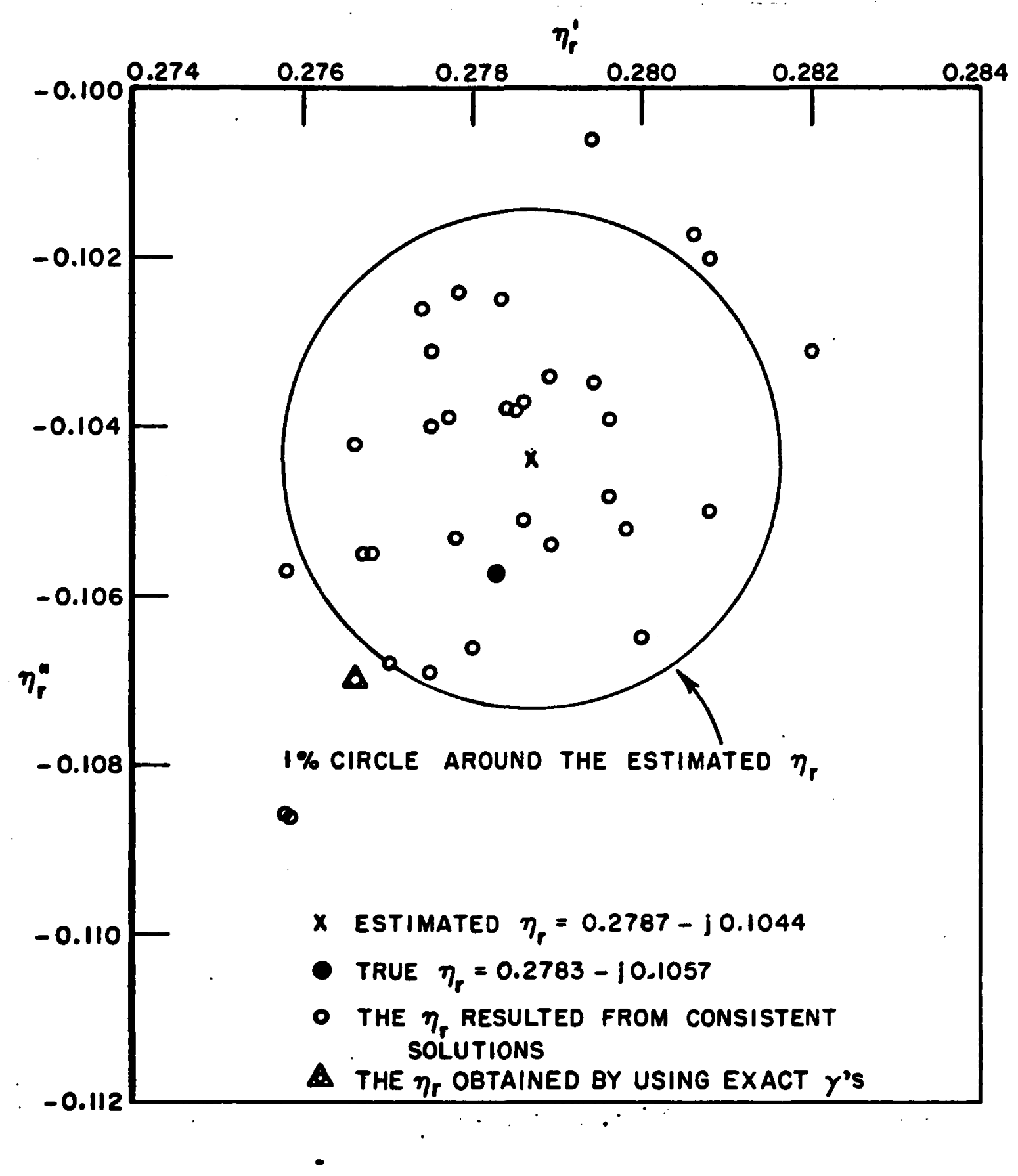

Fig. 17--Distribution of $\eta_{\boldsymbol{r}}$ from consistent solutions by use of the data in row 2 of Table $I$. 


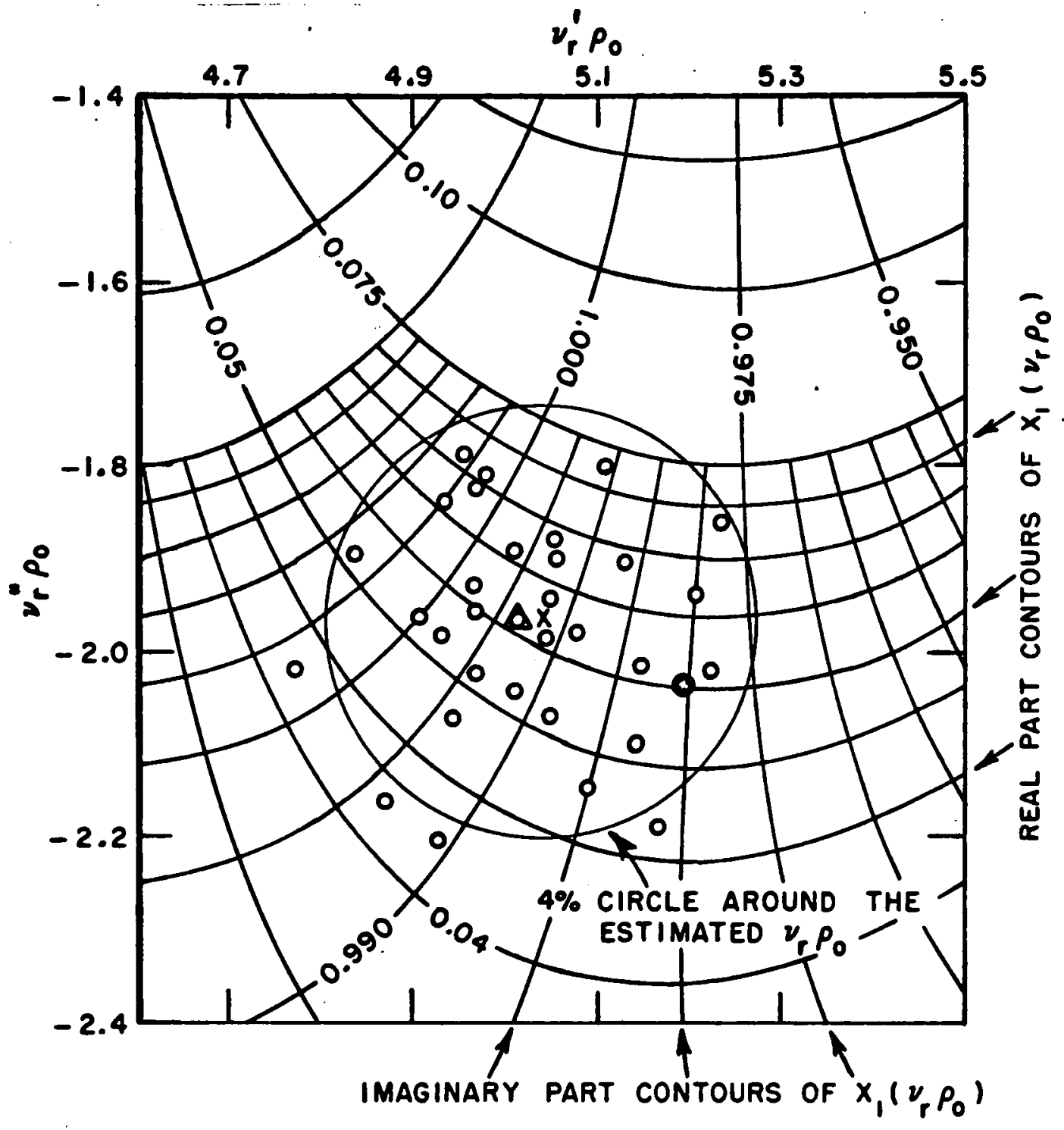

$x$ ESTIMATED $\nu_{p} p_{0}=5.03-11.97$

TRUE $\nu_{P} P_{0}=5.19-j 2.04$

- THE $\nu_{P} P_{0}$ RESULTED FROM CONSISTENT SOLUTIONS

A THE $\nu_{\text {r }}$ OBTAINED BY USING EXACT $\gamma^{\prime}$ 's $\because \ldots$.

Fig. 18--Distribution of $v_{r} \rho_{0}$ corresponding to Fig. 17 . 
are listed in rows 2 and 3 of Table II. The values in row 4 are simply the averages which are shown in data points 4 of Fig. 12. As can be expected, the data points 4 are closely in agreement with the data points 5 in Fig. 12. This is a good indication that the consistency criteria used in these numerical illustrations are valid and useful in improving the accuracy with which $\left(\eta_{\mathbf{r}}, v_{\mathrm{r}} \rho_{0}\right)$ can be solved using Eq. (17) in which only approximate values of $y^{\prime} s$ are used.

It may be interesting to consider another example using a different sphere. Let us choose a sphere of parameters $\left(\rho_{O}, \eta_{r}, v_{r} \rho_{O}\right)=(0.93557$, $0.3979-j 0.0641,4.1-j 1.1)$. The normalized principal-plane bistatic fields are obtained at bistatic angles $\theta_{1}=100^{\circ}$ and $\theta_{2}=130^{\circ}$. The uncertainty circle for each $y$ is of $0.05 \mathrm{~dB}$ and centered at the true $\gamma$ as shown in Fig. 11. Using the same consistency criteria used in the previous example gives rise to similar results for the estimated values of $\left(\eta_{r}, v_{r} p_{0}\right)$. That is, thery agree with the true values to better than 1\%. We show in Figs. 19 and 20 the distributions of the coefficients $\left(a_{1}, b_{1}\right)$ and $\left(a_{2}, b_{2}\right)$ obtained by the consistency process. One can see that the strengths of $\left(a_{1}, b_{1}\right)$ are greater than those of $\left(a_{2}, b_{2}\right)$ by an order of magnitude. While the data points of $\left(a_{1}, b_{1}\right)$ are distributed about $1 \%$-circles, the distributions of $\left(a_{2}, b_{2}\right)$ are about $10 \%$-circles.

Before we close this chapter we note that there is an alternative criteria for the consistency of $\nu_{r} \rho_{O}$. If one uses the value of $\left(\nu_{r} \rho_{0}\right)_{a}$ obtained in Eq. (20) as the argument of Eq. (7c), then $X_{1}$ can be 


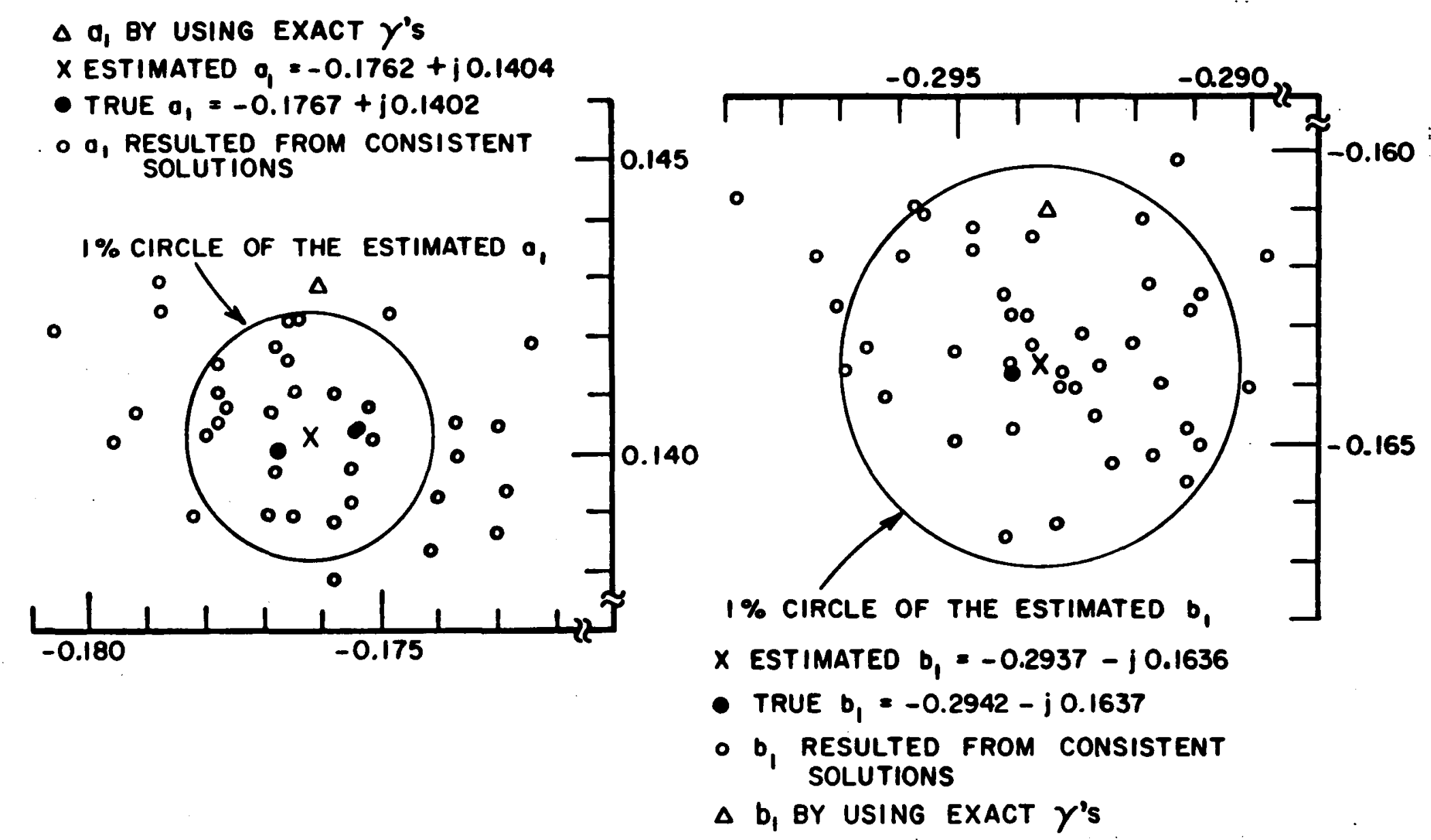

Fig. 19-- Distributions of dipole coefficients $\left(a_{1}, b_{1}\right)$ obtained from the consistent process. 


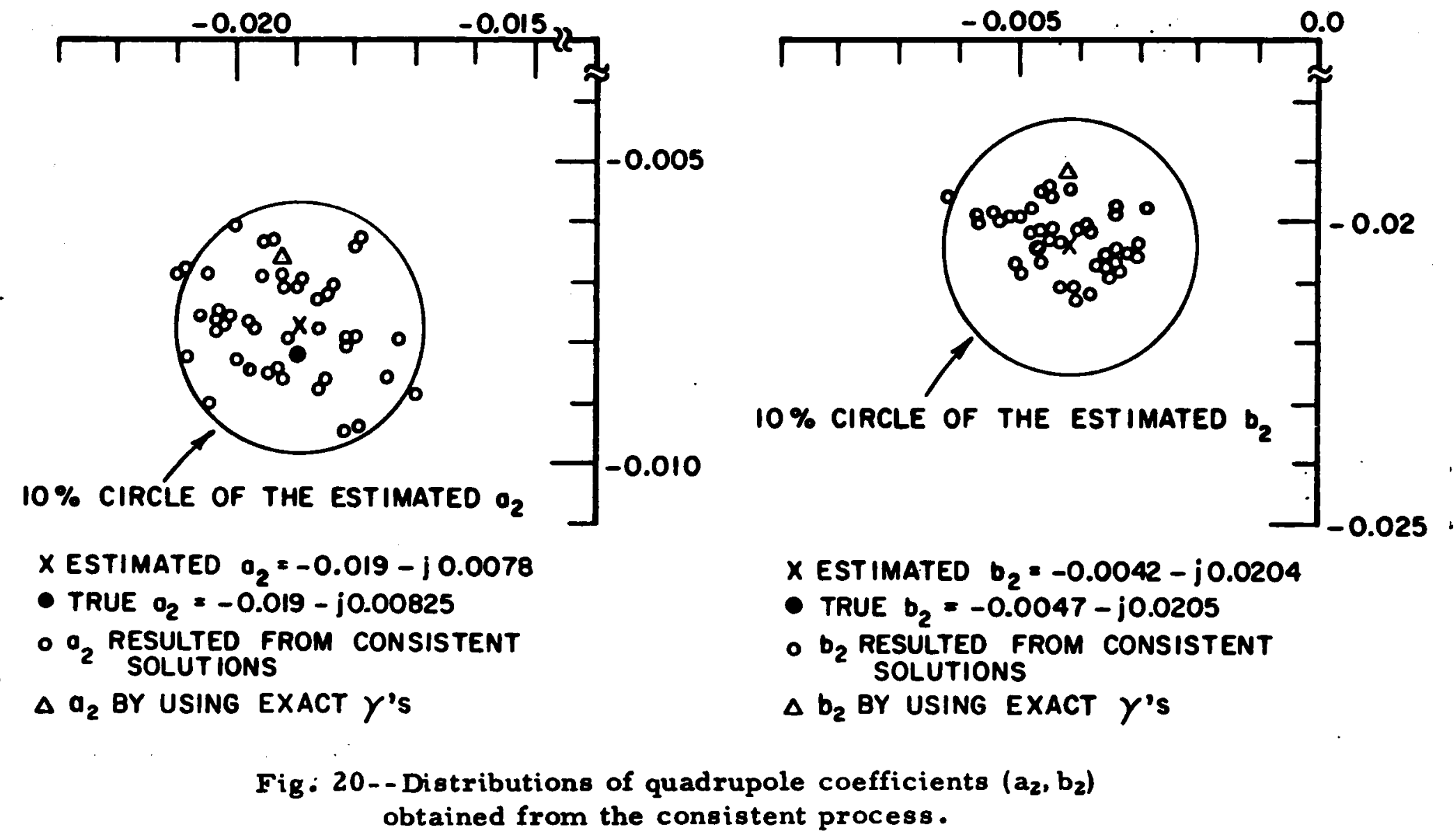


approximately calculated. This $X_{1}$ can then be compared with the $X_{1}$ obtained by $\mathrm{Eq} \cdot(18 \mathrm{~b})$. An agreement to within $25 \%$ is also found to be a good criterion by numerical examples. Also important to note is that when the uncertainty circle of each $\gamma$ is allowed to be bigger than that of a $0.05 \mathrm{~dB}$-circle, the results of the consistency process would have a smaller number of consistent solutions for the same number of total solutions. In one particular example, more than one hundred consistent solutions are found from the total of 256 when $0.05 \mathrm{~dB}-$ circle of uncertainty is used for each $\gamma$. The same example yields only twenty consistent solutions when an uncertainty circle of $0.1 \mathrm{~dB}$ is assigned to each $\gamma$. Finally, we note that a computer program developed Richmond[14] using Crout[15] method has been used to solve Eq. (17).

\section{The Consistency Process Summarized}

We now conclude this chapter by summarizing the consistency process as follows:

1) Uniformly assign to each $\gamma$ in Eq. (17) an uncertainty circle of $0.05 \mathrm{~dB}$ and select four representative values in the circle, as illustrated in Fig. 11.

2) Solve for 256 sets of coefficients $\left(a_{1}, b_{1}\right)$ and $\left(a_{2}, b_{2}\right)$ from Eq. (17) by using the four representative values in succession for each of the four $\gamma^{\prime} s$. 
3) Obtain $\eta_{r}\left(a_{1}, b_{1}\right), \eta_{r}\left(a_{2}, b_{2}\right), x_{1}$ and $X_{2}$ from Eq. (18) by use of the 256 solutions for $\left(a_{1}, b_{1}\right)$ and $\left(a_{2}, b_{2}\right)$.

4) Exclude those solutions whose $\eta_{r}\left(a_{2}, b_{2}\right)$ deviates from the corresponding $\eta_{r}\left(a_{1}, b_{1}\right)$ by more than $20 \%$.

5) Exclude those solutions, remained from step 4, whose value of $\left(v_{\mathrm{r}} \mathrm{P}_{\mathrm{O}}\right)_{\mathrm{a}}$ by Eq. (19) deviates from the root $v_{\mathrm{r}} \mathrm{po}_{0}$ of the corresponding $X_{1}$ by more than $25 \%$.

6) Consider the remaining sets of coefficients as consistent solutions, and estimate their $\eta_{r}\left(a_{1}, b_{1}\right)$ and $v_{x} p_{0}$ of $X_{1}$ as illustrated in Figs. 13 and 14.

7) Obtain the desired parameters $\mu_{Y}=\eta_{Y} \nu_{I}$ and $\epsilon_{e}=\nu_{Y} / \eta_{Y}$. The consistency criteria imposed here are somewhat flexible and dependent of the extent of errors involved in the system of Eq. (17). Our examples have shown that they indeed give solutions to the parameters $\left(\mu_{r}, e_{r}\right)$ with less than $1 \%$ deviation from the true values, provided the true values of each $y$ are known. However, from the standpoint of obtaining a set of accurate parameters, these criteria may be relaxed or made more strict when errors in Eq. (17) become smaller or larger. Strictly speaking, consistency criteria may differ from one specimen to another if the same accuracy is desired. As a consequence, it is possible in some particular cases that our consistency criteria are insufficient to produce accurate results for $\left(\eta_{\mathbf{r}}, v_{\mathbf{r}} \rho_{\mathrm{O}}\right)$. 


\section{CHAPTER V \\ EXPERIMENTATIONS AND FINAL RESULTS}

This chapter describes the experimental aspects of obtaining the normalized bistatic fields. Two frequencies, 6.17 and $7.03 \mathrm{GHz}$, are used in our measurements. All spheres used are the same diameter $d=1.27 \mathrm{~cm}$. Specimens [8] A, I, K and L lobtained from Emerson and Cuming Inc.) are chosen for our scattering method. Scattered field components at $\theta=90^{\circ}, 120^{\circ}$ and $135^{\circ}$ are measured. Results of each specimen sphere are then normalized by those corresponding results of a conducting sphere to obtain the $\gamma^{\prime}$ s defined in Eq. (16).

A. Transmitter And Receiver Systems

Measurements to be made in the two principal planes are shown in Figs. 21 and 22. A frequency-stablized klystron oscillator is connected to a corrugated horn of low sidelobes. [16] On the symmetry axis of the horn a sphere is placed in the far-zone of the transmitting antenna.

Two hog horns [17] have been specially designed for E-plane and $\mathrm{H}$-plane receptions. Both horn antenna have the same aperture dimensions as shown in Fig. 23. However, they have different 


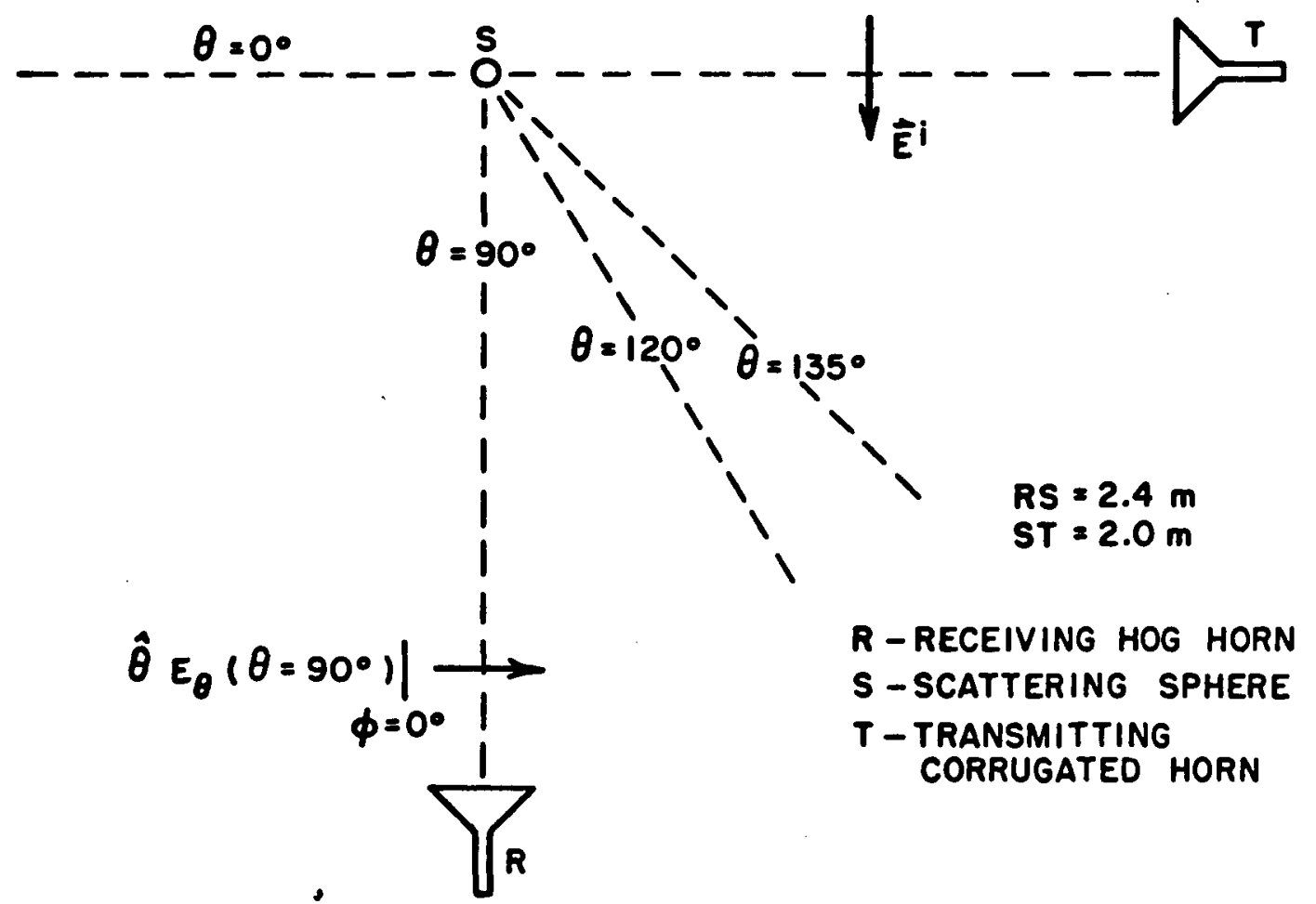

Fig. 21. Geometry for the E-plane measurements at 6 and $7 \mathrm{GHz}$.

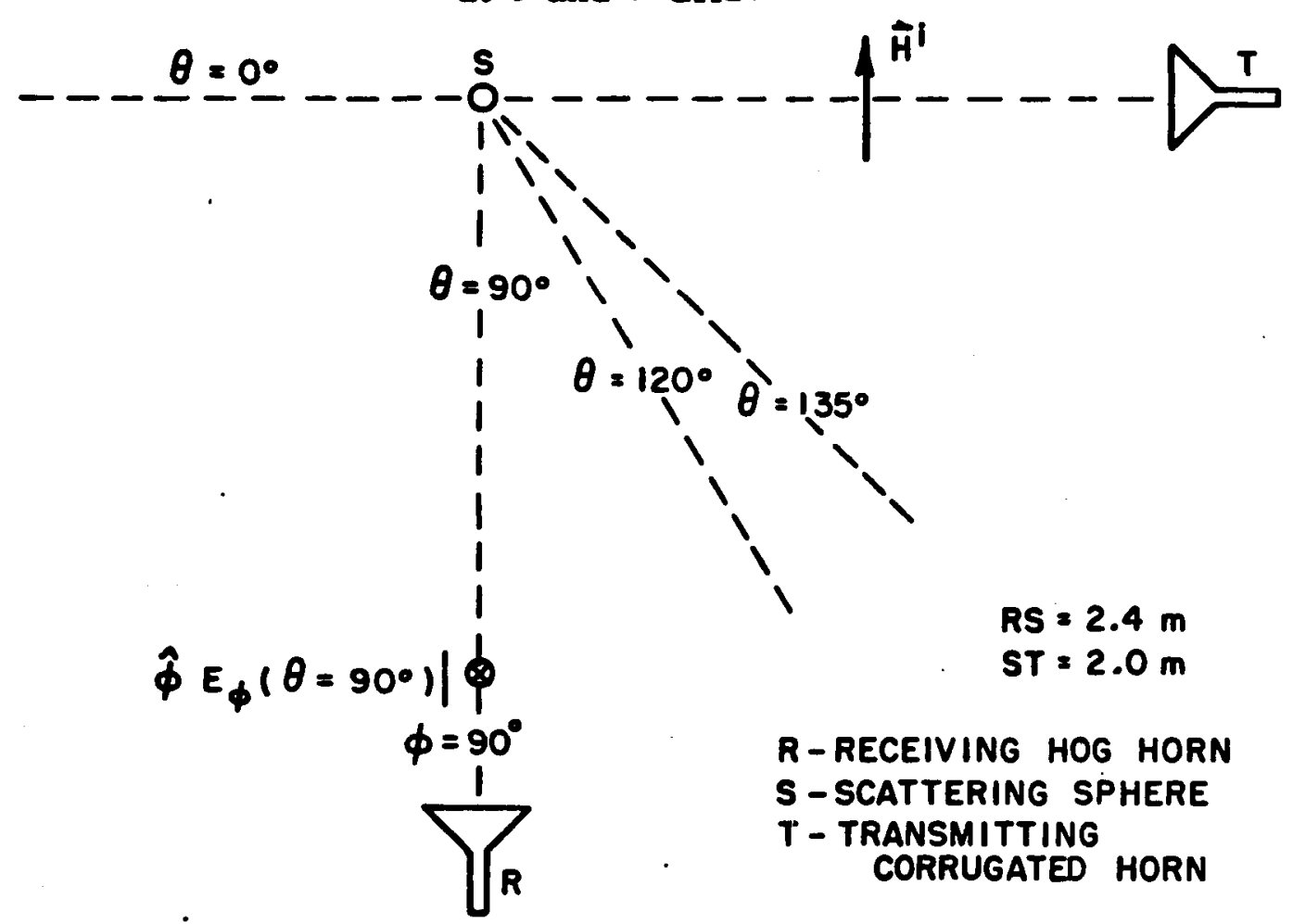

Fig. 22--Geometry for the H-plane measurements at 6 and $7 \mathrm{GHz}$. 


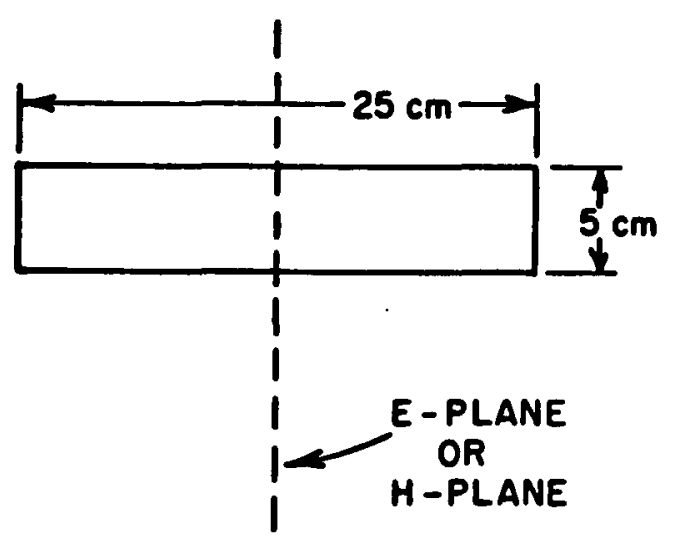

Fig. 23--Aperture dimensions of the two receiving hog horns.

polarizations. For example, the hog horn used in the H-plane has the scattered electric field component $E_{\phi}$ parallel to its longer side of the aperture. A receiving hog horn is placed in the far-zone of the scattering sphere. To the receiving horn we connect a Phase/ Amplitude Wide Range Receiver (Series 1650 of Scientific Atlanta, Inc.). Both amplitude and phase of the received scattered field are then recorded by a Rectangular Recorder (Series 1540 of Scientific Atlanta, Inc.).

B. Scattering Range And Target Support

The distances ST and RS indicated in Figs. 21 and 22 are chosen such that far-zone criteria for the antennas at 6 and $7 \mathrm{GHz}$ are 8 atisfied. The width $5 \mathrm{~cm}$ of each hog horn aperture corresponds to a chord of $1^{\bullet}$ in bistatic angle $\theta$. The three bistatic angles are chosen primarily on the consideration that no excessive power would be directly coupled from the transmitter to the receiver. In the absence of a target sphere the receiver can thus be nulled with relative ease to approximate our scattering range as a free space. 
Our target sphere is supported by a square styrofoam plate as shown in Fig. 24. The plate has a thickness of about $0.4 \mathrm{~cm}$ and is dented at the center by pressing down a sphere of $1.27 \mathrm{~cm}$ in diameter. Depth of the dent is about $0.2 \mathrm{~cm}$. The styrofoam plate is fastened to four dacron strings through its four corners. These four strings are then tied together on the top to the ceiling of a room housing our scattering range. At the bottom, the four strings are tied to a pedestal which can be made to rotate $360^{\circ}$ horizontally. Initial adjustments are made to the styrofoam plate such that our target sphere is correctly positioned. When the pedestal of the support is rotated $360^{\circ}$ the motion of the center of correctly positioned sphere is then minimized.

\section{Measurement Procedure}

Measurement procedures for each bistatic angle $\theta$ are essentially the same, although results may vary. We shall use a set of measurements for $7.03 \mathrm{GHz}$ at $\theta=120^{\circ}$ in the $\mathrm{H}$-plane as an illustration. The hog horn of correct polarization is first placed at $\theta=120^{\circ}$ as shown in Fig. 22. When the overall transmitter systems, receiver systems and scattering environments have reached a steady state, the receiver unit is then nulled in the absence of any target sphere. At the present stage of investigation, nulling is made only at a particular orientation of the target support. When the whole support shown in Fig. 24 is rotated $360^{\circ}$, we record in Fig. 25 the background noises which are 


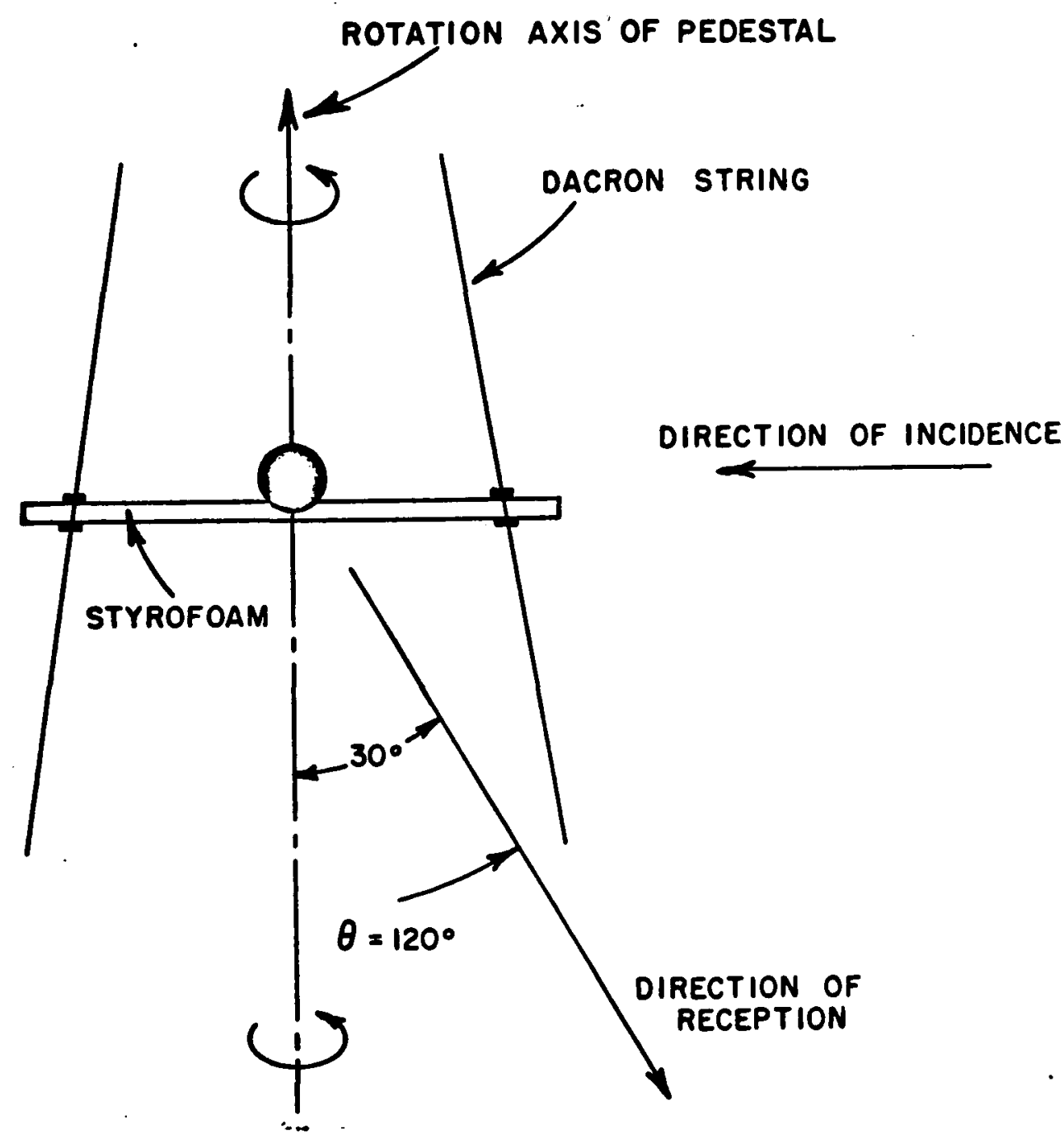

Fig. 24--A side view of the dented styrofoam plate used to support the target sphere (the plate can be rotated horizontally $\left.360^{\circ}\right)$.

the combined scattered field amplitudes from the styrofoam plate and the four dacron strings. There are four nulled zones designated by I to IV in the figure. It is in these four zones that our scattering range is properly nulled.

By holding the nulled conditions, a conducting sphere of $1.27 \mathrm{~cm}$ in diameter is next placed on the target support which is now being rotated in reverse direction $360^{\circ}$. Both amplitude and phase angle 

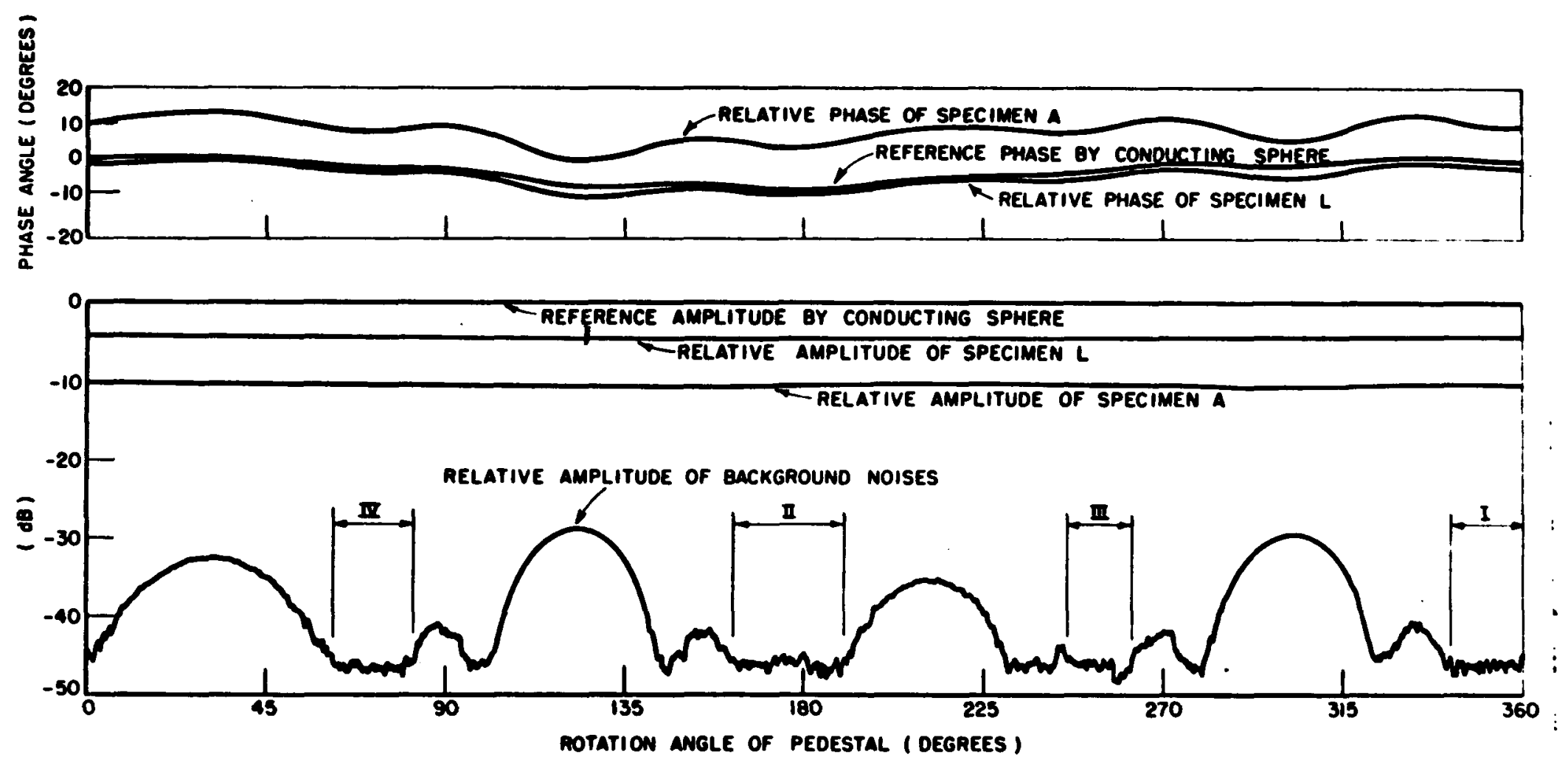

Fig - 25--The H-plane measurements at $\theta=120^{\circ}$ made for specimens $A$ and $L$ at $7.03 \mathrm{GHz}$. 
of its scattered field are recorded as shown in Fig. 25. We use this recorded amplitude as a reference level and note that background noises are relatively smaller than $-45 \mathrm{~dB}$ in the four nulled regions. In the off-nulled regions, the strongest background level is about $-30 \mathrm{~dB}$ as compared to the reference level. It is thus natural to see the amplitude pattern recorded for the reference sphere is essentially constant in the entire $360^{\circ}$ region. The corresponding phase pattern, on the other hand, is far from being constant. In addition to irregularities which can be identified with the background pattern, there is a complete period of variation that $c$ an be identified with rotation of our target support structure. The latter is of significant importance, because it describes motion of the target sphere during rotation of the pedestal. For instance, the recorded phase of the reference sphere is set zero in region I but becomes about $-8^{\circ}$ in region II after a rotation of $180^{\circ}$ in the pedestal's rotation angle. This observation simply means that the target sphere has been carried farther away from the receiving horn during the rotation. Characteristics of target motion during a rotation of its support depend on relative position of the target and the rotation axis of the support. However, for the same target support, different spheres of the same size should have the same path of motion, provided they are placed into the dent of the styrofoam plate exactly in the same manner. Therefore, a homogeneous 
and isotropic specimen would exhibit the same phase pattern as that of the conducting sphere.

By still holding the same nulled conditions, specimens $A$ and $L$ are successively measured and recorded in Fig. 25. Both specimens scatter less power than the conducting sphere at this particular bistatic angle in the H-plane. Consequently, their measured amplitudes and phase angles are less accurate than those of the conducting shpere. In the nulled regions of the figure, for examples, the inaccuracy involved in the field amplitude of the conducting sphere can be as small as $0.5 \%$ while that of specimen $A$ can be as large as $2 \%$. The corresponding inaccuracies in phase angles are implicit in the definition of a complex number, provided background noises in these regions are truly random in nature.

The procedures described above for measuring specimens $A$ and I take about five minutes if the null is maintained. After completion of the measurements in Fig. 25, the sphere target is removed from the support and the receiver systems are renulled. We then repeat the above procedures all over and obtain another set of measurements similar to Fig. 25. These two sets of data may be considered independent in both time and space, because not only the receiver systems are renulled at different times but the orinetations of specimen spheres are arbitrary when they are being placed in the support. 


\section{Measurement Uncertainties}

The background noise in Fig. 25 represents one kind of measurement uncertainty. It is indicated in the four nulled regions that the null level is about $-45 \mathrm{~dB}$ compared to the field strength scattered by the conducting sphere. Therefore, the uncertainties associated with the conducting sphere measurements are about $0.05 \mathrm{~dB}$ and $0.5^{\circ}$, respectively, in amplitude and phase angle. On the other hand, the uncertainties for specimen $A$ are about $0.15 \mathrm{~dB}$ in amplitude and $1.5^{\circ}$ in phase angle, because the noise level is only $-35 \mathrm{~dB}$ compared to the signal power. Therefore, it is important to note that uncertainties of measured data for each specimen measured at each bistatic angle in each principal plane need be assessed individually. In general the lower the null level the less uncertainties are involved in measured data.

Our specimen spheres and the conducting sphere are gauged by a micrometer to have $1.27 \mathrm{~cm}$ in diameter with an uncertainty of less than $0.5 \%$. The frequency of our klystron oscillator is read from a wavemeter to the third digit. The coordinate angle $\theta$ is determined by gauging distances separated by two specific points. The uncertainty of placing a receiving horn at $\theta=90^{\circ}, 120^{\circ}$ and $135^{\circ}$ is estimated to be less than $0.5^{\circ}$. All the imprecisions mentioned here could cause errors in the measured data shown in Fig. 25. By use of Eq. (13) we show in Figs. 26 and 27 the maximum possible errors that could occur 


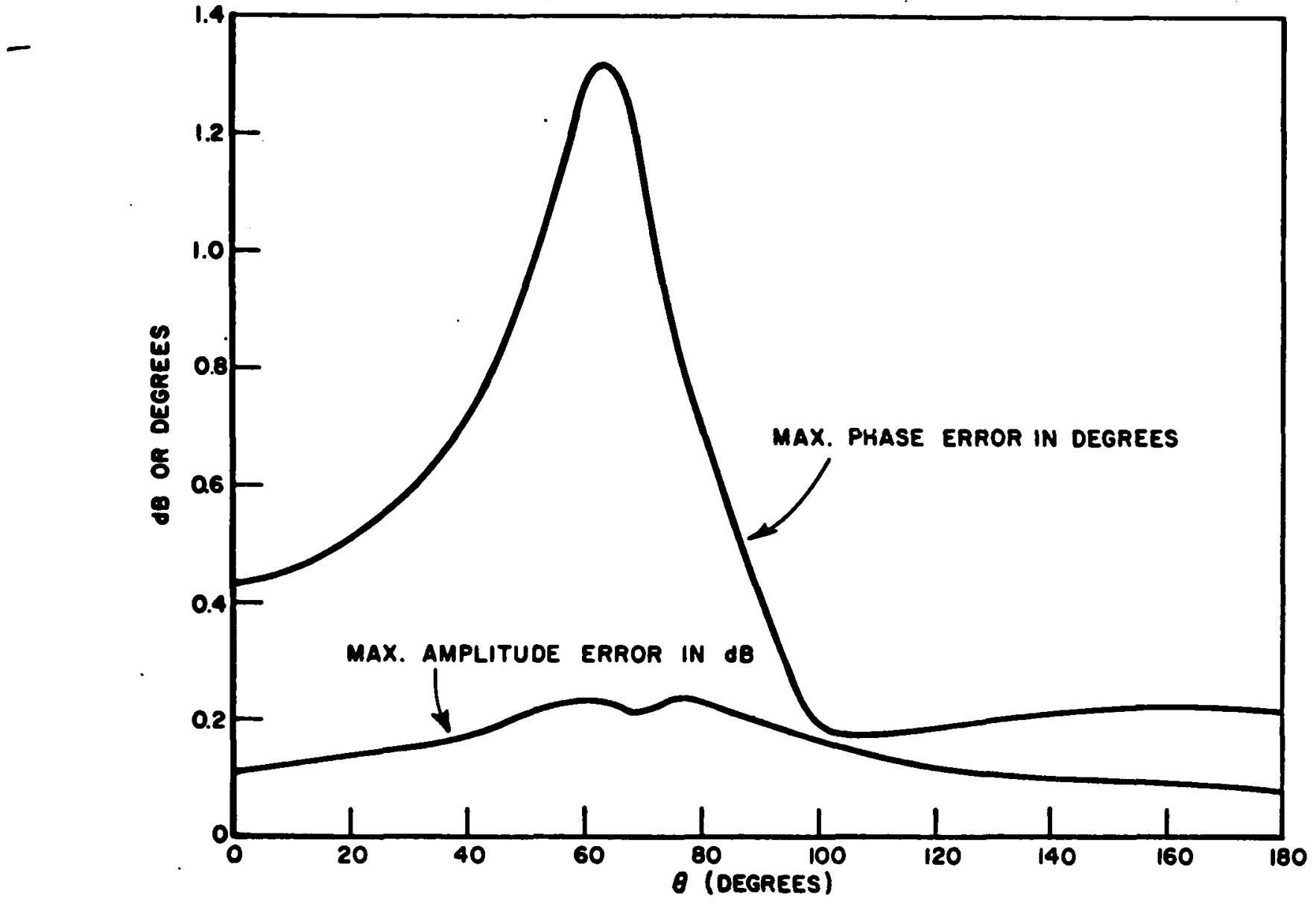

Fig. 26--Maximum possible amplitude and phase errors in the E-plane of a conducting sphere of $p_{0}=\pi d / \lambda_{0}=0.9$, when $p_{0}$ is allowed to change $\pm 0.5 \%$ and $\theta$ is allowed to deviate $\pm 0.5^{\circ}$.

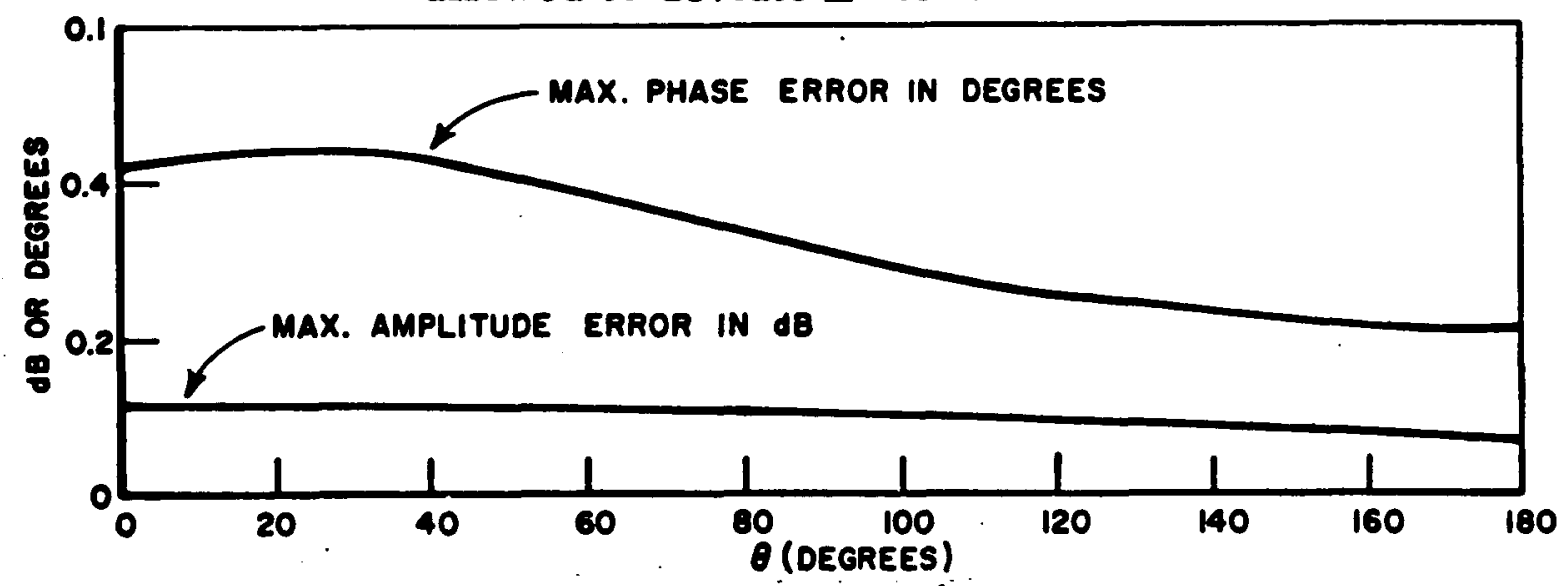

Fig. 27--Maximum possible amplitude and phase errors in the $H$-plane of a conducting sphere of $p_{0}=\pi d / \lambda_{0}=0.9$, when $p_{0}$ is allowed to change $\pm 0.5 \%$ and $\theta$ is allowed to deviate $\pm 0.5^{\circ}$. 
to the scattered field components of a conducting sphere of $p_{0}=\pi d / \lambda_{0}$ $=0.9$. The same order of errors could occur to measured data of our specimens sphere, simply because we always have imprecision errors due to mechanical gaugings .

The imprecision errors in electrical detection of scattered field components are given by the manufacturer's specifications as $0.5 \mathrm{~dB}$ over $40 \mathrm{~dB}$ dynamic range and $5^{\circ}$ over $\pm 180^{\circ}$ scales in a period of thirty minutes stability.

E. Homogeneity And Isotropicity

An inhomogeneous and/or anisotropic specimen sphere should exhibit certain dependence of its scattered field components (at a fixed $\theta$ ) on orientation of the sphere. Take the recorded amplitude and phase angle for specimen $L$ in Fig. 25 for instance. Both have almost identical characteristics as those recorded for the conducting sphere, when the target support is rotated $360^{\circ}$. In other words, there is no noticeable dependence of the field component on the orientation of specimen $L$. This conclusion is confirmed also by different positionings at different bistatic angles. Consequently, specimen L can be safely assumed homogeneous and isotropic. As for specimen A, more irregularities are noticeable in comparisons with the conducting sphere. However, these irregularities are all within the uncertainties associated with the measured data of specimen A. For 
example, the relative phase angle of specimen $A$ has less than $2^{\bullet}$ variations in the four nulled zones of Fig. 25. Therefore, we also consider specimen $A$ as an isotropic and homogeneous sphere. In fact, all the specimens (A, I, $K$ and $L[8]$ ) reported here are found to be homogeneous and isotropic.

A specimen sphere is not to be assumed homogeneous and isotropic if its orientational characteristics are greatly different from those of a conducting sphere. The extent of difference is considered great if they exceed the uncertainties induced by noise levels of a particular instance.

F. Data Reduction

Data reduction for each set of measurements is the same. We use Fig. 25 and its independently repeated measurements as illustrations. In Fig. 25, the phase angle of specimen A relative to that of the conducting sphere is $10^{\circ}, 12^{\circ}, 12.5^{\circ}$ and $10.5^{\circ}$, respectively, in the nulled regions I, II, III and IV. Average and spread of these four values are given in the first row of specimen $A$ in Table III. Similarly, the phase angle of specimen $L$ is obtained by the average of $-2.0^{\circ},-1.7^{\circ}$, $-2.0^{\circ}$ and $-1.5^{\circ}$ in the four nulled regions. The relative amplitude of these two specimens are also obtained from Fig. 25 and are shown in the first row of each specimen data of Table III. Data given in the second row of specimens $A$ and $L$ are obtained in the same manner from 
another set of independent measurements similar to Fig. 25. Results in Table III indicate that repeatabilities of these measurements are good. In the third row of each specimen, the averages of two repeatable measurements are averaged again to give the final results which are to be used for calculation of the desired parameters $\left(\mu_{Y}, e_{r}\right)$.

TABLE III

EXAMPLES OF DATA REDUCED FROM THE H-PLANE MEASUREMENTS AT $7.03 \mathrm{GHz}$

\begin{tabular}{|c|c|c|c|}
\hline Specimen & $\begin{array}{c}\text { Average } \\
\text { points }\end{array}$ & \multicolumn{2}{|c|}{$\gamma_{\phi}\left(\theta=120^{\circ}\right)$} \\
\cline { 2 - 4 } & 4 & $-10.45 \pm 0.1$ & $+11.25 \pm 1.25$ \\
\cline { 2 - 4 } & 4 & $-10.5 \pm 0.11$ & $+11.55 \pm 1.2$ \\
\cline { 2 - 4 } & 8 & $-10.48 \pm 0.11$ & $+11.4 \pm 1.25$ \\
\hline \multirow{4}{*}{ A } & 4 & $-4.4 \pm 0.05$ & $-1.8 \pm 0.5$ \\
\cline { 2 - 4 } & 4 & $-4.4 \pm 0.05$ & $-1.7 \pm 0.6$ \\
\cline { 2 - 4 } & 8 & $-4.4 \pm 0.05$ & $-1.75 \pm 0.6$ \\
\hline
\end{tabular}

Data in the first row are obtained from Fig. 25

Data in the second row are obtained from an independently repeated measurements of Fig. 25

Data in the third row are the averages of those in the first two rows.

Our measurement experiences indicate that, in general repeatabilities of better than $U .1 \mathrm{~dB}$ and $1^{\circ}$ can be obtained by careful measure ments. Uncertainties associated with each set of measurements increase 
rapidly when scattered power from a specimen sphere becomes smaller. Finally, we note with care that the uncertainties shown in Table III are strictly those associated with the data read in the four nulled zones. These uncertainties may be higher or lower than those associated theoretically with their null levels. For instance, specimen $A$ in Fig. 25 has null levels of about $-35 \mathrm{~dB}$. If the background noises were assumed random, the theoretical uncertainties would be about $0.15 \mathrm{~dB}$ and $1.5^{\circ}$, respectively, for amplitude and phase. These figures are slightly larger than those in Table III. It should also be noted that random errors could be reduced by taking the average value of a sufficiently large number of measured points.

\section{G. Evalulation Of Parameters}

In the same manner as those data obtained in Table III, we have all together obtained six $y^{\prime}$ s for each specimen at 6.17 and $7.03 \mathrm{GHz}$. These $\gamma^{\prime}$ s are designated as $\left(\gamma_{\theta}\left(90^{\circ}\right), \gamma_{\theta}\left(120^{\circ}\right), \gamma_{\theta}\left(135^{\circ}\right)\right)$ in the Eplane and $\left(\gamma_{\phi}\left(90^{\circ}\right), \gamma_{\phi}\left(120^{\circ}\right), \gamma_{\phi}\left(135^{\circ}\right)\right)$ in the H-plane. Two $\gamma^{\prime}$ 's in E-plane and H-plane, respectively, are used in Eqs. (17) and (18) for preliminary checks on consistency of these measured data. At $6.17 \mathrm{GHz}$, it was found that the combination $\left(\gamma_{\theta}\left(90^{\circ}\right), \gamma_{\phi}\left(90^{\circ}\right)\right)$ and $\left(\gamma_{\theta}\left(135^{\circ}\right), \gamma_{\phi}\left(135^{\circ}\right)\right)$ tends to give best solutions. In other words, the solutions $\left(a_{2}, b_{2}\right)$ produced by this combination are most consistent with the corresponding solutions $\left(a_{1}, b_{1}\right)$. Similarly, the combination $\left(\gamma_{\theta}\left(90^{\circ}\right), y_{\phi}\left(90^{\circ}\right)\right)$ and 
$\left(y_{\theta}\left(120^{\circ}\right), y_{\phi}\left(120^{\circ}\right)\right)$ at $7.03 \mathrm{GHz}$ tended to give best solutions. We summarize in Table IV these two combinations which have been actually used for computation of the parameters $\left(\eta_{r}, \nu_{r}\right)$ or $\left(\mu_{r}, \epsilon_{r}\right)$.

TABLE IV

SUMMARIZED STATEMENTS ON MEASUREMENTS AND COMPUTATIONS

\begin{tabular}{|c|c|c|}
\hline $\begin{array}{c}\text { Frequencies } \\
(G H z)\end{array}$ & $\begin{array}{c}\text { Sizes } \\
\left(\rho_{0}=\pi d / \lambda_{0}\right)\end{array}$ & $\begin{array}{c}\text { Normalized bistatic fields used } \\
\text { in the consistency process }\end{array}$ \\
\hline 6.17 & 0.82112 & $\left(\gamma_{\theta}\left(90^{\circ}\right), \gamma_{\phi}\left(90^{\circ}\right)\right)$ and $\left(\gamma_{\theta}\left(135^{\circ}\right), \gamma_{\phi}\left(135^{\circ}\right)\right)$ \\
\hline 7.03 & 0.93557 & $\left(\gamma_{\theta}\left(90^{\circ}\right), \gamma_{\phi}\left(90^{\circ}\right)\right)$ and $\left(\gamma_{\theta}\left(120^{\circ}\right), \gamma_{\phi}\left(120^{\circ}\right)\right)$ \\
\hline
\end{tabular}

Specimens [8] A, I, K and $L$ have the same diameter $d=1.27 \mathrm{~cm}$. All the specimens are measured at $\theta=90^{\circ}, 120^{\circ}$ and $135^{\circ}$ in both E- and H-planes.

Each of the combinations chosen in Table IV gives rise to a pair of solutions for $\left(a_{2}, b_{2}\right)$ which are in general far from satisfying the consistents criteria described in Chapter IV. This is expected, because our data are far from satisfying the ideal conditions in theory and measurement. Every $y$ in each of the combinations carries its own uncertainties depending on measurement conditions. Uncertainties in some $\gamma^{\prime} s$ are higher than the others.

In order to improve our measured data, we make some preliminary adjustments first to the most uncertain $\gamma$ in each combination. For example, if the most uncertain $y$ has $\pm 0.3 \mathrm{~dB}$ uncertainty we let 
this $y$ be changed by $\pm 0.1 \mathrm{~dB}$ and solve for the undetermined coefficients by Eq. (17). If the change of $-0.1 \mathrm{~dB}$ improves the consistency after use of Eq. (18), then an additional change of $-0.1 \mathrm{~dB}$ is introduced. Should this additional change of $-0.1 \mathrm{~dB}$ to the $\gamma$ improve the consistency further, then a total of but no more than $-0.3 \mathrm{~dB}$ may be used to adjust this $y$. Should the additional change of $-0.1 \mathrm{~dB}$ fail to improve the consistency further, then only the original change of $-0.1 \mathrm{~dB}$ is allowed to this $\gamma$. The next step is to adjust the phase angle of this $\gamma$ in steps of $1^{\bullet}$ to improve the consistency. Procedures in phase adjustment are similar to those described above in amplitude adjustment. After the most uncertain $\gamma$ is adjusted, the consistency is in general improved considerably. Should the improved consistency fall within the consistency criteria imposed in Chapter IV, it is now permissible to use the consistency process to this particular combination of $\gamma^{\prime} s$. Otherwise, it is necessary to adjust the second most uncertain $y$ for improvement. The process may be continued until every $y$ is adjusted. It is important, however, to note that no total adjustment to $a \gamma$ is allowed to exceed the amount of uncertainties associated with this $y$ from measurements. Evidently, this is because the entire adjustments would be rendered meaningless in the sense that accuracies of the subsequent solutions would have been improved beyond measurement accuracies. Also it is important, on the other hand, to note that proper adjustments to the $\gamma^{\prime}$ s do not necessarily guarantee 
a set of solutions which would satisfy the consistency criteria imposed in Chapter IV. Our data obtained at $7.03 \mathrm{GHz}$ for the $y$ 's of specimen I will serve to indicate that it is possible to obtain no consistent result even after the measurement uncertainties are adjusted.

Other sequences of preliminary adjustments, different from the one just mentioned above, can also be employed. They would naturally lead to different results. Although these different sequences of adjustments have not been pursued at this time, one may refer to three different sets of data in Table I and examine the different results in Table II.

The effects of preliminary adjustments mentioned above are that uncertainties in each $y$ are reduced individually. When the preliminary adjustments finally give rise to a set of solutions whose $\left(a_{2}, b_{2}\right)$ are consistent with $\left(a_{1}, b_{1}\right)$ to within the consistency criteria imposed in Chapter IV, the uncertainties of the $\gamma$ 's can then be reduced collectively by applying the consistency process.

Those $y$ 's resulted from preliminary adjustments are first considered as if they were the true values that could be expected under ideal conditions. These $\gamma^{\prime} s$ are then listed in the same manner as that of row la data in Table I. In order to apply the consistency process, the $\gamma^{\prime}$ s are then shifted $-0.025 d B$ and $-0.25^{\circ}$, respectively, in the same manner as those shifted data in row $3 a$ of Table $I$. 
By following the examples given in Chapter IV, we show in Figs. 28 to 31 the distributions of $\eta_{r}$ for the four specimens measured at $6.17 \mathrm{GHz}$. One notes that these distributions are all confined in a $1 \%$-circle centered at the estimated values of $\eta_{\mathbf{r}}$. The corresponding distributions of $v_{x} p_{0}$ for specimens $A$ and $I$ are also confined in a $1 \%$ circle centered at the estimated values of $v_{x} P_{0}$. These distributions are not to be shown because they are too crowded in the refractive index chart. In Fig. 32, however, we show the distributions of $\mathrm{X}_{1}$ for specimens $K$ and $L$ at $6.17 \mathrm{GHz}$. Transformations of the $X_{1}$ distributions to $v_{r} \rho_{0}-p$ lane are shown in Figs. 33 and 34 where one

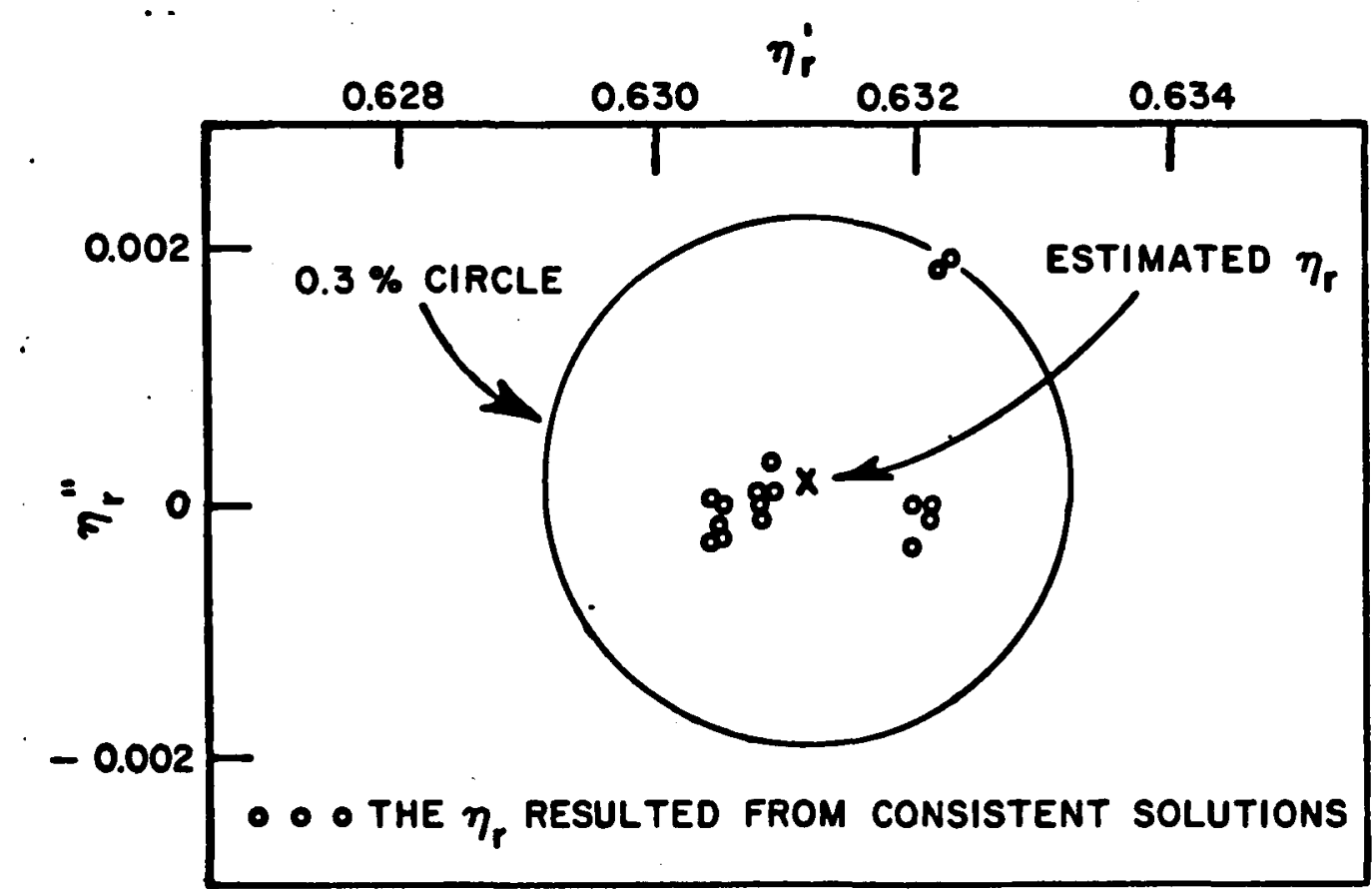

Fig. 28--Distribution of $\eta_{r}$ from the consistency process applied to specimen $\mathrm{A}$ measured at $6.17 \mathrm{GHz}$. 


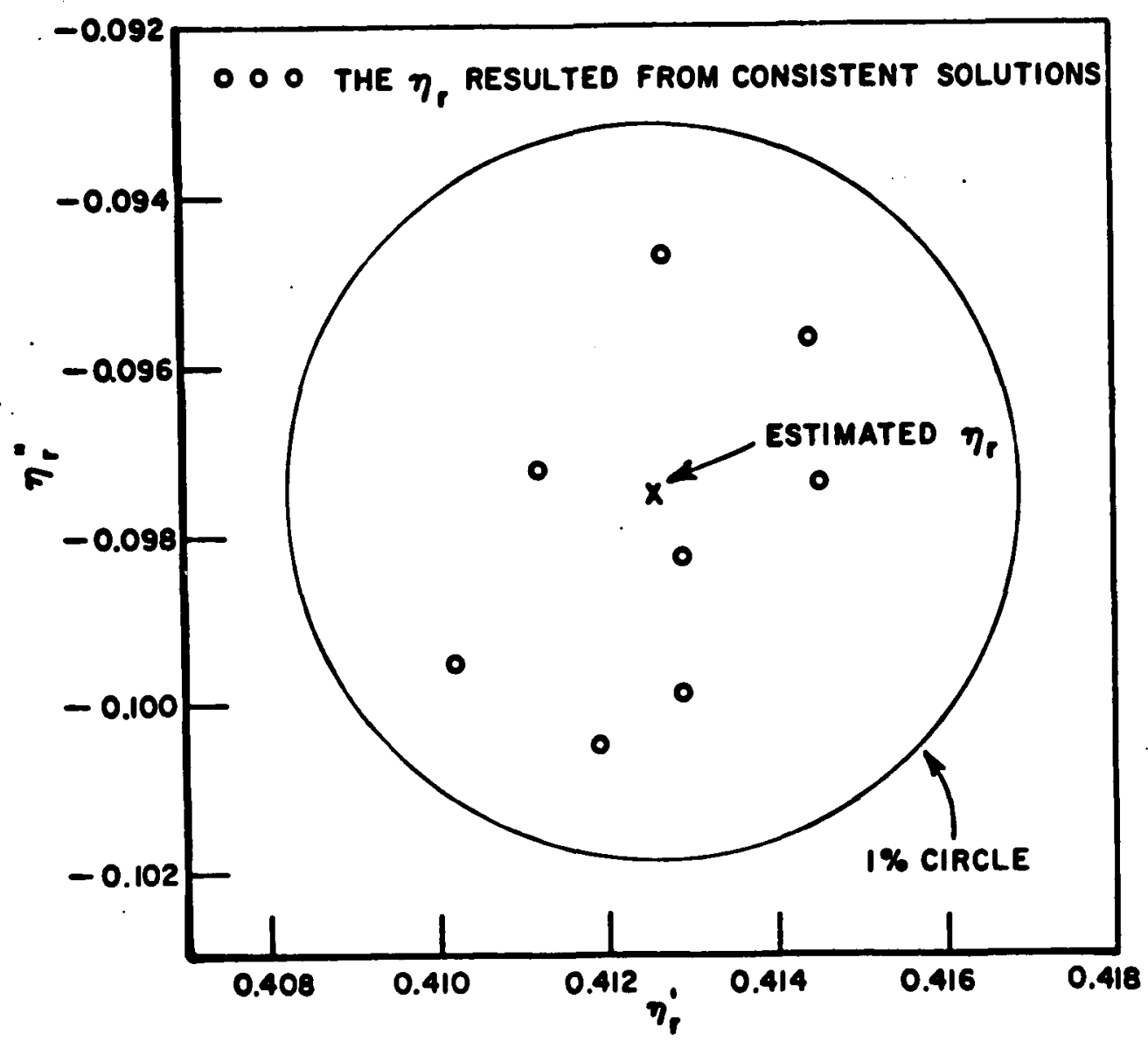

Fig. 29--Distribution of $\eta_{x}$ from the consistency process. applied to specimen I measured at $6.17 \mathrm{GHz}$.

see larger percentage uncertainties for the distributions of $v_{r} \rho_{0}$. For instance, consider specimen $\mathrm{K}$. While $\mathrm{X}_{1}$ in Fig. 32 indicates an uncertainty of $1 \%$ of the estimated value, the transformed $v_{\mathbf{r}} \rho_{0}$ in Fig. 33 results in an uncertainty of about $3 \%$ of the estimated value. The estimated values of $\left(\eta_{Y}, v_{Y}\right)$ obtained at $6.17 \mathrm{GHz}$ are summarized in Table V. Evidently, the values of $v_{r}$ are obtained by dividing those estimated $v_{Y} \rho_{0}$ by $P_{0}=0.82112$ for $6.17 \mathrm{GHz}$. The results obtained for $7.03 \mathrm{GHz}$ are also given in the same Table. No result is 


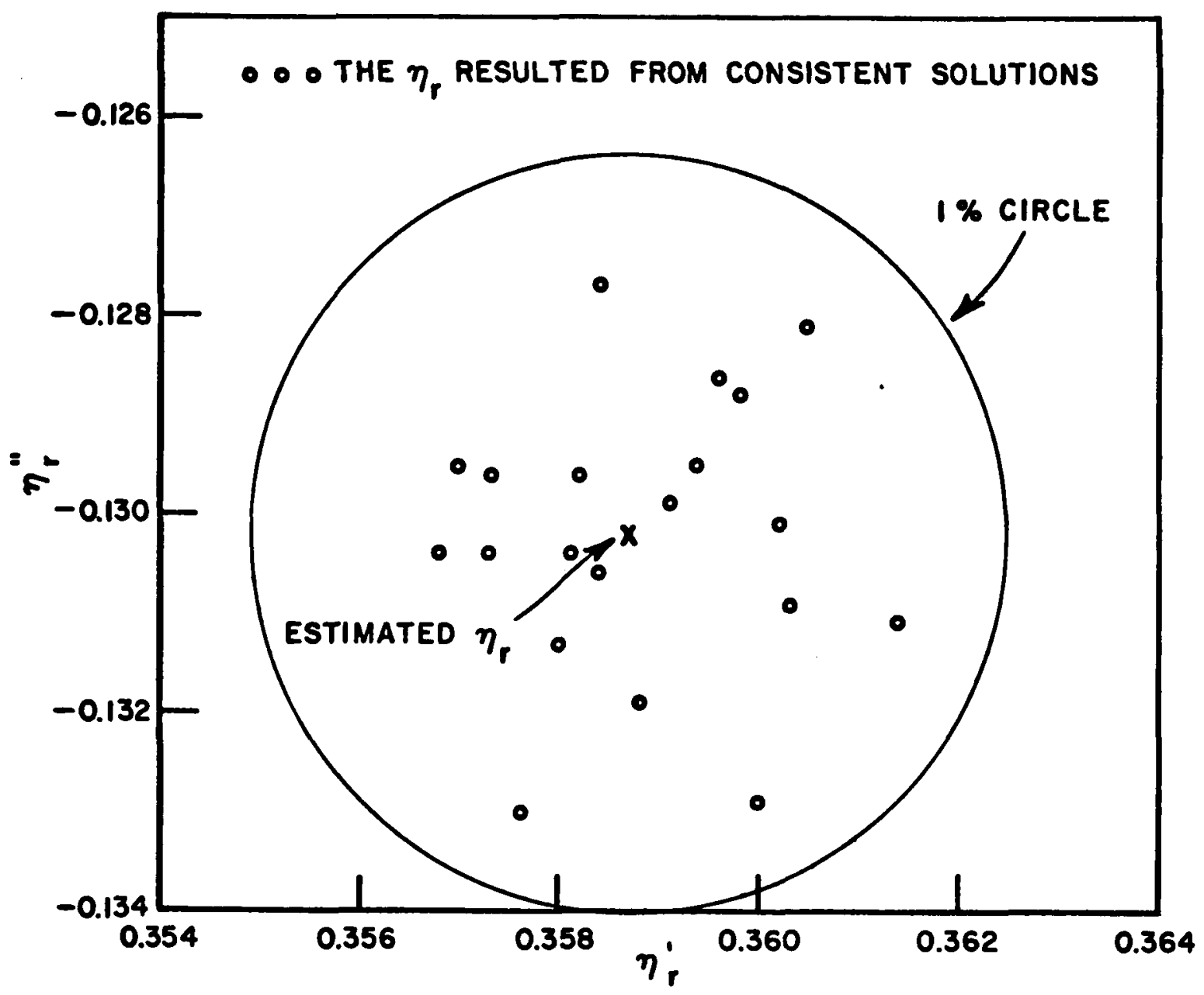

- Fig. 30--Distribution of $\eta_{r}$ from the consistency process applied to the specimen $\mathrm{K}$ measured at $6.17 \mathrm{GHz}$.

given in Table $V$ for specimen $I$ at $7.03 \mathrm{GHz}$, because its measured data in the combination chosen in Table IV failed to give consistent solutions through the preliminary adjustments. We have chosen the best of the nonconsistent solutions to give $\left(\mu_{r},{ }{ }_{r}\right)$ of specimen I at 7.03 GHz as shown in Table VI. In this Table, we list the values of $\left(\mu_{r}, e_{r}\right)$ for the four specimens at three frequencies. The values given for $8.6 \mathrm{GHz}$ or $9.27 \mathrm{GHz}$ are taken from the method[10] of single-double thickness transmission in waveguide. 


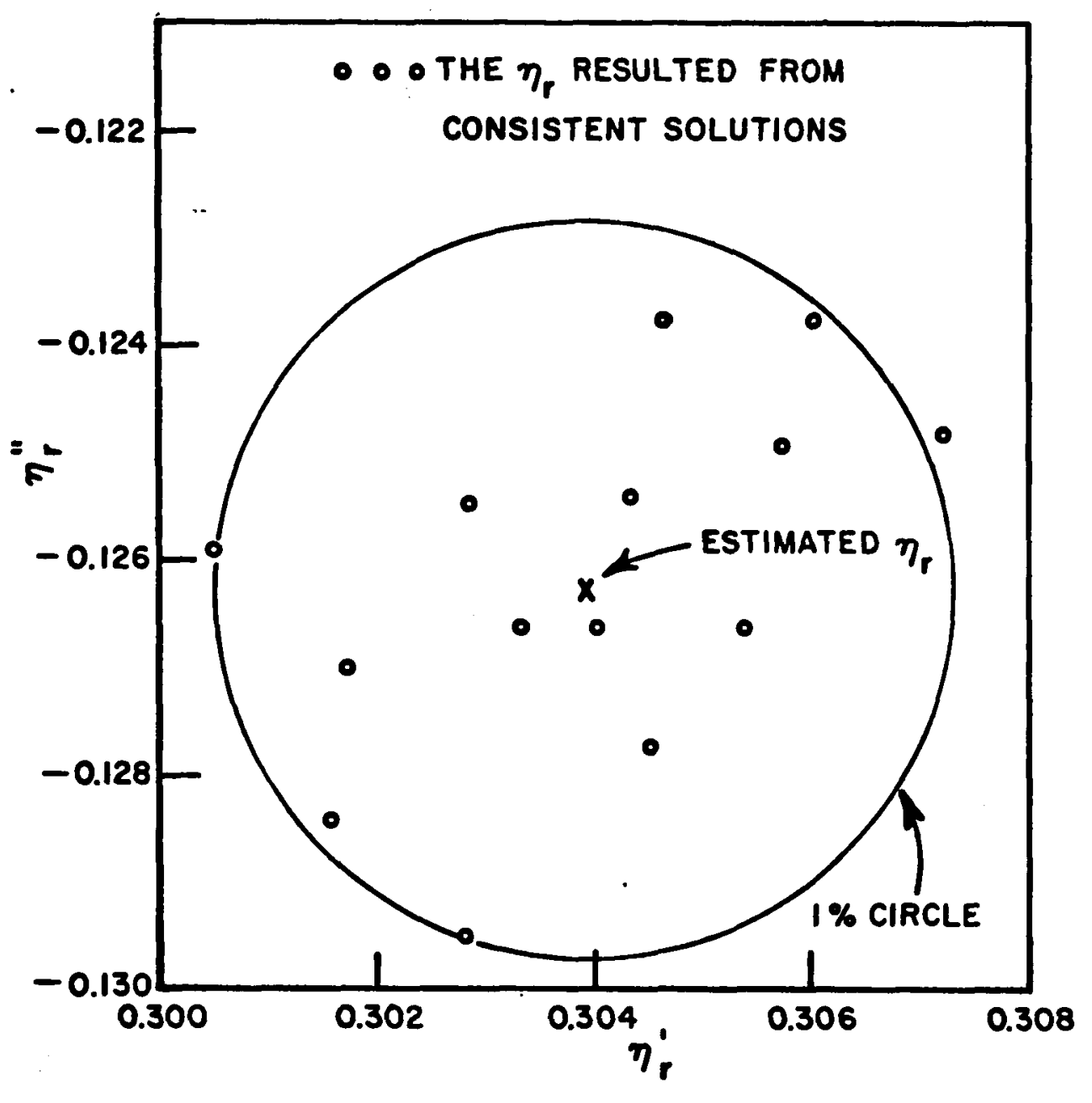

Fig. 31--Distribution of $\eta_{r}$ from the consistency process applied to the specimen $\mathrm{L}$ measured at $6.17 \mathrm{GHz}$. 


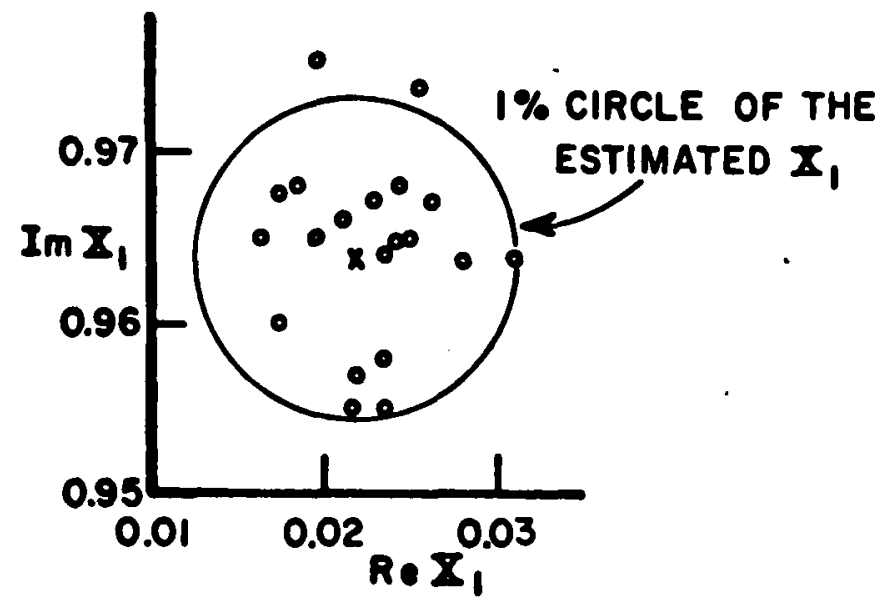

(a) SPECIMEN $K$ : ESTIMATED $X_{1}=0.022+j 0.964$

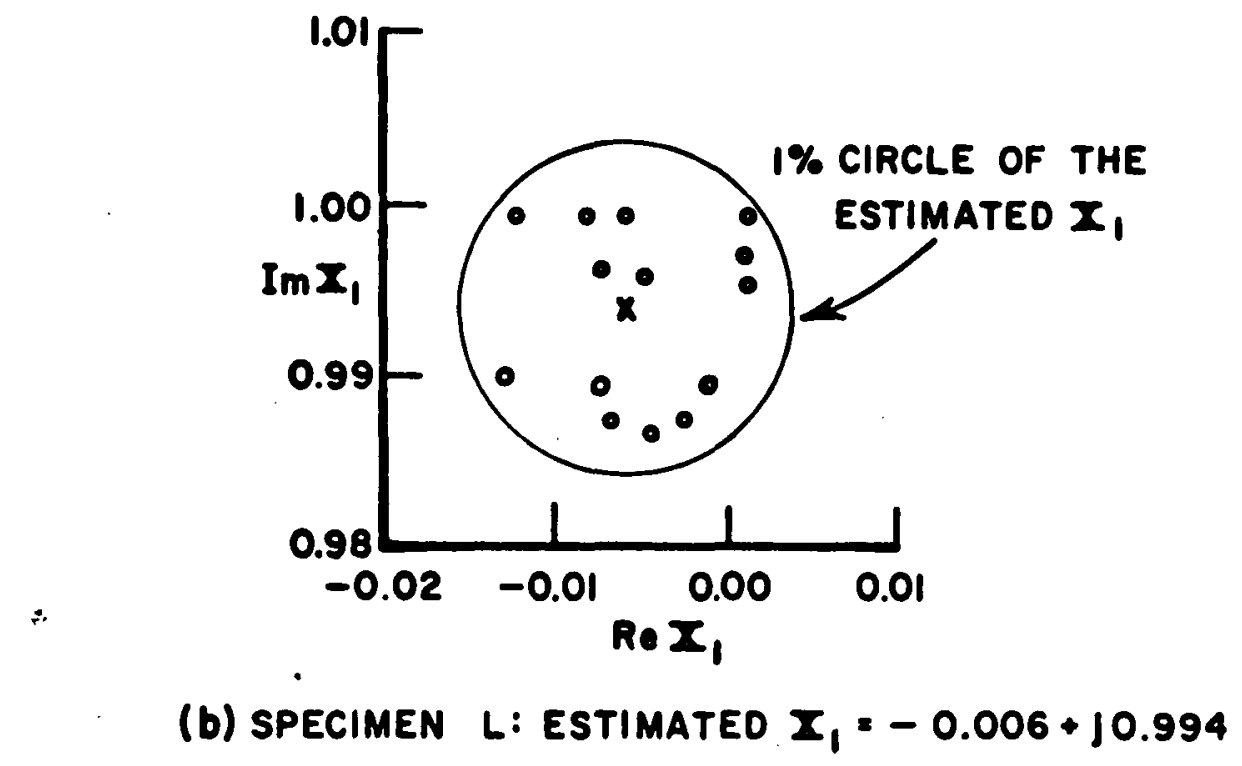

Fig. 32--Distributions of $X_{1}$ from the consistency process applied to specimens $K$ and $L$ measured at $6.17 \mathrm{GHz}$. 


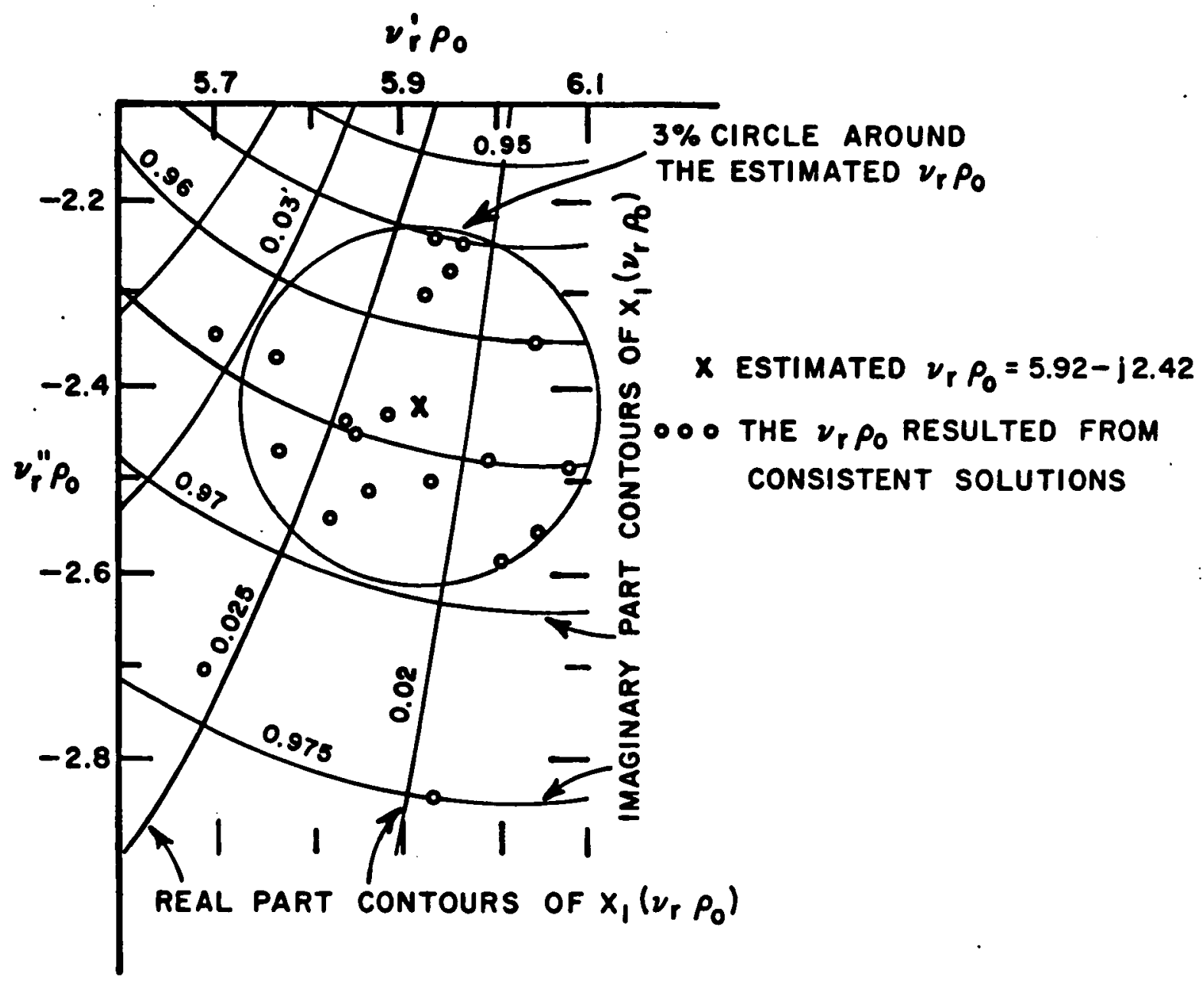

Fig. 33 --Distribution of $\nu_{r} p_{0}$ from the consistency process applied to specimen $\mathrm{K}$ measured at $6.17 \mathrm{GHz}$.

H. Assessment Of The Parameters

Any attempt to quantitatively describe the inaccuracy of the final results in Table VI would involve high risk of being in error, because we did not make a sufficient number of independent measurements to invoke statistical concepts. In other words, at the present stage of investigation we are not able to estimate with confidence the percentage inaccuracies of our final values of $\left(\mu_{r}, \epsilon_{r}\right)$. However, it is 


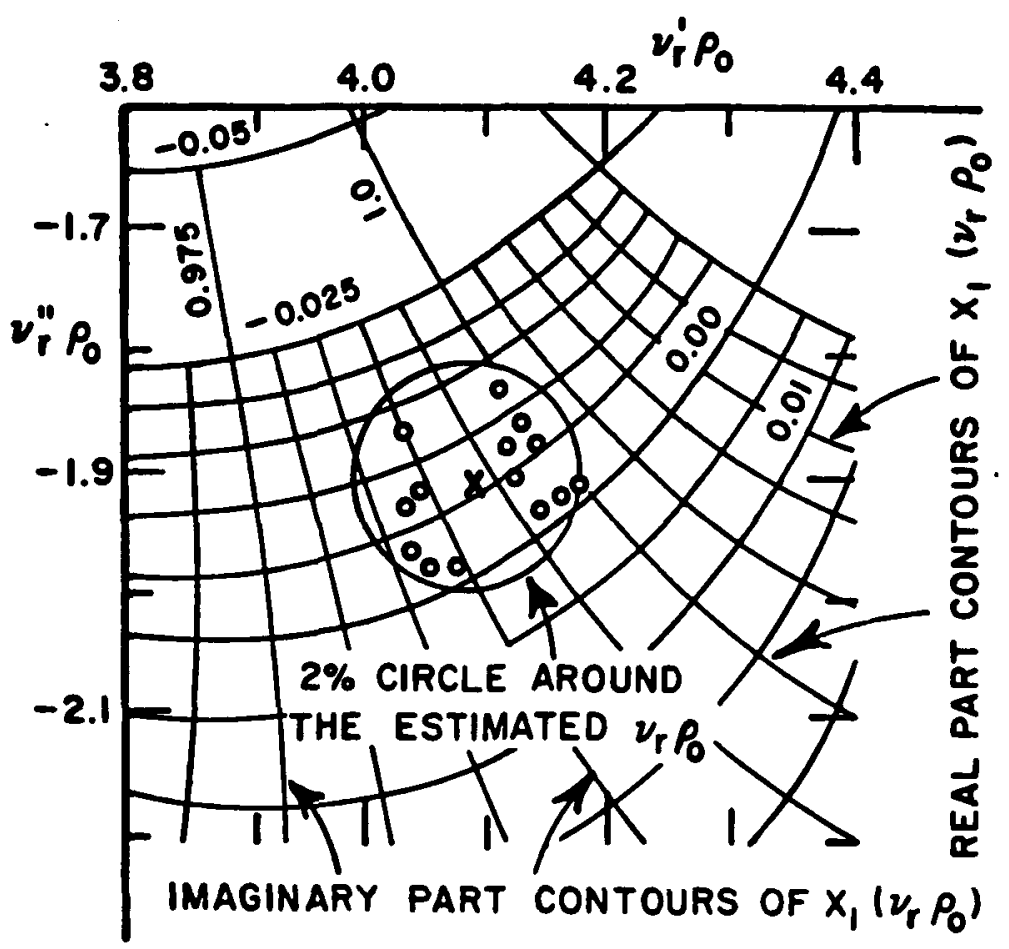

\section{$X \times$ ESTIMATED $\nu_{1} p_{0}=4.09-j 1.91$ \\ - 0 THE $\nu_{r} P_{0}$ RESULTED FROM CONSISTENT SOLUTIONS}

- Fig. 34--Distribution of $v_{r} P_{0}$ from the consistency process applied to specimen $\mathrm{L}$ measured at $6.17 \mathrm{GHz}$.

known that specimen $A$ (cross-linked polystyrena) [8] has a fairly frequency-independent $\epsilon_{r}$. The values of $\left(\mu_{r}, \epsilon_{r}\right)$ obtained for specimen $A$ in Table VI indicate that our scattering method is capable of measuring this type of materials accurate enough to compare with a variety of methods reported in Reference 8 .

As far lossy materials, specimens $I, K$ and $L$, a convenient and reliable method for determining inaccuracies in $\left(\mu_{r}, \epsilon_{r}\right)$ is to compare the experimentally measured data with theoretical bistatic patterns which are computed by use of the parameters in Table VI. We use the 
TABLE V

ESTIMATED VALUES OF $\left(\eta_{Y}, v_{r}\right)$ OBTAINED BY USE OF THE COMBINATIONS IN TABLE IV IN THE CONSISTENCY PROCESS

\begin{tabular}{|c|c|c|c|c|}
\hline Specimen & Freq. & Estimated $\eta_{\mathbf{r}}$ & Estimated $\mathbf{X}_{1}$ & Estimated $v_{\mathbf{r}} \rho_{0}$ \\
\hline \multirow{2}{*}{$\mathbf{A}$} & 6.17 & $0.6321+j 0.0002$ & $1.254+\mathrm{j} 0.009$ & $\rho_{0}(1.59 .3-\mathrm{j} 0.0097)$ \\
\hline & 7.03 & $0.6275+j 0.001$ & $1.025+\mathrm{j} 0.006$ & $\rho_{0}(1.589-\mathrm{j} 0.005)$ \\
\hline \multirow{2}{*}{ I } & 6.17 & $0.4126-\mathrm{j} 0.0975$ & $-0.324+j 0.905$ & $\rho_{0}(4.518-j 1.12)$ \\
\hline & 7.03 & \multicolumn{2}{|c|}{ No consistency from measured data } & \\
\hline \multirow{2}{*}{$\mathbf{K}$} & 6.17 & $0.3578-\mathrm{j} 0.1302$ & $0.0225+j 0.963$ & $p_{0}(7.21-j 2.95)$ \\
\hline & 7.03 & $0.3502-\mathrm{j} 0.1129$ & $0.004+j 0.959$ & $\rho_{0}(6.35-j 2.54)$ \\
\hline \multirow{2}{*}{$\mathbf{L}$} & 6.17 & $0.3039-\mathrm{j} 0.1262$ & $0.0055+j 0.993$ & Pol(4.98-j2.31) \\
\hline & 7.03 & $0.2860-\mathrm{j} 0.1018$ & $0.025+j 0.966$ & Pol (5.34-j 2.35) \\
\hline
\end{tabular}

$$
\begin{aligned}
\rho_{0}=\pi d / \lambda_{0} & =0.82112 \text { at } 6.17 \mathrm{GHz} \\
& =0.93557 \text { at } 7.03 \mathrm{GHz}
\end{aligned}
$$


TABLE VI

THE ESTMMATED VALUES OF ( $\left.\mu_{r}, e_{r}\right)$ OBTAINED FROM THE ESTMMATED $\left(\eta_{r}, v_{r}\right)$ IN TABLE $V$

\begin{tabular}{|c|c|c|c|c|c|c|}
\hline \multirow{2}{*}{ Specimen } & \multirow{2}{*}{$\begin{array}{l}\text { Freq. } \\
\text { (GHz) }\end{array}$} & \multicolumn{2}{|c|}{$\mu_{r}=\eta_{r} v_{r}$} & \multicolumn{2}{|c|}{$\epsilon_{r}=\nu_{r} / \eta_{r}$} & \multirow{2}{*}{$\begin{array}{l}\text { Qualitative } \\
\text { Assessment }\end{array}$} \\
\hline & & $\left|\mu_{r}\right|$ & $-\tan \delta_{\mu}$ & $\left|e_{\mathbf{r}}\right|$ & $-\tan \delta_{c}$ & \\
\hline \multirow{3}{*}{$\mathbf{A}$} & 6.17 & 1.005 & 0.0058 & 2.524 & 0.0064 & good \\
\hline & 7.03 & 0.998 & 0.0028 & 2.532 & 0.0064 & good \\
\hline & $9.27 *$ & \multicolumn{2}{|c|}{$\mu_{r}$ assumed unity } & 2.52 & 0.0 & \\
\hline \multirow{3}{*}{ I } & 6.17 & 1.974 & 0.514 & 10.98 & 0.011 & good \\
\hline & $7.03^{* *}$ & 1.825 & 0.45 & 11.23 & 0.102 & uncertain \\
\hline & $8.6^{*}$ & 1.49 & 0.555 & 11.06 & 0.025 & \\
\hline \multirow{3}{*}{$\mathbf{K}$} & 6.17 & 2.972 & 0.907 & 20.41 & 0.039 & good \\
\hline & 7.03 & 2.67 & 0.81 & 19.8 & 0.036 & fair \\
\hline & $8.6 *$ & 2.0 & 0.972 & 20.67 & 0.083 & \\
\hline \multirow{3}{*}{$\mathbf{L}$} & 6.17 & 1.81 & 1.09 & 16.7 & 0.042 & good \\
\hline & 7.03 & 1.77 & 0.945 & 19.3 & 0.073 & uncertain \\
\hline & $8.6^{*}$ & 1.57 & 1.17 & 27.82 & 0.002 & \\
\hline
\end{tabular}

* Data at these frequencies are listed from Reference 8 for convenient comparison

** The measured data at this frequency yield no consistent solution 
parameters listed for $6.17 \mathrm{GHz}$ in Table VI, and compute the theoretical amplitude and phase patterns for all four specimens as shown in Figs. 35 to 42 . These patterns are obtained by use of Eq. (12) first, and then they are normalized by those obtained by use of Eq. (13). Together with these patterns are six measured amplitudes and six measured phase angles. Recall that only four amplitudes and four phase angles, as the combination chosen in Table IV, are used in deduction of the parameters in Table VI. The unused data in amplitude and phase are seen in these figures to deviate more from computed patterns than those that have been used. This is expected, because the combination shown in Table IV was chosen on the basis that it tends to give more consistent solutions. In general, however, all the measured data at $6.17 \mathrm{GHz}$ agree with the computed patterns to within their measurement uncertainties. Therefore, it is reasonable to conclude that these parameters obtained at $6.17 \mathrm{GHz}$ have good accuracy. Hence, a qualitative assessment of good is given to these parameters in Table VI.

Confidence in these results could have been increased if the following possibilities had been considered. First, the constitutive parameters could have been adjusted in small increments about their final values and the curves similar to Figs. 35 to 42 could again have been plotted and compared to the experimental results. Increased discrepancies would then indicate which values of the constitutive 


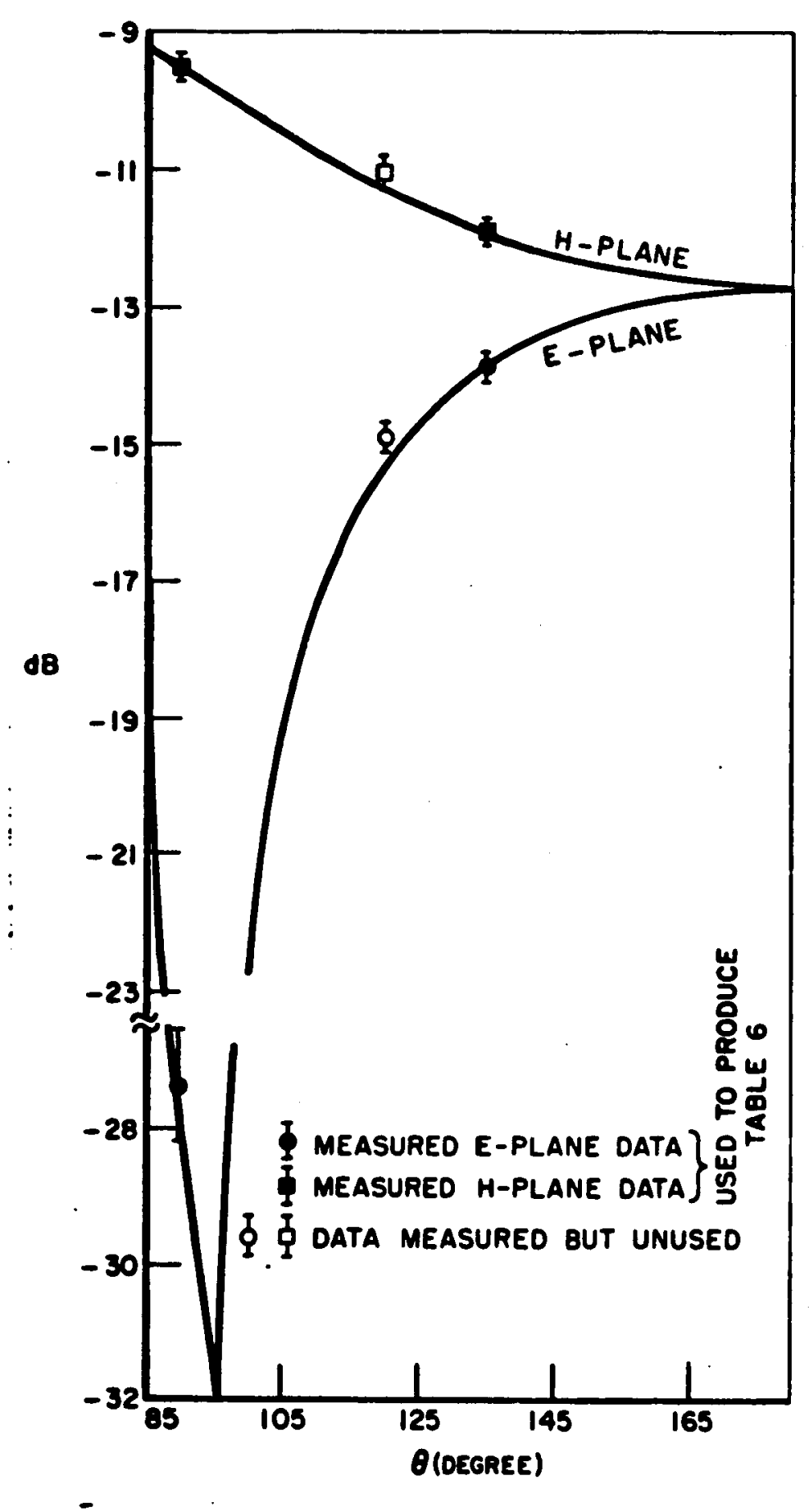

Fig. 35--Normalized bistatic amplitude patterns of specimen $A$ at $6.17 \mathrm{GHz}$ (computed by use of the data in Table VI). 


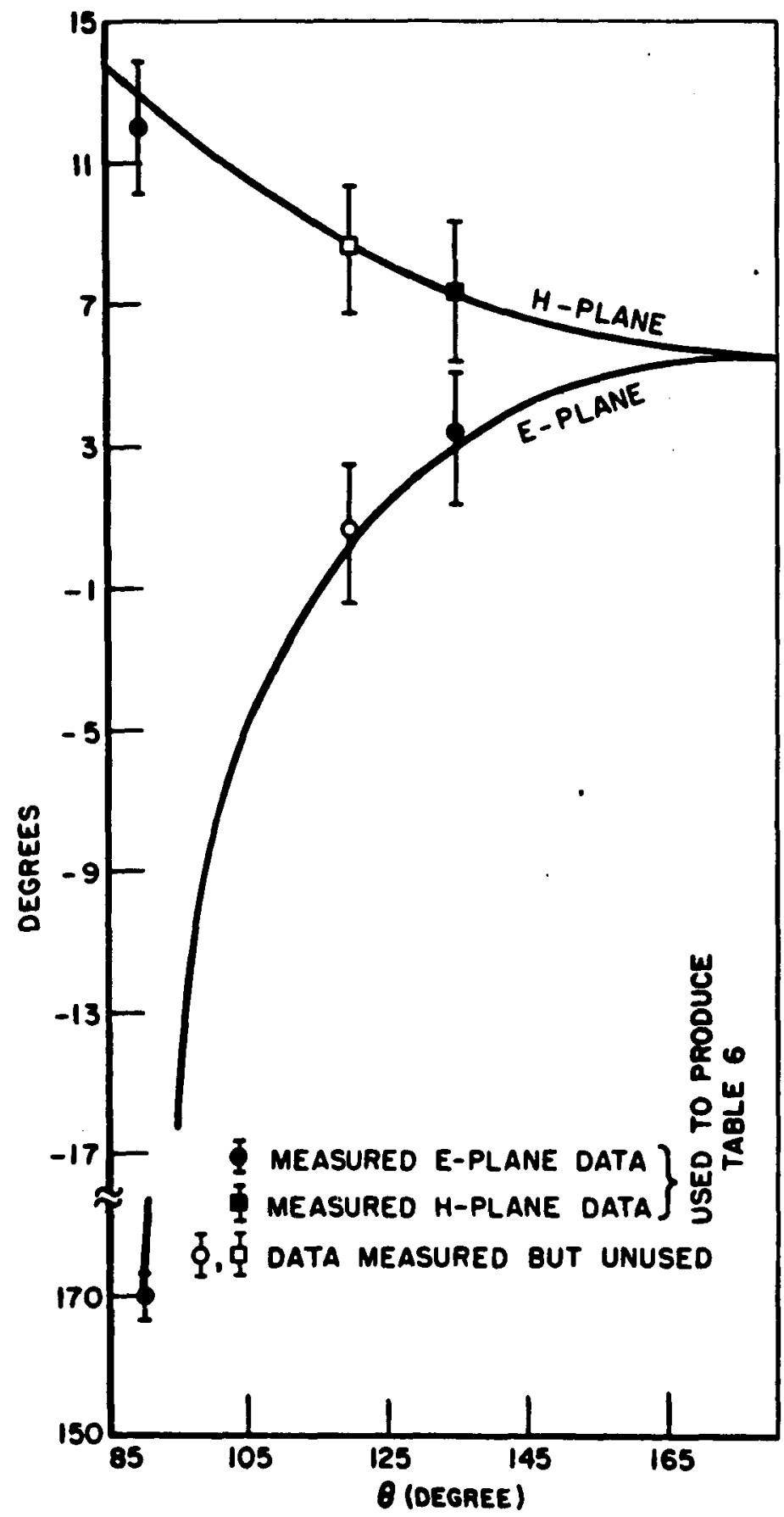

Fig. 36--Normalized bistatic phase patterns of specimen A at $6.17 \mathrm{GHz}$ (computed by use of the data in Table VI). 


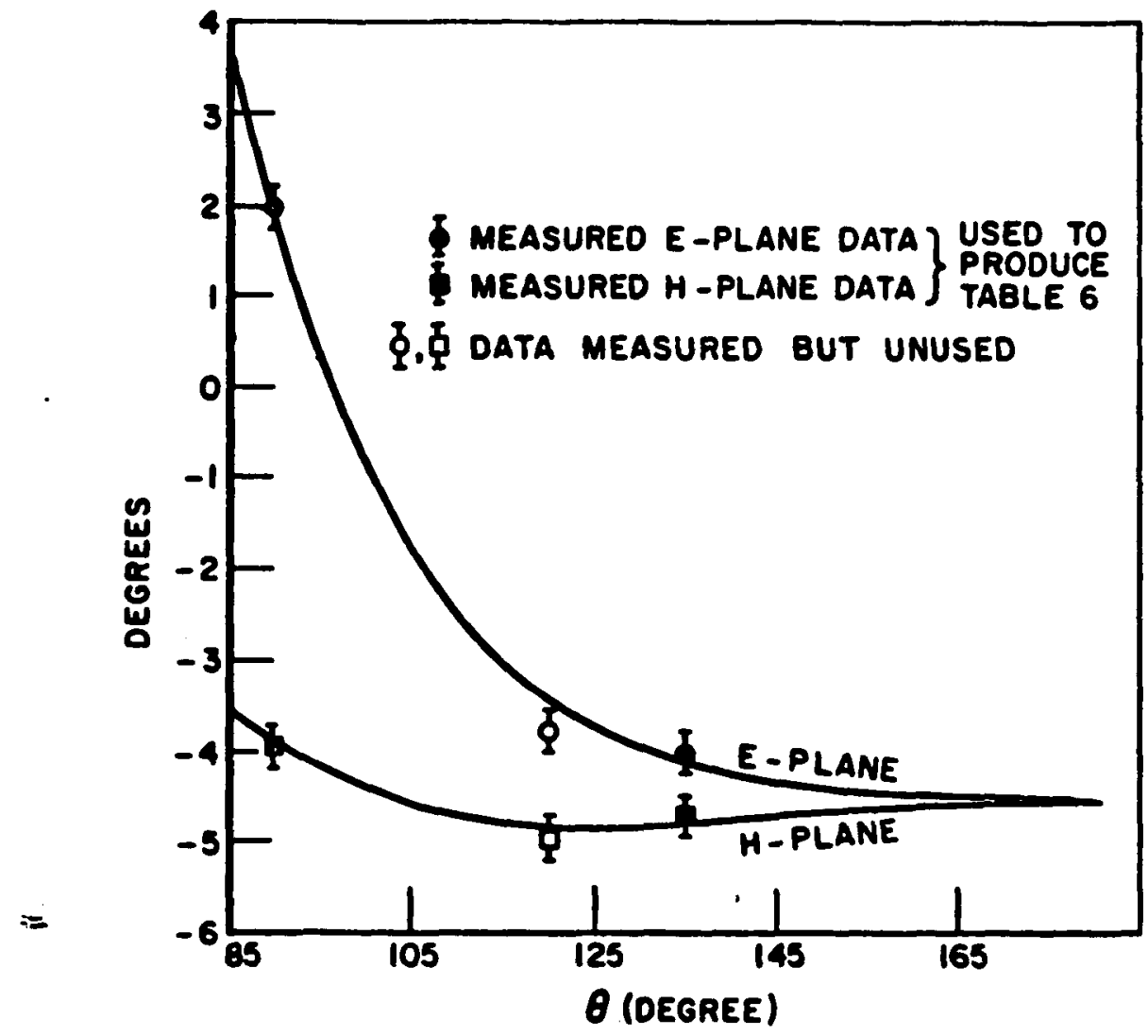

-Fig. 37--Normalized bistatic amplitude patterns of specimen I at $6.17 \mathrm{GHz}$ (computed by use of the data in Table VI).

parameters are most accurate. Second these patterns could be computed with measured data for spheres of different radii provided a sufficient amount of the sample material is available. Larger spheres would yield patterns with greater variation and said patterns would in the lower loss cases depend more cirtically on the constitutive parameters. These processes would be expensive and have not been pursued at this time. However there is considerable merit to such ideas if it is desirable to obtain ever increasing accuracy in the end results. 


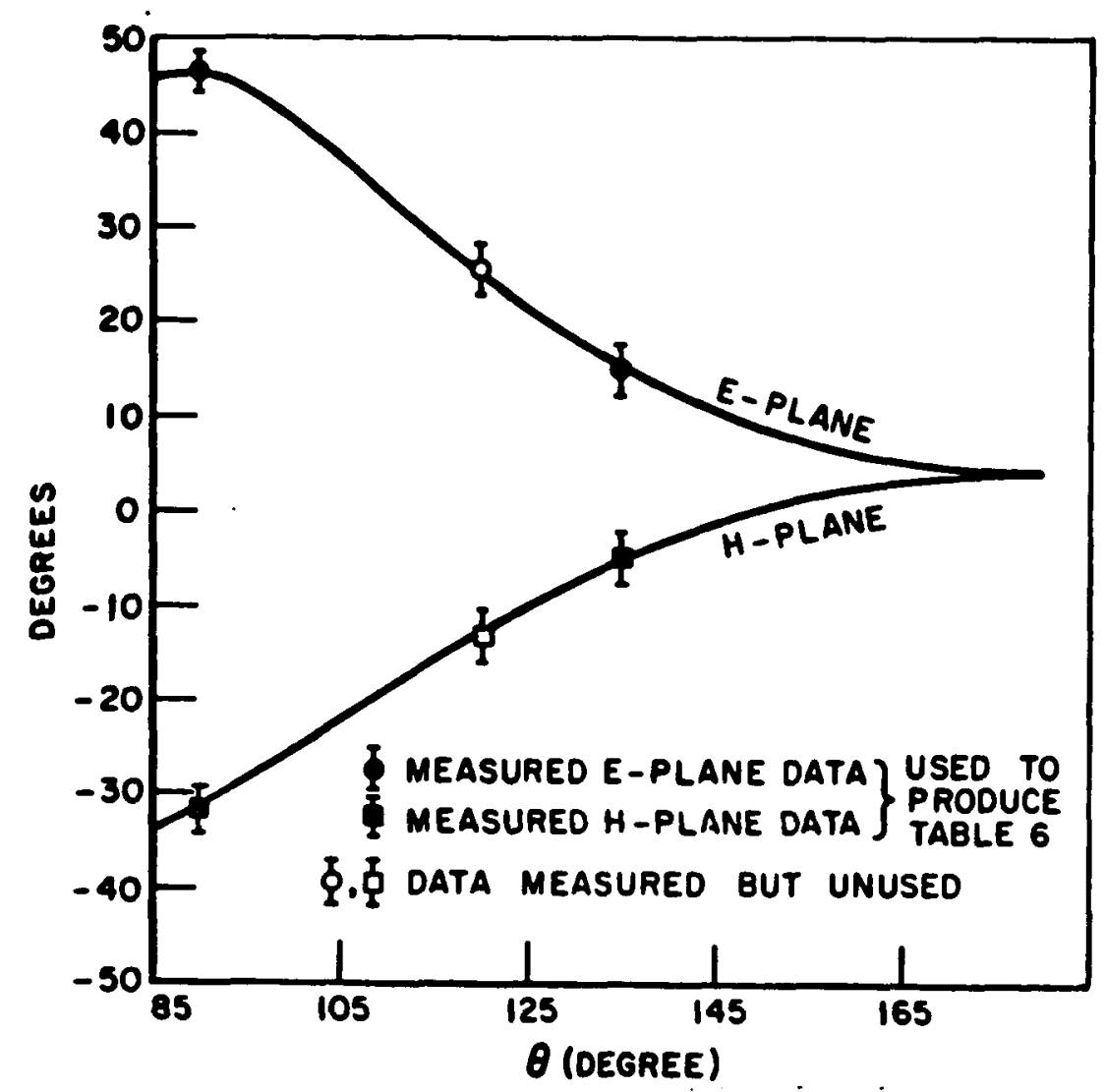

- Fig. 38--Normalized bistatic phase patterns of specimen I at $6.17 \mathrm{GHz}$ (computed by use of the data in Table VI).

Patterns in Figs. 43 and 44 are computed by use of the nonconsistent parameters obtained for specimen I at $7.03 \mathrm{GHz}$. These patterns are shown to illustrate the result of such an inconsistent estimate. The phase patterns in Fig. 44 still agree with the measured data to within their measurement uncertainties. In Fig. 43, the measured amplitude data are not in good agreement with the computed patterns. Because of inconsistency in parameter deduction and disagreement in Fig. 43, we give an assessment of uncertain for those parameters obtained in Table VI. 


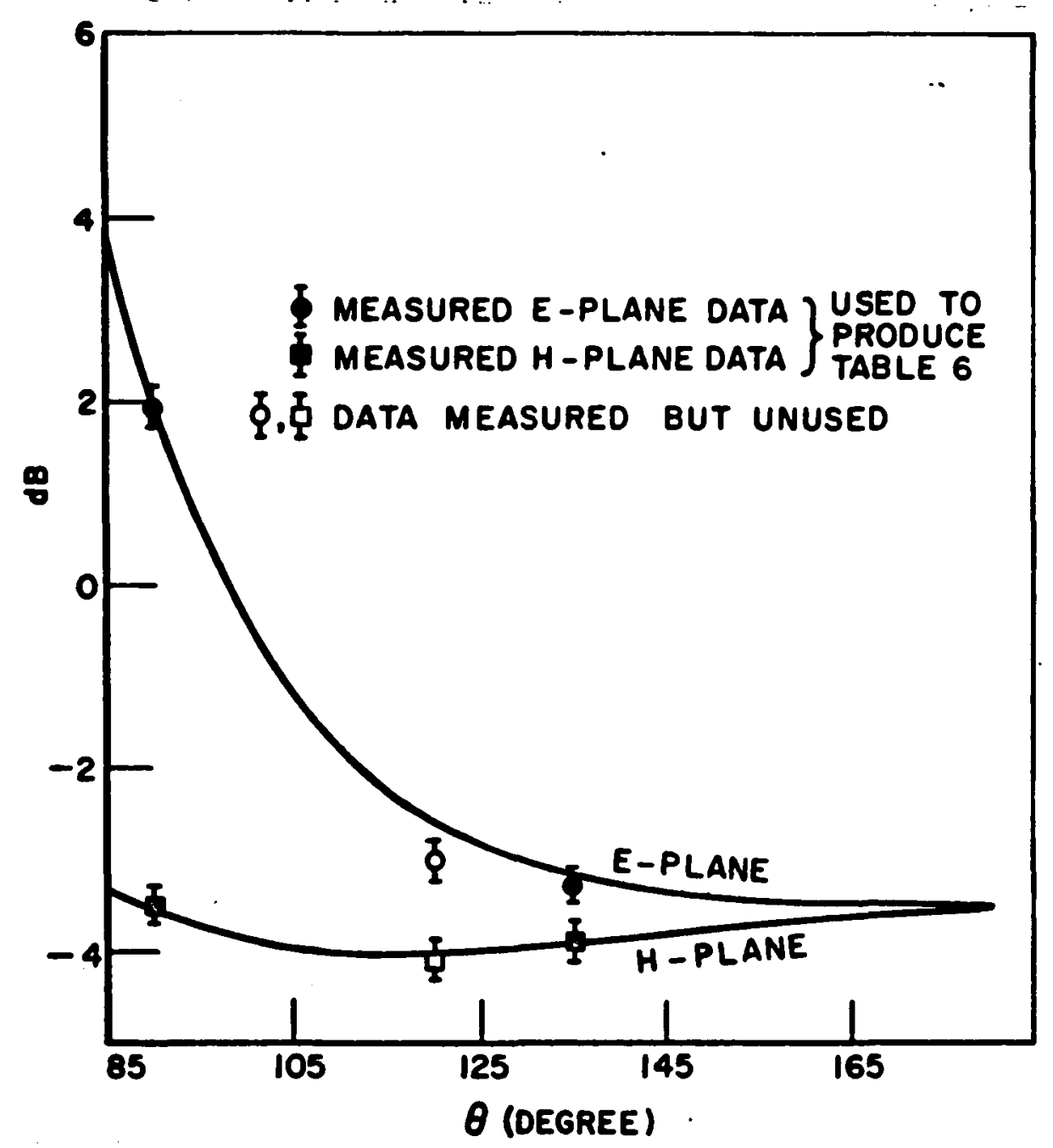

Fig. 39--Normalized bistatic amplitude patterns of specimen $\mathrm{K}$ at $6.17 \mathrm{GHz}$ (computed by use of the data in Table VI).

The parameters of specimen $\mathrm{L}$ at $7.03 \mathrm{GHz}$ are also assessed uncertain, because their theoretical patterns deviate from their measured data in the same extent as those shown in Fig. 43. There is, however, one basic difference in that that consistency criteria were satisfied by its measured data during parameter deduction. Therefore, we have here an example that the consistency process does 


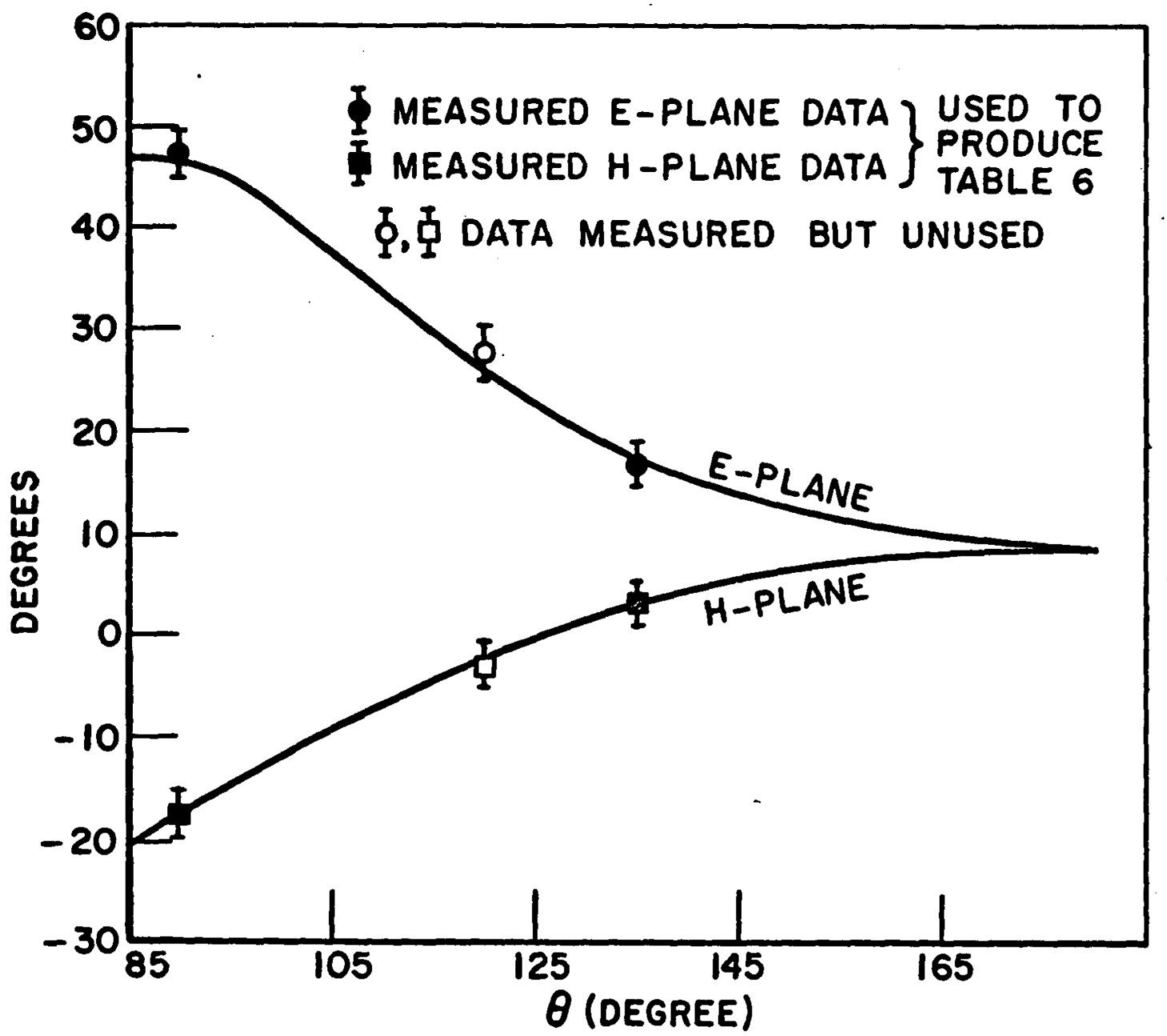

Fig. 40--Normalized bistatic phase patterns of specimen $\mathrm{K}$ at $6.17 \mathrm{GHz}$ (computed by use of the data in Table VI).

not necessarily guarantee accurate results. The reason is that the value of $X_{1}(0.025+j 0.966)$ shown in Table $V$ is very close to the first saddle-point region shown in Fig. 7. Consequently, a set of fairly certain values of $X_{1}$ in the neighborhood of $(0.025+j 0.966)$ is transformed into a set of highly uncertain values of solution, as exemplified in the transormation from Fig. 7 to Fig. 8. 


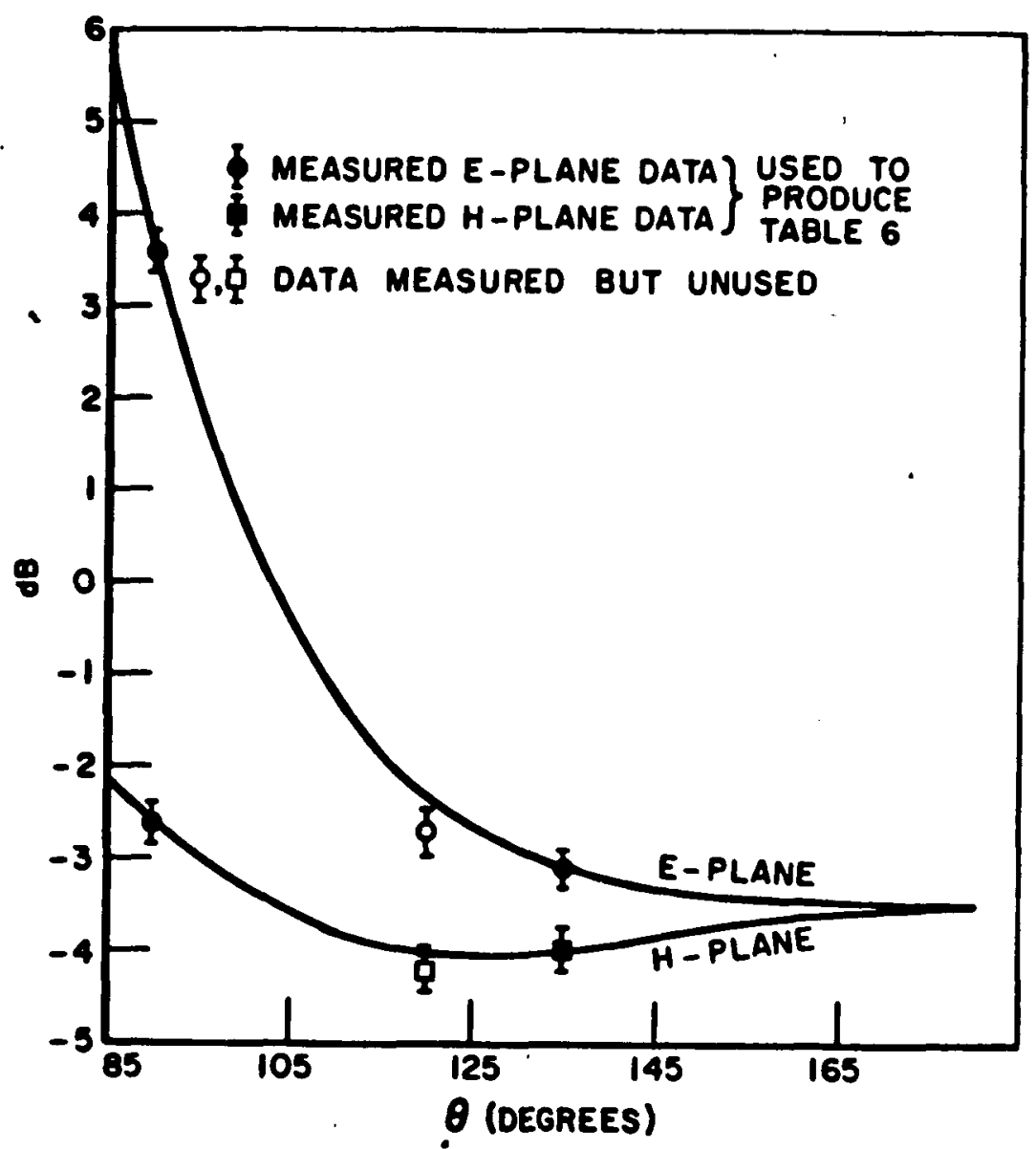

Fig. 41--Normalized bistatic amplitude patterns of specimen $L$ at $6.17 \mathrm{GHz}$ (computed by uge of the data in Table VI).

The parameters obtained for specimen $\mathrm{K}$ at $7.03 \mathrm{GHz}$ are considered fairly accurate. Theoretical patterns obtained by use of these parameters agree with the measured data.much better than those shown in Fig. 43. Nevertheless, their agreements do not appear to be as good as those in Figs. 35 to 42 .

The above comparisons between theoretical patterns and measured data have helped to obtain semi-quantitative assessments on the accuracy of our final results. In order to improve the results of 


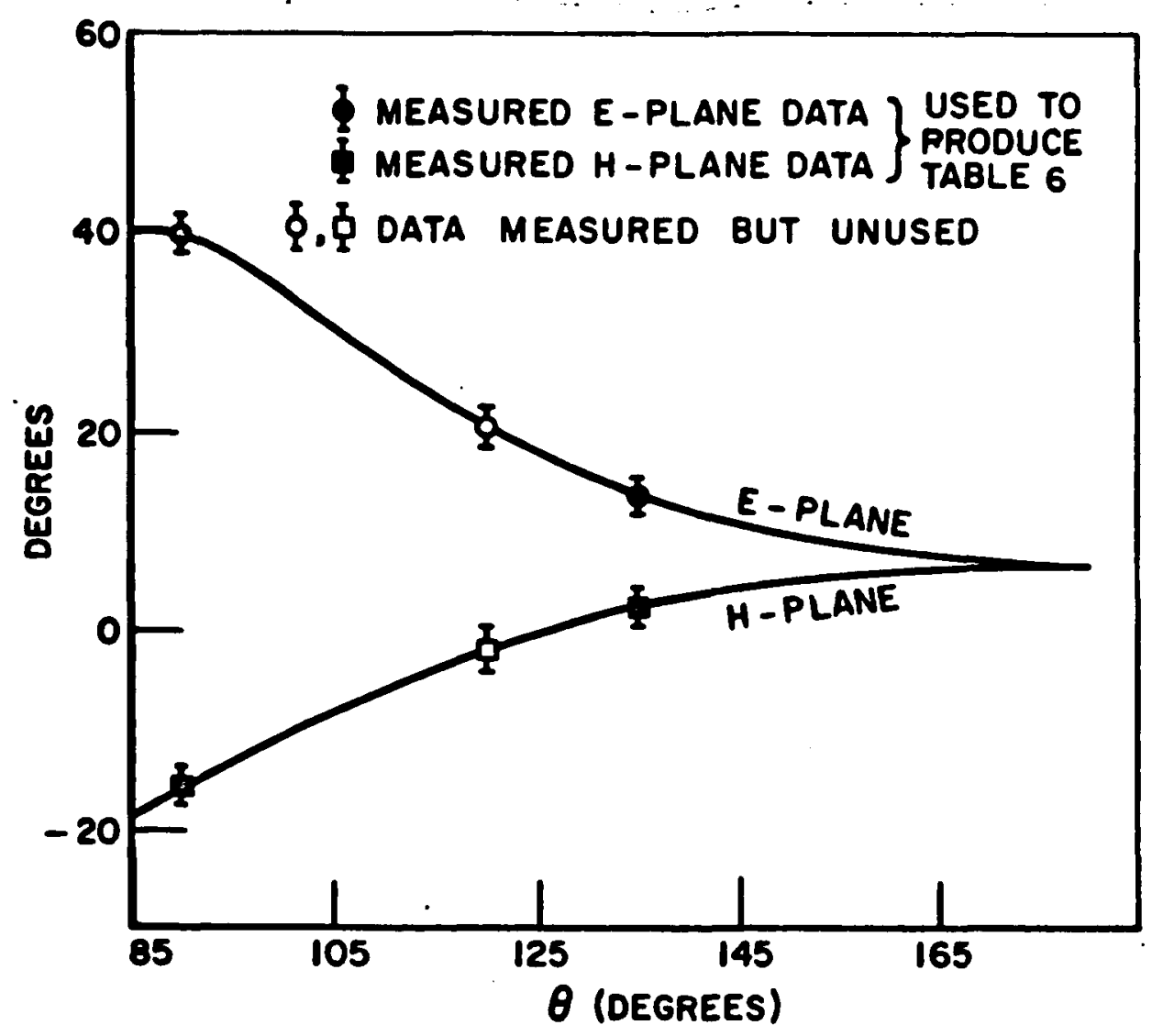

Fig. 42--Normalized bistatic phase patterns of specimen $\mathrm{L}$ at $6.17 \mathrm{GHz}$ (computed by use of the data in Table VI).

specimen I at $7.03 \mathrm{GHz}$, one may try other combinations different from the one chosen in Table IV. However, the E-plane amplitude measured at $\theta=135^{\circ}$ is greatly in error, as can be seen in Fig. 43, and should be avoided for any combination. Another natural way for improvement is to repeat and obtain better measured data for this specimen at $7.03 \mathrm{GHz}$. This can be done by our existing scattering range and receiver systems. On the other hand, the inaccuracy of 


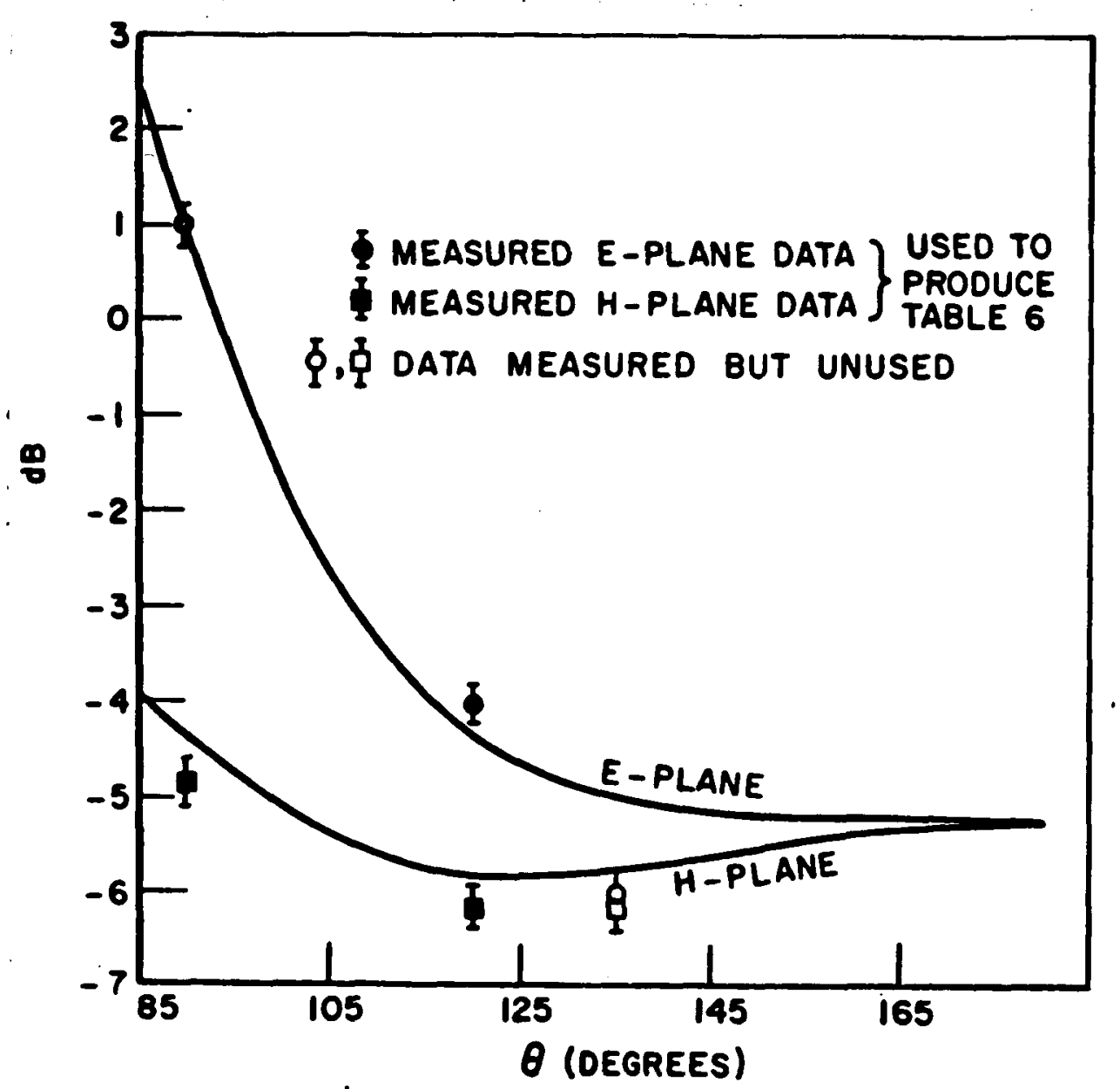

- Fig. 43--Normalized bistatic amplitude pattern of specimen I at $7.03 \mathrm{GHz}$ (computed by use of the nonconsistent results in Table $\mathrm{VI}$ ).

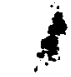

the parameters obtained for specimen $L$ at $7.03 \mathrm{GHz}$ cannot be improved by merely repeating our measurements. It is necessary in this particular case to reduce the sphere size so that the saddle point region can be avoided.
\end{abstract}




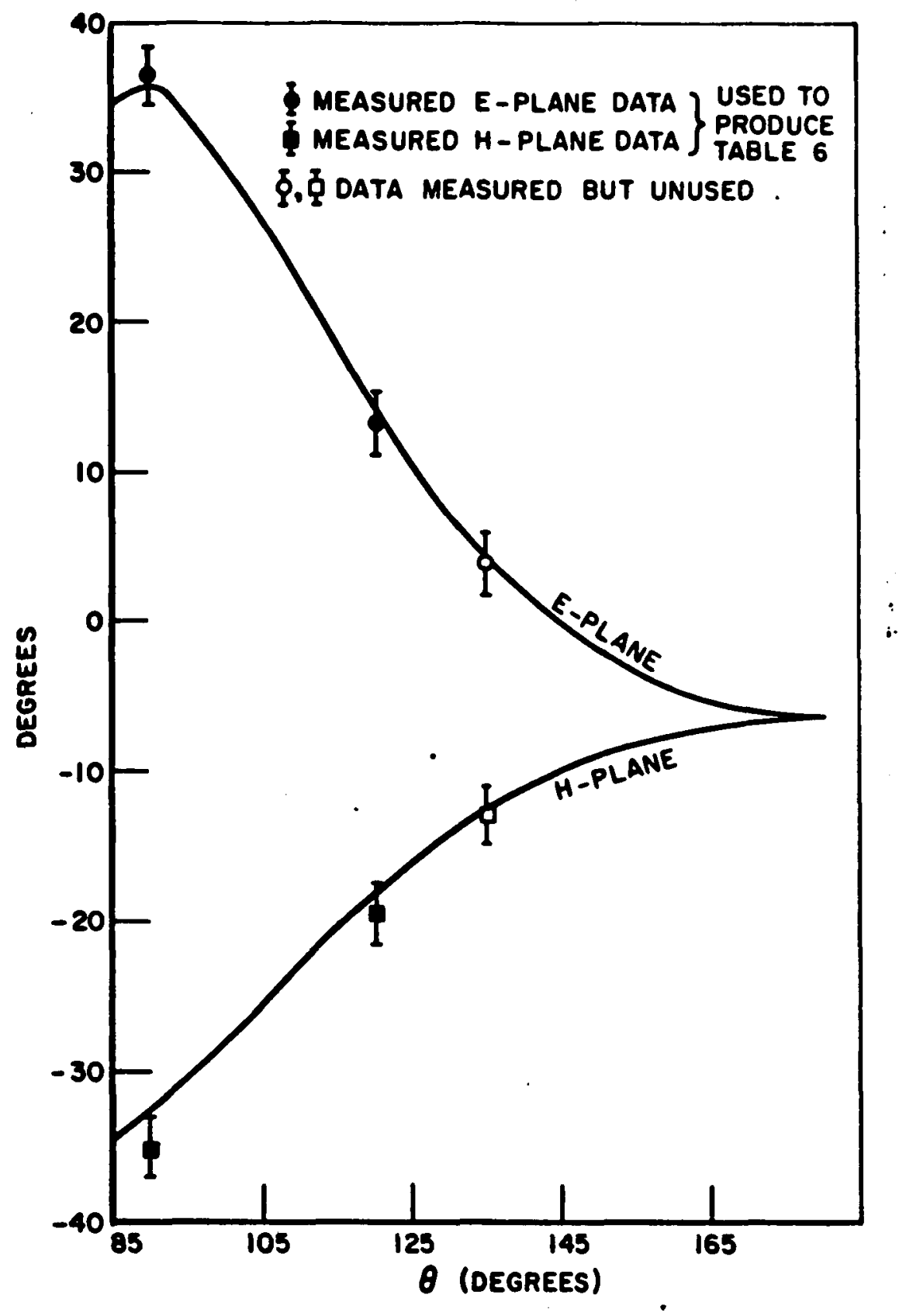

Fig. 44--Normalized bistatic phase pattern of specimen I at $7.03 \mathrm{GHz}$ (computed by use of the nonconsistent results in Table VI). 


\section{CHAPTER VI \\ NONUNIQUESNESS, PERTURBATIONS AND \\ RECOMMENDATIONS}

The problems of multiple roots in $X_{1}$ obtained from measured data are problems of nonuniqueness in inverse scattering. For a scattering sphere of given size, if there exists more than one set of material parameters $\left(\mu_{r}, \epsilon_{r}\right)$ or $\left(\eta_{r}, v_{r}\right)$ which would all give rise to the same scattered field within experimental accuracy over the entire scattering space, then these parameters are considered nonunique. Therefore, we shall discuss in this chapter the use of two specimen sizes to solve the problems of nonuniqueness.

\section{A. Two Specimen Sizes For A Unique Set of $\left(\mu_{r}, \epsilon_{r}\right)$}

Let us use a sphere size of $0.318 \lambda_{0}$ in diameter which corresponds to $p_{0}=\pi d / \lambda_{0}=1.0$. By referring to Fig. 9 for locations of individual refractive charts, one can use the three values of $v_{r} P_{0}$ identified as 1 , 2 and 3 in Table VII to show that they all have the same value of $\mathbf{X}_{1}=0.03+\mathrm{j} 0.98$. Together $w i$ th the value of $\eta_{r}$ given in the same Table, three sets of $\left(\mu_{r}, \epsilon_{r}\right)$ are obtained. Substitution of these parameters and po into Eq. (12) gives rise to three sets of theoretically distinct components of the scattered field. However, as shown in 
TABLE VII

THREE SETS OF $\left(\mu_{r}, \epsilon_{r}\right)$ USED TO ILLUSTRATE

"NONUNIQUENESS" AS SHOWN IN Fig. 45

\begin{tabular}{|l|l|c|c|c|c|c|}
\hline & $\left.\right|_{\mu_{Y}} \mid$ & $-\tan \delta_{\mu}$ & $\left.\right|_{\epsilon} \mid$ & $-\tan \delta_{\epsilon}$ & $\eta_{r}=\sqrt{\mu_{r} \epsilon_{r}}$ & $\rho_{0} \nu_{r}=\sqrt{\mu_{r} \epsilon_{r}} \rho_{O}$ \\
\hline 1 & 0.9972 & 1.102 & 23.46 & 0.358 & $0.2-j 0.05$ & $4.02-j 2.69$ \\
\hline 2 & 1.238 & 0.895 & 29.12 & 0.245 & $0.2-j 0.05$ & $5.31-j 2.80$ \\
\hline 3 & 1.846 & 0.536 & 43.42 & 0.0022 & $0.2-j 0.05$ & $8.68-j 2.19$ \\
\hline
\end{tabular}

$p_{0}=\pi d / \lambda_{0}=1.0$ or $d=0.318 \lambda_{0}$

$x_{1}=0.03+j 0.98$ 
Fig. 45, these scattered field components differ by no more than $0.1 \mathrm{~dB}$ in amplitude and agree to within $1^{\bullet}$ in phase angles. In other words, they agree within the limits of experimental accuracy. Consequently, we are not able to distinguish these three specimen spheres of different parameters $\left(\mu_{r}, e_{r}\right)$. The solution to this difficulty is to reduce the sphere size so that they would exhibit three different scattering characteristics. If $p_{0}$ is reduced to 0.5 , the subsequent amplitude patterns are shown in Fig. 46. Their phase patterns differ among themselves in the order of $10^{\circ}$ and are not plotted. Evidently, we are now able to distinguish these three specimens by use of our existing measurement systems.

Suppose we were able to obtain from measurements on one of those three larger specimens the values of $\left(\eta_{r}, X_{1}\right)=(0.2-j 0.05$, $0.03+j 0.98)$. This value of $x_{1}$ would give all together four appropriate roots, the first three roots $\nu_{r} p_{O}$ are given in Table VII and the largest one is at $v_{r} \rho_{0}=11.9-j 2.08$. For simplicity, we assume the largest root can be excluded from consideration. The problem now is to decide which one of the three sets of $\left(\mu_{r}, e_{r}\right)$ in Table VII is the correct one to characterize the sphere being considered here. If this particular specimen were measured under the reduced size $p_{0}=$ 0.5, it would exhibit only one of the three patterns shown in Fig. 46. These patterns would produce three distinct values of $X_{1}$ as 


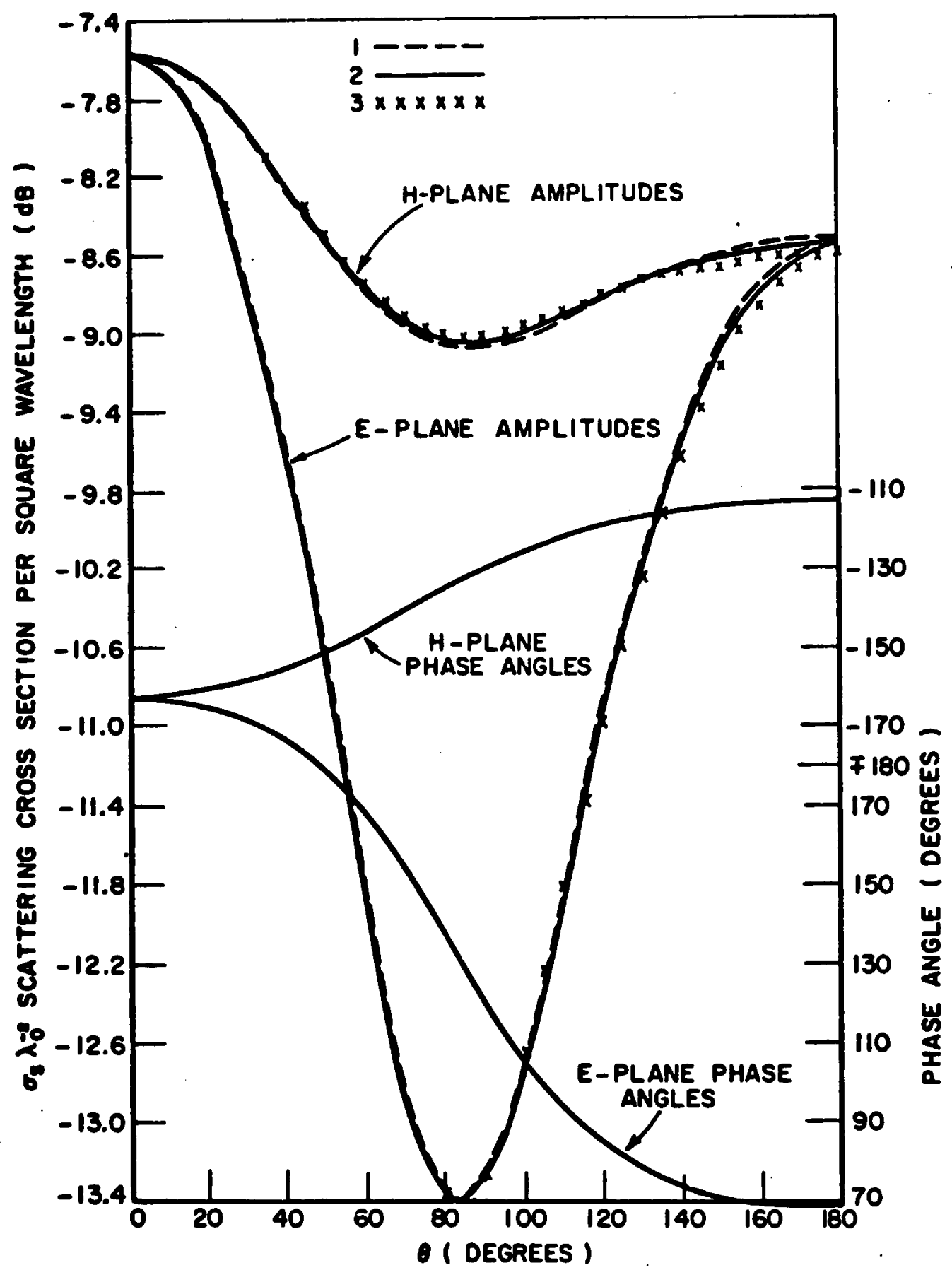

Fig. 45-- Three bistatic scattering patterns, produced by the three sets of $\left(\mu_{r}, e r\right)$ in Table VII, differ among themselves by less than $0.1 \mathrm{~dB}$ and $1^{\circ}$. 


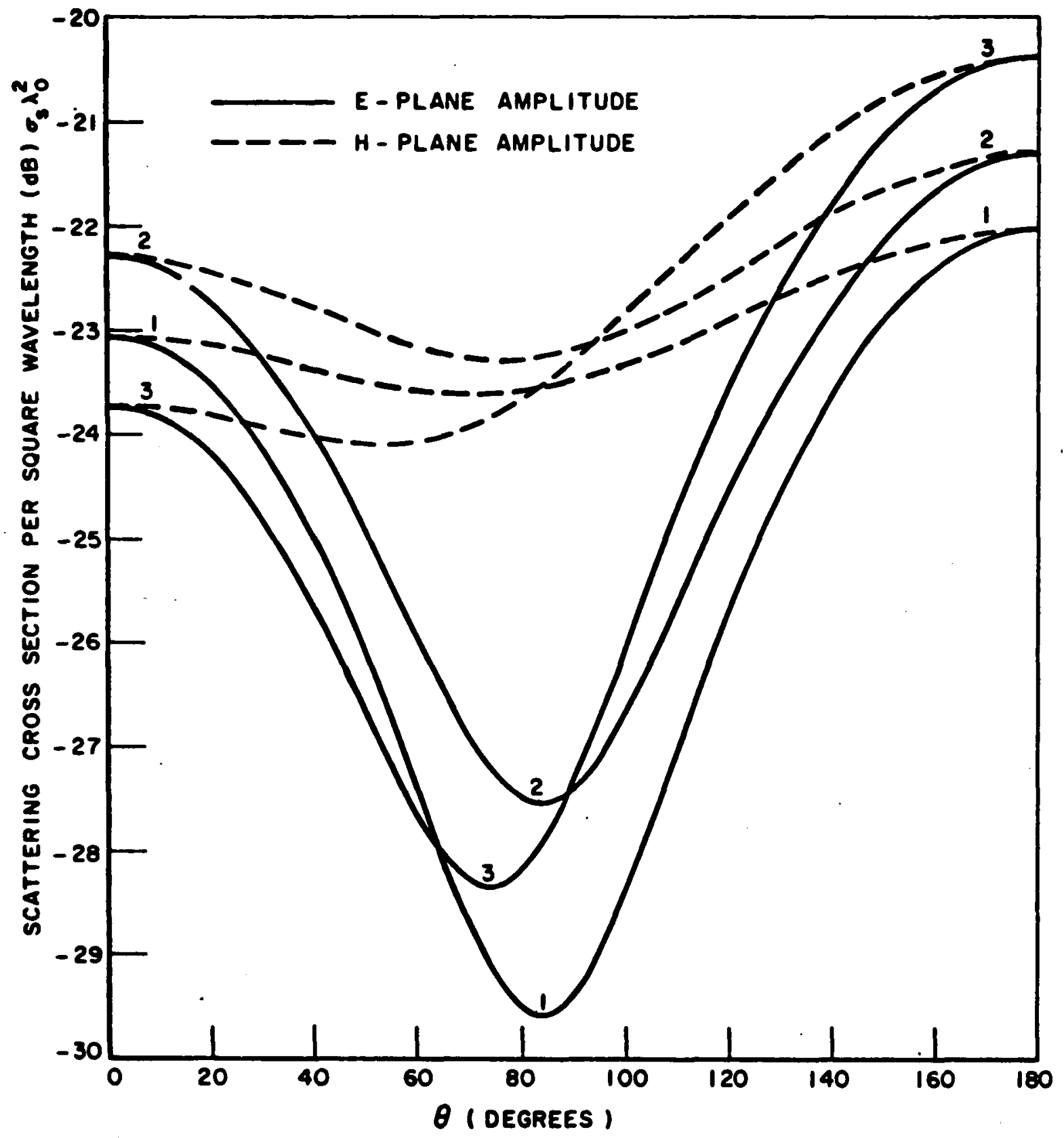

Fig. 46--Amplitude patterns of the three specimens, characterized by the $s$ ame parameters $\left(\mu_{r}, \epsilon_{r}\right)$ used for Fig. 45, when their size is reduced to $p_{0}=0.5$. 


$$
\begin{aligned}
& \left(x_{1}\right)_{1}=0.324+j 0.806 \\
& \left(x_{1}\right)_{2}=0.112+j 0.764
\end{aligned}
$$

and

$$
\left(X_{1}\right)_{3}=-0.0757+j 1.205 .
$$

They would in turn give rise to three distinct roots $v_{r} \rho_{O}$ as

$$
\begin{aligned}
& \left(v_{x} \rho_{0}\right)_{1}=2.01-j 1.395, \\
& \left(v_{x} \rho_{0}\right)_{2}=2.66-j 1.40, \\
& \left(v_{x} \rho_{0}\right)_{3}=4.34-j 1.095,
\end{aligned}
$$

where

$$
P_{0}=0.5
$$

If the sphere being considered here, under the reduced size, exhibited the number 3 pattern in Fig. 46, then the third root given above belongs to this specimen sphere. Dividing this root by $p_{0}=0.5$ gives $v_{r}=8.68-\mathrm{j} 2.19$ which together with $\eta_{r}=0.2-\mathrm{j} 0.05$ give the number 3 set of the parameters $\left(\mu_{\Gamma}, \epsilon_{r}\right)$ in Table VII. It is in this sense that our scattering method is capable of determining $\left(\mu_{r}, \epsilon_{r}\right)$ of a specimen uniquely.

B. Perturbations Of Scattered Fields By The Parameters

We next tirn our attention to perturbations of scattered fields that would be caused by certain amounts of change in material parameters $\left(\mu_{Y}, \epsilon_{r}\right)$. The purpose of this investigation is to analyze the limitations of our scattering method, in regard to the lossiness of 
media, the size of specimens and the accuracy required of measurement systems.

The expressions in Eq. (12) indicate that, excluding $s_{o}$, there are four independent parameters in far-zone scattered field components. Perturbations that could be caused by the parameters $\left(p_{0}, \theta\right)$ of specimen sphere are in the same order of amounts as shown in Figs. 26 and 27 for a conducting sphere. Here we are to hold the size $p_{0}$ of a specimen constant, and study effects of changes by $\left(\mu_{r}, e_{r}\right)$ over the range of the bistatic angle $85 \leq \theta \leq 180^{\circ}$. These specimens of the same size are shown in Table VIII. Their parameters $\left(\mu_{r}, \epsilon_{r}\right)$ are to be changed from their correct values to the two representative values on a $5 \%$-circle shown in Fig. 47 for the parameter $\mu_{r}$. The notations used in this figure are to be identified with those used in Figs. 48 to 59 . The perturbations indicated in these error patterns are numerical deviations of the scattered fields from those before the 5\%-changes were made. For instance, if $\mathrm{E}$ is a scattered field amplitude by use of the parameters in Table VIII, and if $1.02 \mathrm{E}$ is the corresponding scattered field amplitude due to a 5\%-change in either $\mu_{r}$ or $\epsilon_{r}$, then an error of $0.172 \mathrm{~dB}=20 \log (1.02)$ would represent a $2 \%$-increase in field amplitude.

In Fig. 48, a change from $\mu_{Y}$ to $0.95 \mu_{x}$ causes a large E-plane phase error in the region near $\theta=90^{\circ}$. This error decreases gradually to $0.5^{\circ}$ at $\theta=180^{\circ}$. The same change in $\mu r$ produces $H$-plane phase error from $0.1^{\circ}$ at $\theta=85^{\circ}$ to $0.5^{\circ}$ at $\theta=180^{\circ}$. Comparisons in these 
TABLE VIII

THREE SETS OF $\mu_{r}$ AND \& $r$ WITH 5\% CHANGES

INTRODUCED TO STUDY THE CONSEQUENT

CHANGES IN SCATTERED FIELDS

\begin{tabular}{|l|l|l|l|}
\cline { 2 - 4 } \multicolumn{1}{c|}{} & \multicolumn{1}{|c|}{1} & \multicolumn{1}{c|}{ Specimen } & \multicolumn{1}{c|}{3} \\
\hline$\left.\right|_{\mu_{r}} \mid$ & 1.005 & 1.959 & 1.831 \\
\hline$-\tan \delta_{\mu}$ & 0.0012 & 0.517 & 1.11 \\
\hline$\epsilon_{r} \mid$ & 2.525 & 11.07 & 17.0 \\
\hline$-\tan \delta_{C}$ & 0.008 & 0.015 & 0.063 \\
\hline$\eta_{r}$ & $0.6308+j 0.00212$ & $0.4095-j 0.0962$ & $0.3038-j 0.1241$ \\
\hline$\nu_{r} \rho_{O}$ & $1.308-j 0.006$ & $3.71-j 0.93$ & $4.125-j 1.995$ \\
\hline$x_{1}\left(\nu_{r} \rho_{O}\right)$ & $1.255-j 0.01$ & $-0.325+j 0.905$ & $0.005+j 0.992$ \\
\hline
\end{tabular}

$P_{0}=0.82112$

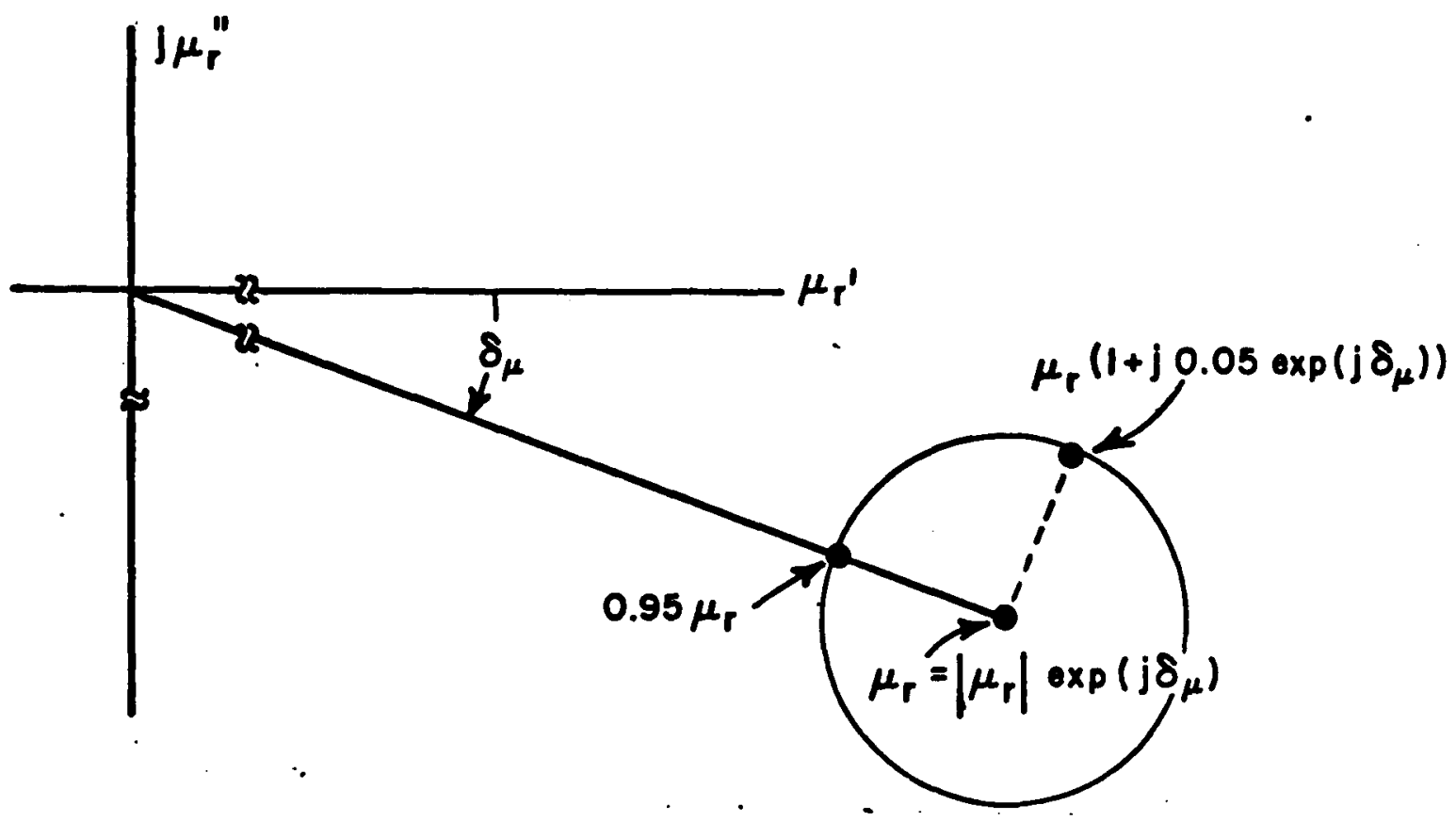

Fig. 47--Two representative values on the 5\%-circle of the parameter $\mu_{\mathbf{r}}$. 


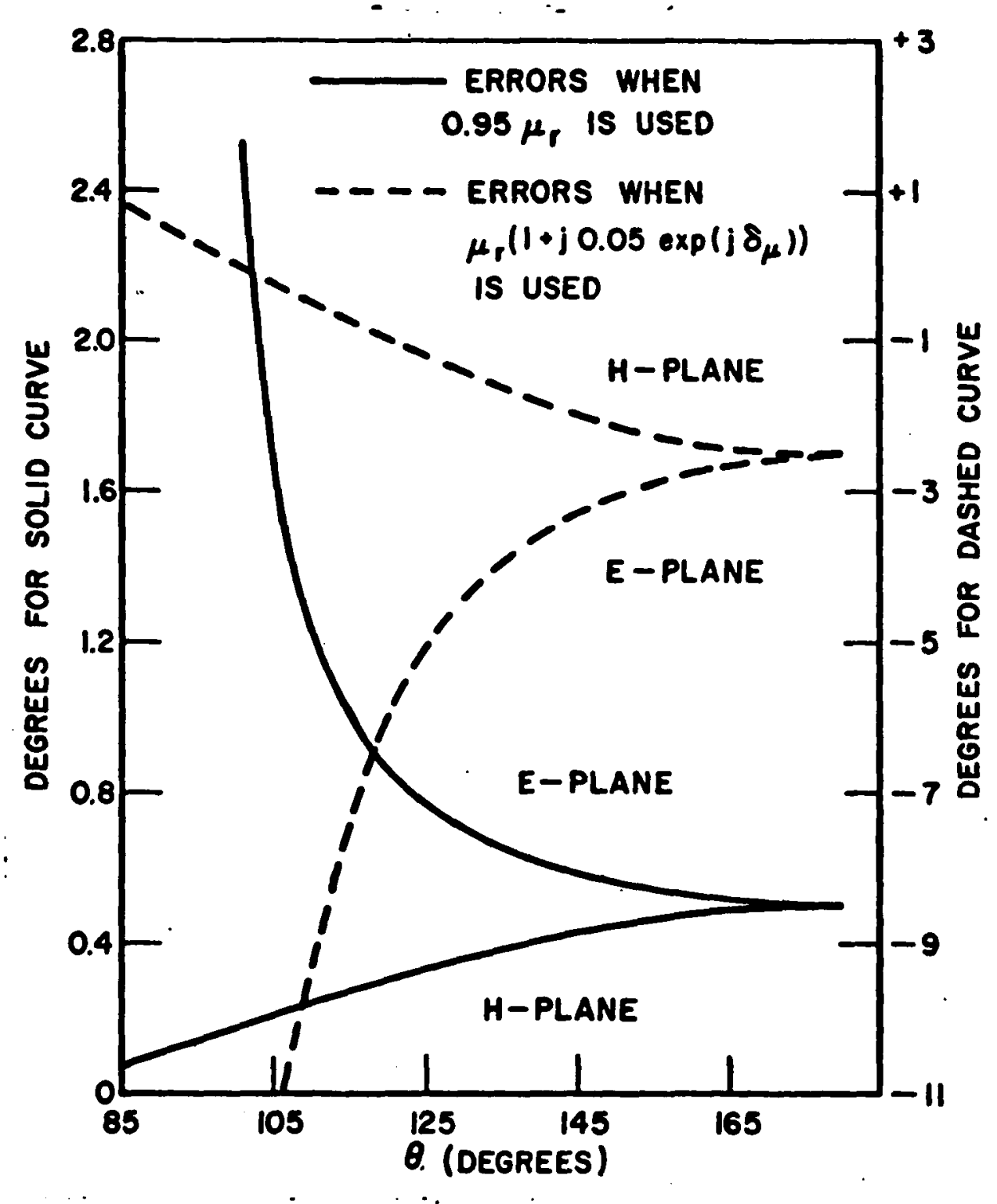

Fig. 48--Phase error patterns due to $5 \%$-changes in $\mu_{r}$ of specimen 1 in Table VIII. 


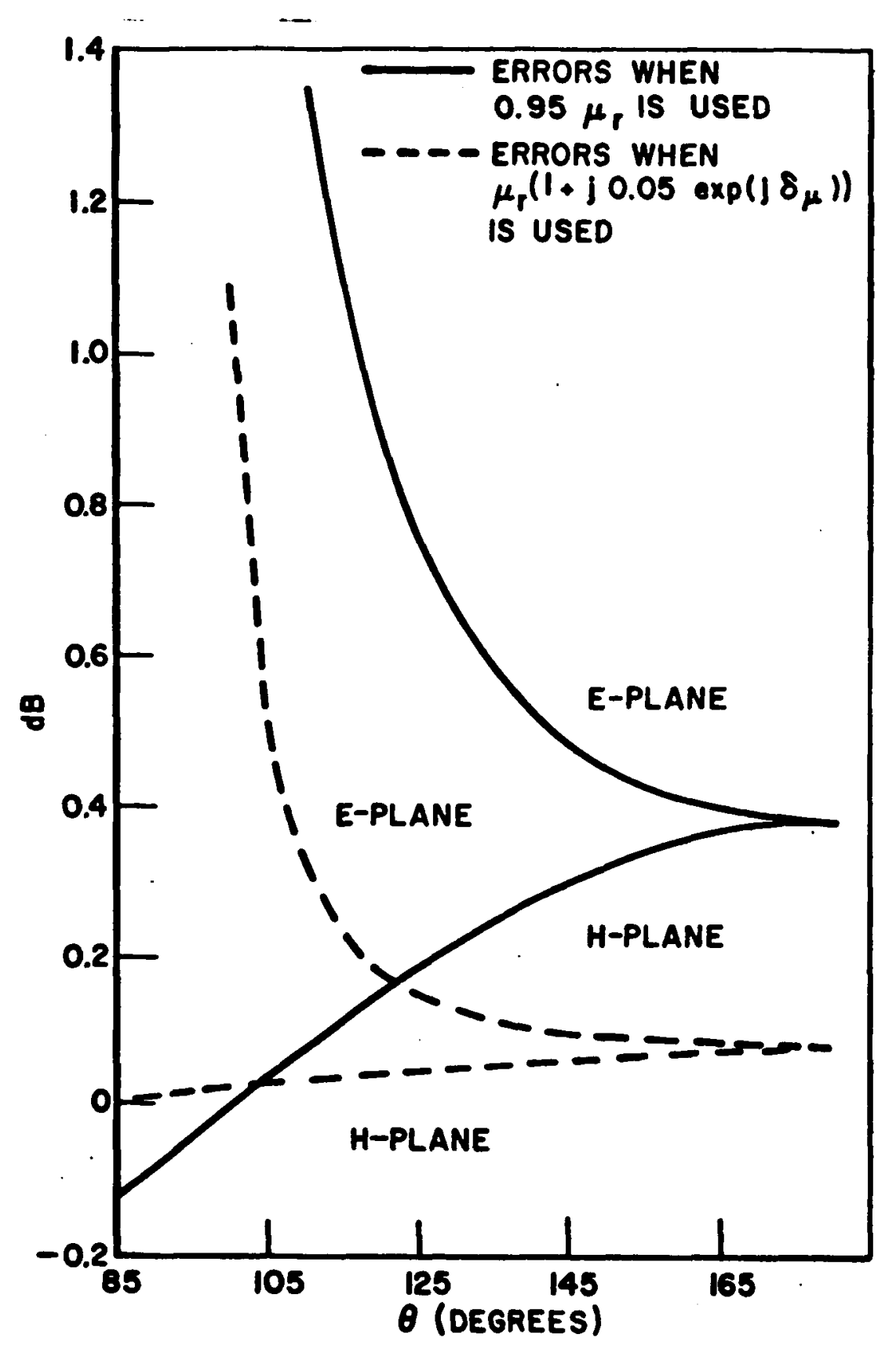

Fig. 49--Amplitude error patterns due to 5\%-changes in $\mu_{r}$ of specimen 1 in Table VIII. 


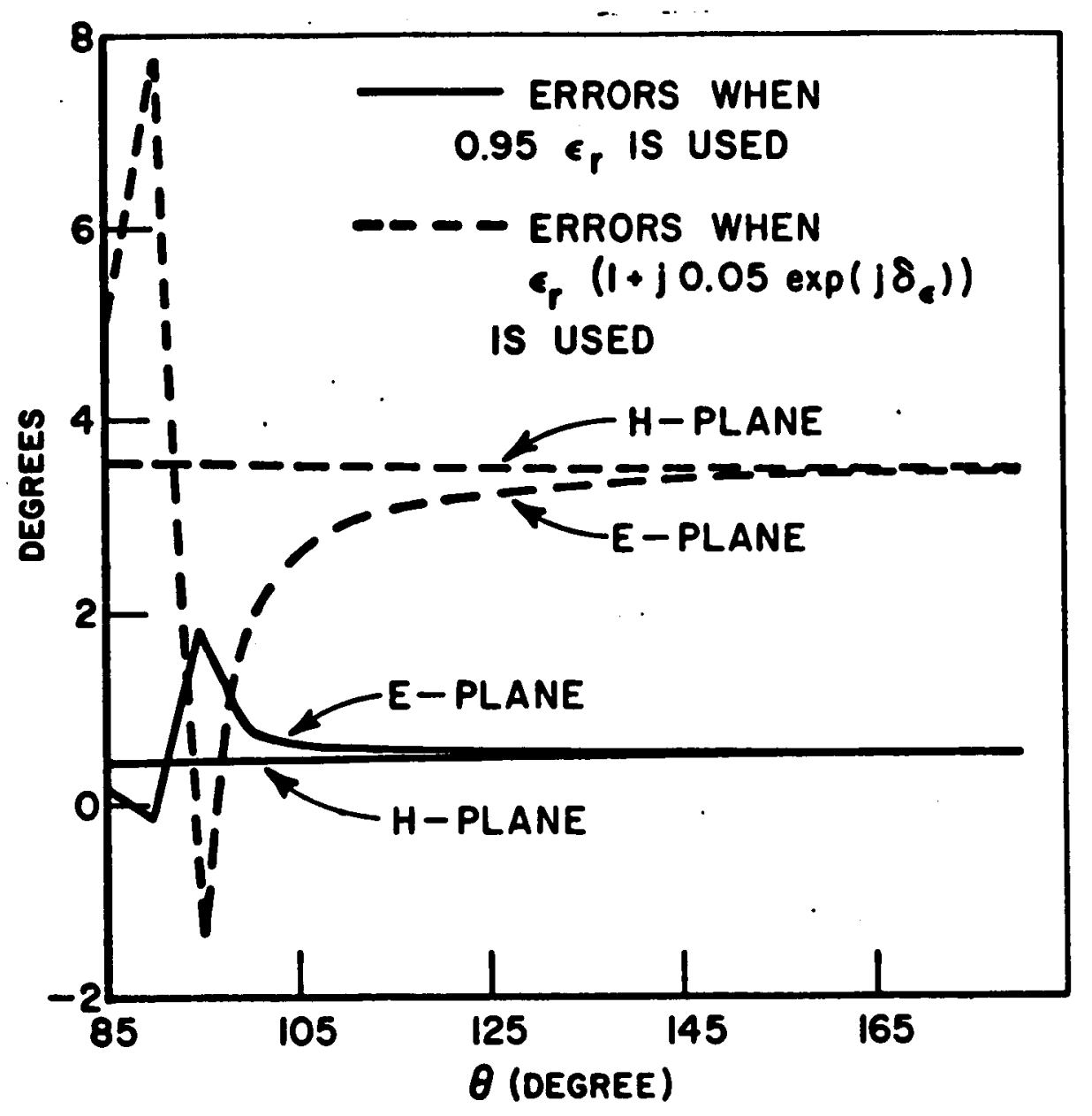

Fig. 50--Phase error patterns due to 5\%-changes in er of specimen $l$ in Table VIII.

two phase error patterns indicate that the E-plane phase angle is more sensitive to a change in the magnitdue of $\mu_{r}$ than the $H$-plane phase angle. This implies that, if one were required to determine $\mu_{r}$ accurate to $5 \%$ of its true value, measured phase errors in the E-plane is allowed to be larger than $0.5^{\circ}$, but less than $0.5^{\circ}$ is restricted to the H-plane phase errors. Similar observations can be made to the dashed patterns which result from a change in phase angle of $\mu_{r}$ as shown in Fig. 47. In case the value of $\mu_{r}$ listed in Table VIII is 


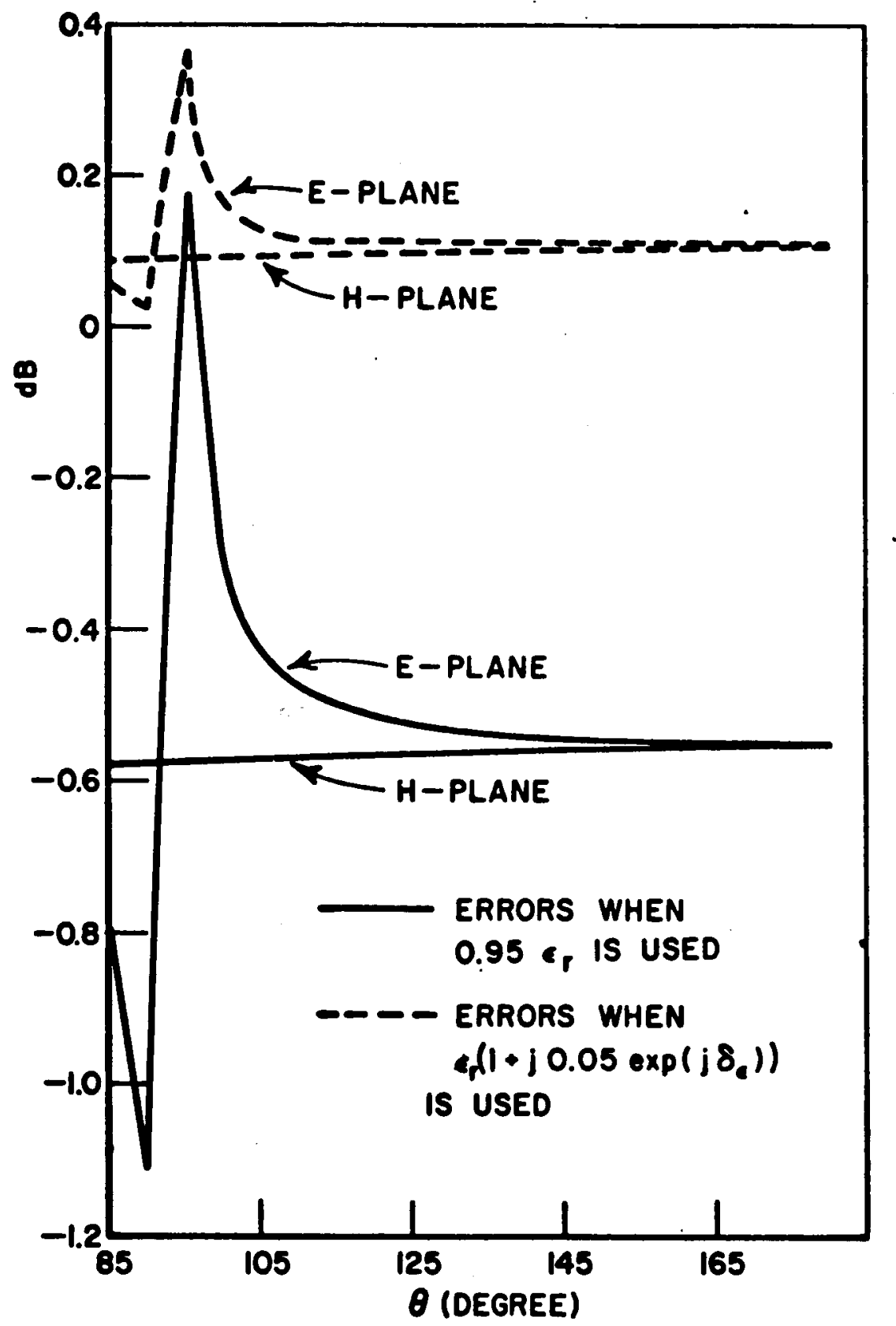

Fig. 51--Amplitude error patterns due to 5\%-changes in $\epsilon_{r}$ of specimen 1 in Table VIII. 


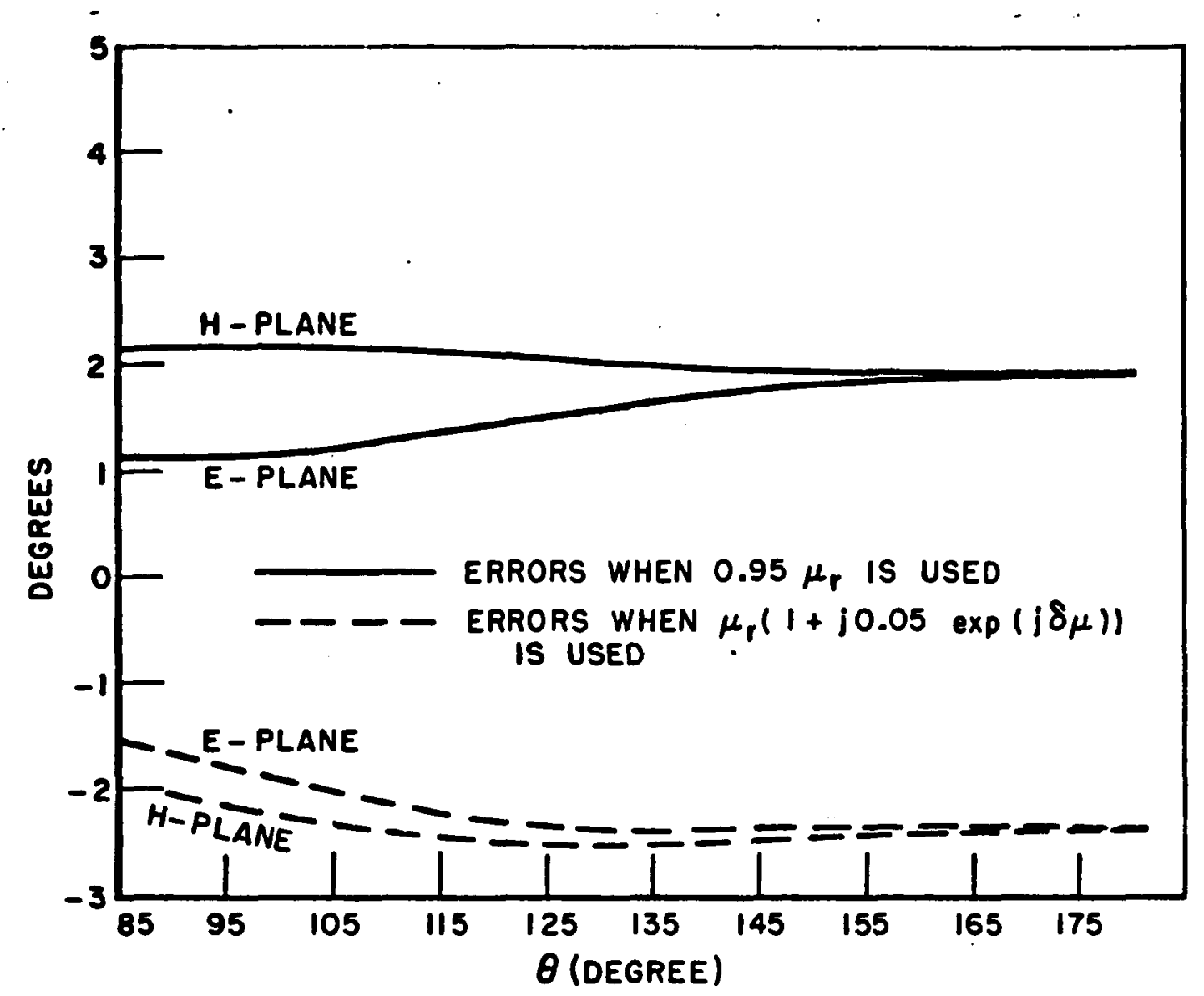

Fig. 52--Phase error patterns due to 5\%-changes in $\mu_{r}$ of specimen 2 in Table VIII.

uncertain in its accuracy, and the scattering measurements need be repeated and improved to obtain better accuracy for $\mu_{r}$, then informations contained in Fig. 48 are helpful in deciding what kinds of measurement accuracy are required specifically at different bistatic angle $\theta$. Similarly, Figs. 48 to 59 all can be used for this purpose, if their parameters are all uncertain in accuracy.

We note that the error patterns of discontinuous slope in Figs. 50 and 51 are due to insufficient data. They are plotted for each $5^{\circ}$ in $\theta$ and each two immediate neighboring points are joined by a straight 


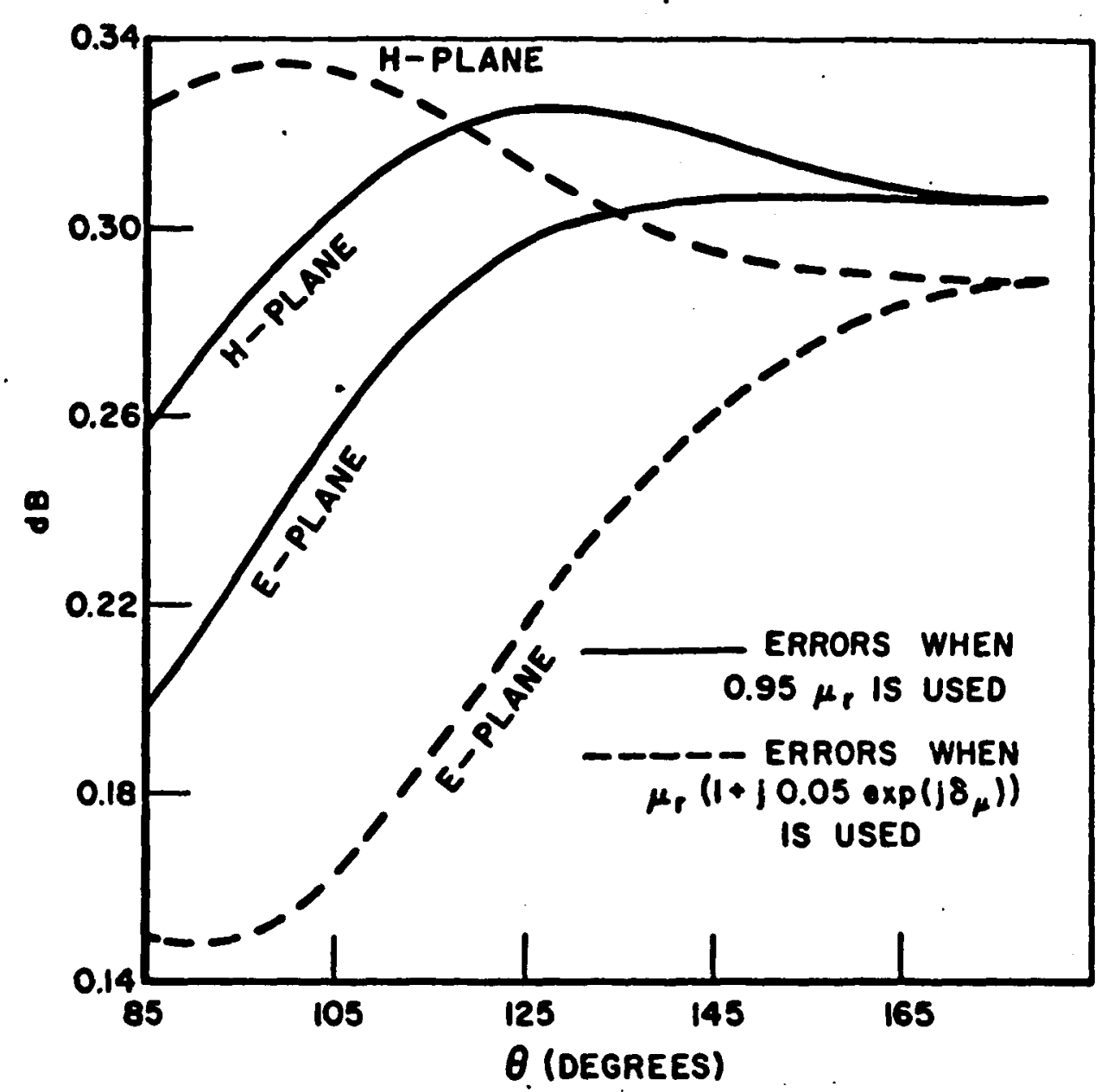

- Fig. 53--Amplitude error patterns due to 5\%-changes in of specimen 2 in Table VIII.

line. In Figs. 52 to 55, error patterns for a moderately lossy specimen are seen to have generally smaller perturbations than those of the low loss specimen in Figs. 48 to 51 . For the most lossy specimen, whose error patterns are shown in Figs. 56 to 59, we see that the perturbations to scattered field become smaller than $0.1 \mathrm{~dB}$ in amplitudes and $1^{\circ}$ in phase angles, and it is cleax that $5 \%$ accuracy could not be achieved in evaluating its constitutive parameters. 


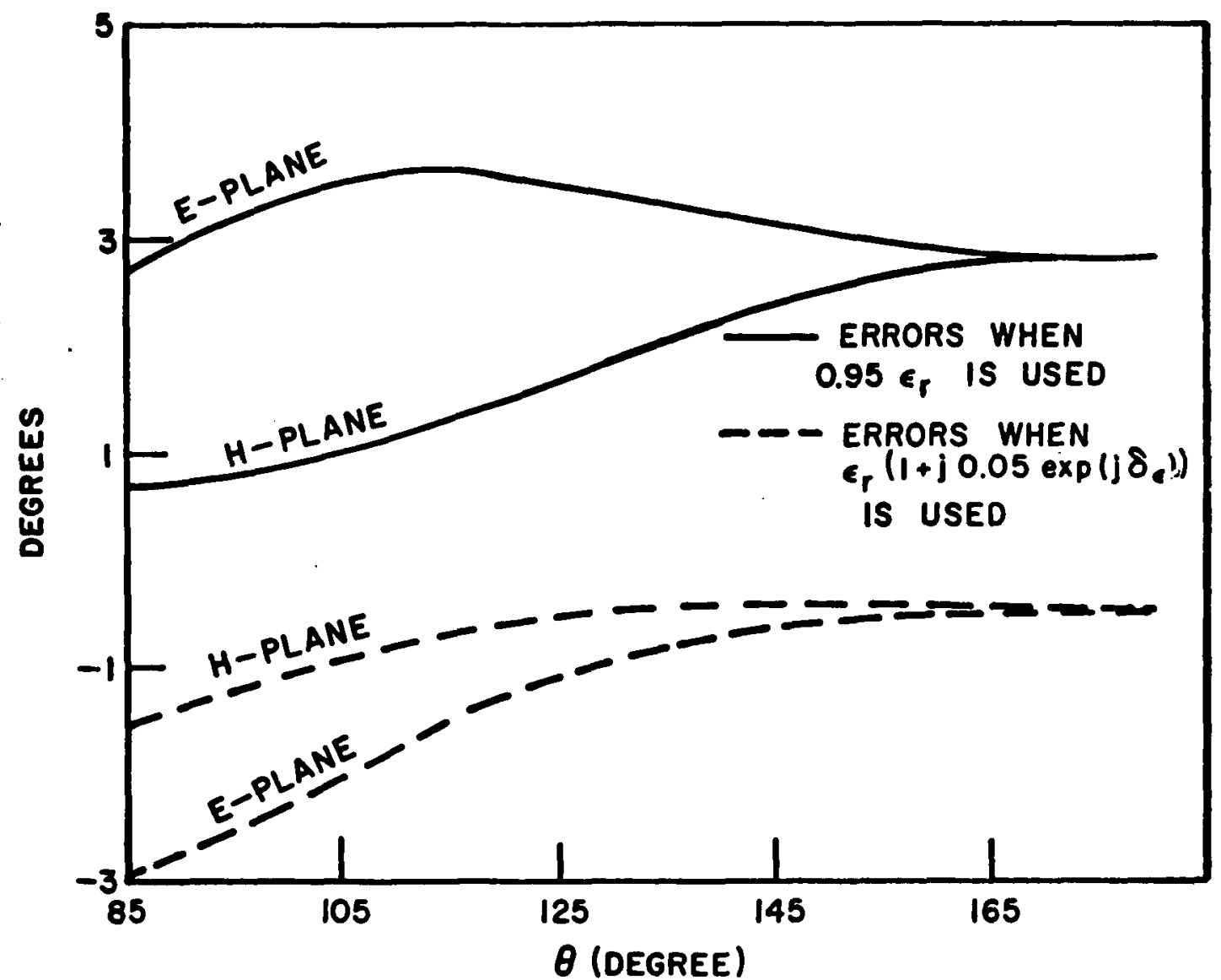

Fig. 54--Phase error patterns due to 5\%-changes in $\mathrm{er}$ of specimen 2 in Table VIII.

The error patterns of the most lossy specimen reveal the limitations on lossiness of a material which can be effectively measured by the present method. So long as no measurement systems with less than $0.1 \mathrm{~dB}$ and $1^{\bullet}$ uncertainties are available, no accurate parameters can be obtained for specimen spheres whose sizes and losses are such that $v_{r} " \rho_{0}<-3$ in the refractive index chart. Under these circumstances specimen sizes need be reduced to improve parameters accuracy. 


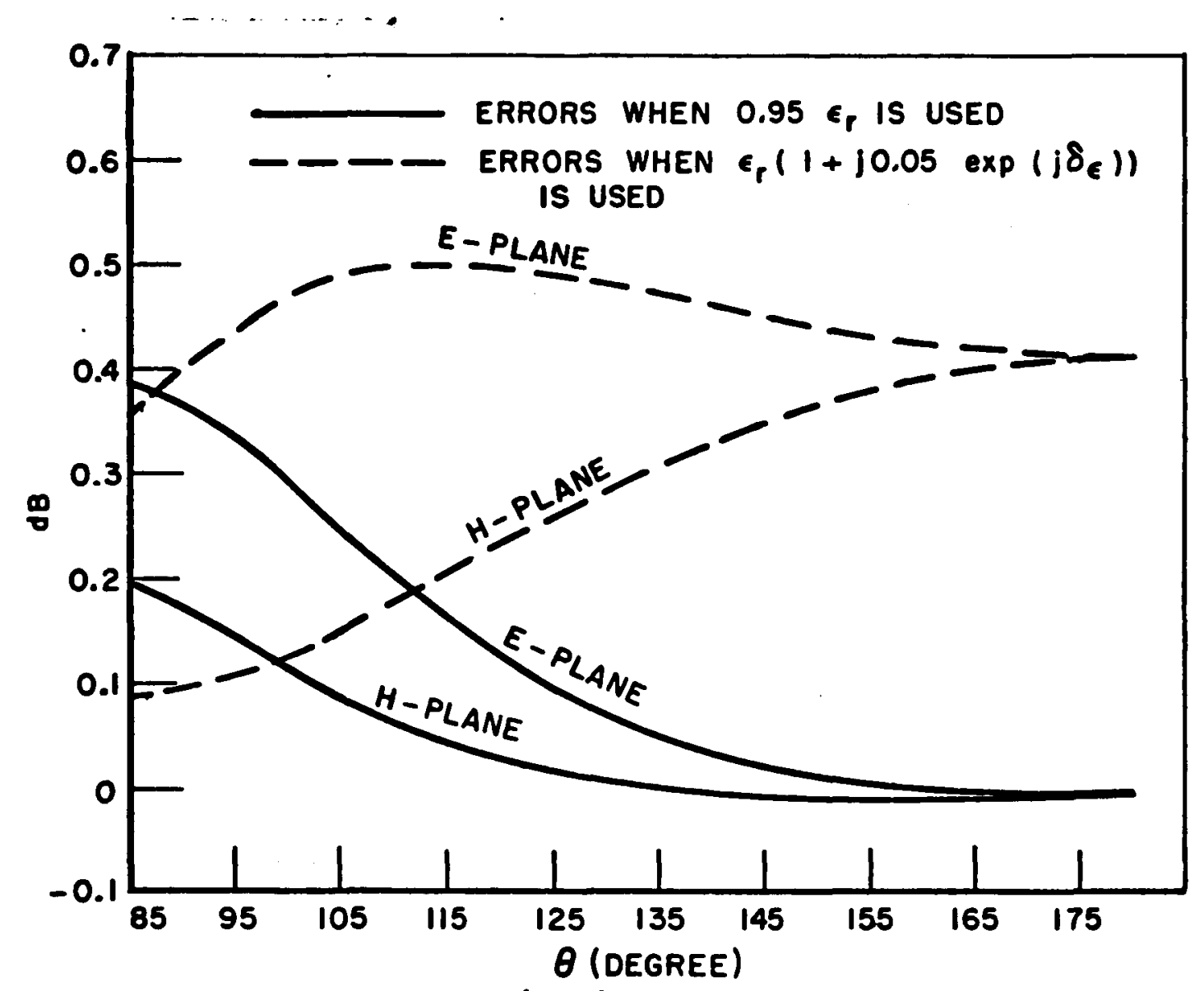

Fig. 55--Amplitude error patterns due to $5 \%$-changes in $\epsilon_{r}$ of specimen 2 in Table VIII.

\section{Recommendations}

Since the accuracy of determining the constitutive parameters depends on the measurement accuracy, improvements in the actual measured data are of prime importance. Fortunately there are several improvements that should be possible. Perhaps the most important advance would be the design of a system to measure the phase of the scattered signal with greater accuracy. A realistic and complete study of the phase measurement techniques is beyond the scope of this present 


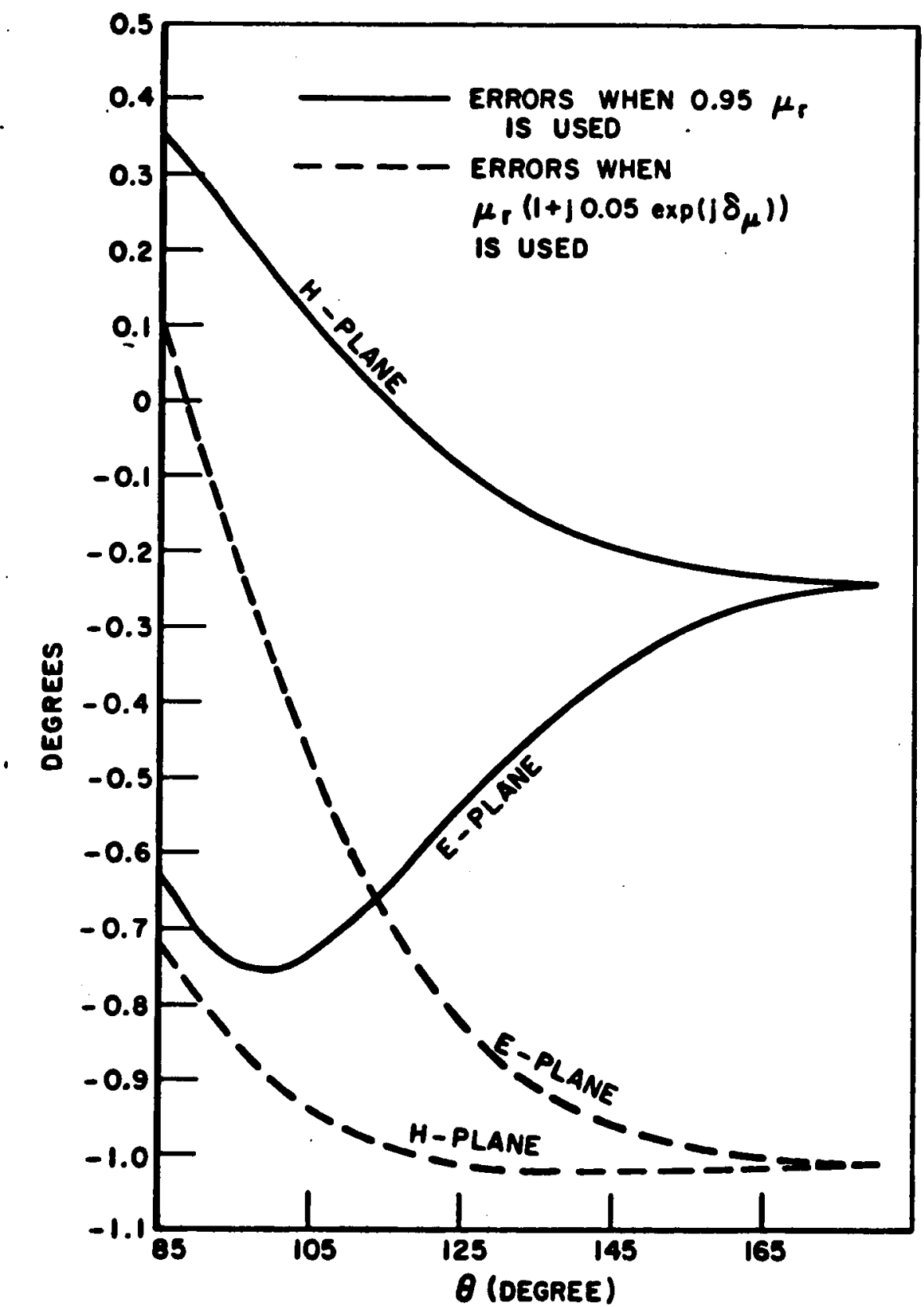

Fig. 56--Phase error patterns due to 5\%-changes in $\mu_{r}$ of specimen 3 in Table VIII. 


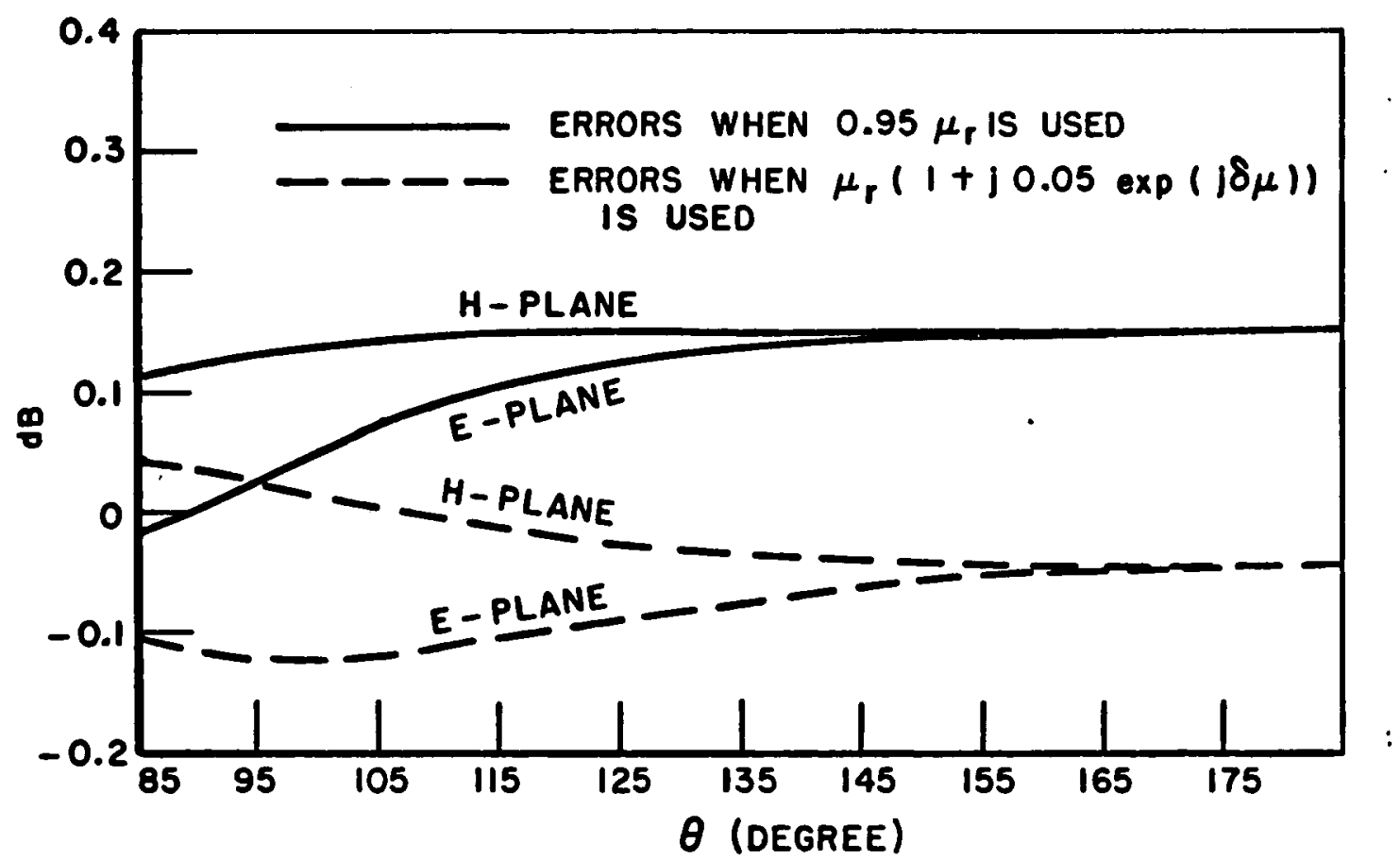

- Fig. 57--Amplitude error patterns due to 5\%-changes is $\mu_{r}$ of specimen 3 in Table VIII.

investigation. The phase receiver available was used to obtain data needed to demonstrate the concepts discussed in the present method. A second improvement in the measured data would require making a sufficiently large number of scattered field measurements at each bistatic angle to invoke statistical techniques such as the central limit theorem.[18] This would make it possible to substantially reduce all random errors and would effectively reduce the null level of the radar system. Spentzos [12] demonstrated crudely the advantage of repeated measurements for this purpose. This has not been pursued in the present study because of the time involved. However there is no doubt 


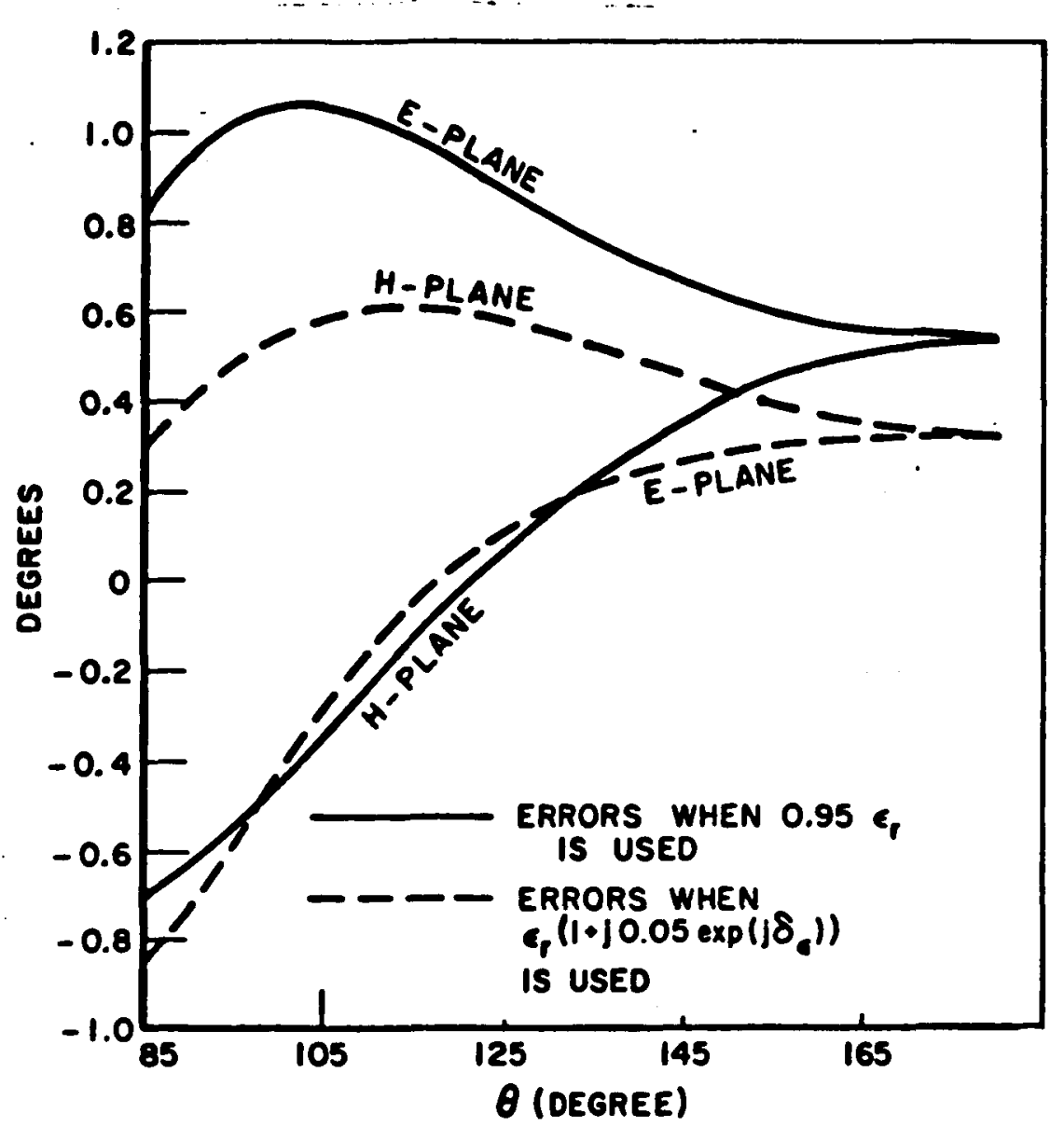

Fig. 58--Phase error patterns due to 5\%-changes in $\epsilon_{r}$ of specimen 3 in Table VIII.

that truly random errors could be reduced in this way. Instead of this time-consuming process, the initial adjustments have been used in this investigation to obtain a reasonably consistent solution before proceding to the consistency process. Finally measurements could be made at a much larger number of bistatic angles. Then $\left(a_{1}, b_{1}, a_{2}, b_{2}\right)$ could be obtained such that a mean square fit is obtained for the measured $y^{\prime} s$ and the bistatic patterns are obtained from these coefficients. To illustrate this mean square fit we substitute Eq. (10) into Eq. (15) to obtain 


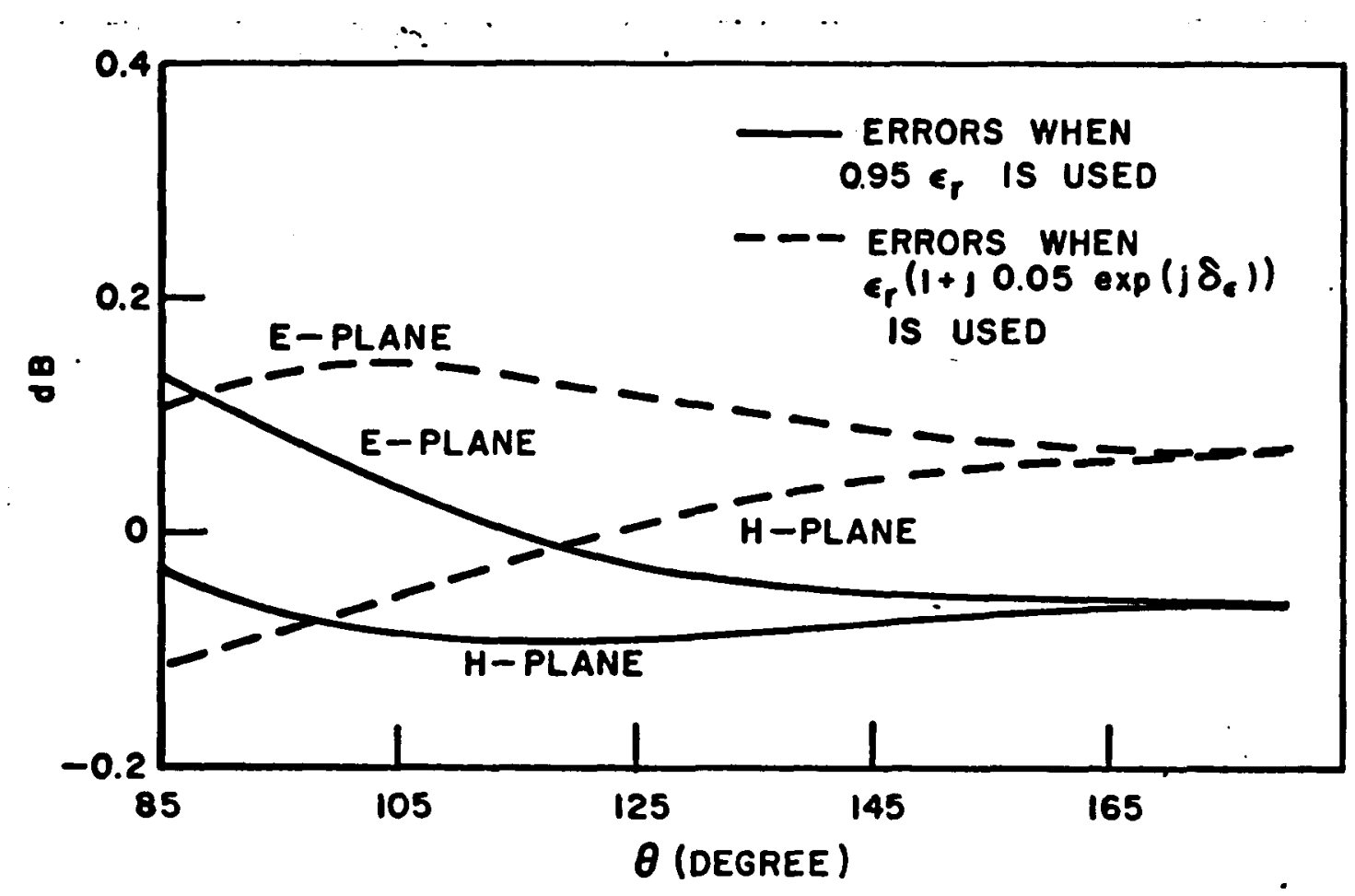

Fig. 59--Amplitude error patterns due to 5\%-changes in $\epsilon_{r}$ of specimen 3 in Table VIII.

$$
\begin{aligned}
3 a_{1} & +\left(3 b_{1}+5 a_{2}\right) \cos \theta_{1}+5 b_{2} \cos 2 \theta_{i}= \\
& \gamma \theta\left(\theta_{i}\right)\left[3 a_{1}^{c}+\left(3 b_{1}^{c}+5 a_{2}^{c}\right) \cos \theta_{i}+5 b_{2}^{c} \cos 2 \theta_{i}\right],
\end{aligned}
$$

and

$$
\begin{aligned}
3 b_{1}+ & \left(3 a_{1}+5 b_{2}\right) \cos \theta_{i}+5 a_{2} \cos 2 \theta_{i}= \\
& \gamma_{\phi}\left(\theta_{i}\right)\left[3 b_{1}^{c}+\left(3 a_{1}^{c}+5 b_{2}\right) \cos \theta_{i}+5 a_{2}^{c} \cos 2 \theta_{i}\right] .
\end{aligned}
$$

When measurements at $\theta_{i}$ are made the $r$ ight hand sides of the above expressions are constant and we designate them as $C_{i}$ and $D_{i}$, respectively, corresponding to $\theta_{\mathfrak{i}}$. Also using summation notations for the left hand sides we rewrite 


$$
\sum_{m=0}^{2} c_{m} \cos m \theta_{i}=c_{i}
$$

and

$$
\sum_{m=0}^{2} d_{m} \cos m \theta_{i}=D_{i}
$$

Since errors are involved in the measured quantities $C_{i}$ and $D_{i}$, the sums of the squares of errors are written as

$$
F=\sum_{i=1}^{N}\left[c_{i}-\sum_{m=0}^{2} c_{m} \cos m \theta_{i}\right]^{2},
$$

and

$$
G=\sum_{i=1}^{N}\left[D_{i}-\sum_{m=0}^{2} d_{m} \cos m \theta_{i}\right]^{2}
$$

By making $\partial F / \partial c_{m}=0$ and $\partial G / \partial d_{m}=0$ for $m=0$ to 2 , each of these result in three simultaneous linear equations to solve for $\bar{c}_{m}$ and $\bar{d}_{m}$. the estimates of $c_{m}$ and $d_{m}$. Thus estimates for $\left(a_{1}, b_{1}\right)$ and $\left(a_{2}, b_{2}\right)$ may be obtained.

At this stage, the consistency process would be introduced in the same fashion as developed in the text of this investigation. Confidence in the values of the constitutive parameters so obtained would be substantially enhanced. This becomes even more evident since the spread 
of the measured data would be considerably reduced and it would become much more demanding to fit the measured data to the patterns computed from the final values of the constitutive parameters.

If sufficient precision can be achieved it would be possible to employ an "octupole sphere". This would make it possible to increase the radius of the sphere and the larger scattered fields would tend to improve the measurement accuracy. Such a step would involve a third pair of mode coefficients $\left(a_{3}, b_{3}\right)$. In this case, the solutions $\eta_{r}\left(a_{1}, b_{1}\right)$ and $\eta_{r}\left(a_{2}, b_{2}\right)$ can be required to satisfy a $10 \%$ consistency criterion. Similarly, $X_{2}$ would be more accurate than that obtainable from the use of a quadrupole sphere, and the consistency criterion for $\nu_{r} \rho_{0}$ could also be reduced to about $10 \%$. These criteria are estimated by solutions obtained from exact bistatic fields .

Finally it is noted that all of these procedures have been developed for a sphere of a fixed radius. New information can be obtained if a set of specimen spheres of different radii are investigated. It is apparent that the exact values of $v_{\mathbf{r}} p_{\mathrm{O}}$ plotted on the refractive index chart as a function of radius would yield a straight line. If the values of $v_{x} p_{0}$ obtained from measured data were also so plotted, a best fit straight line would yield the most accurate value for the complex refractive index. Meanwhile the intrinsic impedance of the same specimen spheres would be independent of the different radii used. Thus another degree of freedom can be added to the measured results 
to further enhance the accuracy of the end results. Spheres of different radius can also be used in another way to further increase the accuracy of the techniques once a good value for the constitutive parameters has been obtained. This may require a larger sphere than has been considered here. Assume, for example, that $\mu_{x}=1$, the value of e $_{r}$ ' is known with good accuracy but er" has not been determined with sufficient accuracy. Then a sphere can be found by trial and error for which the scattered field at some bistatic angle is dependent most on the value of $e^{\prime \prime}$. By use of the refractive index chart, measurement of the scattered field for a sphere of this radius would then more precisely establish $\boldsymbol{c r}^{\prime \prime}$. 


\section{CHAPTER VII}

CONCLUSIONS

A method of determining the constitutive parameters has been developed by measuring the scattered field components of a specimen sphere. This method uses a newly constructed refractive index chart to find the refractive index of a specimen. A consistency process based on the Mie solution has been employed to reduce the uncertainties caused by theoretical approximations and experimental inaccuracies. The final results obtained for a cross-linked polystyrene specimen have indicated that the present method is capable of producing accurate parameters. The final results obtained for a moderately lossy and two highly lossy specimens have also demonstrated the usefulness of this method for determining loss parameters.

By considering higher-order scattering coefficients of a specimen, this method allows relatively large specimen size as compared to conventional methods, which consider only the lowest order modes resulting from boundary conditions. Measurements using different sizes of a specimen to assure uniqueness and to improve accuracy of final results are recommended. Lossy specimens can always be effectively measured by this method, provided that they can be considered as penetrable spheres $\left(\nu_{r} " p_{0}>-3\right)$. 
A technique of target rotation has been used not only to determine the homogeneity and isotropicity of a specimen, but also to provide more meaningful phase angle measurements of the scattered fields. The target support used is simple and has no difficult problems of target fitting and positioning. As far as microwave measurements are concerned, the present method is considered to have the following useful features:

1. The scattering method can provide independent checks on results obtained by other methods. [5-8]

2. Specimens can be as large as $0.32(0.5)$ free space wavelength in diameter when quadrupole (octupole) type spheres are used. A specimen of different radii can thus be measured to improve the accuracy of final results.

3. Specimen fitting and positioning are relatively easy.

4. The homogeneity and isotropicity of a specimen can be qualitatively determined with ease by specimen rotation.

5. The method is useful for specimens of high losses and/or large refractive indices.

6. The method has certain versatility that measur ements can be made at different bistatic angles to obtain highly organized end results which must be consistent with the Mie solution.

It has a great disadvantage in that it requires a sophisticated scattering range and a time-consuming technique requiring application 
of a digital computer. It also suffers from a limitation common to other methods in that accurate phase measurements are required. The refractive index chart has been demonstrated in the Appendices as a useful tool in studying scattering characteristics of a small sphere with arbitrary media. By use of this chart the scattering coefficients and patterns of a sphere can be obtained with good accuracy without use of a digital computer for evaluation of spherical Bessel functions of a complex argument. 


\section{APPENDIX I \\ PROPERTIES OF A SMALL SPHERE}

A sphere of arbitrary size needs many $2^{\mathrm{n}}$-pole ccefficients $\left(a_{n}, b_{n}\right)$ to describe its scattered field components. When a sphere is electrically small, its dipole coefficients $\left(a_{1}, b_{1}\right)$ are the dominant terms in its scattered field expressions. We show in Fig. 60 the first three pairs of coefficients for a conducting sphere. As long as $p_{0}$ is less than 1.5, all coefficients increase monotonically with increasing $P_{\odot}$. One exception is that $b f\left(p_{0}\right)$ reaches its maximum strength of $-10.487+$ j 0.5) when $\rho_{0}$ is about 1.415. Numerically we list in Table IX some coefficients for three different sizes.

If we could satisfy ourselves by saying that all those coefficients smaller than $0.5 \%$ of the dominant coefficients $\left(a f, b_{1}^{c}\right)$ can be considered insignificant in the scattered field expressions in Eq. (13), then Table IX may be used to define three electrically small conducting spheres: A dipole sphere is that of $P_{0} \leq 0.2\left(d<0.064 \lambda_{o}\right)$ and has only one pair of significant coefficients $(a f, b q)$. A quadrupole sphere is that of $\rho_{0} \leq 1.0$ $\left(d<0.32 \lambda_{0}\right)$ and has two pairs of significant coefficients $\left(a q_{1}, b_{1}^{c}\right)$ and $\left(a \varepsilon_{2}, b_{2}^{c}\right)$. An octupole sphere is that of $p_{0} \leq i .5\left(d<0.48 \lambda_{0}\right)$ and has three pairs of significant coefficients $(a \mathcal{G}, b \mathcal{F}),\left(a_{2}^{c}, b_{2}^{c}\right)$ and $\left(a_{3}^{c}, b_{3}^{c}\right)$. 


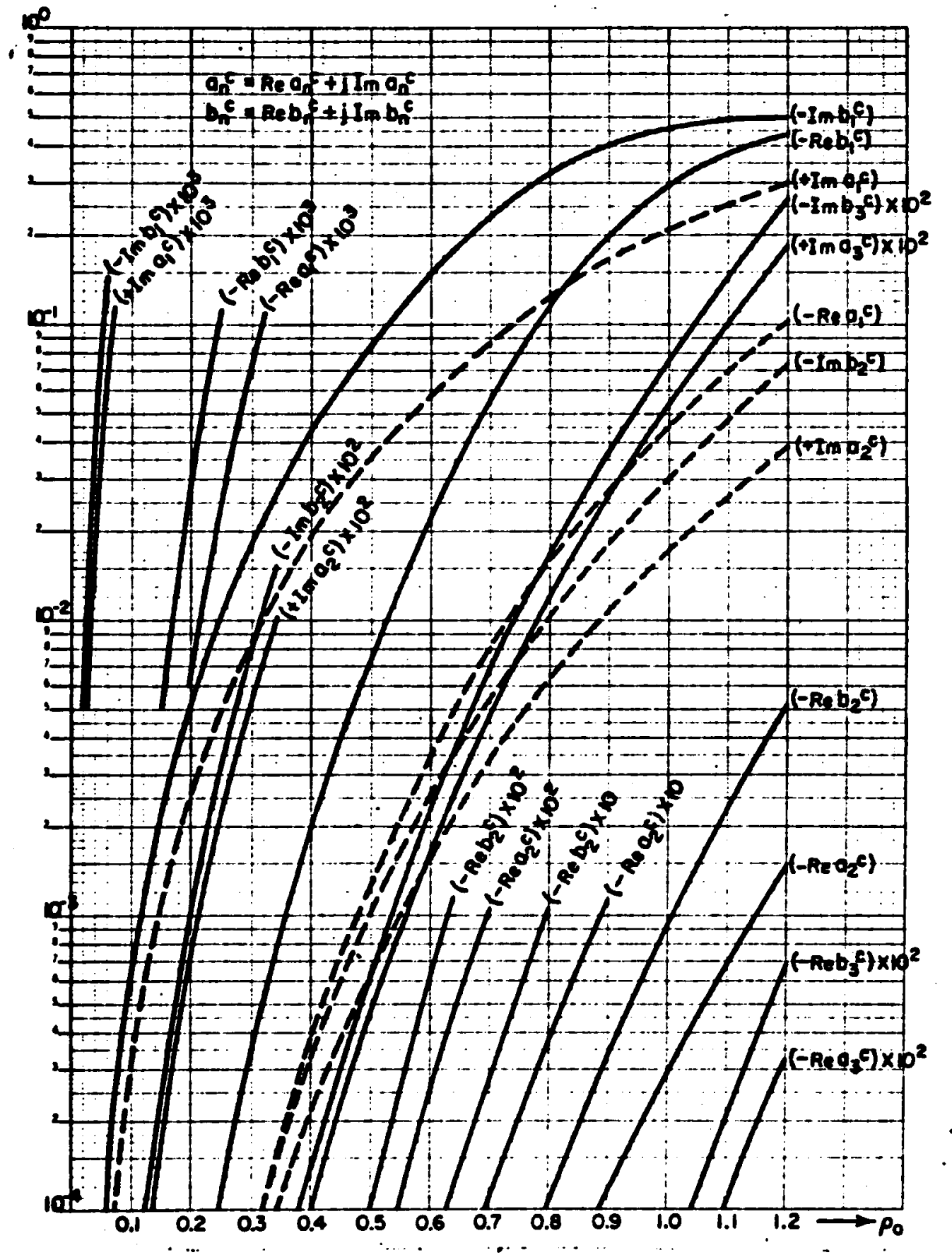

Fig. 60--The first three pairs of $2^{n}$-pole coefficients $\left(a_{n}^{c}, b_{n}^{c}\right)$ of a conducting sphere. 
TABLE IX

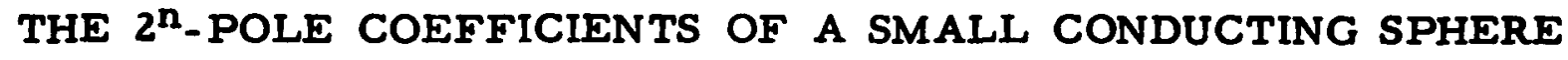

\begin{tabular}{|c|c|c|c|}
\cline { 2 - 4 } \multicolumn{1}{c|}{} & \multicolumn{2}{|c|}{$\rho_{0}=\pi d / \lambda_{o}\left(d\right.$ is diameter and $\lambda_{o}$ is free space wavelength) } \\
\cline { 2 - 5 } \multicolumn{1}{c|}{0.2} & 1.0 & 1.5 \\
\hline$a_{1}^{c}$ & $(-0.0069+j 2.6) \times 10^{-3}$ & $(-0.454+j 2.08) \times 10^{-1}$ & $(-2.445+j 4.298) \times 10^{-1}$ \\
$b_{1}^{c}$ & $(-0.029-j 5.4) \times 10^{-3}$ & $(-2.92-j 4.55) \times 10^{-1}$ & $(-4.801-j 4.996) \times 10^{-1}$ \\
\hline$a_{2}^{c}$ & $(-0.00005+j 7.04) \times 10^{-6}$ & $(-0.03+j 1.72) \times 10^{-2}$ & $(-0.888+j 9.38) \times 10^{-2}$ \\
$b_{2}^{c}$ & $(-0.00001-j 1.06) \times 10^{-5}$ & $(-0.09-j 3.04) \times 10^{-2}$ & $(-0.408-j 1.98) \times 10^{-1}$ \\
\hline$a_{3}^{c}$ & & $(-0.003+j 5.4) \times 10^{-4}$ & $(-0.056+j 7.48) \times 10^{-3}$ \\
$b_{3}^{c}$ & & $(-0.006-j 7.56) \times 10^{-4}$ & $(-0.013-j 1.13) \times 10^{-2}$ \\
\hline$a_{4}^{c}$ & & & $(-0.0009+j 2.96) \times 10^{-4}$ \\
$b_{4}^{c}$ & & & $(-0.0015-j 3.88) \times 10^{-4}$ \\
\hline
\end{tabular}


These three types of spheres are together called small spheres because their diameters are all smaller than one half of free space wavelength.

\section{A. Scattering Patterns of $A$ Conducting Sphere}

We use Eq. (13) with $n$ up to 4 to calculate the principal plane radar cross section $\sigma_{s}$ shown in Figs. 61 to 63 . If patterns for $P_{0}<0.8$ were plotted, one would find that they are similar to those for $p_{0}=0.8$, except that overall scattering cross sections become smaller, and the the E-plane minima become more pronounced. If patterns for $1.2<p_{0} \leq 1.5$ were plotted, one would find that the difference between $E$ - and $H$-plane become less and less, and that in some region of $\theta$ the $E$-plane cross sections become larger than those of H-plane. Specifically, we.plot the scattering cross sections at $\theta=0^{\circ}$ and $180^{\circ}$ as shown in Fig. 64 to indicate how they change with Po. One can see in this figure that a conducting sphere has a stronger backscattering as long as $p_{0}$ is smaller than 1.3 .

The H-plane phase patterns are seen in Fig. 63 as slowly varying function of $\theta$. On the other hand, the E-plane phase patterns have rapid change in $60^{\circ}<\theta<80^{\circ}$ of Fig. 62. This region of rapid phase change can be identified with the regions of minimum strength in the E-plane amplitude patterns in Figs. 61. From the standpoint of measurement accuracy, it is important to realize that a highly uncertain E-plane phase angle is expected in the neighborhood of the minimum strength 


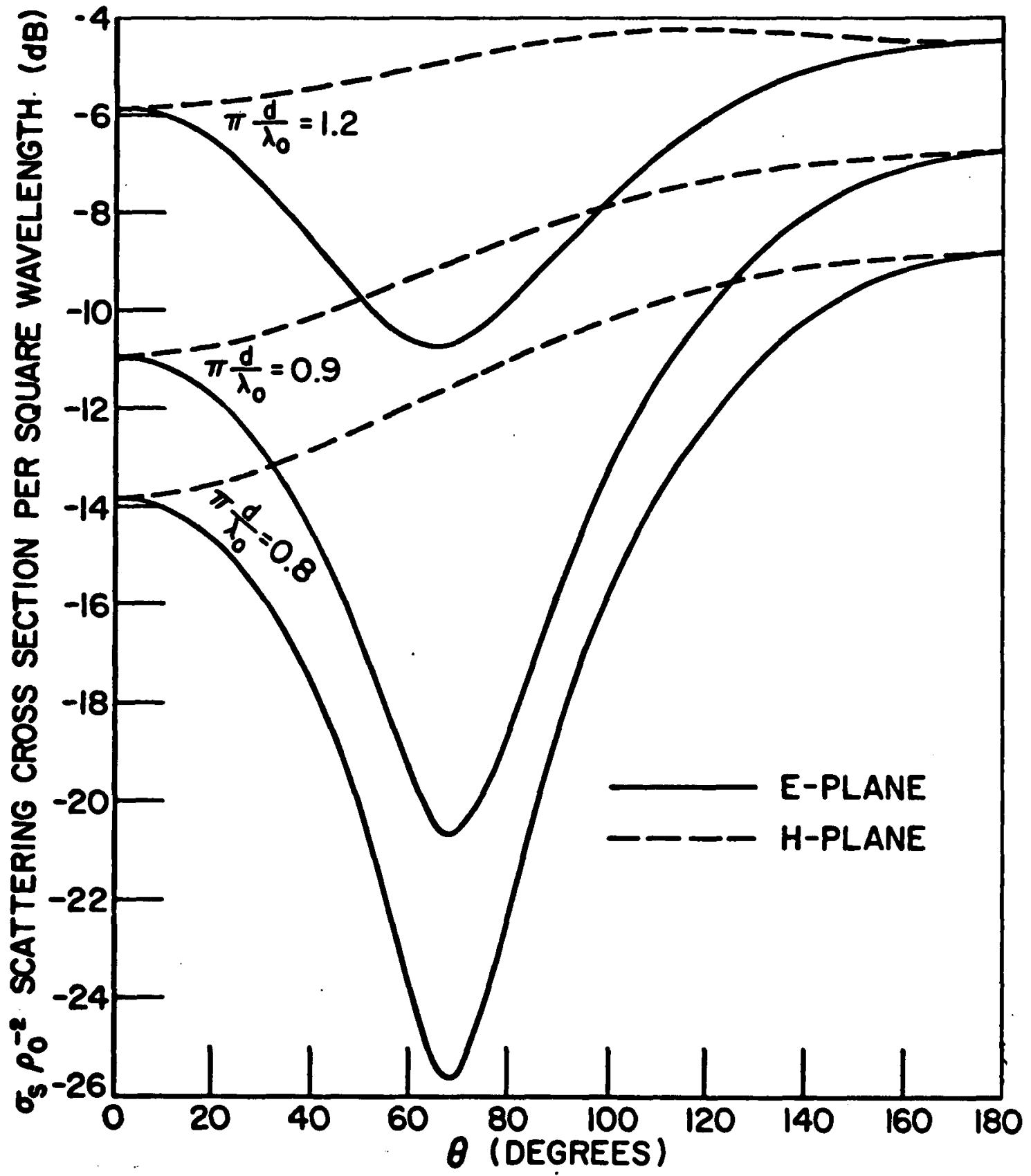

Fig. 61--The principal-plane amplitude patterns for three different sizes of a conducting sphere. 


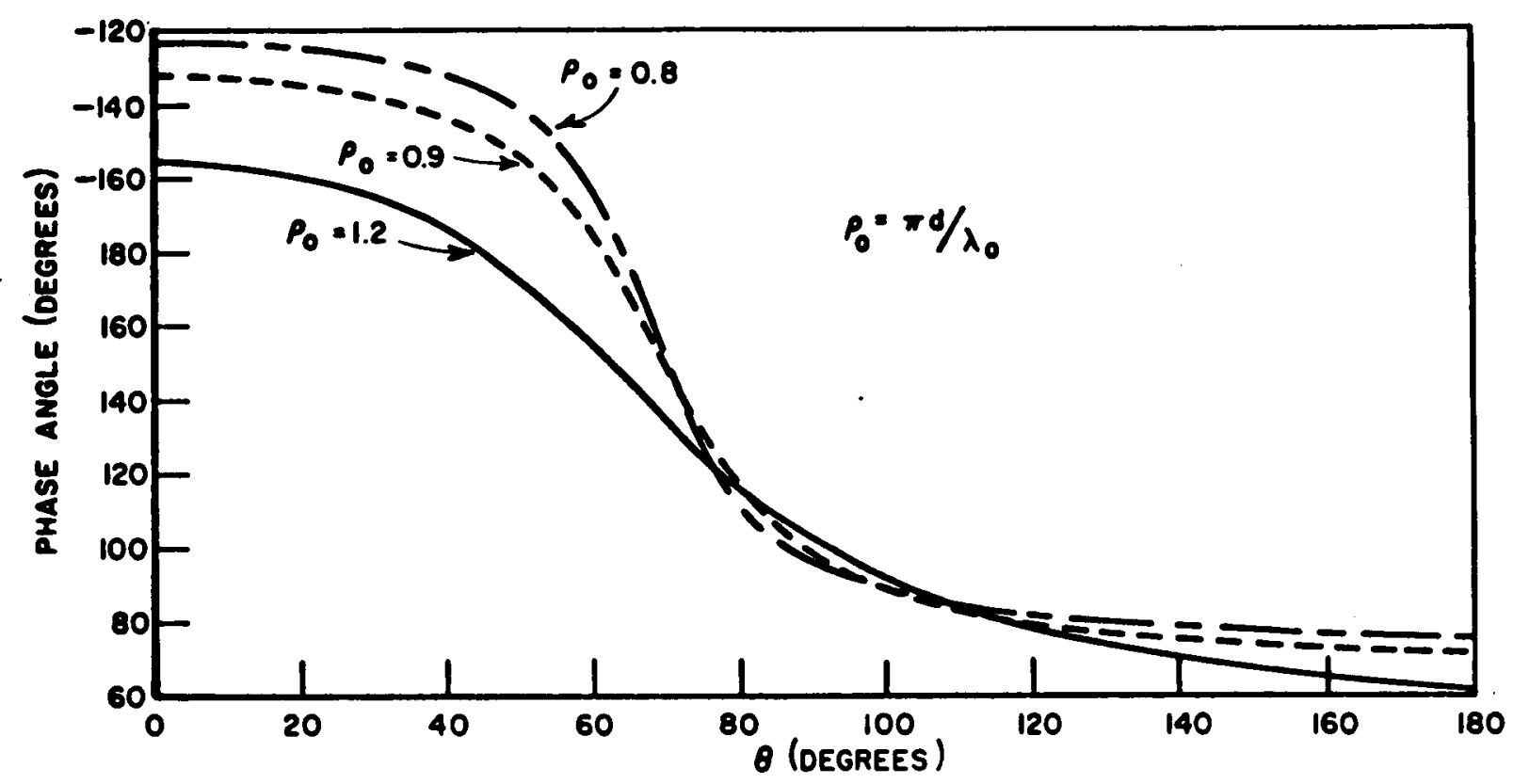

Fig. 62--The principal E-plane phase patterns for three different sizes of a conducting sphere.

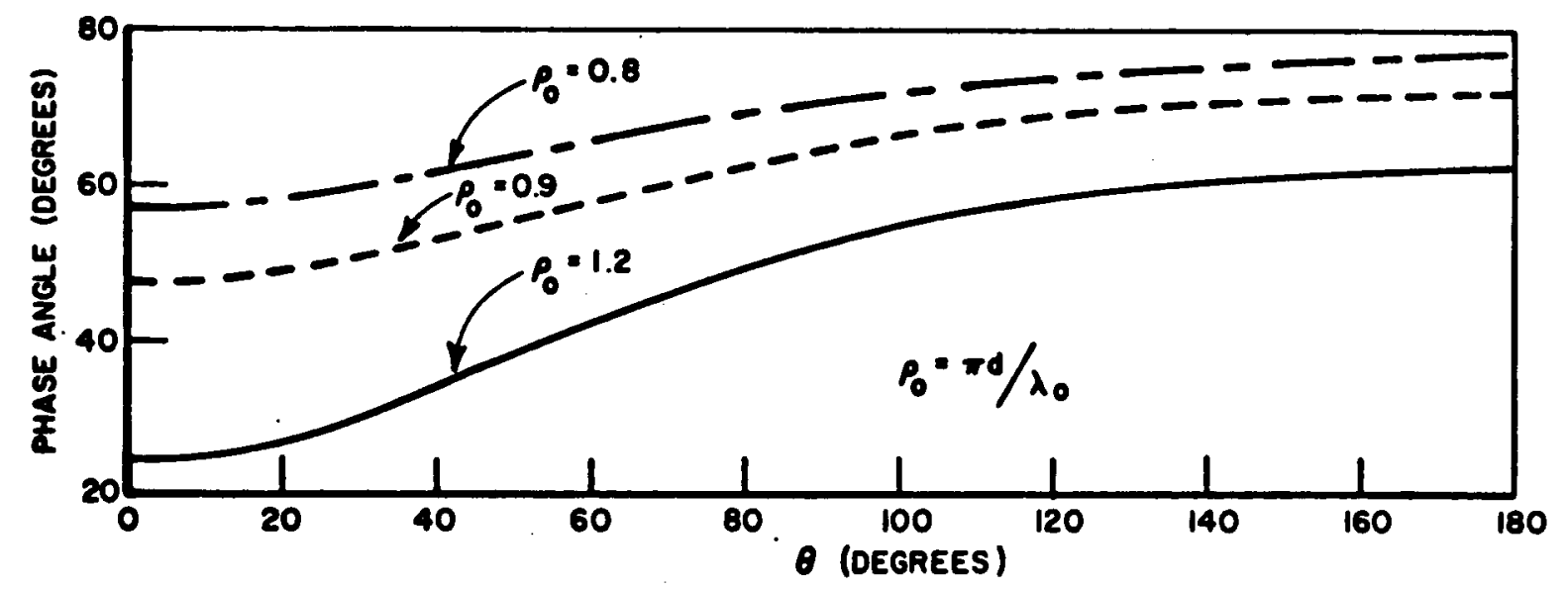

Fig. 63-- The principal H-plane phase patterns corresponding to those in Fig. 62 . 


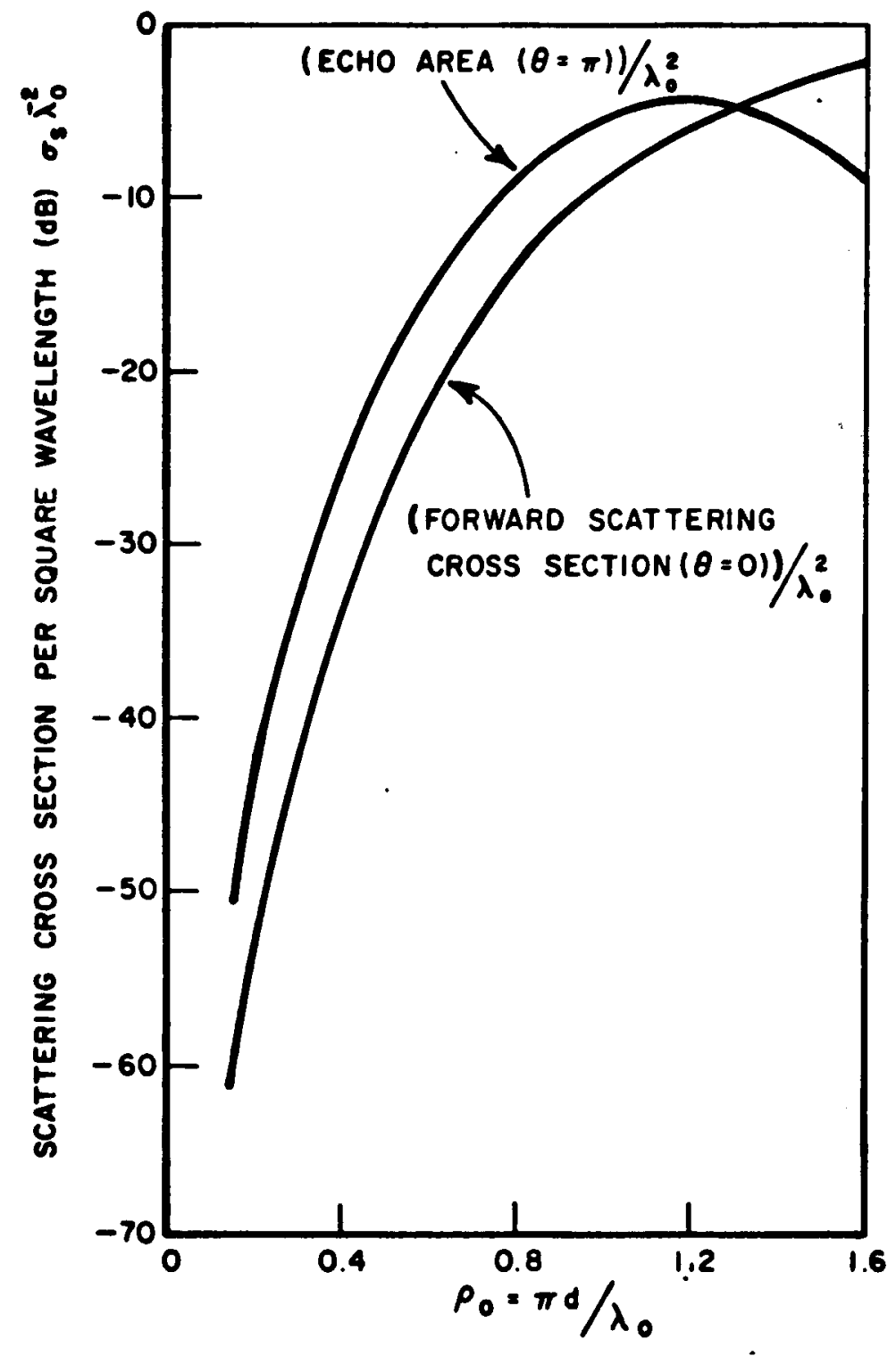

Fig. 64 --Forward and backscattering cross sections per square wavelength of a conducting sphere. 
in E-plane scattering. For instance, the E-plane phase angle of $p_{0}=0.8$ in Fig. 62 can change as much as $5^{\circ}$ for every change of $1^{\circ}$ in bistatic angle $\theta$.

B. Resonance Phenomena of

$2^{\mathrm{n}}$-pole Coefficients

We shall now proceed to discuss some resonance phenomena that could occur in a nonconducting sphere. The $2^{n}$-pole coefficients of a material sphere in general are defined in Eq. (6). We have described the function $X_{1}$ in Chapter III. Two other functions $A_{1}\left(\rho_{0}\right)$ and $B_{1}\left(\rho_{0}\right)$ used in the dominant coefficients $\left(a_{1}, b_{1}\right)$ are shown in Fig. 65. One can compare Eqs. $(7 a)$ and $(7 c)$ to see that $A_{1}$ and $X_{1}$ are identical functions if $v_{Y}$ is unity. The main purpose of studying resonance phenomena in $2^{n}$-pole coefficients is to assure ourselves that most typical specimen spheres can be classified as the three types classified for a small conducting sphere.

Let us first study $\left(a_{1}, b_{1}\right)$ as defined in Eq. (6) with $n=1$. In order to make them resonant, it is necessary to have $\eta_{r}^{-1} X_{1}$ and $\eta_{r} X_{1}$ about equal to $B_{1}$. Suppose a specimen sphere of $0.1 \lambda_{0}$ in diameter is to be made resonant. The value of $B_{1}(0.1 \pi)$ is about $-(2.85+j 0.09)$ in Fig. 65. In order to make $\left(a_{1}, b_{1}\right)$ resonant simultaneously, the value of $\eta_{x}$ needs to be nearly unity so that $\eta_{x}^{-1} X_{1}$ and $\eta_{x} x_{1}$ both would have the same value of $B_{1}(0.1 \pi)$. Let $\eta_{x}$ be $1.0-j 0.002$ as an illustration. Since the real part of $B_{1}(0.1 \pi)$ is -2.85 , it is now necessary to have an 


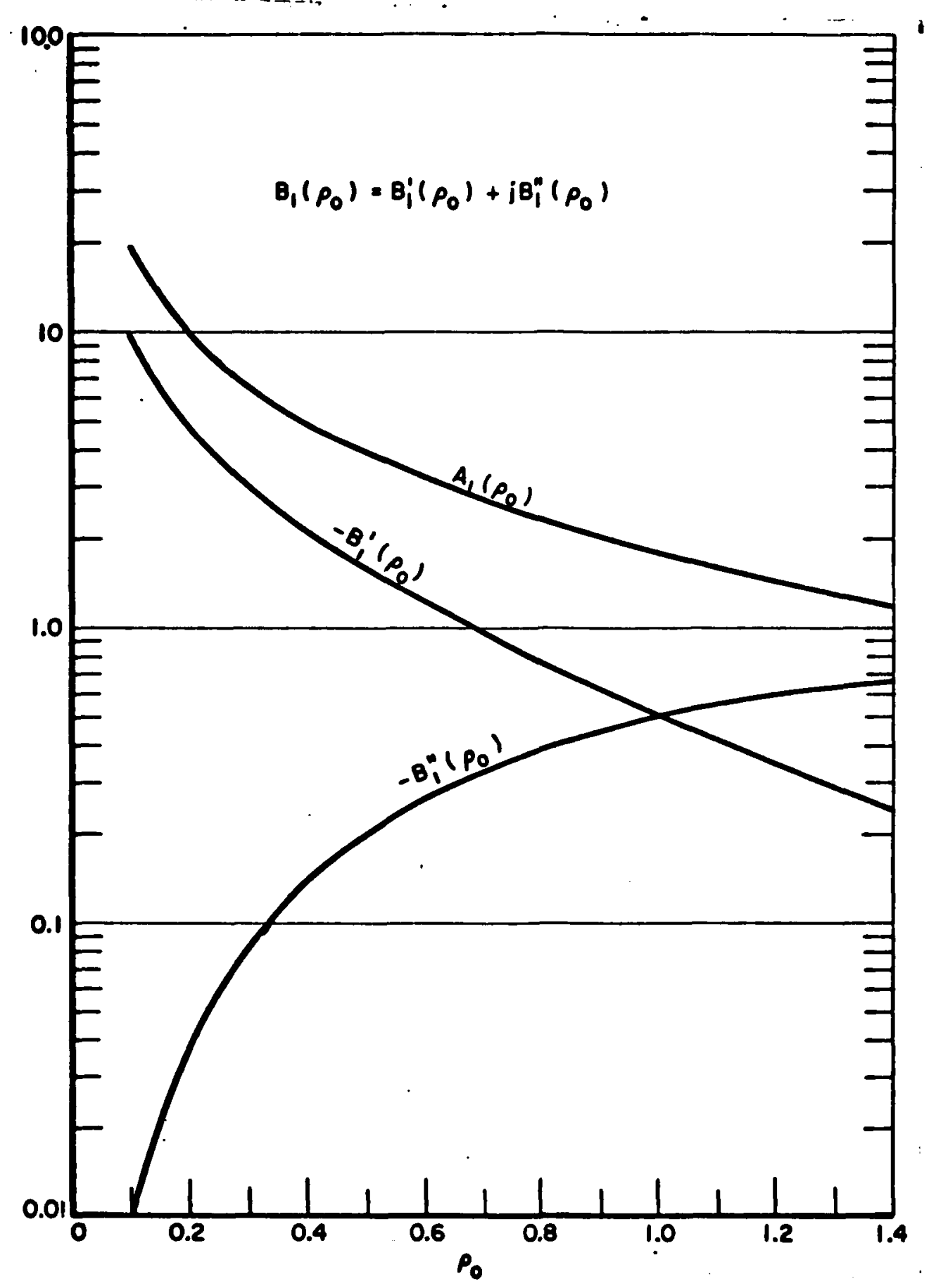

Fig. 65-- The functions $A_{1}\left(\rho_{0}\right)$ and $B_{1}\left(\rho_{0}\right)$ used to define the dipole coefficients $\left(a_{1}, b_{1}\right)$. 
$X_{1}$ whose real part is approximately equal to -2.85 . The smallest root for $X_{1}=-2.85+j 0$ can be found in Fig. 70 as $v_{r} P_{0}=4.15+j 0$. For a passive medium we choose $v_{r} \rho_{0}=4.15-\mathrm{j} 0.01$, and the resulting parameter $\left(\mu_{Y}, \epsilon_{Y}\right)$ are shown in Fig. 66. The forward scattering cross section per square wavelength in the figure is $-2.4 \mathrm{~dB}$. A conducting sphere of the same size $p_{0}=0.1 \pi$ is shown in Fig. 64 to have $-41 \mathrm{~dB}$. Therefore, one may consider that a gain of about $38 \mathrm{~dB}$ in forward scattering is achievable by making $a_{1}$ and $b_{1}$ resonant simultaneously. Another example in which only the magnetic dipole coefficient is made resonant is shown in Fig. 67. The sphere size is still $\rho_{0}=0.1 \pi$, but the parameters $\left(\eta_{r}, \rho_{o} v_{r}\right)=(0.35-j 0.05,3.695-j 0.058)$ are used. These two figures indicate that the conditions for dipole coefficients resonance can be satisfied only by those materials of extremely low losses but of large refractive indices. We note that our resonant strengths are referred to the corresponding coefficients of a conducting sphere of the same size. If a specimen sphere becomes larger, strengths of dipole resonance become weaker. This is because $\mathrm{X}_{1}$ shown in the refractive index chart always has a positive imaginary part which, together with $B_{1}\left(\rho_{0}\right)$ shown in Fig. 65, give an ever increasing value in the imaginary part of the denominators in $\left(a_{1}, b_{1}\right)$.

Similar analysis based on the recurrsion formulas in Eq. (7) indicate that any resonance in the higher-order $2^{n}$-pole coefficients requires a low loss material of high refractive index. The stronger 


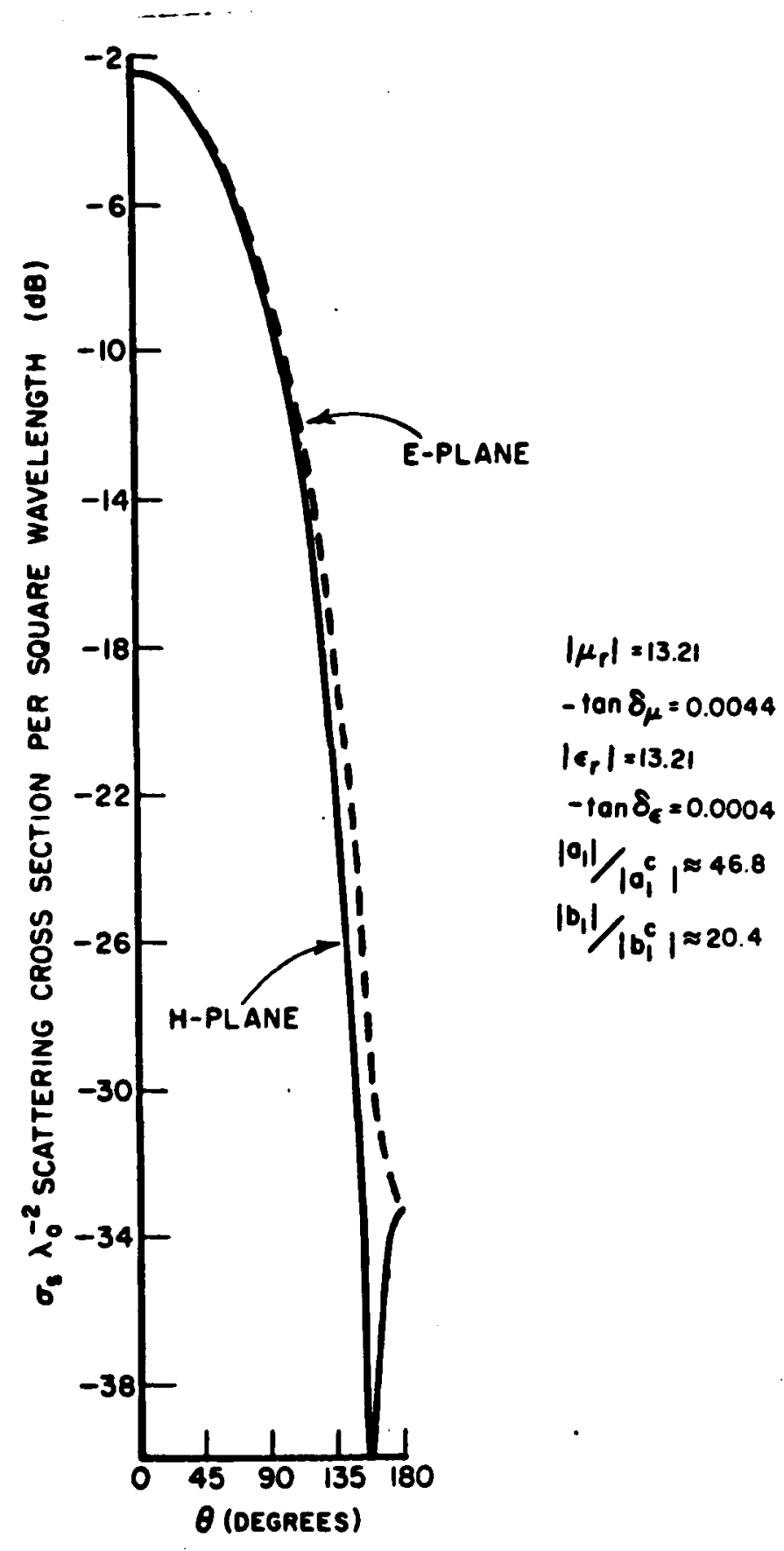

Fig. 66-- The principal-plane amplitude patterns of a sphere with $P_{0}=0.1 \pi$ when its dipole coefficients are simultaneously in resonance. 


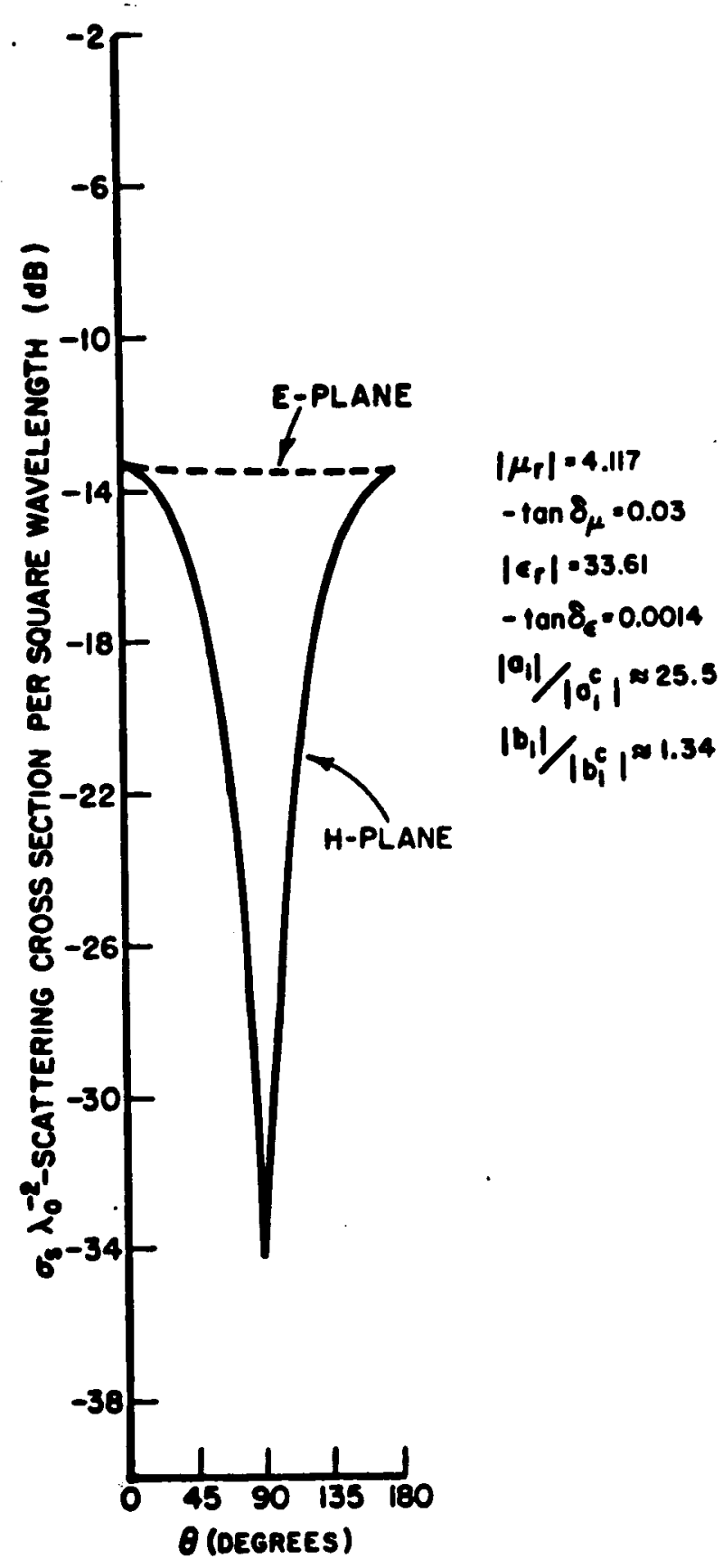

Fig. 67--The principal-plane amplitude patterns of

a sphere with $p_{o}=0.1 \pi$ when only its magnetic dipole is in resonance. 
the resonance strengths, the less lossier and the larger refractive index would be required. Therefore, for typical lossy materials the possibility of having strong resonances in $2^{n}$-pole coefficients $c a n$ be safely excluded from a specimen sphere. For examples, the $2^{n}$-pole coefficients of three different materials are shown in Table $\mathrm{X}$ where the data in the first column belong to a conducting sphere. The coefficients in the second column are all smaller than those of a conducting sphere,because the refractive index of this specimen is so small that its $X_{n}$ does not have a negative imaginary part. On the other hand, all the magnetic $2^{n}$-poles $a_{n}$ in the third column are slightly larger than the corresponding coefficients of the conducting sphere. This is because the products $\eta_{r}^{-1} X_{n}$ in $E_{q} \cdot(8 a)$ tend to make $a_{n}$ larger than $a_{n}^{c}$. However, the lossiness of this material prevents $a_{n}$ from being in strong resonance.

C. Dipoles, Quadrupole, and Octupole Spheres

So long as there is no higher-order $2^{\mathrm{n}}$-pole resonance, a specimen sphere of $p_{0} \leq 0.2$ is called a dipole sphere as was defined earlier for a conducting sphere. Similarly, a quadrupole and an octupole specimen sphere are called when their sizes are $p_{0} \leq \ldots 0$ and $\rho_{0} \leq 1.5$, respectively. It is in this respect that a specimen sphere and a conducting sphere of the same volume have somewhat similar characteristics in their scattering patterns. A quick glance on Figs. 45 
TABLE $X$

THE $2^{\text {n }}$-POLE COEFFICIENTS OF THREE SPHERES OF THE SAME SIZE BUT OF DIFFERENT MATERIALS

\begin{tabular}{|c|c|c|c|}
\cline { 2 - 5 } \multicolumn{1}{c|}{} & $\mu_{r} / \epsilon_{r} \rightarrow 0$ & $\begin{array}{l}\mu_{r} \mid=0.9984,-\tan \delta_{\mu}=0.00275 \\
\epsilon_{r} \mid=2.534,-\tan \delta_{\epsilon}=0.00639\end{array}$ & $\begin{array}{l}\left|\mu_{r}\right|=2.516,-\tan \delta_{\mu}=0.6598 \\
\epsilon_{r} \mid=19.01,-\tan \delta_{\epsilon}=0.0737\end{array}$ \\
\hline$a_{1}$ & $(-0.3328+j 1.794) \times 10^{-1}$ & $(-0.151-j 2.52) \times 10^{-2}$ & $(-1.807+j 1.307) \times 10^{-1}$ \\
\cline { 2 - 5 }$b_{1}$ & $(-2.321-j 4.222) \times 10^{-1}$ & $(-0.349-j 1.79) \times 10^{-1}$ & $(-2.474-j 2.117) \times 10^{-1}$ \\
\hline$a_{2}$ & $(-0.0163+j 1.275) \times 10^{-2}$ & $(-0.325-j 5.78) \times 10^{-4}$ & $(-1.963+j 0.357) \times 10^{-2}$ \\
\cline { 2 - 5 }$b_{2}$ & $(-0.0484-j 2.198) \times 10^{-2}$ & $(-0.135-j 8.52) \times 10^{-3}$ & $(-0.579-j 1.865) \times 10^{-2}$ \\
\hline$a_{3}$ & $(-0.0012+j 3.47) \times 10^{-4}$ & $(-0.68-j 7.8) \times 10^{-6}$ & $(-5.01-j 1.91) \times 10^{-4}$ \\
\cline { 2 - 5 }$b_{3}$ & $(-0.0023-j 4.81) \times 10^{-4}$ & $(-0.014-j 1.96) \times 10^{-4}$ & $(-0.747-j 4.443) \times 10^{-4}$ \\
\hline
\end{tabular}

$\rho_{0}=\pi d / \lambda_{0}=0.93557$ 
and 61 would reveal the similarity characteristic of a sphere with $p_{0}$ equal to 0.9 or 1.0 . However, there exists distinct differences between a specimen sphere and a conducting sphere. These differences are characteristics of different material parameters and these differences are used to determine the material parameters of a specimen sphere. 


\section{APPENDIX II \\ USEFUL APPLICATIONS AND DETAILED CONTOURS}

OF THE REFRACTIVE INDEX CHART

Some useful applications of the chart are to be pointed out here. Its principal application in finding complex roots for a value of $X_{1}$ has been illustrated in Chapters III and IV. The problem of determining a unique root $v_{\mathbf{r}} \rho_{0}$ from measured data has been studied in Chapter VI with the aid of the refractive index chart. The results shown in Fig. 45 are equivalent of the solutions for a synthesis problem of how to find three different materials which would exhibit essentially the same scattering characteristics. Another synthesis problem of finding a set of appropriate parameters to make dipole coefficients resonant has been illustrated by two examples in Figs. 66 and 67 .

The refractive index chart is also useful in calculating approximate $2^{n}$-pole coefficients defined in Eq. (6). For a small sphere, one can use Fig. 65 to obtain $A_{1}\left(p_{0}\right)$ and $B_{1}\left(p_{0}\right)$ and use the chart to obtain $X_{1}$ when $\rho_{0}$ and $v_{r}$ are given. By specifying the value of $\eta_{I}$, the values of $\left(a_{1}, b_{1}\right)$ can then be readily obtained as

$$
a_{1}=a_{1}^{c}\left(p_{0}\right) \frac{\eta_{I}^{-1} X_{1}\left(\nu_{I} p_{0}\right)-A_{1}\left(p_{0}\right)}{\eta_{I}^{-1} X_{1}\left(v_{I} p_{0}\right)-B_{1}\left(p_{0}\right)}
$$

and 


$$
b_{1}=a_{1}^{c}\left(\rho_{0}\right) \frac{\eta_{r} X_{1}\left(\nu_{Y} p_{0}\right)-A_{1}\left(\rho_{0}\right)}{\eta_{X} X_{1}\left(\nu_{Y} p_{0}\right)-B_{1}\left(\rho_{0}\right)},
$$

where $a_{1}^{c}\left(p_{0}\right)$ can be found by Fig. 60. By use of the values $A_{1}\left(p_{0}\right)$, $B_{1}\left(\rho_{0}\right)$ and $X_{1}\left(\nu_{r} \rho_{0}\right)$ their corresponding higher-order quantities can be obtained by the recurrence formulas in Eq. (9). Subsequently, with the aid of Fig. 60, all the $2^{\mathrm{n}}$-pole coefficients in Eq. (6) can be obtained in the same manner as that for $\left(a_{1}, b_{1}\right)$. This application is, of course, based on the assumption that no computer program is available for calculating spherical Bessel functions of complex arguments .

Accuracy of using the refractive index chart depends on region of interest. In general, for a specified value of $v_{r} \rho_{0}, X_{1}$ can be obtained by use of the chart to much less than $5 \%$-uncertainty. As an extreme example, we use $1.05 \mathrm{X}_{1}$ in Eq. (7c) to generate all the subsequent $X_{n}$ for $n=2,3$ and 4 . The values of these approximate $X_{n}$ are then substituted into $\mathrm{Eq} \cdot(6)$ to calculate the $2^{\mathrm{n}}$-pole coefficients. These coefficients are then in turn used to compute scattering patterns. Final results are shown in Figs. 68 and 69 to indicate that close agreement with the true patterns can be obtained. Generally much better agreements could be expected if $X_{1}$ were more carefully and accurately obtained by the chart.

Finally we show in Figs. 70 to 85 the detailed contours of $X_{1}$, as we have already outlined the chart in Fig. 9. 


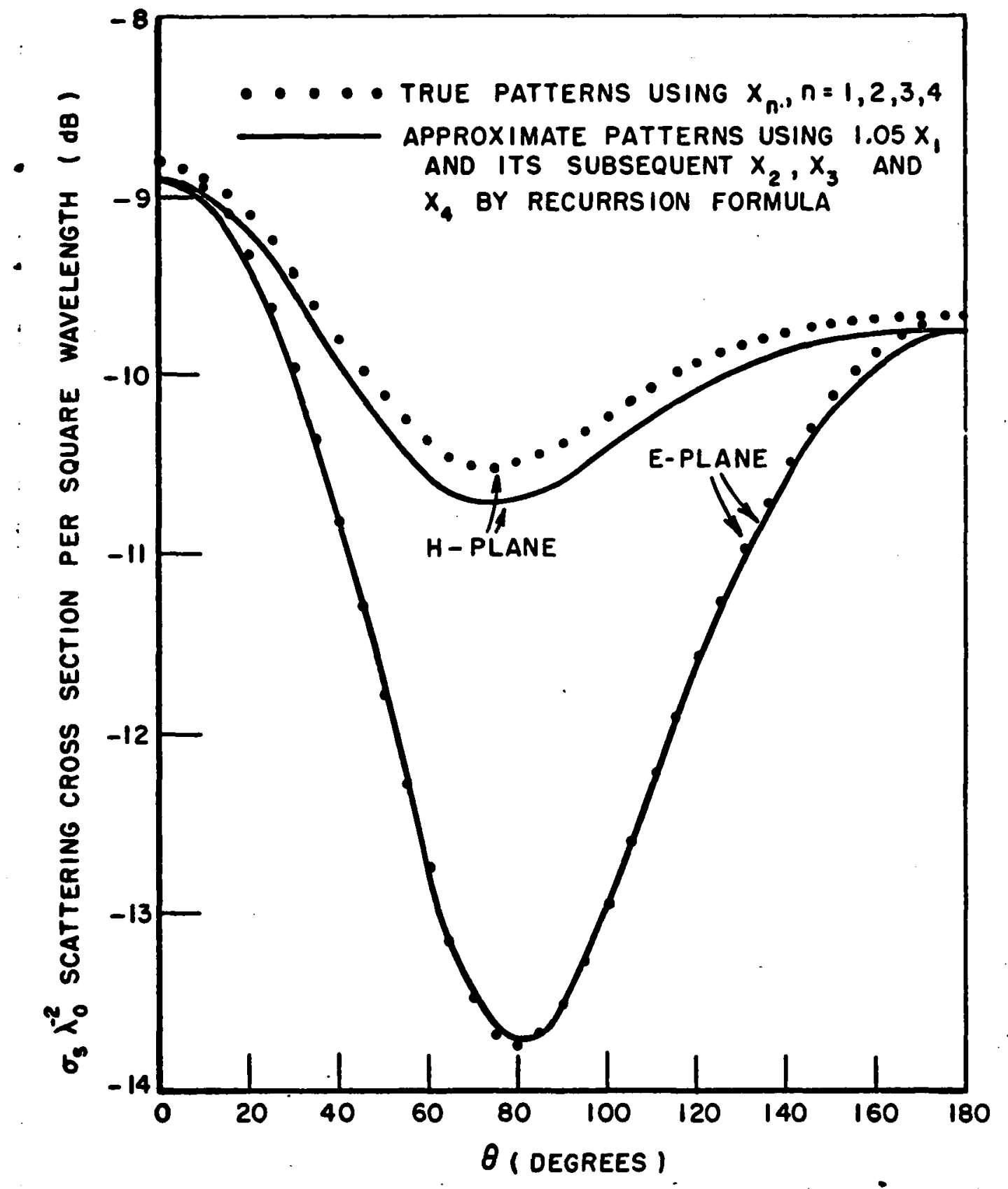

Fig. 68--True amplitude patterns compared with those obtained by using an approximate $X_{1}$. 


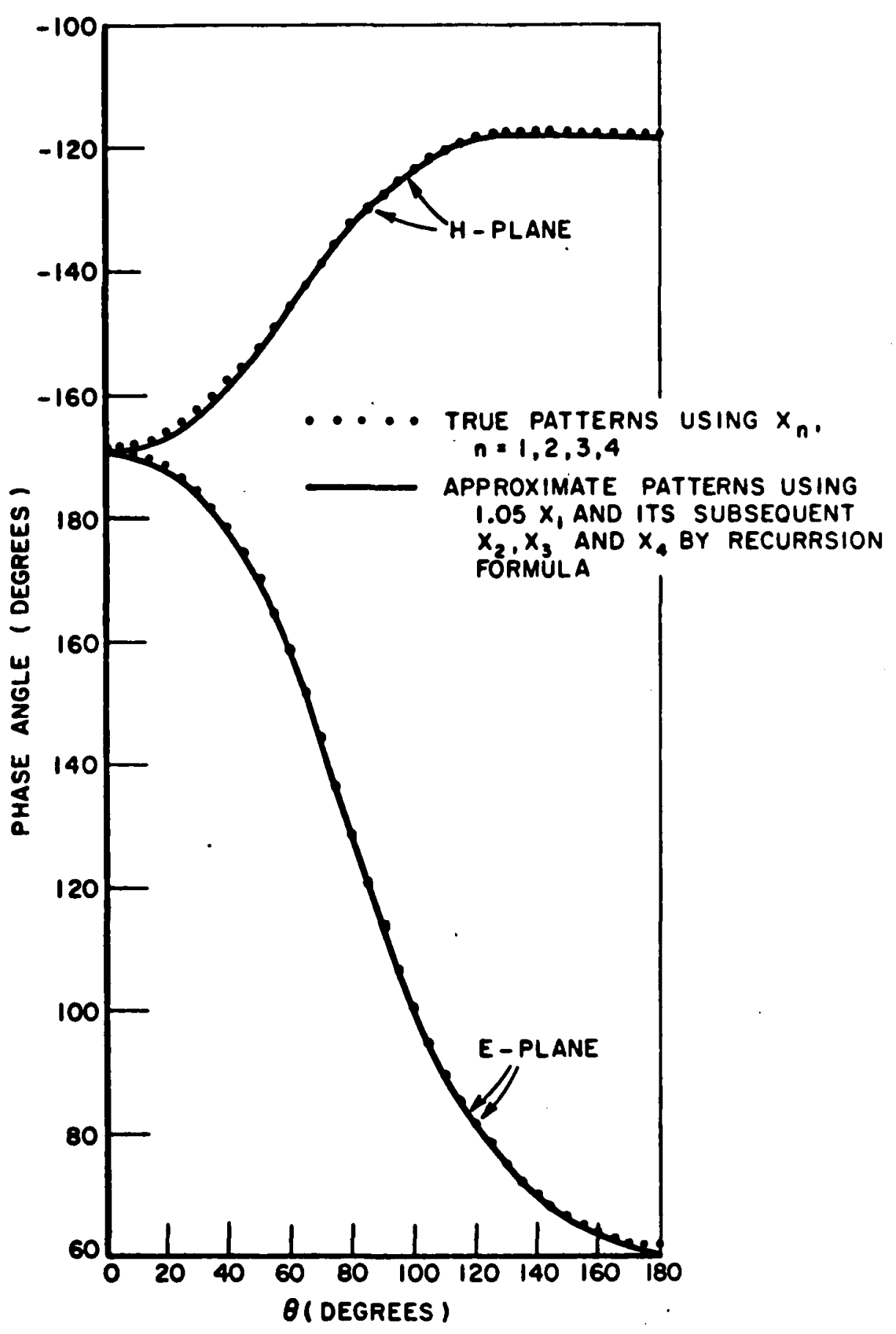

Fig. 69--True phase patterns compared with those obtained by using an approximate $X_{1}$. 


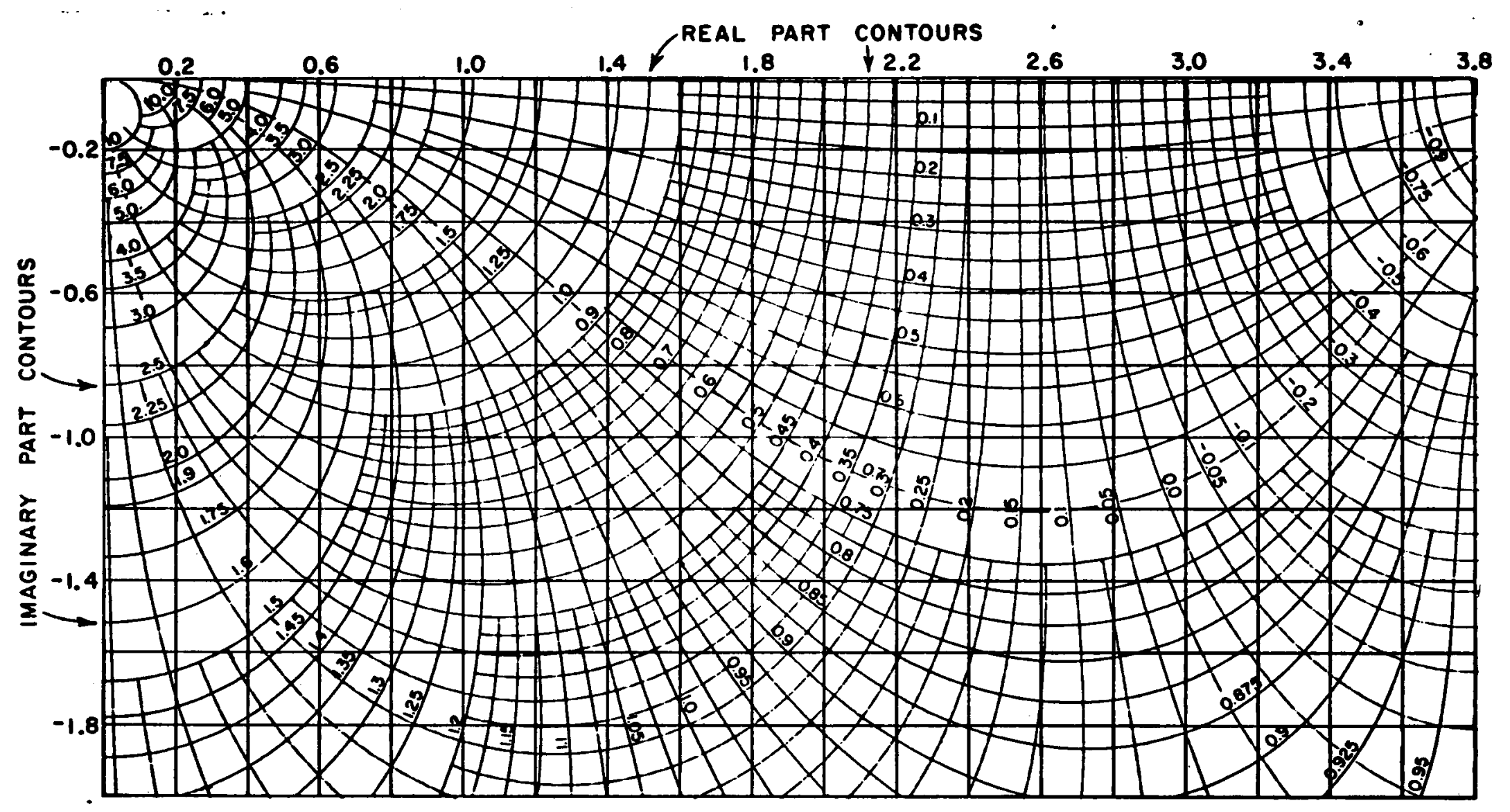

Fig. 70--Contours of $X_{1}\left(v_{r} \rho_{0}\right)$ in the region $0 \leq v_{r}^{\prime} \rho_{0} \leq 3.8$ and $0 \geq v_{r} " \rho_{0} \geq-2.0$. 


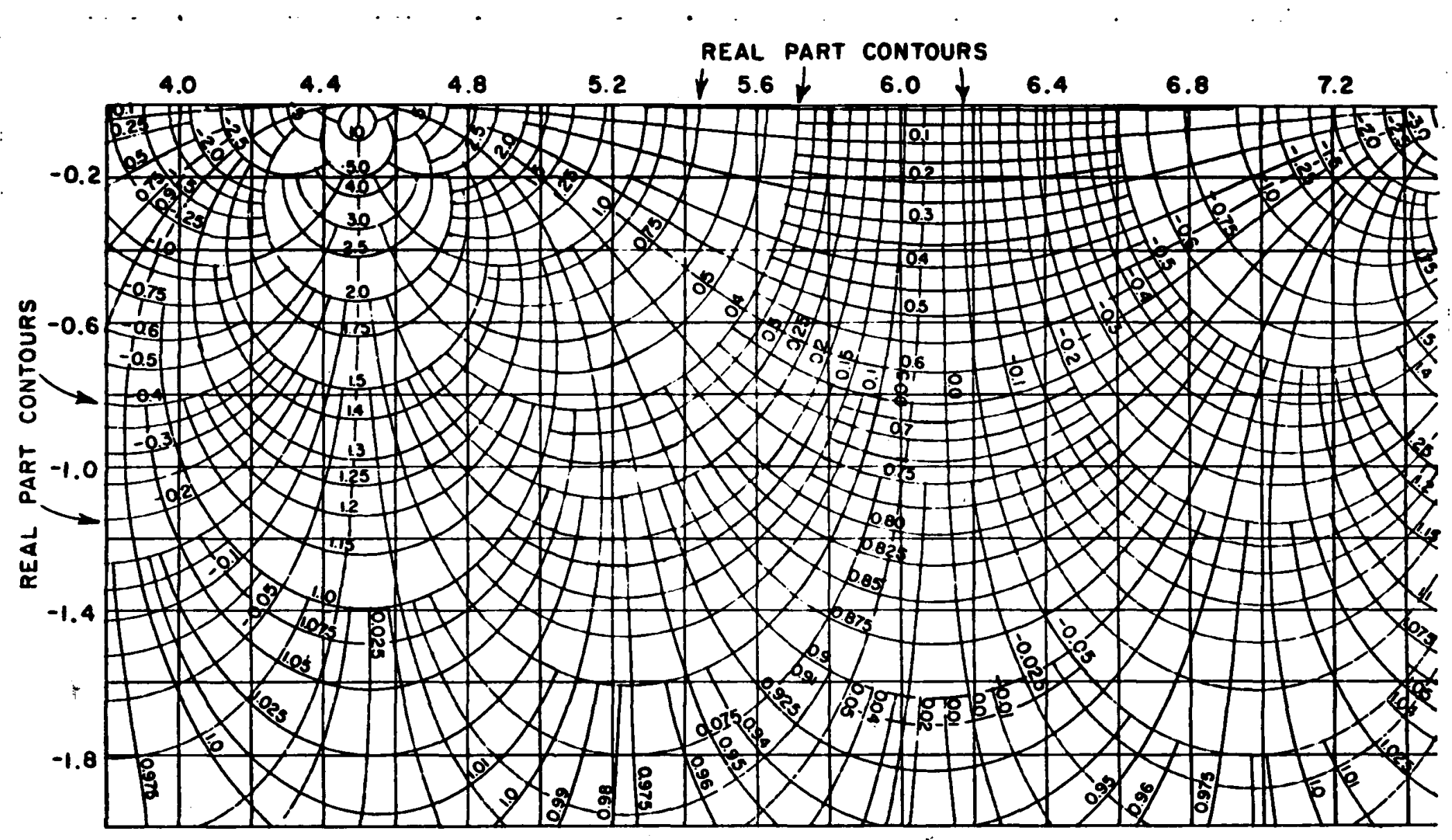

Fig. 71 --Contours of $X_{1}\left(v_{r} \rho_{O}\right)$ in the region $3.8 \leq v_{r} \rho_{0} \leq 7.5$ and $0 \geq v_{r} " \rho_{0} \geq-2.0$. 



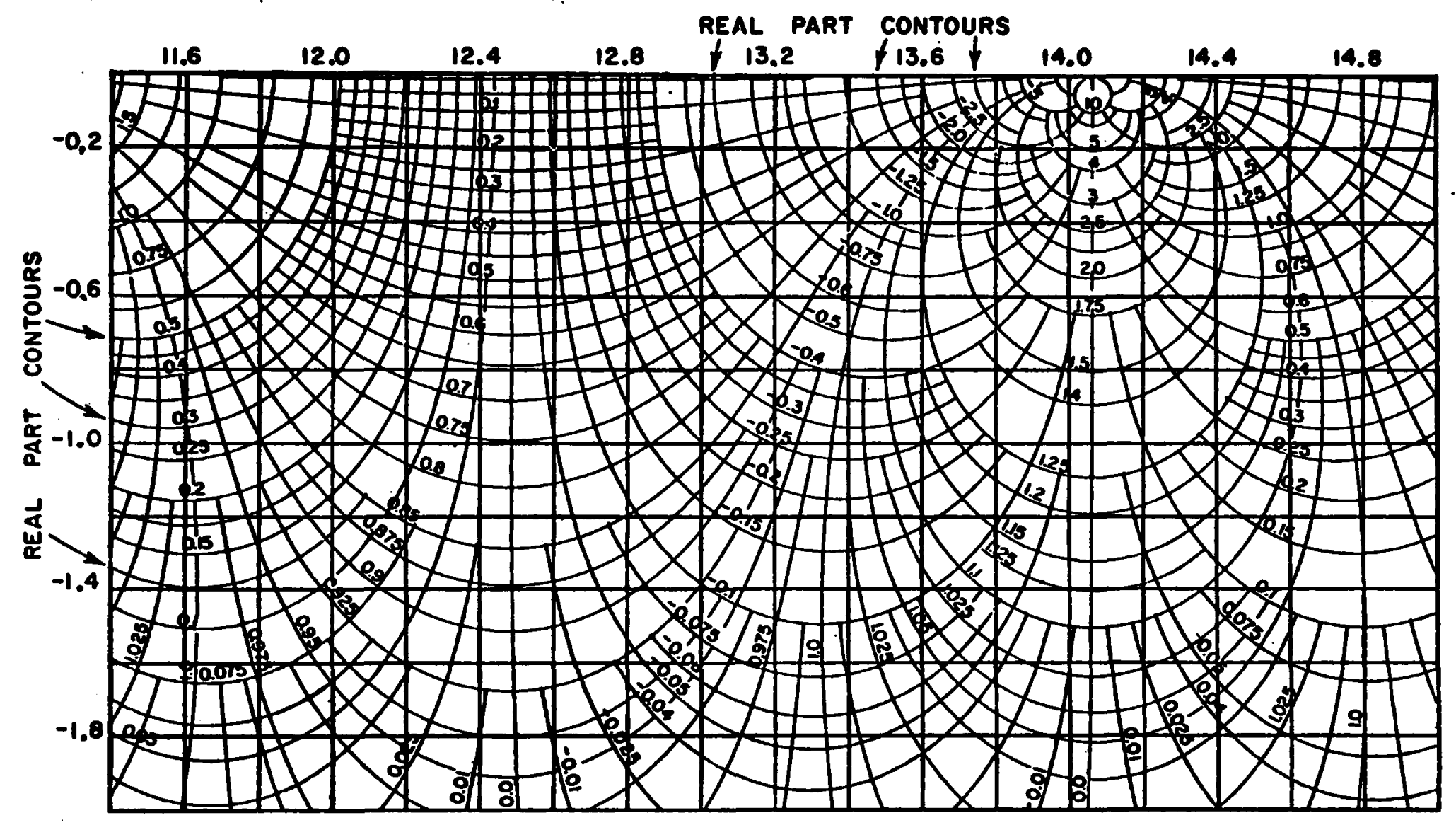

Fig. 73--Contours of $X_{1}\left(v_{r} \rho_{0}\right)$ in the region $11.4 \leq v_{r} \rho_{0} \leq 15.0$ and $0 \geq v_{r} " \rho_{0} \geq-2.0$. 


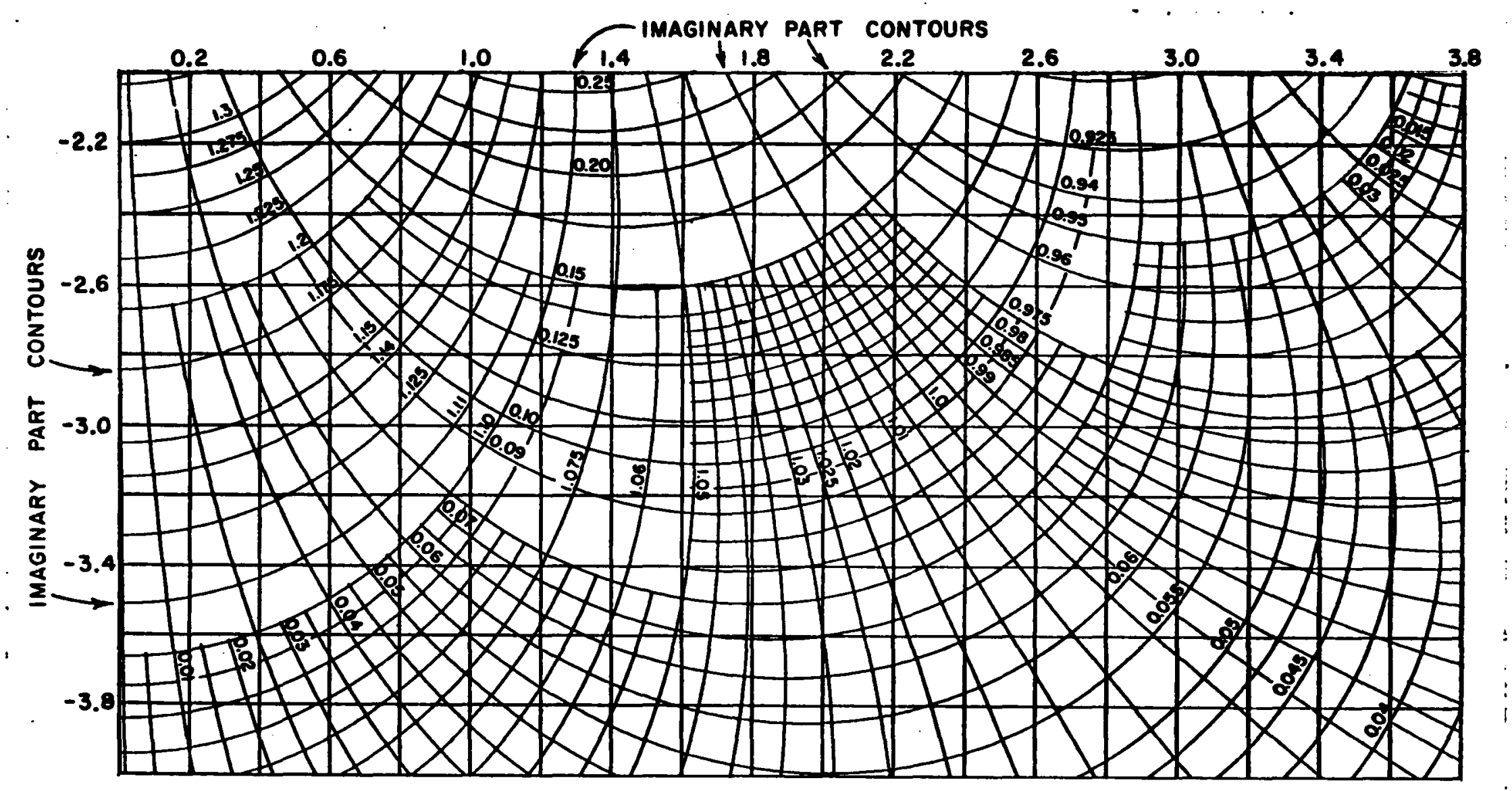

Fig. 74--Contours of $X_{1}\left(v_{r} \rho_{0}\right)$ in the region $0 \leq \nu_{r} \rho_{0} \leq 3.8$ and $-2.0 \geq v_{r} " \rho_{0} \geq-4.0$. 



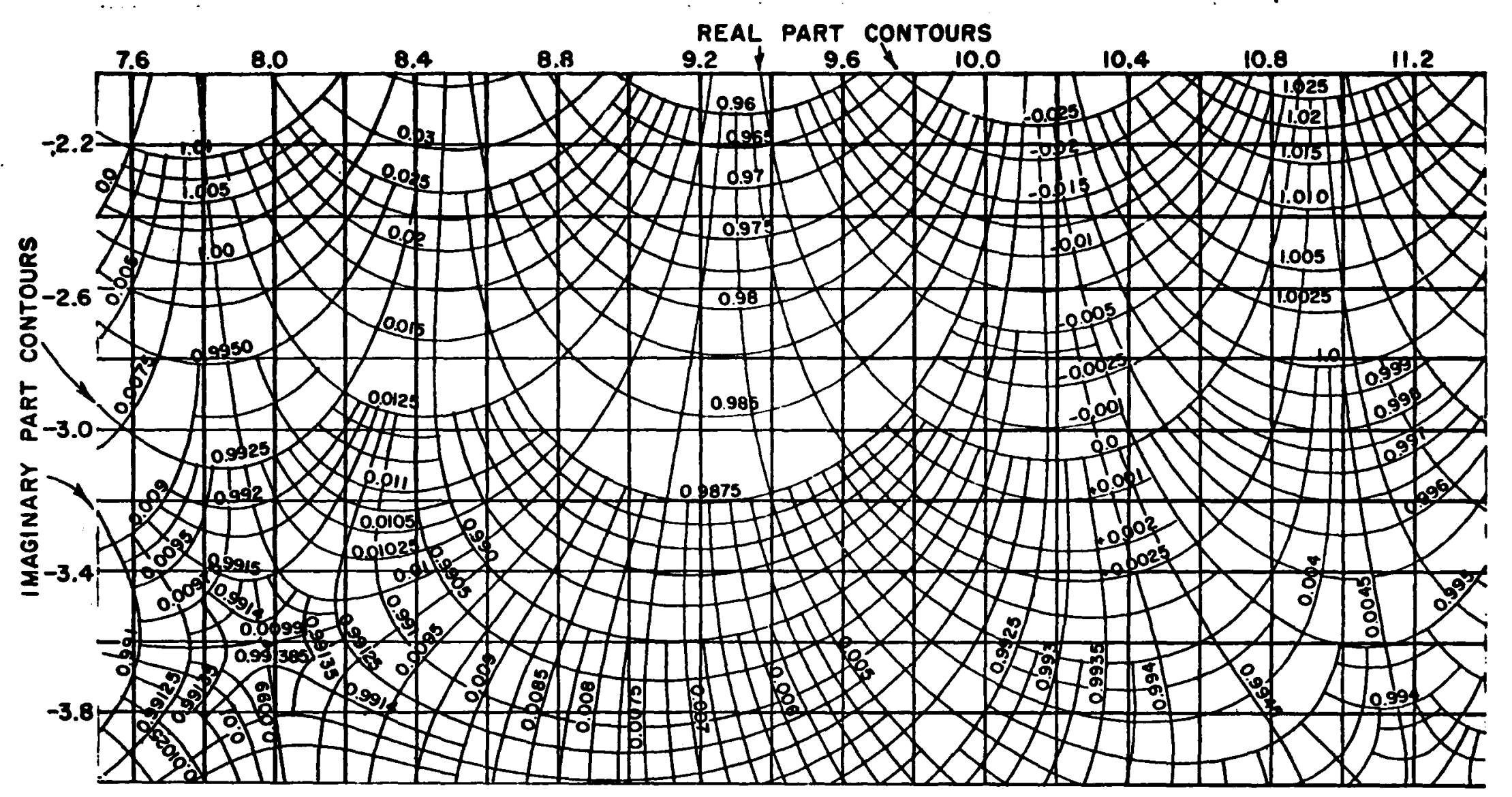

Fig. 76--Contours of $X_{1}\left(v_{r} \rho_{O}\right)$ in the region $7.5 \leq v_{r} \rho_{0} \leq 11.4$ and $-2.0 \geq v_{r} " \rho_{0} \geq-4.0$. 


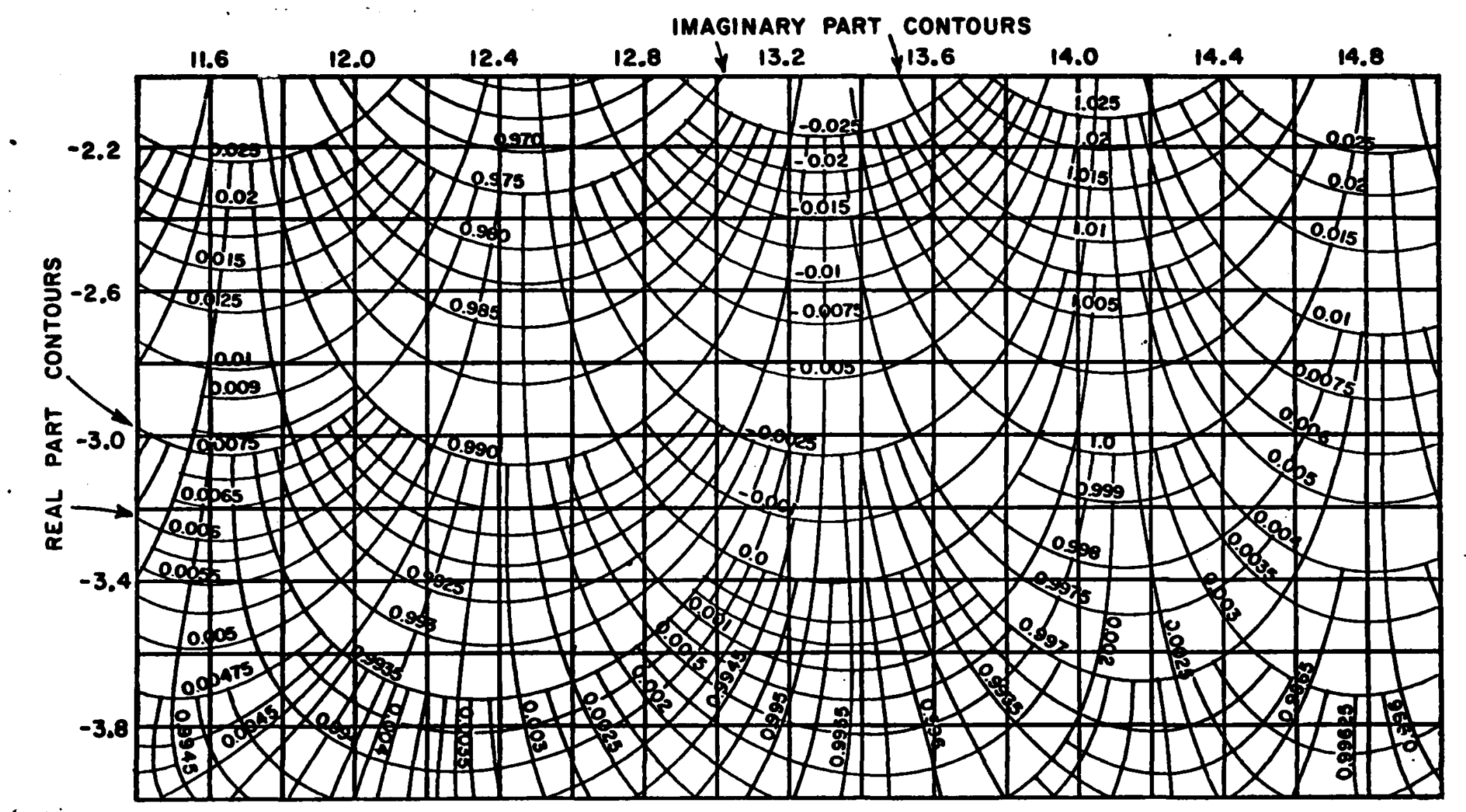

Fig. 77--Contours of $X_{1}\left(v_{r} \rho_{0}\right)$ in regions $11.4 \leq v_{r} \rho_{0} \leq 15.0$ and $-2.0 \geq v_{r} " \rho_{0} \geq-4.0$. 




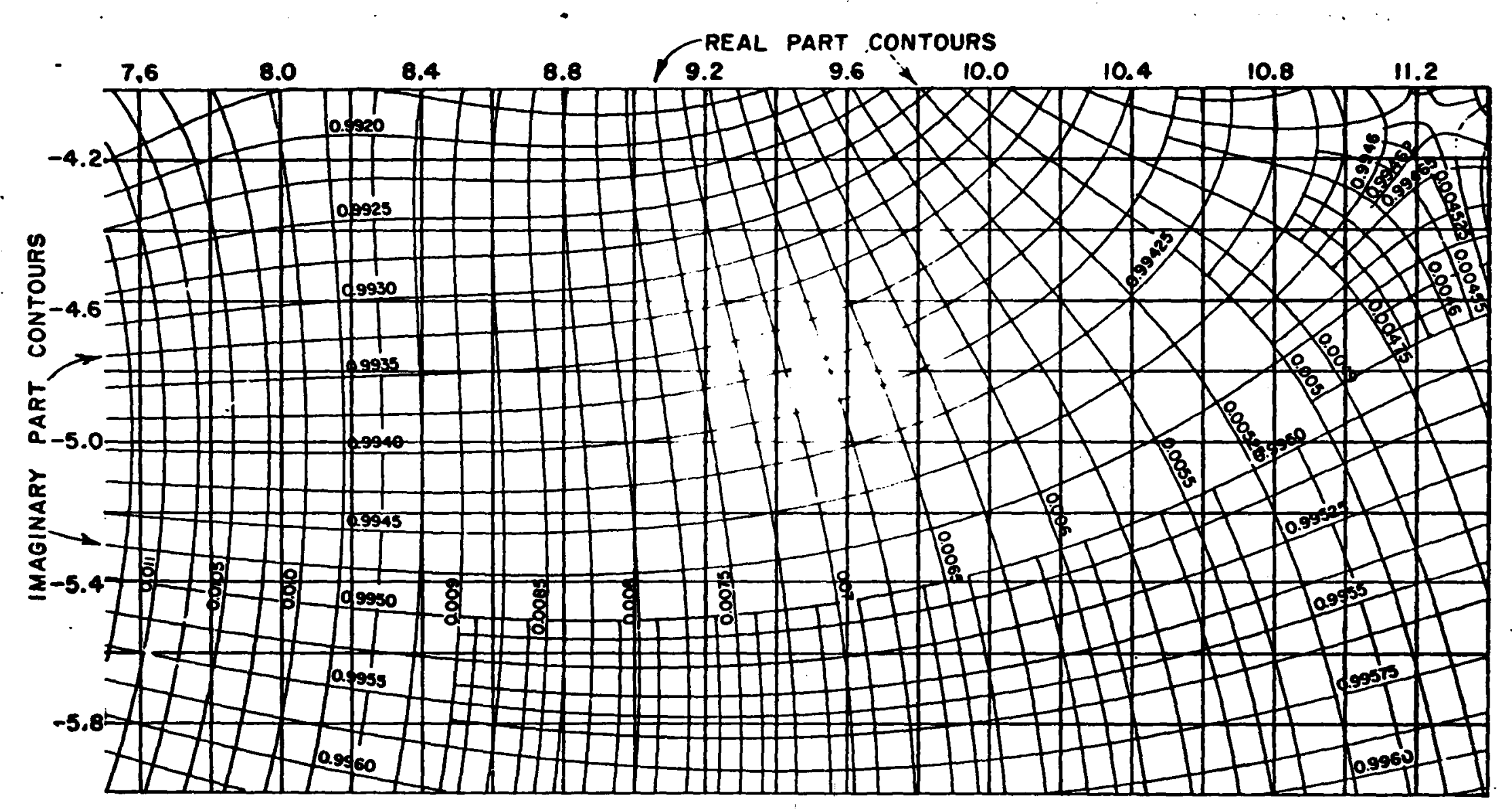

Fig. 80--Contours of $X_{1}\left(v_{r} \rho_{O}\right)$ in the region $7.5 \leq v_{r}^{\prime} \rho_{o} \leq 11.4$ and $-4.0 \geq v_{r} " \rho_{o} \geq-6.0$. 


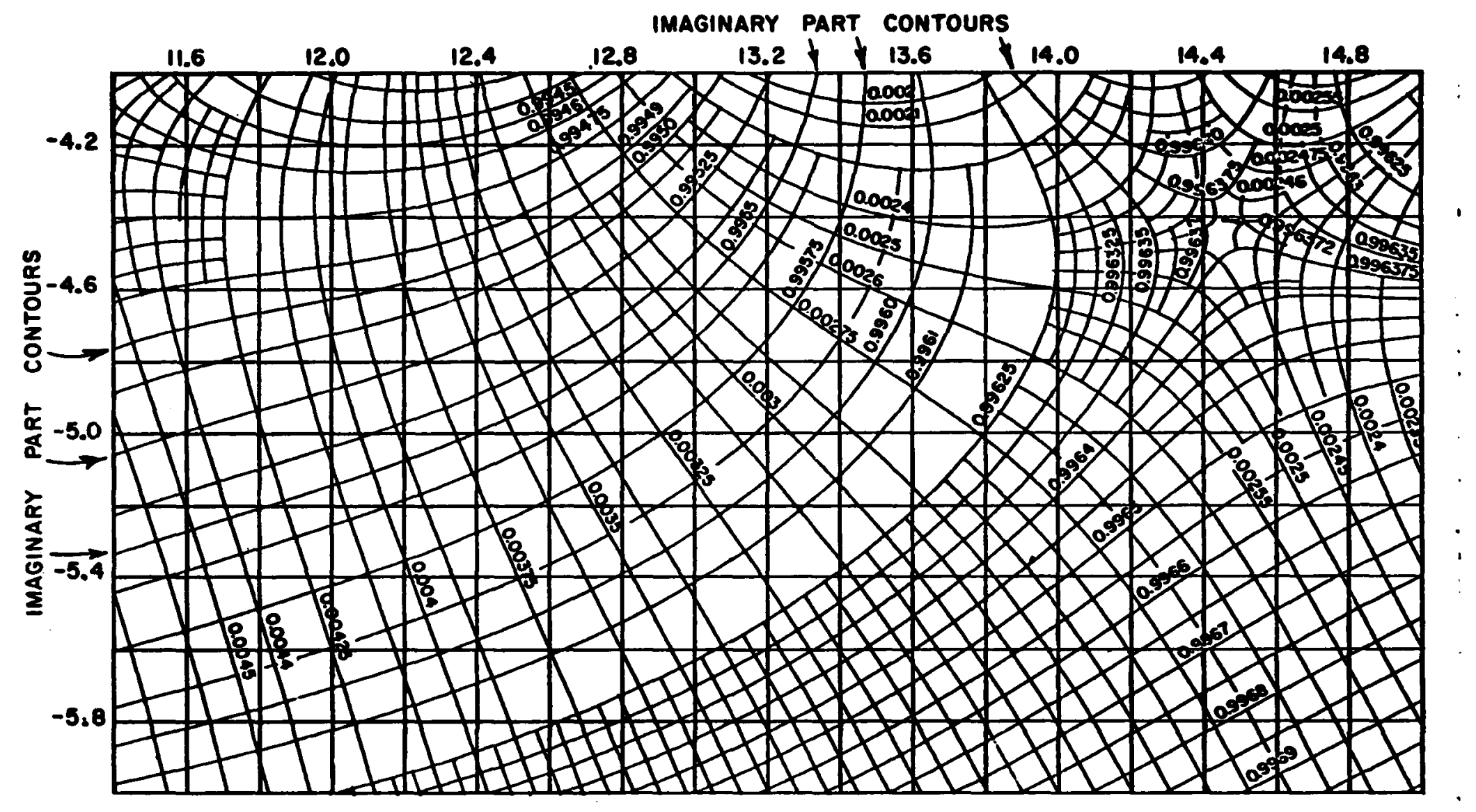

Fig. 81--Contours of $x_{1}\left(v_{r} p_{0}\right)$ in the region $11.4 \leq v_{r}^{\prime} p_{0} \leq 15.0$ and $-4.0 \geq v_{r} " p_{0} \geq-6.0$. 


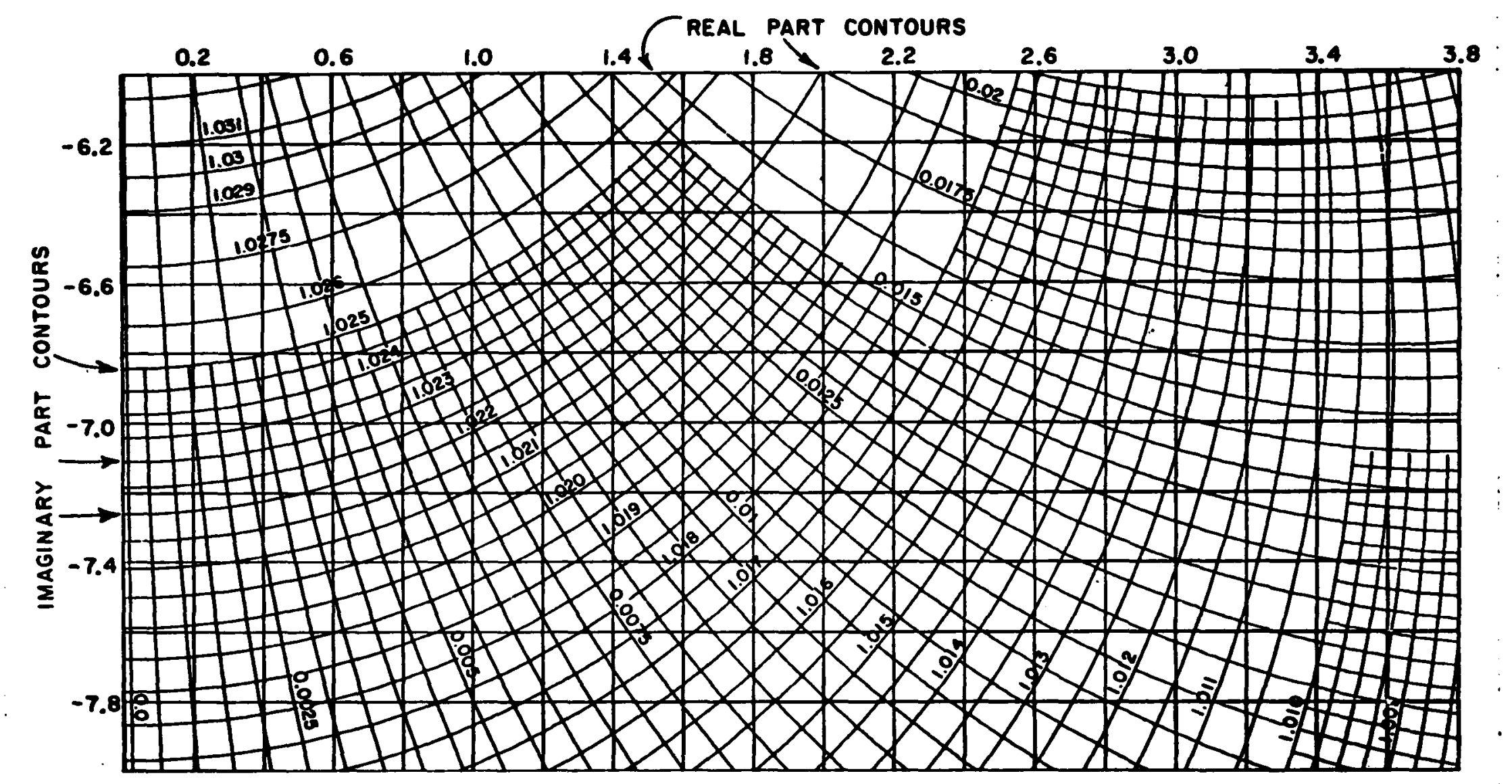

Fig. 82--Contours of $X_{1}\left(v_{r} \rho_{0}\right)$ in the region $0 \leq v_{r} \rho_{0} \leq 3.8$ and $-6.0 \geq v_{r} " \rho_{0} \geq-8.0$. 


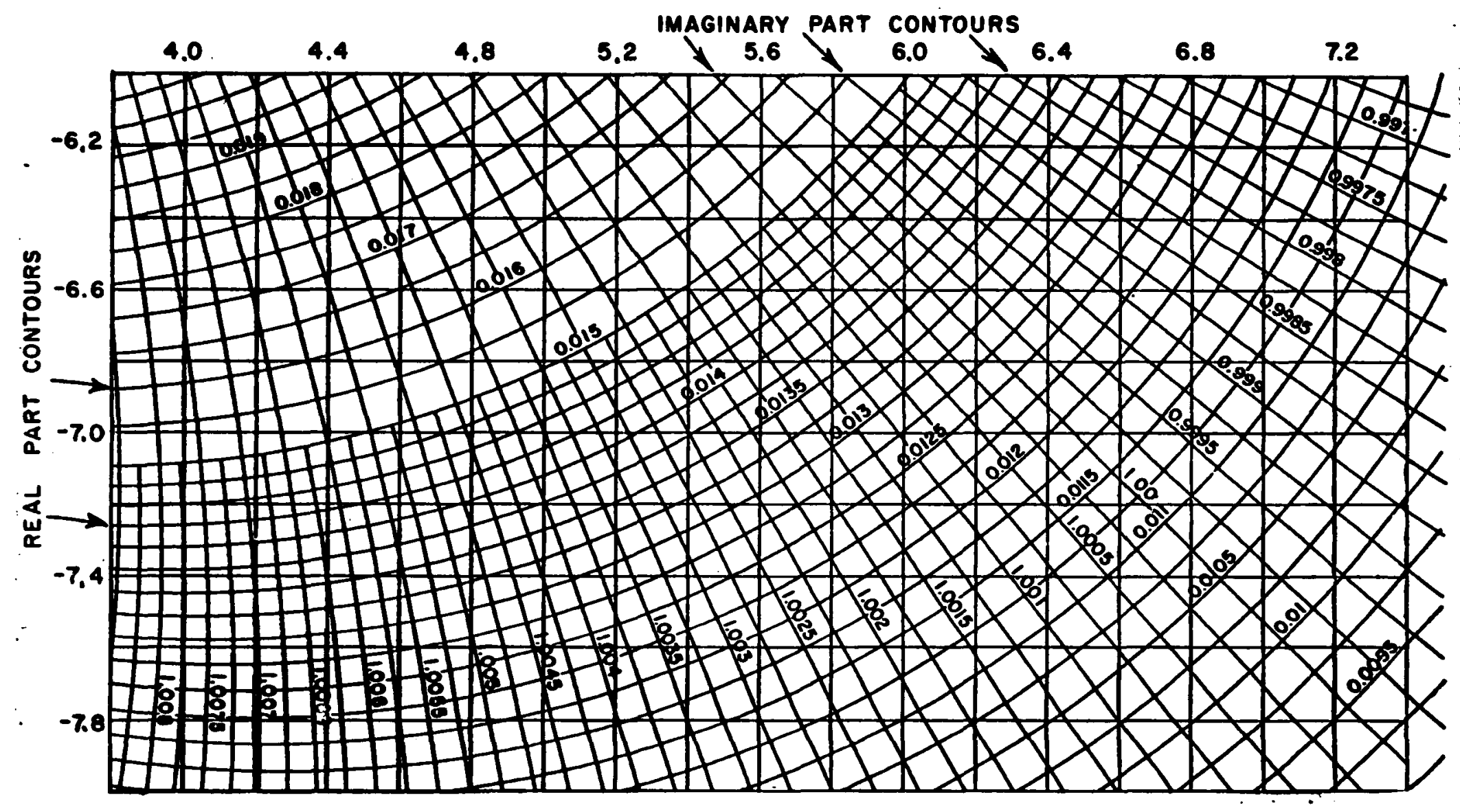

Fig. 83--Contours of $X_{1}\left(v_{r} \rho_{O}\right)$ in the region $3.8 \leq \nu_{r}^{\prime} \rho_{O} \leq 7.5$ and $-6.0 \geq v_{r}{ }^{\prime \prime} \rho_{o} \geq-8.0$. 



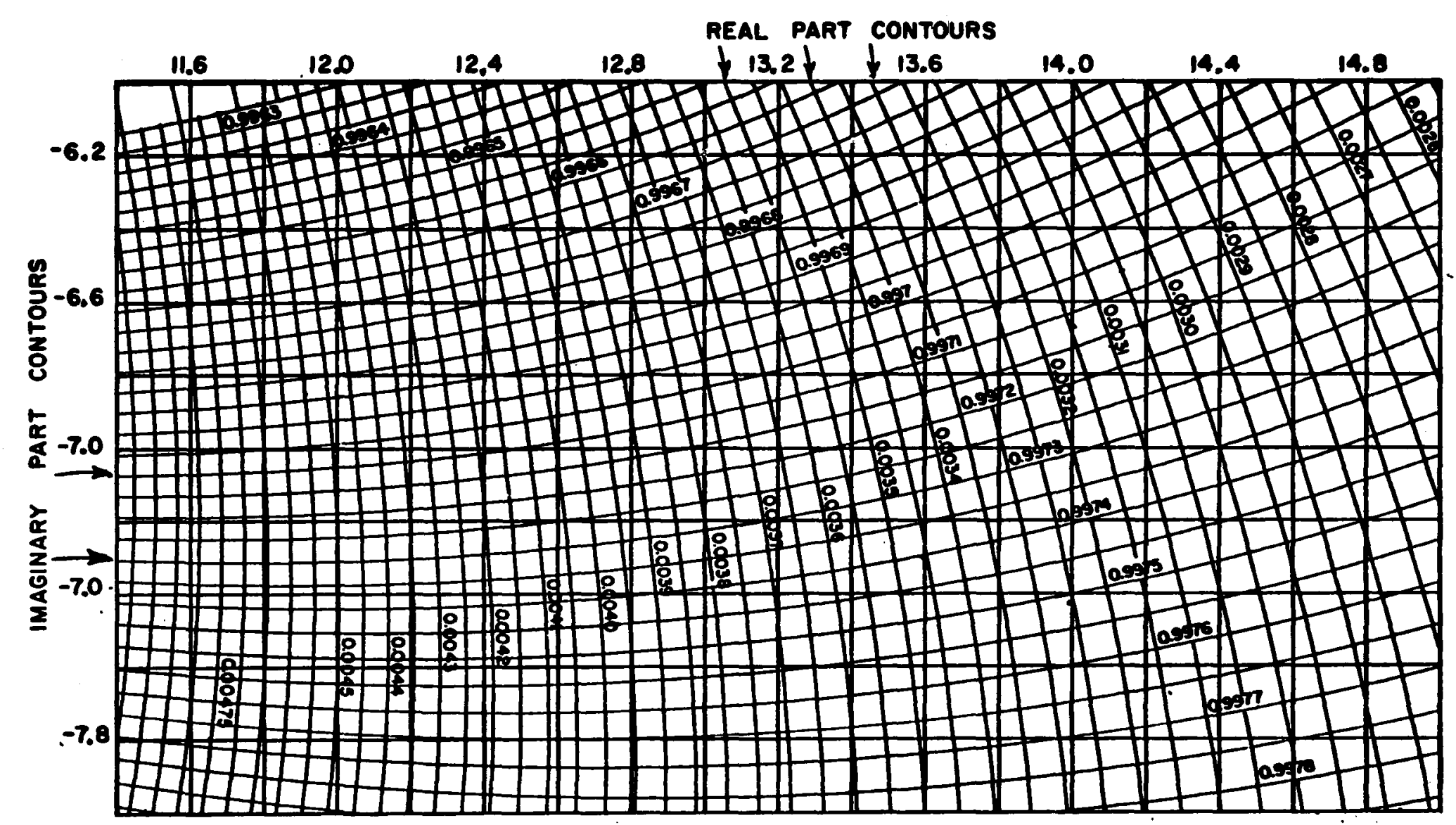

Fig. 85--Contours of $X_{1}\left(v_{r} \rho_{0}\right)$ in the region $11.4 \leq v_{r}^{\prime} p_{0} \leq 15.0$ and $-6.0 \geq v_{r}^{\prime \prime} \rho_{0} \geq-8.0$.

5 


\section{REFERENCES}

1. Stratten, J.B., Electromagnetic Theory, McGraw-Hill Book Company, Inc., New York and London, (1941).

2. Harrington, R.F., Time-Harmonic Electromagnetic Fields, McGraw-Hill Book Company, Inc., New Yor, Toronto, and London, (1961).

3. Jones, D.S., The Theory of Electromagnetism, Pergamon Press, Oxford, London, New York, and Paris, (1964).

4. Baird, R.C., "RF Measurements of the Speed of Light," Proc. IEEE, Vol. 55, No. 6. pp. 1032-1039, (June 1967).

5. Bussey, H.E., "Measurement of RF Properties of Materials; A Survey," Proc. IEEE, Vol. 55, No. 6, pp. 1046-1053, (June 1967).

6. Helberg, H. W., and Wortenberg, B., "Absorption and Transmission of Electromagnetic Waves; Phase C: Electric and Magnetic Properties of Absorber Materials in the Frequency Range from 3. $10^{9}$ to $2.10^{10} \mathrm{c} / \mathrm{s}, "$ Annual Scientific Report, Contract AF 61(052)-667, Physikalisches Institut der Universetăt Gôttingen, Sponsored by the AFSC, USAF, (1965) 
7. "A Shielded Strip Slotted Line Sample Holder for the Measurement of Complex Permittivity and Permeability at Microwave Frequencies," Tech. Rept. AFML-TR-66-315 Conductron Corporation, Ann Arbor, Michigan, (December 1966).

8. Luoma, E.J., Epstein, D.J., and Mason, S.J., "Measurement Technique for Complex Permeability and Permittivity of Highly Conductive Materials at Microwave Frequencies, " Tech. Rept. AFML-TR-66-319, Emerson and Cuming, Inc., Canton, Massachusetts, (July 1966).

9. van de Holst, H.C., Light Scattering by Small Particles, John Wiley and Sons, Inc., New York, (1957).

10. Abranowitz, M., and Stegun, I. A., Handbook of Mathematical Functions, National Bureau of Standards, U.S.A. (2nd printing, 1964).

11. Young, J.D., "Constitutive Parameter Measurements Using the Scattering Technique, "Report 1903-4, 6 February 1967, ElectroScience Laboratory, The Ohio State University Research Foundation; prepared under Contract NSR-36-008-U27 for National Aeronautics and Space Administration, Office Grants and Research Contracts, Washington, D.C.

12. Spentzos, C., "Measurements of the Constitutive Parameters from the Scattered Fields of Small Spherical Samples," Report 2382-4, 8 December 1967, ElectroScience Laboratory, 
The Ohio State University Research Foundation; prepared under Contract F33615-67-C-1507 for Air Force Avionics Laboratory, Wright-Patterson Air Force Base, Ohio. (AD 824 175)

13. Yu, J.S., "Determination of the Constitutive Parameters at Microwave Frequencies by the Scattered Fields of a Spherical Body, " Report 2148-4, 10 October 1966, ElectroScience Laboratory, The Ohio State University Research Foundation; prepared under Contract AF 33(615)-3461 for Air Force Avionics Laboratory, Wright-Patterson Air Force Base, Ohio. (AD 803 463).

14. Richmond, J.H., "Scattering by an Arbitrary Array of Parallel Wires, " Report 1522-7, 20 April 1964, ElectroScience Laboratory, The Ohio State University Research Foundation; prepared under Contract N123(953)-31663A for U.S. Nave Electronics Laboratory, San Diego, California. (AD 443 833).

15. Crout, P.D., "A Short Method for Evaluating Determinents and Solving Systems of Linear Equations with Real and Complex Coefficients," AIEE Trans., 60, pp. 1235-1241, (1941).

16. Lawrie, R.E., and L. Peters, Jr., "Modifications of Horn Antennas for Low Sidelobe Levels, "IEEE Trans. on Antennas ans Propagations, Vol. AP-14, No. 5, pp. 605-610, (September 1966). 
17. Jusik, H., Antenna Engineering Handbook, McGraw-Hill Book Company, Inc., New York, N.Y., (1961).

18. Chu, T.S. and R.A. Semplak, "Gain of Electromagnetic Horn," Bell System Technical Journal, Vol. 64, No. 3, pp. 523-537, (March 1965). 